\title{
Les espaces \\ des (im)possibles
}

Les médias en Afrique du Nord depuis les années 1990

Sous la direction de Bachir Benaziz,

Abdelfettah Benchenna et Dominique Marchetti

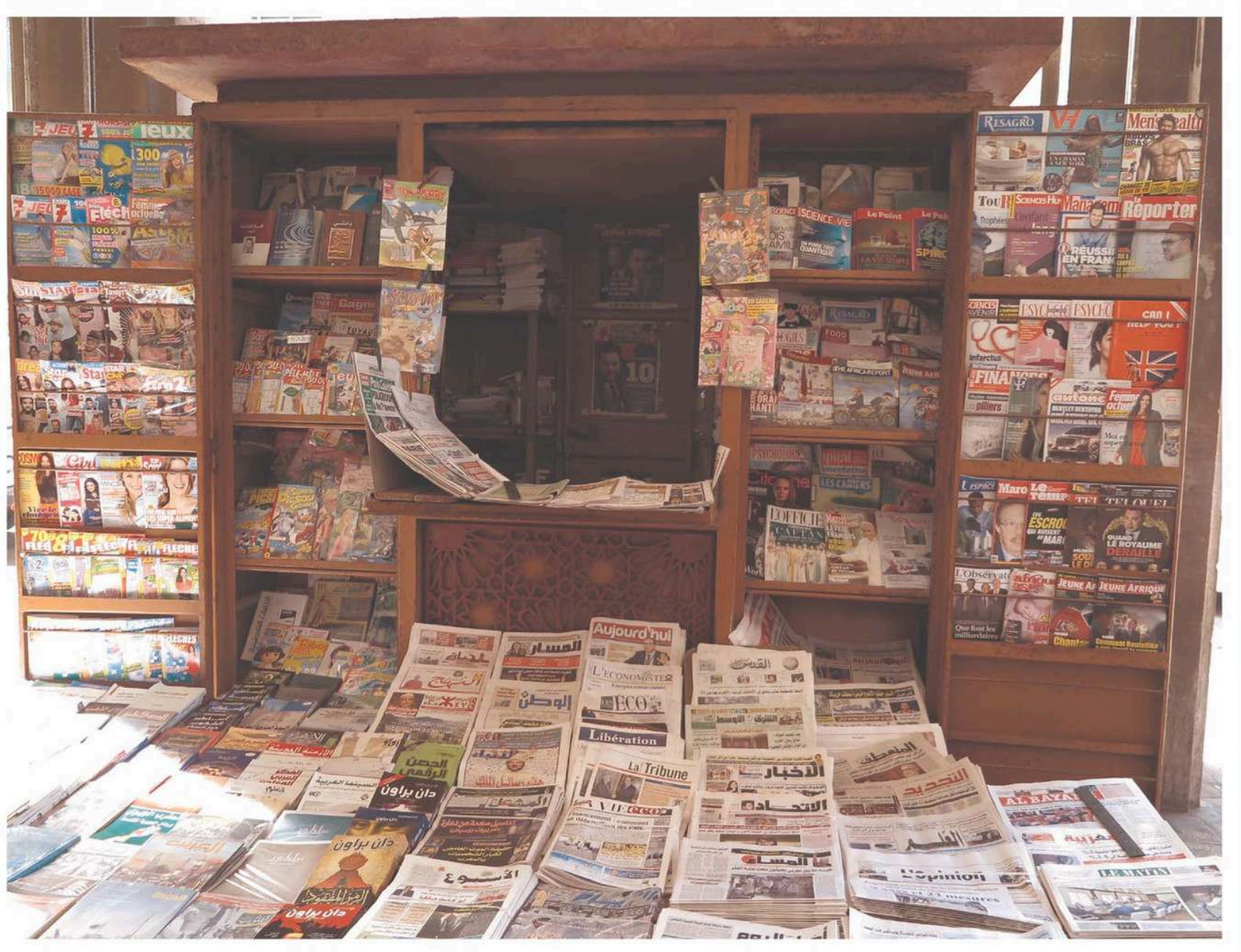




\section{Les Espaces des (im)possibles}

Les médias en Afrique du Nord depuis les années 1990

\section{Bachir Benaziz, Abdelfettah Benchenna et Dominique Marchetti (dir.)}

DOI : 10.4000/books.cjb.1952

Éditeur : Centre Jacques-Berque

Lieu d'édition : Maroc

Année d'édition : 2021

Date de mise en ligne : 1 mars 2022

Collection : Description du Maghreb

EAN électronique : 9789920347273

\section{OpenEdition}

\section{Books}

https://books.openedition.org

\section{Édition imprimée}

Date de publication : 1 janvier 2021

EAN (Édition imprimée) : 9789920347457

Nombre de pages : 244

\section{Référence électronique}

BENAZIZ, Bachir (dir.) ; BENCHENNA, Abdelfettah (dir.) ; et MARCHETTI, Dominique (dir.). Les Espaces des (im)possibles : Les médias en Afrique du Nord depuis les années 1990. Nouvelle édition [en ligne]. Rabat : Centre Jacques-Berque, 2021 (généré le 04 mars 2022). Disponible sur Internet : <http:// books.openedition.org/cjb/1952>. ISBN : 9789920347273 . DOI : https://doi.org/10.4000/books.cjb. 1952.

Légende de couverture

Un kiosque à journaux dans le centre de Rabat

Crédits de couverture

Dominique Marchetti

(c) Centre Jacques-Berque, 2021

Conditions d'utilisation:

http://www.openedition.org/6540 


\section{RÉSUMÉS}

De façon paradoxale, l'étude du journalisme et des médias occupe une place très réduite dans les recherches en sciences sociales et humaines sur l'Afrique du Nord, alors même que les espaces médiatiques dans cette région connaissent depuis les décennies 1990 et 2000 des transformations importantes. Celles-ci se rapportent notamment à l'émergence de nouveaux " acteurs » autres que l'État et les formations politiques autorisées, qui investissent désormais dans ce secteur jugé stratégique : hommes d'affaires, journalistes, communicants, spécialistes du marketing, membres d'organisations internationales, etc. Pour décrire ces bouleversements, les auteurs et autrices de l'ouvrage proposent des enquêtes de terrain en Algérie, en Égypte, au Maroc et en Tunisie, analysant conjointement les transformations des espaces médiatiques, économiques et politiques, ainsi que leurs relations.

En dépit d'une série d'invariants, les trajectoires et les histoires spécifiques de chaque espace national demeurent centrales. La lutte pour la définition des normes professionnelles, les conditions d'exercice du journalisme, la structuration des espaces médiatiques et leurs relations aux champs du pouvoir nationaux varient fortement d'un pays à l'autre. L'ouvrage pointe également la nécessité de comprendre concrètement ce qui est autorisé ou ne l'est pas selon les périodes. L'étude des nouvelles institutions de "régulation» des médias, les conditions d'émergence et de développement de chaînes de télévision, de titres de presse privés non partisans et du déploiement de "programmes d'aide aux médias " sont les trois entrées privilégiées pour saisir les délimitations de l'espace des possibles dans la production et la diffusion de l'offre d'informations.

\section{BACHIR BENAZIZ (DIR.)}

Bachir Benaziz, enseignant-chercheur contractuel à l'Université Côte d'Azur, membre de l'URE Transitions (Médias-Savoir-Territoires), ancien post-doctorant à l'ERC Tarica.

\section{ABDELFETTAH BENCHENNA (DIR.)}

Abdelfettah Benchenna, enseignant-chercheur à l'Université Sorbonne Paris Nord, membre du Laboratoire des sciences de l'information et de la communication (LabSIC).

\section{DOMINIQUE MARCHETTI (DIR.)}

Dominique Marchetti, chercheur au Centre national de la recherche scientifique (CNRS), affecté au Centre européen de sociologie et de science politique (CNRS, EHESS, Université Paris 1). 


\section{Les espaces \\ des (im)possibles}

Les médias en Afrique du Nord depuis les années 1990

Sous la direction de Bachir Benaziz,

Abdelfettah Benchenna et Dominique Marchetti

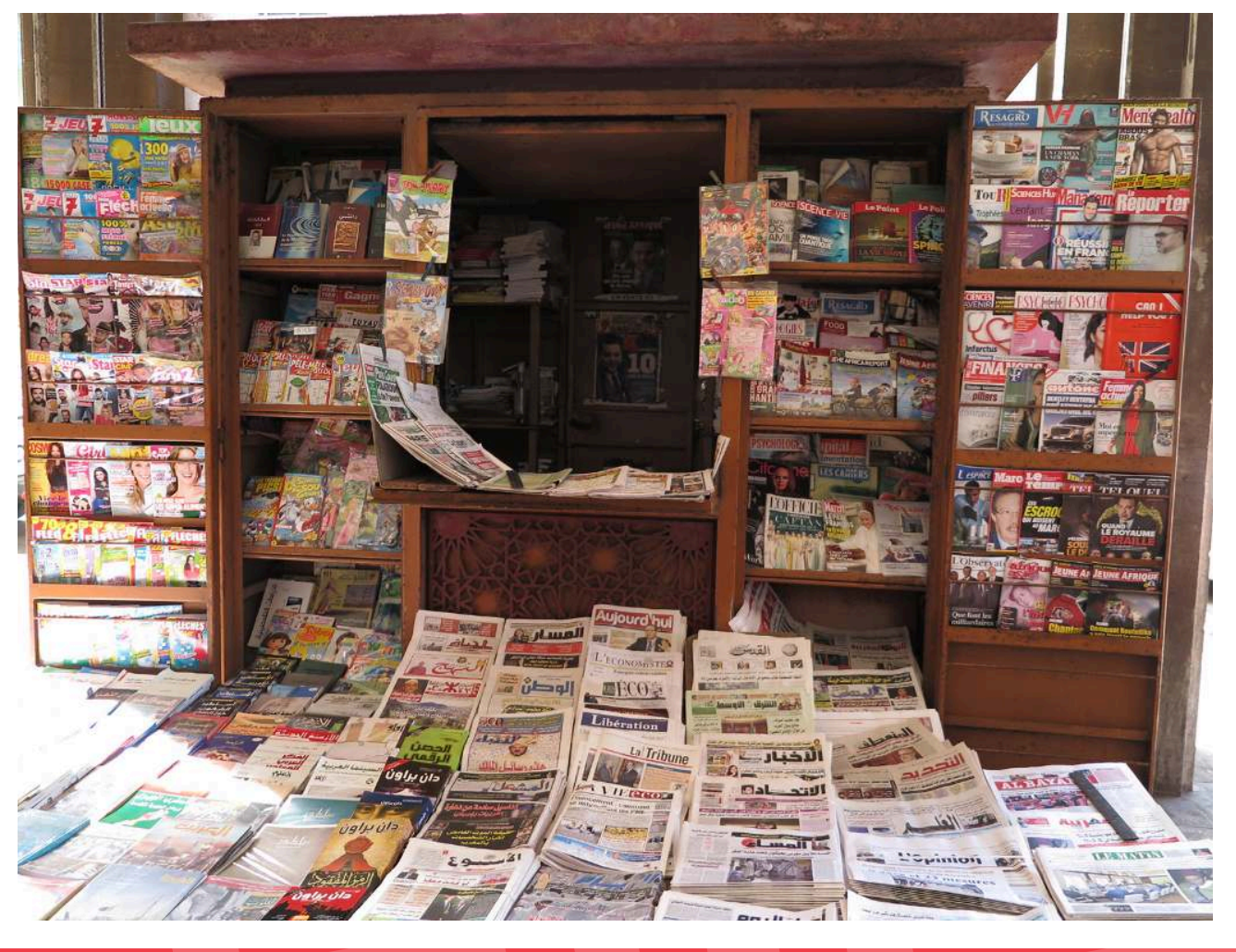

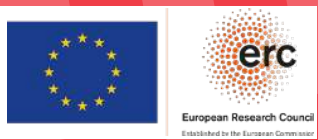

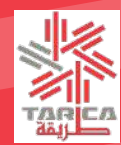

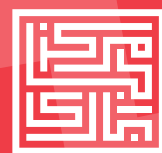

Centre Jacques-Berque

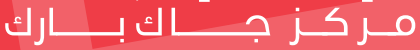
études en sciences humaines et sociales للابحاث في الهلوم الانسانية و الاجتماعية 
Les espaces des (im)possibles

Les médias en Afrique du Nord depuis les années 1990 
This publication has received funding from the European Research Council (ERC) under the European Union's Horizon 2020 Research and Innovation Programme (grant agreement $n^{\circ}$ 695674).

Cette publication a bénéficié du soutien du Conseil européen de la recherche (ERC), Programme Horizon 2020, projet ERC TARICA (convention de subvention $\left.n^{\circ} 695674\right)$.

Crédit de la photo de couverture:

Un kiosque à journaux dans le centre de Rabat: Dominique Marchetti 
Sous la direction de

Bachir Benaziz, Abdelfettah Benchenna

et Dominique Marchetti

\section{Les espaces des (im)possibles \\ Les médias en Afrique du Nord depuis les années 1990}

Centre Jacques-Berque

2021 
(c) Centre Jacques-Berque, 2021

Centre Jacques-Berque, USR3136

35, avenue Tarik Ibn Ziyad, Rabat, Maroc

www.cjb.ma

Dépôt légal : 2021MO5321

ISBN : 978-9920-34-745-7

ISBN pour la version numérique: 978-9920-34-727-3

Les espaces des (im)possibles : les médias en Afrique du Nord depuis les années 1990, sous la direction de Bachir Benaziz, Abdelfettah Benchenna et Dominique Marchetti, collection Description du Maghreb, 2021, [en ligne], http://books.openedition.org/cjb/ 


\section{Sommaire}

\section{Introduction}

Bachir Benaziz, Abdelfettah Benchenna, Dominique Marchetti .....

Partie I. Des dispositifs de «régulation » indépendants?

1. Le difficile déploiement des « instances indépendantes » dans la Tunisie en «transition » : le cas de la régulation de l'audiovisuel pendant les élections de 2019

Larbi Chouikha

2. Le Conseil national de la presse au Maroc: organe d'autorégulation de la profession de journaliste ou nouvel instrument de contrôle?

Ahmed Hidass

3. La régulation très contrôlée du champ médiatique en Algérie Chérif Dris

Partie II. Les enjeux de la reconfiguration des espaces médiatique, politique et économique

4. Une figure centrale mais éphémère du journalisme en Égypte: le journaliste du «mouvement social» Bachir Benaziz

5. L'audiovisuel en Égypte de 2000 à 2020: un secteur sous contrôle Maria Adib Doss

6. Une «libéralisation» économique, politique... et médiatique: les conditions de l'émergence de deux journaux économiques marocains à partir des années 1990 
Partie III. Les programmes français $\mathrm{d}^{\prime}$ «aide aux médias » et leurs appropriations: le cas de la Tunisie

7. La télévision étatique tunisienne, un espace d'expérimentation de la «transition médiatique» au lendemain de 2011 : le rôle-clé des acteurs français de «l'aide aux médias»

Chirine Ben Abdallah

8. Au chevet du journalisme tunisien? Fabrique de l'aide étrangère, effets sur la profession et stratégies de réappropriation

Simon Mangon

Les contributeurs 
Introduction

\section{Les trajectoires différenciées des espaces médiatiques nationaux en Afrique du Nord Le développement contrôlé des médias "privés » (Algérie, Égypte, Maroc, Tunisie) à partir des décennies 1990 et 2000}

Bachir Benaziz, Abdelfettah Benchenna, Dominique Marchetti

Une lecture des travaux les plus récents sur les médias en Afrique du Nord ${ }^{1}$ révèle l'importance et la rapidité des transformations des espaces médiatiques dans cette région du monde depuis les années 1990. Celles-ci se rapportent à une série de processus concomitants liés à des mouvements plus généraux de «libéralisation » économique et politique faisant suite à des «injonctions internationales »: l'émergence de nouveaux acteurs privés (hommes d'affaires, journalistes, experts en marketing-communication, ingénieurs-informaticiens, éditeurs, membres d'organisations «d'aide aux médias», etc.) autres que l'État et les formations politiques autorisées, qui investissent désormais dans ce secteur stratégique (par exemple, Benslimane, 2015; Belkacem, 2019; Ballarini, 2020) ; l'apparition de nouveaux modes de production journalistique diversifiant l'offre médiatique (Adib Doss, 2018; Benaziz, 2018), d'espaces alternatifs de revendications politiques et sociales avec le développement fulgurant de la presse en ligne (Ksikes, Benchenna, Marchetti, 2017; Dris, 2017) et le poids de plus en plus important des réseaux sociaux dans la production, la circulation et la réception de I'information (El Chazli, 2011; Lecomte, 2011 ; Touati, 2012; Najar, 2013; Khamis, Vaughn, 2014), non sans céder à certaines illusions politiques et technophiles comme l'ont montré certains auteurs (Mattelart et al.,

\footnotetext{
${ }^{1}$ On se reportera utilement à l'encadré à la fin de l'introduction. Voir aussi Chouikha et al., 2017.
} 
2019) ; l'extension des publics ${ }^{2}$ autrefois restreints des lecteurs des presses partisanes et officielles ou des téléspectateurs des télévisions publiques nationales. Cet ouvrage se propose donc d'apporter une série d'éclairages sur ces transformations contemporaines des espaces médiatiques en Afrique du Nord depuis les années 1990. Il est issu d'un travail collectif mené avec les autrices et auteurs dans le cadre du programme Tarica financé par le Conseil européen de la recherche ${ }^{3}$ et d'un réseau de recherche existant depuis 2015 sur les productions et circulations des biens culturels à travers le cas des pays du Moyen-Orient et de l'Afrique du Nord, soutenu par le Labex ICCA (industries culturelles et création artistique) ${ }^{4}$.

\section{Biais analytiques et conditions de production des recherches}

Les auteurs et autrices rassemblés ici et rattachés à différentes disciplines (sciences de l'information et de la communication, science politique, sociologie) proposent tout d'abord et avant tout des enquêtes de terrain sur les médias en Algérie, en Égypte, au Maroc et en Tunisie. C'est déjà un acquis au regard de l'intensification de la recherche assise, "à distance» via les réseaux sociaux et la presse, des enquêtes de courte durée à la manière des expertises spécialisées ou encore des synthèses sans enquête de "première main ». Si la priorité est ici donnée à la production de connaissances, on voudrait souligner au moins en passant quelques contraintes qui pèsent sur le travail des chercheurs et limitent leurs enquêtes. Entre la méfiance ou I'hostilité déclarée des autorités et d'une partie des enquêtés, les craintes de représailles, le soupçon de travailler pour les «services», etc., l'accès à certains terrains en Afrique du Nord est compliqué, voire dans certains cas quasi impossible, les cas algérien et surtout égyptien étant les plus fermés aux enquêtes. S'agissant de l'Égypte, la répression policière cible désormais non seulement les journalistes, mais également des universitaires et des chercheurs. Les refus d'entretien essuyés par des auteurs et des autrices

\footnotetext{
2 Par exemple, une série de travaux ont pointé l'importance des publics liés à l'immigration: Mattelart, 2007; Lecomte, 2009; Benchenna, 2014; Azizi, 2016a, 2016b.

${ }^{3}$ Dans le cadre de ce programme (https://tarica.hypotheses.org/a-proposà) et en vue de réaliser cet ouvrage, une journée d'étude s'est tenue les 4 et 5 février 2021 au Campus Condorcet à Aubervilliers. Tarica a entièrement financé la publication présentée ici. On tient ici à remercier tout particulièrement Emna Khemiri pour sa contribution à la réalisation de cet ouvrage.

${ }^{4}$ https://culturmena.hypotheses.org/
} 
de l'ouvrage, la gestion parfois délicate de leur confidentialité tout comme I'impossibilité de disposer de données statistiques élémentaires même quand elles existent sont des exemples révélateurs de ces obstacles. À cela s'ajoutent la précarité des institutions d'enseignement et recherche en Afrique du Nord et leur faible autonomie scientifique, même si la présence dans cet ouvrage de collègues (Larbi Chouikha à Tunis, Chérif Dris à Alger et Ahmed Hidass à Rabat) exerçant dans trois institutions publiques de formation au journalisme dans leur pays fait figure de contre-exemple. C'est donc tout l'intérêt des travaux présentés ici dont on ne mesure que rarement les conditions de réalisation.

En dépit de leurs limites, ces recherches essaient ensuite de rompre avec une série de biais analytiques très présents dans les travaux de sciences sociales portant sur les champs médiatiques dans des pays qui encadrent fortement la production et la diffusion de l'information. L'ampleur des transformations contemporaines consécutives au développement de médias privés non partisans dans les années 1990 et/ou 2000 invite en premier lieu à écarter toute démarche politico-centrée et réductionniste, selon laquelle «les États» ou «les appareils» ou «les régimes» seraient tout puissants et imposeraient par "le haut» leur volonté. Les limites de ces études, souvent empreintes de culturalisme et fascinées par les microcosmes des pouvoirs, ont été suffisamment démontrées pour ne pas s'y arrêter. Les reconfigurations des espaces médiatiques au Maroc comme en Algérie, en Tunisie et en Égypte ne peuvent en effet être étudiées uniquement à l'aune des «ouvertures» / «fermetures» politiques et institutionnelles accordées ou imposées par les régimes en place, ces périodes étant souvent délimitées par des bornes historiques précises au détriment d'une analyse processuelle. L'importance des contraintes politiques qui pèsent sur les médias dans ces pays a amené les observateurs à centrer longtemps l'attention sur les seuls agents et institutions étatiques, ainsi que sur l'évolution des régimes juridiques de la presse. Malgré leur intérêt indéniable, la plupart de ces travaux souffrent d'un biais institutionnel, oubliant non seulement les trajectoires des journalistes et des entreprises médiatiques dans toutes leurs dimensions, mais aussi le poids des logiques transnationales.

En deuxième lieu, comme pour un certain nombre de pays classés comme "autoritaires» ou appartenant aux zones géopolitiques ou géolinguistiques où ils se concentreraient (l'expression culturaliste "autoritarisme arabe» en est un exemple caricatural), les quatre pays concernés par cette étude font l'objet d'une surveillance internationale. Les experts des organisations internationales spécialisées comme certains 
chercheurs entendent explicitement ou implicitement mesurer le degré supposé de "démocratisation" de leurs champs médiatiques et son évolution (Naimi, 2016). Si ce biais évolutionniste est logique dans le cadre des luttes politiques, il ne l'est pas dans le champ scientifique. Il est le produit de la concurrence au sein de certaines fractions des champs académiques nationaux et transnationaux, tout particulièrement en science politique et/ou dans les études dites «d'aires culturelles». Ses luttes normatives et académiques se jouent notamment à travers les appellations données aux espaces politiques et/ou aux univers médiatiques des pays d'Afrique du Nord (" autoritaire», «hybride», etc.) ou caractérisant le fonctionnement du champ du pouvoir («ambivalent authoritarianism», "competitive authoritarianism», etc.), alors même que c'est précisément un enjeu de lutte politique qu'il faut prendre pour objet. C'est d'autant plus vrai que la pensée typologique «qui découpe des populations en classes identifiées» (Bourdieu, Saint-Martin, 1978, p. 4), ou ici en l'occurrence des pays, en vient souvent explicitement ou implicitement à opposer, d'une part, la propriété étatique des médias qui signifierait leur contrôle ou leur forte dépendance et, d'autre part, la propriété privée qui serait synonyme de «liberté » politique et/ou commerciale. Les auteurs d'un livre récent (Richter, Kozman, 2020, p. XXXI) consacré aux médias dans «l'espace arabe » ont bien pointé les limites de cette vision typologique, qui sont confirmées par les résultats de recherche présentés ici.

En troisième lieu, ce travail permet de décaler le regard par rapport aux études sur les médias et plus largement les productions culturelles, qui ne voient ces univers qu'à travers des «lunettes» commerciales et économiques. Pourtant, poser «la maximisation du profit en clef de compréhension unique de la rationalité économique» (Duval, Garcia, 2012, p. 13) est une erreur dans des espaces où le symbolique et/ou le politique constituent également des logiques centrales. Comme le montrent certaines contributions de ce livre, les luttes autour de ce que signifie «l'indépendance» des médias ou de leurs propriétaires réels ou supposés et les formes de discrédit récurrent viennent rappeler que les enjeux au sein des espaces médiatiques sont au moins sinon plus politiques et professionnels qu'économiques. C'est d'autant plus vrai qu'une fraction non négligeable des entreprises de presse dans les pays d'Afrique du Nord sont, comme dans le champ de l'édition française par exemple (Noël, 2021), des petites structures qui ont du mal à vivre et sont dans une perspective de désintéressement à l'opposé de la vision commerciale. La grande insécurité sociale et économique de ce secteur, du 
fait des transformations très rapides des modèles économiques (Powers, Russel, 2020, p. 10), y est particulièrement exacerbée.

C'est surtout d'autant plus fort que l'espace du dicible dans les champs politiques et médiatiques des pays de cette zone géographique est borné par une multiplicité de «lignes rouges» en lien avec le politique, le religieux, l'armée et l'intégrité territoriale. Comme le montrent par exemple Chérif Dris, Ahmed Hidass, Maria Adib Doss dans leurs chapitres respectifs sur l'Algérie, le Maroc et l'Égypte, toute critique ou remise en cause de l'une de ces «lignes rouges» est passible de sanctions judiciaires et est considérée comme une "traîtrise » à l'égard de la nation, d'où ce rappel fréquent au «patriotisme» des membres des autorités quand ils s'adressent aux journalistes considérés comme critiques. De même, le poids de I'histoire nationale et ses effets sur les productions journalistiques ont été bien documentés à propos du Maroc (Saleh, 1995; Zaid, 2017; Benchenna, Ksikes, Marchetti, 2017; Benchenna, Marchetti 2019; El Issawi, 2021, p. 932-934), notamment l'importance de l'autocensure (El Kadoussi, 2020).

\section{Les espaces des (im)possibles}

\section{Des dispositifs de « régulation » indépendants?}

L'ouvrage se propose donc d'étudier ces transformations des espaces médiatiques nationaux en Algérie, en Égypte, au Maroc et en Tunisie à travers trois entrées qui se veulent complémentaires. La première porte sur les instances dites de "régulation», fondées à la suite des «mouvements révolutionnaires» de 2011. Larbi Chouikha, Chérif Dris et Ahmed Hidass décrivent la genèse de ces instances, les types de groupe qui les composent et les modalités de leur sélection, leurs fonctions (réelles ou affichées) et les contraintes qui s'opposent à l'accomplissement de leurs missions. Autrement dit, ils restituent leurs rapports au pouvoir politique et aux différents agents qui interviennent dans le champ médiatique. Plus largement, ils s'interrogent sur l'adéquation ou non de ces instances de «régulation » ou de «contrôle» avec les réalités actuelles des espaces médiatiques des pays étudiés.

Dans le premier chapitre, consacré à la régulation audiovisuelle dans la Tunisie post-Ben Ali, Larbi Chouikha questionne ainsi les marges de manœuvre de ce type d'instance: d'une part, dans une configuration politique particulièrement instable; d'autre part, dans un paysage 
médiatique en pleine effervescence, marqué notamment par l'émergence d'hommes d'affaires à la tête de chaînes de télévision commerciales et participant à la lutte pour la conquête du pouvoir. À travers l'analyse des activités de la Haute instance pour la communication audiovisuelle (HAICA) durant les élections de 2019 et en s'appuyant sur des entretiens avec les acteurs concernés, son travail montre les limites et les paradoxes de cette instance. Censée garantir, entre autres, «le pluralisme des idées», "la neutralité des médias» et "l'égalité des chances entre tous les candidats», la HAICA reste impuissante, ne pouvant faire respecter ou appliquer ses décisions, du fait notamment de la fragilité des institutions de l'État et de l'équilibre politique qui s'est instauré après le départ de Zinedine el-Abidine Ben Ali. Son existence reste toutefois nécessaire, au regard des "standards internationaux en matière de régulation démocratique», et inséparable des stratégies de légitimation politique des différents gouvernements post-2011.

À l'inverse, dans le chapitre 2, qui porte sur le Conseil national de la presse créé en 2018 au Maroc (CNP), Ahmed Hidass s'interroge sur la nature même et les objectifs réels de ce nouvel organe chargé d'administrer la profession journalistique: s'agit-il d'une véritable instance d'auto-régulation du journalisme et de promotion de la liberté de la presse ou d'un instrument de contrôle et de censure au service du pouvoir? À partir de l'étude de la genèse et des premières réalisations du CNP, l'auteur pointe dans ce texte les contradictions constitutives de cette instance. «Hybride» à la fois dans sa composition, ses compétences et son mode de financement, le CNP tranche tout d'abord avec les différentes traditions et modèles nationaux de régulation de la profession de journaliste. C'est le cas notamment de son code de déontologie, dont le caractère obligatoire s'oppose à « la valeur usuelle des codes d'éthique journalistique dans le monde». Financé par l'État mais administré par des professionnels du métier, héritier d'une approche restrictive de la liberté d'expression et ne concernant que la presse privée, fondé dans un contexte global de durcissement et de diversification des formes de répression à l'égard des journalistes au Maroc, le CNP semble cumuler tous les attributs d'un instrument de contrôle. En témoignent ses premières sorties médiatiques et rapports qui sont en contradiction et/ou en décalage avec les préoccupations des professionnels du secteur et des réalités actuelles de l'espace médiatique marocain.

Dans le chapitre 3, Chérif Dris pose plus globalement la problématique de la «gestion de la régulation » des médias algériens dans leur diversité, dans un contexte marqué par l'emprise du champ politique sur l'espace médiatique et une série de transformations importantes liées au développement 
des chaînes de télévision privées et des sites d'information en ligne. En s'appuyant sur un important travail documentaire et des entretiens, l'auteur montre comment la question de la «régulation» ne peut être dissociée des conditions politiques et économiques qui garantissent, ou non, l'autonomie et l'efficacité des instances chargées de veiller au «bon fonctionnement» de ce secteur. Dans un premier temps, Chérif Dris analyse le dispositif juridique et législatif mis en place à partir de 2012 afin de «mettre de l'ordre» dans un marché médiatique dérégulé à la suite des politiques de «libéralisation » relative des années 1990 et 2000 . II insiste sur le caractère très politique et très contraignant de ces textes, notamment en ce qui concerne la presse en ligne, souvent plus critique à l'égard du pouvoir. Dans un second temps, l'auteur revient, d'un côté, sur les différents obstacles qui entravent la mise en œuvre des mécanismes de régulation consacrés par les textes, de l'autre, sur le décalage existant entre ces derniers et les réalités du terrain. Le cas de l'autorité de régulation audiovisuelle (ARAV), créée en 2014, est particulièrement révélateur des dysfonctionnements et contradictions qui relativisent fortement la portée des missions assignées à ce type d'instance. Fortement dépendante à l'égard du pouvoir politique, notamment à travers le système de nomination, l'ARAV est en effet censée "encadrer un champ audiovisuel composé essentiellement de chaînes privées dont l'existence légale n'est pas reconnue».

\section{Les enjeux de la reconfiguration des espaces médiatique, politique et économique}

La deuxième partie porte sur les enjeux de la restructuration des espaces médiatiques nationaux en Afrique du Nord à la suite du développement des médias privés. Ceux-ci sont appréhendés à partir de deux focales sur l'Égypte des années 2000 à aujourd'hui - l'une sur la presse écrite et l'autre sur la télévision - et une dernière sur le Maroc à partir de la décennie 1990. Au travers d'enquêtes de terrain inédites, notamment par entretiens, les trois chapitres décrivent les conditions historiques de possibilité de l'arrivée des médias privés. Ils pointent surtout, chacun à leur manière, les limites à géométrie variable selon les périodes historiques de l'espace des (im)possibles journalistiques, qui est le produit de la relation entre univers politique, économique et médiatique. En effet, loin des analyses des économistes libéraux, qui considèrent que «l'ouverture au secteur privé» des marchés médiatiques serait synonyme d'une plus grande «liberté » à l'égard des univers politiques, les auteurs montrent comment ces processus 
sont très encadrés par les fractions dominantes du champ du pouvoir national, tout en n'excluant pas ici et là des marges de manœuvre.

Ainsi, dans le chapitre 4 tiré de sa recherche doctorale (Benaziz, 2018), Bachir Benaziz restitue ce qu'il appelle "une parenthèse notoire dans I'histoire de la presse écrite en Égypte» dans les années 2000, c'est-à-dire sous le régime de Hosni Moubarak. Elle est la conjonction du développement des mouvements sociaux, du succès des premiers journaux privés non partisans et de l'arrivée d'une nouvelle génération de journalistes. Il analyse la légitimation de figures de "journalistes-justiciers» qui se spécialisent dans le traitement de ces protestations, en valorisant le «reportage» et le «terrain ». Bachir Benaziz nous fait tout particulièrement entrer dans les logiques de production du quotidien Al-Masry Al-Youm (L'Égyptien aujourd'hui), fondé en 2004 par l'homme d'affaires Salah Diab et l'éditeur Hisham Kassem. Il décrit comment ses dirigeants promeuvent de nouvelles "actualités», qui non seulement ne sont pas uniquement centrées sur le Caire, mais sont aussi produites avec des sources d'informations alternatives aux sources officielles, notamment les chercheurs et les militants syndicaux. La constitution de ces nouvelles positions dans l'univers journalistique égyptien doit beaucoup aux trajectoires de leurs promoteurs, «issus pour la plupart de catégories sociales relativement défavorisées», notamment celle des «fonctionnaires» paupérisés durant l'ère Moubarak (1981-2011). II retrace ainsi les parcours professionnels emblématiques de deux anciens journalistes d'Al-Dustûr (La Constitution) et d'Al-Badîl (L'alternative), Mostapha Bassiouny, rendu célèbre pour sa couverture des protestations sociales des années 2000, et Khaled El-Balchy, figure emblématique de la presse égyptienne. Au-delà du fait qu'ils incarnent cette nouvelle figure du «journaliste justicier » pratiquant ainsi une forme de "revanche sociale», ils montrent également comment ils se heurtent à partir de 2013 aux nouvelles formes de contrôle imposées par le retour des militaires au pouvoir.

Dans le chapitre 5, Maria Adib Doss apporte également une analyse de "l'ouverture» au secteur privé de l'espace médiatique égyptien, cette foisci à partir de l'arrivée des premières chaînes de télévision commerciales au début des années 2000, puis de leur "confiscation», pour reprendre son expression, lors du retour des dirigeants militaires à partir de 2013. En effet, après 2001, année de la création de la première chaîne satellitaire privée nationale, Dream TV, fondée par un homme d'affaires égyptien, d'autres vont suivre rompant le monopole de l'État sur l'information. S'appuyant sur une enquête réalisée dans le cadre de sa thèse entre 2013 et 2018 (Adib Doss, 2018) et complétée par des entretiens menés en 2020, l'autrice propose de 
cerner comparativement les modalités du contrôle de ces chaînes à deux époques: la fin du règne de Hosni Moubarak dans les années 2000 et le début de celui d'Abdel Fattah Al-Sissi depuis 2013. Elle s'appuie sur trois terrains: la régulation formelle et institutionnelle, les modes de propriété et la régulation informelle.

S'agissant du premier d'entre eux, Maria Adib Doss montre qu'en l'absence de textes jusqu'en 2016 ces chaînes étaient étroitement contrôlées via les règles régissant la «zone franche» appelée la Cité des médias dans laquelle elles étaient installées. L'autrice s'intéresse également à la manière dont, à partir de 2018, le pouvoir égyptien encadre leurs activités à travers la constitution de différentes instances de régulation, dont le Conseil suprême de la régulation des médias qu'elle étudie. La propriété des chaînes de télévision privées se caractérise durant la fin des mandats de Hosni Moubarak (du début des années 2000 jusqu'en 2011) par l'investissement d'hommes d'affaires qui obtiennent des «privilèges » en échange d'un fort contrôle. Alors que, dans les années suivant les soulèvements de 2011, les chaînes de télévision privées sont dirigées par des hommes affaires proches des différents appareils sécuritaires de l'État, ce contrôle se fait plus direct à partir de 2016. En effet, l'espace des télévisions privées égyptiennes est en 2021 la propriété d'une seule société privée, Eagle Capital, détenue par les services de renseignements. Enfin, Maria Adib Doss décrit une forme de continuité dans les modes de régulation informelle, insistant sur les délimitations à géométrie variable des "lignes rouges», les activités discrètes mais très dures des membres des services de renseignement à l'encontre des journalistes.

Le chapitre 6 apporte un troisième éclairage sur la reconfiguration conjointe des espaces médiatique, politique et économique en revenant sur les conditions du développement d'entreprises de presse privées non partisanes, dans un autre espace national, le Maroc, et à partir de la décennie précédente, celle de 1990. Les trajectoires de deux sociétés (Éco-Médias et Impression presse édition), qui éditent notamment deux titres économiques francophones L'Économiste (depuis 1991) et La Nouvelle Tribune (depuis 1995), constituent une rupture dans un espace journalistique qui jusque-là réfractait stricto sensu l'univers politique à quelques rares exceptions comme Lamalif (1966-1988) et Kalima (1986-1989) notamment. En effet, celui-ci se divisait entre la presse "officielle» et les quotidiens des partis politiques. Ces expériences s'inscrivent dans un processus de «libéralisation» économique et politique du Maroc à partir de la fin des années 1980 et du début des années 1990 pour répondre aux injonctions 
internationales de «libéralisation » des économies et des échanges. Elles sont rendues possibles par des fractions dominantes du champ du pouvoir, qui accompagnent ces deux projets économiques, politiques et journalistiques d'origine familiale, deux couples en étant à l'origine. Défendant tout à la fois la "libéralisation», la "modernisation » économique et politique du Maroc, les institutions, "les valeurs» et l'intérêt de la nation ainsi qu'une information plus "objective», leurs promoteurs développent des entreprises commerciales rentables et revendiquent ces logiques commerciales. Ils s'appuient essentiellement sur des recettes publicitaires importantes et un lectorat composé des fractions urbaines de l'espace social les plus dotées en capital économique et culturel. À partir d'éléments d'une enquête par entretien menée avec Abdelfettah Benchenna et Driss Ksikes qui a été complétée par des analyses documentaires, Dominique Marchetti s'efforce de décrire les trajectoires des principaux dirigeants de ces deux entreprises de presse, proposant ainsi une analyse du fonctionnement de l'univers de la presse économique marocaine dans ses relations aux fractions dominantes du champ du pouvoir qui délimite ses possibles.

\section{Les programmes français d'« aide aux médias " et leurs appropriations: le cas de la Tunisie}

La troisième partie de l'ouvrage décale le regard vers les dimensions transnationales de ces restructurations à travers l'exemple emblématique de la Tunisie considérée comme le «bon élève » de la région. Deux chapitres s'interrogent sur la production des programmes d'aide aux médias français et leurs appropriations par les journalistes. Tout d'abord, Chirine Benabdallah revient, dans le chapitre 7, sur un moment particulier de la reconfiguration de l'espace télévisuel tunisien, celui de la fin du régime de Zine el-Abidine Ben Ali. En observant in situ la fabrication du journal télévisé (JT) de la chaîne nationale Watanya 1, elle décrit comment sa forme et son contenu ont été transformés. La hiérarchisation des informations post-14 janvier n'est plus imposée par les dirigeants de la chaîne, qui devaient auparavant exécuter les ordres du ministère de I'Intérieur, mais elle est «dictée par l'importance des nouvelles». L'auteure relève cependant les obstacles auxquels a été confrontée l'équipe du nouveau JT. Elle montre le passage d'un «média d'État» à un «média de service public » à travers la cristallisation d'un conflit entre les responsables de la télévision publique et ceux des différents gouvernements dits "de transition», qui n'ont pas réussi «à se défaire de la volonté de contrôler les médias». 
L'intérêt majeur de ce travail empirique est qu'il apporte des connaissances nouvelles sur les débuts de l'intervention de Canal France International (CFI) et de France 24 auprès des autorités tunisiennes pour accompagner le processus de la «transition médiatique» au lendemain de la «révolution» du 14 janvier 2011. L'auteure rappelle, à juste titre, que cette intervention a été effectuée dans un contexte de "crise» entre les deux pays, suite aux déclarations de Michèle Alliot-Marie, la ministre française des Affaires étrangères, qui proposait «le savoir-faire français» à la police tunisienne pour faire face aux manifestations. Chirine Benabdallah s'intéresse à un autre domaine de l'exportation de «l'expertise française » à partir des programmes d'aide aux médias. L'autrice explique que la Tunisie de l'après-14 janvier 2011 était le premier terrain d'action de l'Académie France 24, créée pour l'occasion, et qui deviendra quelques années plus tard l'Académie France Médias Monde. Cette contextualisation permet de voir comment les «demandes » émanant de plusieurs pays en pleine restructuration politique ont constitué une opportunité pour créer l'Académie et tester en Tunisie son modèle financier et pédagogique. Le travail de Chirine Benabdallah donne à voir les interrogations que suscitent ces dispositifs de formation qui font appel notamment à des formateurs tunisiens, anciens collègues des bénéficiaires de ces formations. En effet, ces derniers s'interrogent parfois sur l'intérêt de ces sessions de formation, sur la légitimité et les compétences professionnelles de leurs anciens collègues à dispenser des sessions de formations. L'autrice met surtout l'accent sur la manière dont la reconfiguration de l'espace politique se traduit dans l'univers médiatique, insistant plus particulièrement sur les «nouveaux besoins» en formation de journalistes et les nouvelles pratiques de travail au sein de la télévision nationale.

De son côté, Simon Mangon apporte, dans le chapitre 8, un autre éclairage encore plus contemporain sur la production et la diffusion de l'aide internationale aux médias en Tunisie postrévolutionnaire. II propose une étude de cas sur le média en ligne Inkyfada et le rôle de CFI dans son accompagnement. En inscrivant son travail à l'intersection de la sociologie des médias et de la sociologie des politiques de développement, Simon Mangon réfute le mythe d'une supposée influence étrangère sur la ligne éditoriale du média. II montre plutôt que les relations avec des organisations extérieures au champ journalistique contribuent à redéfinir la vision du «bon journalisme» en Tunisie, contribuant à transformer les pratiques professionnelles et les conceptions même du métier de journaliste. Pour cela, l'auteur analyse finement la manière dont les journalistes du média 
Inkyfada se réapproprient des préoccupations des bailleurs selon leurs besoins, conservant ainsi une forme d'autonomie. Cette réappropriation se traduit tant dans la forme des productions médiatiques (mobilisation de l'infographie, du datajournalisme, photos de grande qualité, etc.), que dans les thématiques abordées, qui sont inscrites dans les cahiers des charges des bailleurs de fonds internationaux (droits humains, situation des minorités, égalité des sexes ou écologie) ou encore dans la revendication d'un «journalisme d'investigation».

En analysant la trajectoire d'Inkyfada, qui trouve son origine dans la plateforme d'un collectif de blogueurs cyberactivistes Nawat, Simon Mangon détaille quelques enjeux de la reconfiguration de l'espace médiatique tunisien : la professionnalisation des journalistes, leur positionnement éditorial, les langues mobilisées, les publics visés et enfin la place encore fragile et marginale de ce média. L'auteur dresse également une cartographie de l'espace du «développement médias» en Tunisie. Ce dernier est composé de différents types d'acteurs (bailleurs de fonds, opérateurs de mise en œuvre, partenaires locaux) de diverses nationalités (États-Unis, France, Allemagne, Belgique ou États scandinaves) qui interviennent essentiellement dans le domaine de la formation au journalisme. L'objectif central est de promouvoir les «bonnes pratiques» et de diffuser un modèle de journalisme «professionnel» défini selon leurs critères. L'auteur rappelle que ce type d'intervention n'est pas nouveau, analysant les contenus de formation dispensés par le Centre africain de perfectionnement des journalistes et des communicateurs (CAPJC) pendant quinze années (2000-2015). À partir d'une analyse comparative de quatre structures (Canal France International, Deutsche Welle Akademie, Fondation Friedrich Naumann et International Media Support), Simon Mangon insiste sur les spécificités de structure du «développement médias» qui adopte une stratégie propre en fonction de son statut, de ses bailleurs de fonds, de ses priorités, de son histoire dans le pays d'intervention et de ses partenaires locaux.

Il consacre une partie de son chapitre au secteur français de «l'aide aux médias » et donc à CFI. II apporte des connaissances nouvelles et prolonge les travaux de Chirine Benabdallah, développés dans le chapitre précédent, puisqu'il met tout particulièrement l'accent sur les logiques de concurrence, les rapports de force des acteurs en présence dont dépend CFI et des visions différentes de ce que devrait être le journalisme.

Cependant, le principal intérêt de ce chapitre réside ailleurs. L'auteur montre surtout que les instruments de "développement médias» 
contribuent paradoxalement à éloigner les journalistes du travail éditorial censé représenter leur principale activité. En effet, le travail empirique qu'il a mené au sein du journal Inkyfada en observant les activités quotidiennes de ses membres et en analysant leurs trajectoires biographiques donne à voir un média vivant au rythme des «projets » proposés par les bailleurs internationaux. Les journalistes adoptent alors les codes linguistiques propres au secteur du développement et réalisent les tâches caractéristiques de l'ingénierie des dispositifs internationaux. Considéré comme un partenaire tunisien de référence par de nombreux bailleurs étrangers, Inkyfada est devenu une sorte d'opérateur local auquel on délègue des tâches qui ne sont en général pas attribuées à un média spécifique. L'auteur explique cependant que les relations d'Inkyfada à l'univers du développement ne va pas contribuer pour autant à reproduire un «modèle» journalistique défini à l'étranger et donc à l'uniformisation des pratiques professionnelles selon des normes venues d'ailleurs. Selon lui, les journalistes cherchent à réorienter l'appui étranger selon leurs propres intérêts: ils négocient leur autonomie au quotidien au contact des bailleurs de fonds, qu'il s'agisse du modèle économique, de la ligne éditoriale ou des projets de développement qu'ils mènent.

Au final, cette analyse des reconfigurations des espaces médiatiques en Afrique du Nord depuis les décennies 1990 et 2000 vient rappeler qu'en dépit d'une série d'invariants les trajectoires et les histoires spécifiques de chaque espace national demeurent centrales dans l'analyse. La lutte pour la définition des normes professionnelles, les conditions d'exercice du journalisme, la structuration des espaces médiatiques et leurs relations aux champs du pouvoir nationaux varient fortement d'un espace national à l'autre, chacun réfractant différemment les transformations économiques, politiques et sociales transnationales.

Les contributions réunies dans cet ouvrage viennent également pointer la nécessité de comprendre l'espace des possibles et ses transformations dans ces univers médiatiques où les contraintes politiques directes ou indirectes pèsent lourdement. Rester dans son pays et continuer à y exercer les métiers du journalisme demande une connaissance fine des règles du jeu et de savoir en jouer. Pour reprendre le vocabulaire d'Albert Hirschman, la "loyalty», notamment le respect des "lignes rouges» nationales, est une condition indispensable pour s'inscrire dans la durée, la « voice» est une position difficile à tenir, ce qui explique «l'exit» fréquent (quand il est possible) vers d'autres activités professionnelles et/ou l'exil à l'étranger (Hirschman, 1970). 


\section{Une bibliographie encore sommaire}

Cet ouvrage vise à contribuer, après d'autres, à combler une lacune. En effet, l'étude du journalisme et des médias occupe une place très réduite dans les études en sciences humaines et sociales en langue française sur l'Afrique du Nord. La presse papier et électronique comme les médias audiovisuels sont souvent plus utilisés comme des sources d'information que des terrains à explorer, tout se passant comme s'ils n'étaient pas dignes d'intérêt. De même, les recherches sur les médias, tout particulièrement en France, accordent peu d'intérêt aux enquêtes dans les pays d'Afrique du Nord. Cependant, les apprentis-chercheurs et les chercheurs peuvent désormais s'appuyer sur une série de travaux dispersés sur les espaces médiatiques dans cette zone. Si on ne peut bien évidemment pas en rendre compte ici, les entreprises collectives comparables à la nôtre doivent être rappelées, notamment pour donner aux lecteurs une série de repères bibliographiques. Comme pour les recherches en sciences sociales des médias en France, ces travaux ont été longtemps dominés par des perspectives historiques et juridiques. En effet, la presse écrite (Muddathir, 1966; Souriau-Hoebrechts, 1975) ou les médias audiovisuels (Pigé, 1966) à l'échelle du Maghreb ou dans les différents espaces nationaux qui le composent ${ }^{5}$ ont fait l'objet de nombreuses enquêtes remontant à la période coloniale. De même, les recherches sur les régimes juridiques de la presse (par exemple Mollard, 1963; Abdel Rahman, 1995) sont très utiles pour comprendre les transformations du journalisme avant et après les indépendances.

À partir de la fin de la décennie 1980, des recherches complémentaires, notamment en information-communication et à un degré moindre en science politique, se sont développées entre la France, les pays francophones et ceux du Maghreb, la création de revues spécialisées en France comme en Afrique du Nord l'atteste. Cependant, au-delà de recherches individuelles pionnières sur les médias ${ }^{6}$ et d'une littérature de témoignage ${ }^{7}$ très utile, les entreprises collectives demeurent très rares. Wolfgang Slim Freund a réuni une série de chercheurs sur le thème de "l'information au Maghreb» (Freund, 1992). De même, parmi une vingtaine de collègues qui ont participé à des publications sur les «recompositions du champ médiatique dans l'Orient arabe» (Bras et

\footnotetext{
${ }^{5}$ Par exemple, pour le Maroc, on se reportera utilement à Aouchar, 1990; Baida, 1996; Berrada, 2003; Miège, 1954.

${ }^{6}$ La liste n'est bien évidemment pas exhaustive. Sur la Tunisie: Chouikha, 1992 et 1995 ; Jendoubi, 1988. Sur le Maroc: Hidass, 1983, 1988. Sur l'Algérie: Chevaldonné, 1981 ; Mostefaoui, 1989, 1992 et 1995. Sur le Maghreb et/ou le Machrek: Kraemer, 2001 ; Tiers, Ruf, 1975. Sur l'Égypte: Munir, 1990; El Khawaga, 2003; Guaaybess, 2005.

7 Pour ne citer que le cas du Maroc, on peut se reporter utilement par exemple aux travaux suivants : Daoud, 2007; Alaoui, 2012; Bensmain, 2015.
} 
Chouikha, 2002 et Mermier, 2003), quelques-uns traitent de pays d'Afrique du Nord, qui est un des parents pauvres de l'étude des médias dans la zone à laquelle elle est souvent rattachée: les pays majoritairement de langue arabe et/ou de confession musulmane.

Deux livres collectifs parus chez Actes Sud la même année (Mohsen-Finan, 2009; Gonzalez-Quijano et Guaaybess, 2009) font exception. Le premier rassemble de courts chapitres où se mêlent chercheurs, experts, essayistes et journalistes pour décrire la situation des médias dans les «mondes arabes et musulmans». On y trouve quelques textes consacrés à l'Algérie (Djafaar Saïd), au Maroc (Mohamed El Ayadi et Driss Ksikes) et à la Tunisie (Larbi Chouikha). Le second apporte également quelques contributions sur le journalisme au Maghreb, tout particulièrement sur les nouveaux médias en Algérie (Belkacem Mostefaoui), ainsi que sur la télévision en Tunisie (Riadh Ferjani). Enfin, plus récemment, Enrique Klaus et Olivier Koch ont tiré un livre d'un colloque qui s'est tenu en 2015 sur les rapports entre univers politique et médiatique dans la Tunisie post-Ben Ali (Klaus et Koch, 2020).

Plusieurs revues dites «d'aires culturelles» ont également publié des ensembles thématiques sur les espaces médiatiques en Afrique du Nord. François Chevaldonné, un des pionniers sur ces objets, a coordonné à la fin des années 1980 un numéro de la Revue des mondes musulmans et de la Méditerranée consacré aux médias audiovisuels dans le monde arabe, dont une partie porte sur l'Afrique du Nord, essentiellement sur l'Algérie (Chevaldonné, 1988). La revue $N A Q D$ a été également une des pionnières en la matière, publiant un premier numéro en 1995 où on peut trouver des recherches sur l'Algérie, l'Égypte, la Tunisie et un autre en 2019 focalisé sur la situation contemporaine des médias en Algérie (NAQD, 1995 et 2019). La revue Tiers Monde s'est intéressée aux programmes des télévisions satellitaires arabes en Algérie, en Égypte et au Maroc (Khouri, Mignot-Lefebvre, 1996). Confluences Méditerranée a également édité un ensemble en 2007 accordant une place aux médias en Afrique du Nord: sur la Tunisie, Nozha Smati décrit un «paysage audiovisuel en mutation", et Rachid Khechana montre l'importance de l'État partisan; Kader Abderrahim analyse comment la presse algérienne est un fort enjeu de pouvoir. Un numéro d'Horizons maghrébins, coordonné par Mohamed El Oifi et Nozha Smati, propose à partir de brefs articles un état des lieux des transformations récentes des espaces médiatiques au Maghreb, en prenant également en compte les médias liés à l'immigration (El Oifi et Smati, 2010). Les soulèvements en Tunisie, en Égypte et, à un degré moindre, au Maroc ont suscité de nouvelles recherches. Par exemple, un numéro de la revue Égypte Monde arabe, proposé par Enrico de Angelis (de Angelis, 2015), s'interroge sur "l'évolution des systèmes médiatiques après les révoltes», notamment à partir de nombreux articles sur le cas égyptien et un sur une expérience d'un journal en ligne en Tunisie. L'Année du Maghreb a également pour la première fois consacré un numéro au journalisme rassemblant un ensemble 
de textes sur l'Algérie, la Tunisie et le Maroc (Chouikha et al., 2017). Les revues "d'aires culturelles» de langue anglaise publient également de plus en plus de recherches, soit sous forme de numéros thématiques (voir par exemple: Farfanfarmaian, 2014 et 2017), soit des articles au fil de l'eau.

Enfin, et plus largement, si des revues francophones très généralement liées aux seules sciences de l'information et de la communication (Communication, Les Enjeux de l'information et de la communication, Questions de communication, Réseaux et Tic \& société tout particulièrement) publient des articles sur les médias en Afrique du Nord, comme le montre notamment un numéro de Questions de communication parus récemment sur les univers de la presse écrite en Algérie (Dris, 2017), au Maroc (Benchenna et al., 2017), en Tunisie (Klaus et Koch, 2017) et en Égypte (Benaziz, 2018), les textes demeurent très souvent dispersés.

\section{Bibliographie}

ABdel RAHMAn 'Awatef, Les Problèmes de la presse et des journalistes en Égypte, Le Caire, Dar al-fikr al-araby, 1995 (en arabe).

ADIB Doss Maria, Les Talk-shows en Égypte: d'un dispositif de modernisation de l'autoritarisme à des arènes de parole dissidente: mise en perspective d'une situation révolutionnaire (25 janvier - 11 février 2011), Paris, thèse de doctorat en science politique (dir.: J.-B. Legavre et S. Ben Nefissa), Université Paris 2, 2018.

AlAOUI Mustafa, Le Journaliste et les trois rois, Rabat-Paris, Casa Express Ed. et Magellan \& Cie, 2012.

AOUCHAR Amina, La Presse marocaine dans la lutte pour l'indépendance (1933-1956), Mohammédia, Walladas, 1990.

Azızı Asmaa, «Presse des immigrés marocains: entre mobilisation politique et construction identitaire (1932-1985)", Communication \& langages, n $187,2016 a$. DOI: https://doi.org/10.4074/S033615001601108X

Azızı Asmaa, "Quand des immigrés prennent la parole: appropriation des plateformes de blogging à des fins politiques par des immigrés marocains », Tic \& société, vol. 10, n² 2-3, 2016b. DOI : https://doi. org/10.4000/ticetsociete.2168

BAIDA Jamaâ, La Presse marocaine d'expression française: des origines à 1956, Faculté des lettres et sciences humaines, Université Mohammed V de Rabat, 1996. 
BALLARINI Loïc, The Independence of the News Media: Francophone Research on Media, Economics and Politics, Basingstoke, Palgrave Macmillan, 2021.

BELKACEM Ahcène-Djaballah, «Économie de la Presse et de la Communication en Algérie: de l'aventure intellectuelle à la mésaventure économique», NAQD, n 37, 2019. DOI: https://doi.org/10.3917/naqd.037.0051

BENAZIZ Bachir, De la presse privée en Égypte: sociohistoire d'un lieu de production journalistique: les cas de l'hebdomadaire al-Dustûr et du quotidien al-Masry al-Youm (2004-2010), thèse de doctorat en sociologie, Université Paris 1.

BENAZIZ Bachir, "Fortune et infortune de la presse privée égyptienne», Questions de communication, $\mathrm{n}^{\circ} 33,2018$. DOI: https://doi.org/10.4000/ questionsdecommunication.12324

BENAZIZ Bachir, " "Journaliste-conseiller du prince" et "journaliste-justicier". Figures du journalisme et transformation de la presse écrite en Égypte», dans La Culture et ses dépendances: les productions culturelles et leurs circulations au Maghreb et au Moyen-Orient, sous la dir. d'A. Benchenna et $D$. Marchetti, Beyrouth-Rabat, Presses de l'IFPO et éditions du CJB, 2021, coll. Co-éditions.

BENCHENNA Abdelfettah, «Fragiliser la contestation: les fidèles du Makhzen à l'assaut des réseaux sociaux», Médias, n 30, 2011.

BENCHENNA Abdelfettah, "De la mobilisation identitaire à l'entreprise lucrative? Le cas du portail d'information Yabiladi.com», dans Médias et migrations dans l'espace euro-méditerranéen, sous la dir. de T. Mattelart, Paris, Mare et Martin, 2014.

BenCHENNA Abdelfettah, KsIKEs Driss, MARChETtI Dominique, «La presse au Maroc: une économie très politique: le cas des supports papier et électronique depuis le début des années 1990 », Questions de communication, n 32, 2017. DOI : https://doi.org/10.4000/questionsdecommunication.11527

BENCHENNA Abdelfettah, MARCHETTI Dominique, «Une offre sous conditions: les logiques contemporaines du champ du pouvoir marocain pour contrôler la presse nationale», dans L'Invisibilisation de la censure: les nouveaux modes de contrôle des productions culturelles (Bélarus, France, Maroc et Russie), sous la dir. de Yauheni Kryzhanouski, Dominique Marchetti et Bella Ostromooukhova, Paris, Eur'Orbem éditions, 2020, coll. Études et travaux. https://halshs.archives-ouvertes.fr/halshs-03096109

BENCHENNA Abdelfettah, MARCHETTI Dominique, "Writing between the 'red lines': Morocco's digital media landscape», Media, Culture \& Society, vol. 43, n 4, 2021. DOI: https://doi.org/10.1177\%2F0163443720972316 
BensLIMANE Mehdi, Presse «indépendante» et pouvoir: le Journal (1997-2010) promoteur du trône au Maroc: une psycho-socio-anthropologie historique du journalisme politique, thèse en science politique, Université de Grenoble-Alpes, 2015. https://tel.archives-ouvertes.fr/tel-01459214/ document

BENSMAïN Abdallah, Alors l'information ? Les journalistes parlent du journalisme... et d'eux-mêmes, Casablanca, Afrique Orient, 2015.

BERRADA Mohamed Abderrahmane, La Presse écrite au Maroc: distribution et diffusion, Éditions Stouky, 2003.

BOURDIEU Pierre, DE SAINT MARTIN Monique, "Le patronat», Actes de la recherche en sciences sociales, $\mathrm{n}^{\text {os }}$ 20-21, 1978. DOI: https://doi.org/10.3406/ arss.1978.2592

BRAHIMI Brahim, Le Pouvoir, la presse et les intellectuels en Algérie, Paris, L'Harmattan, 1989.

BRAS Jean-Philippe et CHOUIKHA Larbi (dir.), Médias et technologies de communication au Maghreb et en Méditerranée: mondialisation, redéploiements et "arts de faire», Tunis, IRMC, 2002.

CHÉRIF Dris, "La presse algérienne : une dérégulation sous contraintes: les nouvelles formes de contrôle ou la "main invisible" de l'État", Questions de communication, $\mathrm{n}^{\circ}$ 32, 2017. DOI : https://doi.org/10.4000/ questionsdecommunication.11534

CHEVALDONNÉ François, La Communication inégale: l'accès aux médias dans les campagnes algériennes, Paris, CNRS, 1981.

Chevaldonné François (dir.), «Lunes industrielles: les médias dans le monde arabe », Revue de l'Occident musulman et de la Méditerranée, n 47, 1988. https://www.persee.fr/issue/remmm_0035-1474_1988_num_47_1?secti onld=remmm_0035-1474_1988_num_47_1_2212

ChouikHa Larbi, Dris Chérif, MARChetTI Dominique, Mostefaou Belkacem, «Introduction au dossier: Profession journaliste», L'Année du Maghreb, $\mathrm{n}^{\circ} 15,2016$. DOI: http://doi.org/10.4000/anneemaghreb.2768

CHOUIKHA Larbi, «Propriétés et particularités du champ politico-journalistique en Tunisie», NAQD, n 8-9, 1995. DOI: https://doi.org/10.3917/naqd.008.0113

CHOUIKHA Larbi, «Étatisation et pratique journalistique», Revue tunisienne de communication, $\mathrm{n}^{\circ} 22,1992$.

DAOUD Zakia, Les Années Lamalif: 1958-1988: trente ans de journalisme au Maroc, Casablanca et Naples, Tarik Ed. et Senso Unico, 2007. 
DUVAL Julien, GARCIA-PARPET Marie-France, «Les enjeux symboliques des échanges économiques », Revue française de socio-économie, n 10, 2012. DOI : https://doi.org/10.3917/rfse.010.0013

De ANGELIS Enrico (dir.), "Évolution des systèmes médiatiques après les révoltes arabes: nouvelles directions de recherche», Égypte Monde arabe, $n^{\circ}$ 12, 2015. DOI: https://doi.org/10.4000/ema.3369

EL-ChAzlı Youssef, "Du "mur" à la rue: la révolte des "jeunes de Facebook" en Égypte», Médias, n 30, 2011. https://www.academia.edu/3742801/Du_ Mur_\%C3\%A0_la_rue_la_r\%C3\%A9volte_des_jeunes_de_Facebook_ en_Egypte_2008_2011_

EL ISSAWI Fatima, «Alternative Public Spaces in Hybrid Media Environments: Dissent in High Uncertainty", Journalism \& Mass Communication Quarterly, vol. 98, n 3, 2021. DOI: https://doi.org/10.1177\%2F1077699021998381

EL Kadoussi Abdelmalek, "The Perception of Self-censorship among Moroccan Journalists», The Journal of North African Studies, 2020. DOI: https://doi.org/10.1080/13629387.2020.1771310

EL KHAWAGA Dina, "La restructuration de l'audiovisuel en Égypte: I'imbrication de diverses logiques d'action", dans Mondialisation et nouveaux médias dans l'espace arabe, sous la dir. de F. Mermier, Lyon, Maisonneuve \& Larose, 2003.

EL OIFI Mohammed, Smati Nozha (dir.), «Médias au Maghreb et en milieu migratoire», Horizons Maghrébins, n 62, 2010.

Farmanfarmalan Roxane (dir.), "Special Section on Tunisia and the Media», The Journal of North African Studies, vol. 19, n 5, 2014. DOI: https://doi. org/10.1080/13629387.2014.975667

Farmanfarmaian Roxane (dir.), "Medias in Morocco», The Journal of North African Studies, vol. 22, $\mathrm{n}^{\circ} 3$, 2017. https://www.tandfonline.com/toc/ fnas20/22/3?nav=tocList

FREUND Wolfgang Slim et al., L'Information au Maghreb, Tunis, Cerès production, 1992.

GonZALEZ-QUIJANO Yves, Guaaybess Tourya (dir.), Les Arabes parlent aux Arabes. La révolution de l'information dans le monde arabe, Arles, Actes Sud, 2009.

GUAAYBESS Tourya, "Blogs, réseaux sociaux et "révolutions arabes" : du fantasme à la réalité » dans La Communication politique, sous la dir. d'A. Mercier, Paris, CNRS éditions, 2017. 
GUAAYBESS Tourya, Télévisions arabes sur orbite: un système médiatique en mutation (1960-2004), Paris, CNRS éditions, 2005. DOI: https://doi. org/10.4000/books.editionscnrs.2347

HIDASS Ahmed, L'Évolution des aspects de la circulation internationale des informations: 1920-1978, thèse de droit, Université Paris XII, 1983.

HIDASS Ahmed, "Le statut de la presse écrite au Maroc», Revue tunisienne de communication, $\mathrm{n}^{\circ} 14,1988$.

HIRSCHMAN Albert O., Exit, Voice and Loyalty: response to Decline in Firms, Organizations and States, Cambridge, Harvard University Press, 1970.

JendoubI Mehdi, "Les journalistes tunisiens: caractéristiques socioprofessionnelle», dans L'Information au Maghreb, sous la dir. de W. S. Freund, Tunis, Cerès production, 1992.

KhamIS Sahar, VAUGHN Katherin, "Online Citizen Journalism and Political Transformation in the Tunisian and Egyptian revolutions: Critical Analysis ", dans Online Journalism in Africa Trends: Practices and Emerging Cultures, sous la dir. de H.M. Mabweazara, O.F. Mudhai, J. Whittaker, Routledge, London, 2014.

KHOURI Nicole et MIGNOT-LefEBVRE Yvonne, «Les télévisions arabes à I'heure des satellites», Revue Tiers Monde, vol. 37, n 146, 1996. http://www.jstor. org/stable/23592344

KLAUS Enrique et KOCH Olivier (dir.), Médias et recompositions politiques dans Ia Tunisie post-Ben Ali, Paris, IRMC-Karthala, 2020.

KLAUS Enrique et KoCH Olivier, «Les presses privées post-2011 en Tunisie: mutations économiques et politiques», Questions de communication, $n^{\circ} 32$, 2017. DOI: https://doi.org/10.4000/questionsdecommunication.11547

Kraemer Gilles, La Presse francophone en Méditerranée, Paris, Maisonneuve \& Larose, 2001.

LECOMTE Romain, «Internet et la reconfiguration de l'espace public tunisien: le rôle de la diaspora», Tic \& société, vol. 3, nos 1-2, 2009. DOI: https:// doi.org/10.4000/ticetsociete.702

LECOMTE Romain, "Révolution tunisienne et Internet: le rôle des médias sociauX», L'Année du Maghreb, n VII, 2011. DOI: https://doi.org/10.4000/ anneemaghreb.1288

MATTELART Tristan (dir.), Médias, migrations et cultures transnationales, Louvain, De Boeck Supérieur, 2007. DOI: https://doi.org/10.3917/dbu.matte.2007.01 
MATTELART Tristan, PAPATHANASSOPOUlos Stylianos, TRAPPEL Josef, «Information and News Inequalities», dans Digital Media Inequalities: Policies against divides, distrust and discrimination, sous la dir de J. Trappel (éd.), Nordicom, 2019. https://www.diva-portal.org/smash/get/diva2:1299036/ FULLTEXT02\#page $=217$

MERMier Franck (dir.), Mondialisation et nouveaux médias dans l'espace arabe, Paris, Maisonneuve \& Larose/Maison de l'Orient et de la Méditerranée, 2003.

MIÈGE Jean-Louis, "Journaux et journalistes à Tanger », Hespéris Tamuda, $1^{\mathrm{er}} / 2^{\mathrm{e}}$ trimestre, 1954. https://www.hesperis-tamuda.com/ Downloads/1950-1959/Hesp\%C3\%A9ris\%20Tamuda\%201954.pdf

MollaRd Pierre-José, Le Régime juridique de la presse au Maroc, Rabat-Paris, Éd. La Porte et Librairie Médicis, 1963.

MostefaOU Belkacem, « Naissance du mouvement des journalistes algériens», Mediaspouvoirs, $\mathrm{n}^{\circ} 16,1989$.

Mostefaou Belkacem, «Professionnalisation et autonomie des journalistes au Maghreb. Eléments de mise en situation des actions et conflits", Réseaux, vol. 10, n 51, 1992.

Mostefaou Belkacem, La Télévision française au Maghreb: structures, stratégies, enjeux, Paris, L'Harmattan, 1995.

MudDATHIR Ahmad, Die Arabische Presse in den Maghreb-Staaten, Hambourg, Deutsches Institut für Afrika-Forschung, 1966.

NAJAR Sihem (dir.) Les Réseaux sociaux sur Internet à I'heure des transitions démocratiques, Paris, IRMC-Karthala, 2013.

NAQD, «Médias, communication et société», nos 8-9, 1995. https://www.cairn. info/revue-naqd-1995-1.htm

$N A Q D$, «Médias, communication et société II: des temps nouveaux», $\mathrm{n}^{\circ} 37$, 2019. https://www.cairn.info/revue-naqd-2019-1.htm

NAIMI Mohammed, "Liberté de presse écrite au Maroc: I'évolution au regard de l'évaluation », L'Année du Maghreb, n 15, 2016. DOI: https:// doi.org/10.4000/anneemaghreb.2786

NoËL Sophie, L'Édition indépendante critique : engagements politiques et intellectuels, Villeurbanne, Presses de l'Enssib, 2021 [2012], coll. Papiers. DOI : https://doi.org/10.4000/books.pressesenssib.1104

PIGÉ François, Radiodiffusion et télévision au Maghreb, Paris, FNSP, 1966. 
POWERS Matthew, RusseLL Adrienne, "Introduction», dans Rethinking Media Research for Changing Societies, sous la dir. de M. Powers, A. Russell, Cambridge, Cambridge University Press, 2020. DOI: https:// doi.org/10.1017/9781108886260.001

Richter Carola, Kozman Claudia (éd.), Arab Media Systems, Cambridge, UK, Open Book Publishers, 2021. DOI: https://doi.org/10.11647/OBP.0238

SALEH Souleyman, La Crise de la liberté de la presse en Égypte 1945-1985, Le Caire, La Maison d'édition des universités égyptiennes, 1995 (en arabe).

SOURIAU-HoEbreChTS Christine, La Presse maghrébine: Libye-Tunisie-MarocAlgérie, Paris, CRESM-CNRS, 1975.

TIERS Joëlle, Ruf Werner K, "La presse au Maghreb», dans Introduction à I'Afrique du Nord contemporaine, Aix-en-Provence, Institut de recherches et d'études sur les mondes arabes et musulmans, 1975. DOI: https://doi. org/10.4000/books.iremam.134.

TOUATI Zeineb, "La révolution tunisienne: interactions entre militantisme de terrain et mobilisation des réseaux sociaux», L'Année du Maghreb, 2012. DOI: https://doi.org/10.4000/anneemaghreb.1426

ZAID Bouziane, "The Authoritarian Trap in State/Media Structures in Morocco's Political Transition », The Journal of North African Studies, vol. 22, n 3, 2017. DOI: https://doi.org/10.1080/13629387.2017.1307910 


\section{Partie I}

Des dispositifs de «régulation » indépendants? 



\title{
Chapitre 1
}

\section{Le difficile déploiement des «instances indépendantes » dans la Tunisie en «transition »: le cas de la régulation de l'audiovisuel pendant les élections de 2019}

\author{
Larbi Chouikha
}

Depuis les événements du 14 janvier 2011, la "configuration étatique ${ }^{1}$ ", qui a émergé à l'indépendance du pays en mars 1956 et a perduré jusqu'à la «révolution », est en pleine mutation. L'État post-14 janvier se caractérise par la fragilité de ses institutions, l'instabilité des gouvernements, une application des lois à géométrie variable, etc. De plus, la « révolution tunisienne» "est sans leaders et sans auteurs (au sens d'acteur politique structuré placé à la tête du mouvement révolutionnaire) » (Chouikha et Gobe, 2020, p. 96), ces facteurs rendant difficile l'exercice d'une bonne "gouvernance politique» alors même que les défis socio-économiques, sécuritaires, politiques, etc. ne cessent de croître. Ils nous renseignent cependant sur l'état de la reconfiguration politique et ses prolongements dans plusieurs secteurs de la vie sociale, y compris le monde des médias.

Pour pallier ces carences, des «instances indépendantes» ont vu le jour au lendemain de la «révolution», c'est-à-dire dans une «conjoncture

\footnotetext{
${ }^{1}$ En référence aux travaux de Norbert Elias. Comme le souligne Roger Chartier dans son avantpropos à La Société des individus, le concept de configuration «permet de penser le monde social comme un tissu de relations» (Elias, 1991, p. 12). « [...] En s'appuyant sur la métaphore du jeu, Norbert Elias définit dans le terme de configuration: "la figure globale toujours changeante que forment les joueurs; elle inclut non seulement leur intellect, mais toute leur personne, les actions et les relations réciproques. [...] Cette configuration forme un ensemble de tensions..."», explique Nicolas Duvoux $(2011, \S 4)$.
} 
fluide ${ }^{2}$ », afin de conduire la "transition» dans plusieurs domaines, tout particulièrement l'organisation des élections et le fonctionnement des médias. Tout en apparaissant comme des outils indispensables de sortie de l'autoritarisme qui a régenté le pays durant plus de cinquante ans, ces instances indépendantes sont parties prenantes d'une dynamique de changements politiques qui génère des incertitudes, des calculs et des jeux d'acteurs, mettant à nu le décalage qui existe entre les textes et la pratique. Toutefois, les gouvernants tunisiens semblent désormais dans l'incapacité de se passer d'autorités indépendantes, ne serait-ce que d'un point de vue formel, car elles correspondent aux «standards internationaux» en matière de régulation démocratique (Chouikha et Gobe, 2020, p. 96).

Dans ce sillage, le processus des campagnes électorales constitue une bonne entrée pour jauger l'état des institutions, celui des lois censées les organiser et surtout le jeu des acteurs impliqués dans ces élections. C'est pourquoi l'analyse des activités de la Haute Autorité indépendante de la communication audiovisuelle (HAICA) pendant les élections présidentielle et législatives de 2019 donne la possibilité de scruter la manière par laquelle cette instance entend garantir les principes de "la neutralité des médias nationaux, la transparence de la campagne électorale quant aux sources de son financement (...) l'égalité des chances entre tous les candidats» pour reprendre les termes de l'article 52 de la loi électorale organique du 26 mai 2014 relative aux élections et aux référendums (JORT, 2014, p. 1317).

Cependant, dans sa mission régulatrice des médias audiovisuels pendant les élections, la HAICA fait face à plusieurs difficultés et écueils qui portent sur l'interprétation et la mise en application des normes adoptées: la loi sur l'audiovisuel, le Code électoral, son propre cahier des charges, les règles et procédures spécifiques pour la couverture des campagnes électorales par les médias audiovisuels et leur respect par tous les acteurs. Ces écueils se manifestent tout d'abord dans ses relations avec l'Instance supérieure indépendante pour les élections (ISIE), avec laquelle elle partage les prérogatives sur la couverture des médias pendant les élections. Ils portent ensuite sur ses rapports avec les entrepreneurs des médias privés, qui sont non seulement souvent peu scrupuleux à l'égard du respect des normes et des principes, mais également pour certains impliqués d'une manière

\footnotetext{
${ }^{2}$ Les conjonctures fluides sont définies par Michel Dobry comme des «situations incertaines où différentes ressources, relativement cloisonnées les unes aux autres dans les conjonctures routinières, viennent à être confrontées du fait des mobilisations et de la transformation corrélative des rapports entre les sites sociaux où ces ressources opèrent » (Dobry, 1986, p. 138).
} 
ou d'une autre dans les enjeux électoraux. Ces difficultés transparaissent enfin dans ses relations avec les organes gouvernementaux et étatiques (présidence du gouvernement, etc.).

Dans cette perspective, nous nous interrogerons pour savoir comment une instance publique indépendante censée réguler les médias audiovisuels durant le processus électoral où s'entremêlent plusieurs enjeux et intérêts contradictoires peut se déployer dans ces conditions. Pour la réalisation de notre travail, nous nous sommes basés à la fois sur les rapports de l'ISIE et de la HAICA, sur les déclarations publiques des responsables de ces instances, sur les entretiens que nous avons eus avec des membres de ces instances et avec des acteurs du secteur de l'audiovisuel et sur le rapport auquel nous avons contribué portant sur "la cartographie des médias en Tunisie» (ECDPM, 2020) dans le cadre d'un projet financé par l'Union européenne.

\section{Une instance indépendante pour réguler un paysage audiovisuel en pleine effervescence}

Parmi les premiers mécanismes créés après la « révolution » pour organiser le secteur de l'audiovisuel figure un dispositif légal et institutionnel (décretloi $n^{\circ} 116$ du 2 novembre 2011) qui a été adopté en novembre 2011 mais n'a été mis en application qu'une année après (cf. infra). Il stipule la création de la Haute autorité indépendante de la communication audiovisuelle, dotant pour la première fois la Tunisie d'un instrument de régulation des médias audiovisuels. Cependant, cette instance est provisoire, car elle est appelée à être remplacée par une nouvelle instance, I'Instance pour la communication audiovisuelle (ICA) qui fera partie des cinq instances constitutionnelles, conformément à la nouvelle Constitution adoptée en 2014. Inspirée entre autres des législations française en la matière, cette structure a bénéficié de l'expertise, de l'appui logistique et de financements de l'Union européenne.

Le texte du décret-loi sur l'audiovisuel attribue à la Haute Autorité la personnalité civile, l'autonomie financière et la charge « de garantir la liberté et le pluralisme de la communication audiovisuelle» (articles 3 et 6). La HAICA dispose de pouvoirs décisionnels, de contrôle et de sanction (articles 16, 17 et 18). Elle est notamment chargée, d'une part, de faire respecter les règles applicables au secteur de la communication audiovisuelle par l'ensemble des acteurs et, d'autre part, de statuer sur les demandes d'octroi des licences relatives à la création et à l'exploitation des médias audiovisuels. Elle doit également veiller à garantir la liberté d'expression et le pluralisme 
des idées et des pensées, en particulier en ce qui concerne l'information politique, tant pour le secteur audiovisuel privé que pour le secteur public. Elle dispose en outre du droit "d'avis conforme» pour les nominations à la tête des médias audiovisuels publics (article 19). Elle est composée de neuf membres, nommés pour un mandat de six ans non renouvelable (article 7). Trois membres sont désignés par le président de la République et le président de l'ANC (parlement), et les six autres membres sont élus ou désignés par le syndicat des journalistes, le syndicat général de la culture et de l'information relevant de I'UGTT (la centrale syndicale), le syndicat des directeurs des entreprises de l'audiovisuel et l'association des magistrats. La présence de magistrats a été voulue par les rédacteurs du texte dans la mesure où la HAICA constitue un premier degré de juridiction susceptible d'être amené à prendre des décisions urgentes (par exemple en période de campagne électorale).

Durant les campagnes électorales, elle doit «garantir le pluralisme et la diversité médiatiques » et "aplanir tous les obstacles juridiques et administratifs contraires au principe d'accès aux médias, sur la base de l'équité entre tous les candidats et toutes les listes électorales» (article 43). Pour ce faire, elle est chargée de veiller au respect des textes législatifs qui fixent les règles de programmation et de diffusion des séquences relatives aux campagnes électorales et, dans tous les cas, elle dispose du pouvoir de sanctionner les infractions commises par les entreprises audiovisuelles.

Les gouvernements qui se sont succédé depuis les élections d'octobre 2011 étaient frileux à l'idée de se désengager complètement d'un secteur qui fut, jadis, instrumentalisé, tant sous Bourguiba que sous Ben Ali (Chouikha, 2015, p. 15-44). C'est la raison pour laquelle ce décretloi ( $\left.n^{\circ} 116\right)$ n'a été exécuté qu'une année après sa publication au Journal officiel (novembre 2011). C'est en effet sous la pression des journalistes et à la suite d'une grève générale qu'ils venaient d'observer le 17 octobre $2012^{3}$ sur tout le territoire et dans tous les médias - une première en Tunisie que le gouvernement de la troïka ${ }^{4}$ annonça la mise en œuvre du décret-loi le jour même. Quant à la composition du conseil de la HAICA, elle n'a été

\footnotetext{
${ }^{3} \mathrm{Cf}$. le communiqué du Syndicat national des journalistes tunisiens (SNJT) appelant à la grève générale le 14 octobre 2012 pour dénoncer les atteintes à la profession et pour revendiquer une protection juridique et une amélioration des conditions morales et matérielles.

${ }^{4}$ Une coalition au pouvoir constituée au lendemain des élections à la Constituante le 23 octobre 2011 formée essentiellement du mouvement islamiste Ennahdha et de ses deux alliés «laïcs», de moindre importance numérique, le Congrès pour la République (CPR) et le parti Ettakatol.
} 
rendue publique que le 3 mai 2013, c'est-à-dire une année et demie après la publication du décret, après moult tractations portant sur le choix des membres entre les composantes formant la troïka.

Dix mois après son démarrage et à quelques semaines des élections législatives et présidentielles d'octobre 2014, la HAICA rend publique la première liste des stations de radio et des chaînes de télévision - privées et associatives - autorisées à émettre ou à diffuser. Conformément à son cahier des charges (HAICA, 2014), la HAICA a refusé d'emblée des autorisations aux demandes émanant de promoteurs travaillant dans la publicité et la communication, de membres des instances dirigeantes de partis politiques (Z.A., 2014).

\section{| Un paysage audiovisuel tiraillé}

Fin 2020, le paysage audiovisuel comprend 12 chaînes de télévision (2 publiques et 10 privées, y compris les deux chaînes non autorisées par la HAICA (cf. infra), et plus d'une quarantaine de stations de radio réparties en stations publiques (10), stations commerciales privées (18) et stations de radio associatives $(22)^{5}$. Par rapport à la situation antérieure au 14 janvier 2011, ce paysage audiovisuel se singularise par trois caractéristiques: une forte intrusion des hommes d'affaires et des acteurs politiques dans le monde des médias, des médias diffusant en toute impunité sans licence et un État qui demeure le principal propriétaire dans ce secteur tout en ayant des ressources très limitées.

\section{Intrusion des hommes d'affaires et opacité financière}

Sous l'ex-président Ben Ali, des hommes d'affaires influents ont reçu I'autorisation de lancer des médias privés, notamment audiovisuels, mais les conditions posées par le pouvoir politique à l'époque limitaient considérablement leurs ambitions personnelles (Chouikha, 2010). Avec l'avènement de la «révolution», les accointances du monde des affaires et de la politique avec celui des médias se sont renforcées et généralisées

\footnotetext{
${ }^{5}$ Voir le site de la HAICA : http://haica.tn/\%D9\%81\%D8\%B6\%D8\%A7\%D8\%A1-\%D8 \%A7\%D9\%84\%D9\%85\%D9\%87\%D9\%86\%D9\%8A\%D9\%8A\%D9\%86/\%D8\%A7\%D9\%8 4\%D9\%82\%D9\%86\%D9\%88\%D8\%A7\%D8\%AA-\%D8\%A7\%D9\%84\%D8\%A5\%D8\%B0\%D8\%A7\%D8\%B9\%D9\%8A\%D8\%A9-\%D9\%88\%D8\%A7\%D9\%84\%D8\%AA\%D9\%84\%D9\%81\% D8\%B2\%D9\%8A\%D8\%A9/\#firstPage/first Page
} 
- souvent dans la confusion - sous l'effet de deux facteurs très imbriqués: d'une part, l'émergence d'un climat de liberté qui s'accompagne du recul de l'«État autoritaire» qui a régenté le pays de l'Indépendance à janvier 2011; d'autre part, l'ambition exprimée par plusieurs entrepreneurs de se lancer dans la vie politique en investissant dans les médias audiovisuels. Pourtant, comme nous l'avons mentionné précédemment, le cahier des charges de la HAICA érige des balises pour protéger les médias audiovisuels de leur intrusion en interdisant notamment le titulaire d'une licence d'assumer des responsabilités partisanes, d'être à la tête d'un institut de sondage ou d'une agence de publicité. II ne peut par ailleurs cumuler plus d'une station de radio et une chaîne de télévision à la fois.

Pour autant, la question de la transparence des sources de financement des grands médias audiovisuels se pose avec d'autant plus d'acuité que les recettes publicitaires qui alimentent ces médias (mais dont personne ne connaît le montant exact) ne suffisent pas à couvrir leurs besoins. Par conséquent, comme le montre l'étude réalisée sur ce sujet par Reporters sans frontières (RSF) et l'association Al Khatt, qui édite le journal électronique d'investigation Inkyfada (RSF-Al Khatt, 2016), I'opacité qui entoure les sources de financement dans la plupart des médias audiovisuels privés constitue une réelle menace pour leur indépendance.

\section{Des médias qui diffusent sans licence}

La deuxième particularité du paysage audiovisuel tunisien contemporain, c'est l'existence de chaînes de télévision et de stations de radio qui émettent ou diffusent sans l'autorisation de la HAICA. La plus emblématique est Ezzitouna TV, créée en 2012 par le fils d'un ancien ministre d'Ennahdha (mouvement islamiste) de la troïka. Celui-ci ne peut légalement prétendre à une autorisation, du fait de son appartenance à une instance dirigeante d'un parti politique, Ennahdha. La menace de la HAICA de confisquer le matériel des chaînes et des stations qui ne disposent pas de licences, parmi lesquelles Ezzitouna TV (African manager, 2015), a suscité une vive réaction de la part du leader du mouvement, Rached Ghannouchi, dans un communiqué du 2 février 2015, et la chaîne continue de diffuser en toute quiétude.

Le cas de Nessma TV, dont le propriétaire, Nabil Karoui, fut candidat aux dernières élections présidentielles (2019), est encore plus flagrant. Cette chaîne de télévision cumule une forte audience, du fait surtout de la diffusion de feuilletons turcs et arabes à l'instar de "Harim Al Soltan» portant sur la vie amoureuse du sultan Soliman qui, en rupture avec les 
traditions, épousa l'une des filles de son harem. Cette chaîne a été créée sous l'ex-président Ben Ali en mars 2007, et, en mai 2008, le groupe publicitaire tunisien Karoui \& Karoui World propriétaire de la chaîne a annoncé l'entrée dans le capital du groupe italien Mediaset, dont le principal actionnaire est l'ex-Premier ministre italien Silvio Berlusconi, et du groupe tunisien Quinta Communications de Tarak Ben Ammar (gendre de l'ex-épouse de Bourguiba) et ce, à hauteur de $25 \%$ chacun. Nessma TV diffuse aujourd'hui en toute illégalité en raison de son refus de se conformer aux dispositions fixées par la HAICA (Leaders, 2019).

Nabil Karoui est devenu ensuite président d'un parti initialement fondé le 20 juin 2019 et qui porte désormais le nom de Qalb Tounés (cœur de Tunisie). À l'issue des élections législatives de 2019, son parti est devenu la seconde formation au parlement (38 députés sur 217). Alors que la loi le lui interdit, il a mis à son profit sa chaîne de télévision et son association, qui porte le nom de son fils décédé dans un accident, qui distribue des repas et des aides diverses aux villages pauvres de la Tunisie intérieure, en le faisant savoir via des reportages télévisés. Quant aux stations de radio, Al Qûran Al Karim, dont le propriétaire, Saïd Jaziri, est un prosélyte religieux et chef du parti Errahma, groupuscule religieux à vocation piétiste, est considérée par la HAICA comme une "radio-pirate » puisqu'elle opère sans licence et recourt à des émetteurs acquis en dehors des circuits officiels.

Face aux agissements des dirigeants de médias qui diffusent sans licence, la HAICA ne dispose que d'un pouvoir de sanction qui se limite à suspendre des émissions et à prononcer des amendes contre les propriétaires réfractaires. Elle n'a pas de moyens coercitifs pour saisir le matériel et fermer les entreprises médiatiques en situation illégale. Son seul et unique recours repose sur la volonté des gouvernants de mettre à exécution ses décisions de fermeture. Or, tous les gouvernements qui se sont succédé depuis la «révolution » refusent ou hésitent à mettre en œuvre ces sanctions, de peur d'être accusés de vouloir attenter à la liberté d'expression ${ }^{6}$. De plus, toutes les chaînes de télévision, y compris celles qui diffusent sans autorisation légale, le font via les satellites de télédiffusion directe et, pour la radio-pirate,

\footnotetext{
${ }^{6}$ Le gouvernement de Youssef Chahed avait tenté de mettre à exécution la décision de la HAICA en voulant fermer la chaîne Nessma TV en avril 2019, c'est-à-dire à la veille des élections. Mais la décision du chef du gouvernement était surtout mue par sa rivalité avec le propriétaire de la chaîne, Nabil Karoui, candidat comme lui à l'élection présidentielle. Chahed s'est aussitôt rétracté sous la pression des syndicats et des leaders politiques. Sur ce sujet, voir Dahmani, 2019b.
} 
via des émetteurs non accordés par l'organisme officiel, I'Office national de la télédiffusion (ONT).

\section{L’État, principal propriétaire}

La troisième caractéristique du paysage audiovisuel est que l'État tunisien reste le principal propriétaire. Outre le fait qu'il détient déjà deux chaînes de télévision, Wataniya 1 et Wataniya 2, et l'Établissement de la radio tunisienne qui gère une dizaine de stations de radio réparties sur l'ensemble du territoire, il est actionnaire dans plusieurs organes médiatiques privés, dont les parts revenant à la famille de Ben Ali ont été confisquées, comme les stations de radio généralistes Shems FM et religieuse Zitouna FM.

Mais la situation économique et sociale de la plupart de ces entreprises, aussi bien publiques que celles qui ont été confisquées, est devenue précaire. En effet, plusieurs d'entre elles connaissent des difficultés inhérentes aux multiples problèmes hérités de la période Ben Ali (sureffectif, mauvaise gestion, etc.), et tous les gouvernements qui se sont succédé depuis le 14 janvier 2011 hésitent à engager des réformes drastiques, de peur de provoquer des mouvements sociaux au sein de ces médias.

\section{| La HAICA à l'épreuve des élections de 2019}

La campagne pour les élections présidentielle et législatives en 2019 s'est déroulée dans un contexte politique agité, du fait d'événements inattendus qui ont pesé sur le processus électoral. En effet, à quelques semaines du démarrage de la campagne, deux faits marquants sont survenus brutalement: le décès du chef de l'État, Béji Caid Essebsi (25 juillet), et l'incarcération dans l'attente de son procès du propriétaire de Nessma TV, candidat à la présidentielle, Nabil Karoui (23 août).

Le décès du président de la République créa une nouvelle donne, conformément à l'article 84 de la Constitution. Il signifiait l'inversion du calendrier des élections (pour la première fois, l'élection présidentielle précédait celle des législatives) et modifiait les stratégies électorales des principales formations politiques en compétition. Les instances qui supervisent les élections, I'ISIE et la HAICA, adaptèrent sans difficulté leur programme en fonction du nouveau calendrier électoral. 
Cependant, l'arrestation et l'incarcération de Nabil Karoui le 23 août 2019 en vertu d'un mandat de dépôt émis dans la matinée par la chambre d'accusation de la cour d'appel de Tunis (Bobin, 2019) ont mis dans l'embarras ces deux instances. Nabil Karoui et son frère Ghazi étaient déjà interdits en juillet 2019 de quitter le territoire national. Ils avaient également vu leurs avoirs gelés sur décision du juge d'instruction du pôle judiciaire, économique et financier à la suite d'une plainte déposée en 2016 par I'ONG anti-corruption «I Watch» (Dahmani, 2019).

Toutefois, Nabil Karoui, élu, a été mis en liberté provisoire entre les deux tours de la présidentielle et a pu se présenter pour le second tour, sa candidature ayant été validée par l'instance des élections, I'ISIE, dont le président a expliqué que «tant qu'il n'y a pas un verdict pour priver un individu de se présenter à la présidentielle, l'instance ne peut refuser sa candidature» (E.B.A., 2019). De même, la HAICA, conformément au principe "de l'égalité de traitement entre les candidats» (art. 52 du Code électoral), a permis à Nabil Karaoui de participer aux débats télévisés du second tour (webmanagercenter, 2019).

Toutefois, la gestion de cette affaire a révélé des tensions latentes entre les deux instances, qui ont rejailli pendant la campagne et à l'annonce des résultats officiels.

\section{Des relations difficiles avec I'ISIE}

Dans les conditions politiques et institutionnelles difficiles où se sont déroulées les campagnes électorales de 2019, des problèmes ont surgi entre la HAICA et I'ISIE, se cristallisant à la fois autour de la délimitation du champ de compétence de chaque instance en période électorale et sur l'appréciation que chacune d'elle porte aux lois et aux principes qui régissent les élections. Ces questions ont des prolongements dans les structures de l'État et chez les acteurs politiques. Dans ce contexte tendu, le défi qui se pose à la HAICA est le suivant: comment réguler un processus électoral marqué par l'absence de leadership politique dans le pays après le décès de l'ex-président (25 juillet 2019) et surtout par l'absence d'une Cour constitutionnelle, pourtant pièce maîtresse du dispositif électoral, du fait de la mésentente des grands partis?

Ces facteurs pèsent dans ses rapports avec I'ISIE, et un différend a surgi aussitôt à propos de la prise en compte ou non des médias qui ne disposent pas d'une licence de la HAICA. Ce différend porte sur les dispositions de l'article 5 de l'accord sur «les règles et procédures spécifiques pour 
la couverture des campagnes électorales par les médias audiovisuels», signé par les deux instances le 21 août 2019 (ISIE, 2019, p. 294). Cet article controversé stipule qu'«il n'est pas permis de faire campagne à travers les médias audiovisuels qui exercent leur activité de diffusion en dehors du cadre du décret-loi $n^{\circ}$ 2011-116 et sans avoir obtenu de licence». Pour la HAICA, cette formulation est délibérément ambiguë, voire accommodante, pour les médias illégaux; il fallait tout bonnement leur interdire de couvrir les campagnes électorales à l'instar de Nessma TV, Zitouna TV et la radio Al Qûran Al Karim. Alors que, pour I'ISIE, l'interdiction revenait à les exclure de leur champ de surveillance, et, de plus, leur interdiction d'émettre n'a jamais été exécutée par les gouvernements successifs ${ }^{7}$. Ce différend a pesé lourd sur le processus électoral et a encore altéré les relations entre les deux instances.

La HAICA a publié trois rapports de monitoring sur le contrôle de la couverture médiatique de la campagne électorale et a infligé au total 45 amendes à plusieurs médias pour "violation des règles concernant les médias» (Mission d'observation électorale de l'Union européenne, 2019, p. 38). Les motifs portent sur les activités interdites telles que la propagande politique, la violation du silence électoral et l'évocation des sondages (HAICA, 2019a). Toutefois, le principal grief formulé par la HAICA pendant la campagne se focalise sur la participation des propriétaires des médias aux compétitions électorales et l'implication des médias en faveur des candidats (HAICA, 2019b). Dans cette optique, elle insiste pour que I'ISIE sanctionne ces médias comme la loi électorale l'y autorise expressément (JORT, 2014, p. 1328).

\section{Des candidats propriétaires ou actionnaires dans les médias}

En effet, comme on l'a mentionné précédemment, plusieurs propriétaires de médias audiovisuels sont des hommes d'affaires, responsables ou membres des instances dirigeantes de partis politiques, ce qui constitue une infraction aux dispositions légales. Or, aux dernières élections en 2019, des candidats aux élections présidentielle et législatives «avaient des liens directs (propriété, copropriété, actionnariat, etc.) avec des médias audiovisuels privés, ayant donc la possibilité de les influencer à des fins de propagande et de soutien ", selon la HAICA (Mission d'observation électorale de l'Union européenne, 2019, p. 10 et note 24, p. 8).

\footnotetext{
${ }^{7}$ Selon les propos du président de l'ISIE tenus à l'auteur (avril 2020).
} 
C'est particulièrement le cas de la chaîne Nessma TV, qui est devenue un organe de propagande politique au service de son propriétaire, Nabil Karoui, candidat à l'élection présidentielle et leader d'un parti politique (Qalb Tounés) au nom duquel il présenta des listes électorales aux législatives. La chaîne a consacré 24 heures et demi du temps de diffusion de la campagne électorale à la propagande pour le parti Qalb Tounés sur un total d'environ 27 heures, et plusieurs sanctions lui ont été infligées par la HAICA (HAICA, 2019b).

La radio-pirate $A$ l Qûran Al Karim, qui opère sans licence, constitue un cas atypique. Cette radio a largement fait de la propagande pour le parti Errahma, que dirige son propriétaire Saïd Jaziri, lors des dernières élections législatives. D'ailleurs, il a été élu député dans la circonscription de Ben Arous (banlieue-sud de Tunis), qui est entièrement couverte par cette radio locale. Pour sa part, la chaîne Zitouna TV, qui opère également sans licence de la HAICA, a consacré de larges séquences aux activités du candidat d'Ennahdha aux élections présidentielles, Abdelfattah Mourou.

D'autres propriétaires de chaînes ont mis leurs médias au service de personnalités à des fins de propagande politique. C'est principalement le cas de la chaîne El Hiwar Ettounsi, que dirige un personnage controversé, Sami El Fehri. En novembre 2019, il a été arrêté pour «soupçon de blanchiment d'argent». Sous l'ex-président Ben Ali, il avait fondé en 2002 avec le beaufrère de ce dernier, Belhassen Trabelsi, la société de production télévisuelle "Cactus Prod» (Jeune Afrique avec AFP, 2019). Au cœur de la campagne présidentielle, sa chaîne El Hiwar Ettounsi a réalisé une interview de deux heures et demie avec le candidat à la présidentielle, l'homme d'affaires exilé en France Slim Riahi ${ }^{8}$, toujours en infraction avec les règles de la HAICA (Gnetnews, 2019).

Conformément à la loi électorale qui donne les pleins pouvoirs à I'ISIE pour décider l'annulation totale ou partielle des résultats (JORT, 2014, p. 1328), la HAICA lui a communiqué la liste des infractions commises par ces chaînes et lui a demandé de sanctionner les propriétaires suivants : Nabil Karoui (Nessma TV), Oussama Ben Salem (Zitouna TV) et Saïd Jaziri (Al Qûran Al Karim). Aussi, seule la liste du parti Errahma - de la circonscription de Ben Arous conduite par Saïd Jaziri - a été invalidée dans un premier temps par I'ISIE pour «infractions aux règles électorales», avant qu'elle

\footnotetext{
${ }^{8}$ La cour d'appel de Tunis avait émis, le 17 avril 2019, un mandat de dépôt à son encontre, et son dossier a été transféré à la chambre criminelle spécialisée dans les affaires de corruption financière pour des soupçons de blanchiment d'argent (Nemlaghi, 2019).
} 
ne soit réhabilitée par les autorités judiciaires (M.K.J., 2019). La décision d'invalidation de la part de I'ISIE se basait sur le fait que les candidats de cette liste partisane appartiennent tous à la même circonscription couverte par la radio Al Qûran Al Karim? .

Cependant, la justice a débouté I'ISIE, jugeant qu'il n'y avait pas de preuve matérielle qui atteste que l'élection des candidats du parti Errahma résulterait de «l'influence directe» de cette radio sur les électeurs de cette circonscription ${ }^{10}$. Et depuis sa réhabilitation, le propriétaire de la station mène une campagne contre la HAICA (Jaoudi, 2020). Pour les deux autres propriétaires des médias incriminés, qui n'ont pas fait l'objet de sanction de la part de I'ISIE, Nessma TV et Zitouna TV, la HAICA n'a pu que manifester publiquement son mécontentement à travers un de ses membres qui «s'étonne de la passivité et du manque d'impartialité de I'ISIE» (Ben Younes, 2019).

En fait, les dissensions qui ont émaillé les relations entre les deux instances sont essentiellement liées au contexte général qui a prévalu tout au long des campagnes pour les élections de 2019. Ce contexte est marqué par une lutte âpre entre les chefs des partis politiques et les hommes d'affaires, et, dans ces conditions, les grands médias - essentiellement télévisuels ne sont pas en reste: ils sont partie prenante en s'alignant sur les intérêts et les accointances politico-affairistes de leurs propriétaires; ils font la part belle aux discours de leur candidat favori et dénigrent ouvertement les concurrents sans que l'instance de régulation ne puisse réagir avec fermeté.

Rappelons que la HAICA ne dispose pas de moyens directs pour faire pression sur les propriétaires récalcitrants en cas d'infraction. D'une part, la loi régissant le secteur de l'audiovisuel lui octroie un pouvoir de sanction financière qui est loin d'être dissuasif pour les propriétaires de ces médias; peu d'entre eux daignent s'en acquitter ${ }^{11}$. D'autre part, le peu d'empressement des structures de l'État à collaborer efficacement avec la HAICA lui ôte tout pouvoir de contrainte et amenuise son autorité aux yeux des propriétaires des médias audiovisuels. Depuis le 14 janvier 2011, les gouvernements successifs ne sont pas très enclins à vouloir appliquer les décisions de la HAICA portant sur la fermeture des médias illégaux.

\footnotetext{
${ }^{9}$ Selon les propos du président de I'ISIE tenus à l'auteur (avril 2020).

10 Décision du tribunal administratif, «Affaire 20194053 », 31 octobre 2019 (en arabe).

${ }^{11}$ Selon des membres de la HAICA, pour l'exercice des années 2018 et 2019, moins de $30 \%$ des amendes infligées aux médias en infraction ont été versées au Trésor public (propos recueillis par l'auteur en mai 2020).
} 
Il convient de souligner en 2021, c'est-à-dire dix ans après les événements de janvier 2011, que l'État tutélaire issu des années de l'Indépendance est en pleine mutation, et il serait hasardeux de conjecturer sur l'issue des évolutions en cours. "La sociologie historique de Norbert Elias» est utile parce que, comme l'écrit Florence Delmotte, "une sociologie «des configurations» est forcément une sociologie "des processus», et ces deux ambitions ne sont d'ailleurs jamais distinguées» (Delmotte, 2010, p. 33). Cependant, du fait de la fragilité des institutions publiques et de la vulnérabilité des lois censées superviser et baliser le processus politique en Tunisie, des lobbies et hommes d'affaires peu scrupuleux cherchent à se nicher dans des "entités invisibles » (Brésillon, 2018) pour noyauter des structures étatiques. Dans leur sillage, non seulement les enjeux politico-personnels et les logiques particularistes (affairistes, religieux, politiques, régionalistes, etc.) peuvent trouver un terrain propice pour agir sur les politiques publiques, mais les calculs des acteurs politiques à la veille des élections peuvent également s'entremêler dans le cours du processus électoral (Chouikha, 2019). Dans cette logique, les médias audiovisuels nationaux ne sont pas en reste. Théoriquement, ils sont censés être «neutres» pendant la campagne électorale, mais plusieurs d'entre eux n'échappent pas à la pression des puissances de l'argent et servent les logiques de ces acteurs.

Une autre raison susceptible d'expliquer l'éclosion et le développement des logiques affairistes et personnelles sur les institutions formelles est que le monde des médias n'est pas isolé du reste de la société, et la polarisation qui s'empare de la société tunisienne depuis les élections à l'Assemblée nationale constituante en octobre 2011 a engendré des tensions (Gobe, Chouikha, 2014). Les discours et les pratiques des fractions dirigeantes à propos de l'instauration d'un régime démocratique ne font qu'entretenir cette ambiguïté : si bon nombre d'anciens militants et opposants sous Ben Ali y aspirent, certains des anciens bénéficiaires du régime de Ben Ali revenus au pouvoir à la faveur des élections législatives de 2014, porteurs de schémas et de pratiques autoritaires, ne sont pas résolus à tourner définitivement la page ${ }^{12}$. Les leçons portant sur les implications des personnes dans le système qui a régenté les médias et l'information sous Ben Ali n'ont jamais été tirées au grand jour ${ }^{13}$. Leur ambivalence vis-à-vis

\footnotetext{
${ }^{12}$ Le processus de justice transitionnelle enclenché en mai 2014 divise les Tunisiens sur les modalités de son fonctionnement et sur son issue possible.

${ }^{13} \mathrm{Cf}$. "Pour la vérité sur le système Abdelwahab Abdallah ", Déclaration à l'occasion du $59^{\mathrm{e}}$ anniversaire de la République tunisienne en juillet 2016. A. Abdallah, ex-enseignant à
} 
du processus politique actuel joue de la bipolarisation de la scène publique entre islamistes et sécularistes. Elle s'en est même alimentée et a parfois contribué à l'amplifier. Qui plus est, dans leurs tentatives de dérégulation et de fragilisation des structures de l'État, ces élites politiques liées à l'ancien régime sont «fortement présentes au sein de l'exécutif depuis les élections de 2014; elles souhaitent transformer les autorités indépendantes en institutions de décor, ornements nécessaires de régimes se prévalant dans leurs discours de la démocratie et de l'État de droit » (Chouikha, Gobe, 2020, p. 114).

Cela étant, les conditions "d'indépendance» et de "compétence» reconnues aux membres des instances constitutionnelles, à l'instar de la future institution amenée à remplacer la HAICA, risquent d'être mises à mal par le nouveau mode de désignation des membres énoncé dans la Constitution promulguée le 27 janvier 2014. La HAICA est en effet appelée à se muer en une Instance de la communication audiovisuelle constitutionnelle (art. 125). À l'instar des collèges des instances constitutionnelles (art. 127), ses membres seront élus à une majorité renforcée par le Parlement, et, par conséquent, elle reflétera, pour partie, les rapports de force politique au sein de l'assemblée législative.

En dépit des menaces et défis, l'existence d'une instance de régulation de l'audiovisuel est un acquis qu'il convient de conserver et de renforcer, mais le bon fonctionnement d'une telle instance dépend d'abord de "la logique de la gouvernance [qui] affecterait en tout premier lieu l'État » (Chevalier, 2003, p. 203-217). Celle-ci implique un minimum de confiance réciproque entre les différents acteurs du paysage de l'audiovisuel et entre les gouvernants et les gouvernés, ainsi qu'une bonne compréhension de la régulation. Elle exige aussi une transparence dans la gestion et le fonctionnement interne des instances publiques, d'autant plus que les sommes qui leur sont allouées chaque année par l'État sont jugées exorbitantes par certains (B.O., 2017).

\section{Bibliographie}

CHEVALIER Jacques, "La gouvernance, un nouveau paradigme étatique?», Revue française d'administration publique, $\mathrm{n}^{\circ}$ 105-106, 2003. DOI: https:// doi.org/10.3917/rfap.105.0203

I'Institut de presse de Tunis (IPSI), fut l'architecte du système médiatique sous Ben Ali. Voir: https://www.petitions.fr/pour_la_verite_sur_le_systeme_abdelwahab_abdallah 
CHOUIKHA Larbi, «L'audiovisuel en Tunisie: une libéralisation fondue dans le moule étatique», L'Année du Maghreb, n² 2, 2005-2006, mis en ligne le 8 juillet 2010. DOI: https://doi.org/10.4000/anneemaghreb.165

ChoulKHA Larbi, La Difficile transformation des médias : des années de l'indépendance à la veille des élections de 2014, Tunis, Éditions Finzi, 2015.

CHOUIKHA Larbi, «Le processus électoral tunisien en 2019: instabilité institutionnelle et jeu des acteurs», Revista de Estudios Internacionales Mediterráneos, n 27, 2019. DOI: https://doi.org/10.15366/reim2019.27.011

CHOUIKHA Larbi, Gobe Éric, "Politique de l'indépendance en conjoncture politique fluide: le cas de la Tunisie post-Ben Ali», dans Politique de l'indépendance: formes et usages contemporains d'une technologie de gouvernement, sous la dir. de Bastien François et Antoine Vauchez, Paris, Éditions du Septentrion, 2020. DOI: https://doi.org/10.4000/books. septentrion.77851

DOBRY Michel, Sociologie des crises politiques: la dynamique des mobilisations

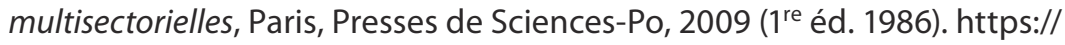
www.cairn.info/sociologie-des-crises-politiques--9782724611250.htm

DeLMOTTE Florence, «Termes clés de la sociologie de Norbert Elias», Vingtième Siècle, Revue d'histoire, vol. 2, n 106, 2010. DOI: https://doi.org/10.3917/ vin.106.0029

Duvoux Nicolas, "Configuration», Sociologie, Les 100 mots de la sociologie, mis en ligne le $1^{\mathrm{er}}$ juin 2011. http://journals.openedition.org/sociologie/923

ElIAS Norbert, La Société des individus, Paris, Fayard, 1991.

GoBE Éric, Chouikha Larbi, «La Tunisie politique en 2013 : de la bipolarisation idéologique au " consensus constitutionnel »?», L'Année du Maghreb, $\mathrm{n}^{\circ}$ 11, 2014. DOI: https://doi.org/10.4000/anneemaghreb.2340

\section{Sources}

AFRICAN MANAGER, "Tunis: la HAICA menace de confisquer le matériel de trois radios et trois télévisions », 31 janvier 2015. https://africanmanager. com/tunis-la-haica-menace-de-confisquer-le-materiel-de-trois-radioset-trois-televisions/

BEN YOUNĖS Cherif, «Élections: Hichem Snoussi s'étonne de la passivité et du manque d'impartialité de I'ISIE », Kapitalis.com, 17 octobre 2019. http://kapitalis.com/tunisie/2019/10/17/elections-hichem-snoussi-haicasetonne-de-la-passivite-et-du-manque-dimpartialite-de-lisie/ 
BoBIN Frédéric, «En Tunisie, l'arrestation du candidat Nabil Karoui bouleverse la campagne présidentielle», Le Monde, 23 août 2019. https://www.lemonde. fr/afrique/article/2019/08/23/en-tunisie-le-candidat-a-la-presidentiellenabil-karoui-arrete_5502253_3212.html

BRÉSILLON Thierry, «Tunisie: faux coup d'État, vraie crise politique», Orient XXI, 3 juillet 2018. https://orientxxi.info/magazine/tunisie-faux-coup-d-etatvraie-crise-politique, 2533

B.O., "Ces instances qui coûtent cher aux contribuables», Espace Manager, 16 mai 2017, https://www.espacemanager.com/ces-instances-quicoutent-cher-aux-contribuables.html

DAHMANI Frida, «Tunisie: Nabil Karoui, interdit de voyager et ses avoirs gelés, dénonce une "honte pour l'État de droit" ", Jeune Afrique, 9 juillet 2019. https://www.jeuneafrique.com/801093/societe/tunisie-nabil-karouiinterdit-de-voyager-et-ses-avoirs-geles-denonce-une-une-honte-pourletat-de-droit/

DAHMANI Frida, "Tunisie : fermeture de Nessma TV pour diffusion illégale», Jeune Afrique, 25 avril 2019. https://www.jeuneafrique.com/767323/ politique/tunisie-les-autorites-ordonnent-larret-de-nessma-tv-pourdiffusion-illegale/

E.B.A., «Baffoun: rien n'empêche le maintien des candidatures de Nabil Karoui et Slim Riahi », Business News, 31 août 2019. https://www.businessnews. com.tn/baffoun--rien-nempee-le-maintien-des-candidatures-de-nabilkaroui-et-slim-riahi,520,90596,3

GNETNEWS, «Interview de Slim Riahi: El-Hiwar Ettounsi écope d'une amende de 50 mille dinars», 7 septembre 2019. https://news.gnet.tn/interviewde-slim-riahi-el-hiwar-ettounsi-ecope-dune-amende-de-50-mille-dinars/

HAICA, Décisions de la Haute Autorité indépendante de la communication audiovisuelle, $\mathrm{n}^{\circ}$ 4, 5 mars 2014. http://haica.tn/espace-professionnels/ cahiers-de-charges/

HAICA, Rapport de monitoring des médias de la campagne électorale pour les législatives (du 14 septembre au 4 octobre 2019), Tunis, 2019a. http://haica. tn/media/Document000.pdf

HAICA, Rapport sur les graves infractions enregistrées pendant la campagne électorale des législatives de la part des médias non légaux suivants: les chaînes de télévision Nessma TV et Ezzitouna TV et la station de radio Al Qûran Al Karim, Tunis, 2019b (en arabe), http://haica.tn/media 
INSTANCE SUPÉRIEURE INDÉPENDANTE POUR LES ÉLECTIONS (ISIE), Recueil des textes juridiques relatifs aux élections et référendum, Tunis, septembre 2019. http://www.isie.tn/wp-content/uploads/2019/10/Recueil-des-textesrelatifs-aux-\%C3\%A9lections_Fr_2019.pdf

JAOUADI Ridha, "La HAICA mène des actions contre tout ce qui touche à I'islam et au Coran », kapitalis.com, 25 janvier 2020. http://kapitalis.com/ tunisie/2020/01/25/ridha-jaouadi-la-haica-mene-des-actions-contre-toutce-qui-touche-a-lislam-et-au-coran/?fbclid=IwAR1cGhdMLgazWa7VYGW fN1RPcHNeMK0hNp3EbiigvFmEh19zLr5nHVVw8JM

JEUNE AFRIQUE avec AFP, «Tunisie : arrestation de Sami Fehri, patron de la chaîne de télévision Elhiwar Ettounsi », 6 novembre 2019. https://www. jeuneafrique.com/852517/politique/tunisie-arrestation-de-sami-fehripatron-de-la-chaine-de-television-elhiwar-ettounsi/

JOURNAL OFFICIEL DE LA RÉPUBLIQUE TUNISIENNE (JORT), Loi électorale organique $\mathrm{n}^{\circ} 16$ en date du 26 mai 2014, relative aux élections et aux référendums, n 42, 27 mai 2014. http://www.isie.tn/wp-content/uploads/2018/01/loiorganique-n\%C2\%B02014-16.pdf

LEADERS, "La Haica met en garde Karoui contre les conséquences de I'instrumentalisation de sa chaîne à des fins politiques», 29 mai 2019. https://www.leaders.com.tn/article/27205-la-haica-met-en-garde-karouicontre-les-consequences-de-l-instrumentalisation-de-sa-chaine-a-desfins-politiques

MISSION D'OBSERVATION ÉLECTORALE DE L'UNION EUROPÉENNE, Tunisie 2019, Rapport final, Élection présidentielle, 15 septembre et 13 octobre 2019: élections législatives, 6 octobre 2019, Bruxelles, Union européenne. https://eeas. europa.eu/sites/default/files/rapport_final_moe_ue_tunisie_dec_2019_ fr.pdf

M.K.J., "Saïd Jaziri "redevient député" : I'ISIE fera appel de la décision du TA», Webdo, 22 octobre 2019. https://www.webdo.tn/2019/10/22/said-jaziriredevient-depute-lisie-fera-appel-de-la-decision-du-ta/\#.YEEF4NzfuUk

NEMLAGHI Yüsra, "Législatives 2019: I'ISIE valide la liste électorale présidée par Slim Riahi, suspecté de corruption et en fuite en France», Kapitalis. com, 7 août 2019. http://kapitalis.com/tunisie/2019/08/07/legislatives2019-lisie-valide-la-liste-electorale-presidee-par-slim-riahi-suspecte-decorruption-et-en-fuite-en-france/

WEBMANAGERCENTER (Rédaction), «Présidentielle 2019 [2 tour] : la HAICA pour la participation de Nabil Karoui à des débats télévisés», 21 septembre 2019. https://www.webmanagercenter.com/2019/09/21/439171/presidentielle- 
2019-2eme-tour-la-haica-pour-la-participation-de-nabil-karoui-a-desdebats-televises/

Reporters SANS FRONtIÈres, Al KHATt, Media Ownership Monitor Tunisie, 2016. http://tunisia.mom-rsf.org/fr/medias/

Z.A., "HAICA: pas de licence de diffusion audiovisuelle pour les hommes politiques», Kapitalis.com, 3 septembre 2014. http://www.kapitalis.com/ medias/24416-haica-pas-de-licence-dediffusion-audiovisuelle-pour-leshommes-politiques.html 


\title{
Chapitre 2
}

\section{Le Conseil national de la presse au Maroc: organe d'autorégulation de la profession de journaliste ou nouvel instrument de contrôle?}

\author{
Ahmed Hidass
}

Habité par la tradition et voulant incarner la modernité, le pouvoir marocain est paradoxal à plus d'un titre. En politique comme dans la communication et le journalisme, il oscille selon les périodes entre l'ouverture et son contraire. Le monopole public de l'audiovisuel et la prééminence de journaux officiels et partisans autorisés ont caractérisé l'espace médiatique pendant des décennies (Amar, Tuquoi, 2012 ; Boniface, 2011). Avec la mondialisation, la marchandisation croissante des productions culturelles, le développement des chaînes satellitaires, puis de I'Internet et de ses outils, du «journalisme citoyen » et de la consommation culturelle grand public, sa configuration a nécessairement changé.

Si les médias officiels, pratiquant un journalisme aseptisé, perdent de plus en plus de parts d'audience, tout comme la presse partisane, les médias étrangers ${ }^{1}$ et les sites alternatifs, sur le web et/ou diffusés par satellite, progressent et leur disputent la primauté. Pressés par le hirak² de février

\footnotetext{
${ }^{1}$ Les chiffres officiels font défaut, mais, au Maroc, près de $80 \%$ des ménages sont raccordés au réseau électrique et disposent de récepteurs de télévisions satellitaires. Selon l'Agence nationale de régulation des télécommunications (ANRT), $75 \%$ des ménages ont accès à internet. C'est ce qui permet aux déçus de l'offre télévisuelle marocaine de s'abonner à des bouquets de télévisions internationales, essentiellement auprès de serveurs IPTV informels.

2 Hirak: ce terme nouveau dans le vocabulaire politique arabe de la région MENA signifie un mouvement spontané de contestation populaire non encadré par les forces politiques conventionnelles (majorité et opposition représentées au parlement).
} 
2011 et craignant l'effet domino des "soulèvements arabes», les autorités marocaines ont anticipé l'escalade sociale ${ }^{3}$ et essayé d'encadrer, voire domestiquer, le phénomène.

Depuis I'Indépendance, le pouvoir marocain a procédé, à chaque révision/nouvelle constitution, à la création de nouveaux organes étatiques et à la modification de grands textes comme la loi sur les partis politiques, le mode de scrutin, la justice, le code des impôts, les langues officielles, l'espace médiatique, la configuration des régions, etc. Dans le discours officiel, il est question de refonder un «contrat social» et de réaliser des réformes visant à accompagner la «transition démocratique» du pays. Pourtant, selon Marina Ottaway (2011), il s'agit d'un changement dans la continuité, et, comme le remarque John Waterbury au début des années 1970, "on a souvent l'impression que le régime marocain n'a d'autre stratégie à long terme que d'espérer que ses tactiques de court terme continuent d'être payantes» (Waterbury, 1975 [1970], p. 180).

En 2011, la nouvelle Constitution reprend la liberté d'expression de façon plus ample. Expurgé des peines privatives de libertés, un nouveau Code de la presse est promulgué en 2016. Élargi à la presse électronique, il institue un organe ad hoc pour administrer la profession de journaliste: le Conseil national de la presse (CNP). Critiqué par les uns, loué par les autres, il est hybride en ce qu'il s'apparente à la fois à un ordre professionnel, à une autorité administrative et à un substitut du ministère de la Communication qui a été supprimé entre 2019 et 2021, la Communication devenant alors un département du ministère de la Culture, de la Jeunesse et des Sports.

En octobre 2021, la communication réapparaît dans un ministère de la Jeunesse, de la Culture et de la Communication. C'est ce qui explique la difficulté de caractériser le CNP d'un point de vue juridique, professionnel et institutionnel: est-ce une instance d'autorégulation du journalisme au Maroc et/ou un organe de sous-traitance administrative? Ce chapitre se propose de répondre à cette question en analysant la genèse de la constitution de cette instance et ses premières réalisations.

\footnotetext{
${ }^{3} 18800$ manifestations rien qu'en 2018-2019 et près de trois millions de citoyens dans la rue (chiffres donnés au début du mois d'octobre 2019 par Ahmed Chaouki Benyoub, délégué interministériel aux droits de l'homme aux Portes ouvertes de la Direction générale de la sûreté nationale à Tanger du 2 au 6 octobre 2019).
} 


\section{Le cadre constitutionnel, législatif et opérationnel des missions du CNP}

L'instauration du Conseil national de la presse doit être resituée, même brièvement, dans I'histoire contemporaine du cadre constitutionnel marocain. Depuis son indépendance, le système monarchique marocain a survécu à de grandes épreuves: deux tentatives de coup d'État, une transition monarchique sur fond d'«alternance politique problématique» (Enhaili, 1999) et des hirak à répétition. Du temps des médias dits traditionnels, les autorités contenaient les contestations et les neutralisaient, essentiellement via une gestion sécuritaire: investissement dans la répression, octroi de privilèges et harcèlement judiciaire des journalistes et écrivains critiques. Avec l'arrivée de l'Internet, des nouveaux médias et des réseaux sociaux, les mobilisations sociales et politiques ont gagné en visibilité à l'intérieur du pays comme à l'étranger. La réaction des autorités publiques est toujours de même nature, mais de plus en plus proactive.

\section{La nouvelle Constitution de 2011}

Dans le sillage des contestations populaires en Égypte, Syrie, Tunisie, Jordanie, Bahreïn et Libye, l'effet domino a fonctionné, touchant le Maroc (Bekkali, 2016) à travers l'émergence du Mouvement du 20 février 2011. Il se nourrit du même terreau que les précédents - chômage, inégalités sociales et territoriales, etc. - et s'insurge contre «le makhzen à la longévité insolente et aux rouages institutionnalisés» (Claisse, 1996). Les colères populaires sont désormais répétitives comme à Jerada (2018), dans le Rif (2017), à Zagora (2017), Sidi Ifni (2005-2008) ou Lafideq (2021). Général, populaire et pacifique, le Mouvement du 20 février 2011 a ébranlé les autorités marocaines par ses manifestations de masse dans toutes les villes du Maroc, l'obligeant à réagir. Rédigée par une commission désignée par le Roi, soumise à référendum avec une injonction officielle de voter en sa faveur, une nouvelle Constitution a été adoptée dès 2011 par $97,58 \%$ de votants "oui » contre 1,52\% de «non » et $0,90 \%$ de bulletins blancs ou nuls. Autrement dit, à quelques centièmes près, les votes furent les mêmes que pour les cinq Constitutions précédentes. À l'exception de l'Organisation de l'action démocratique populaire (OADP), un parti d'extrême-gauche, les partis politiques avaient tous voté «oui » en 1996 pour la précédente Constitution, estimant qu'elle répondait à leurs attentes et à la «bonne gouvernance» (El Ghazi, 2019). En 2011, ils ont tous soutenu la nouvelle Constitution. Contrairement aux précédentes, lacunaires en matière de communication et de médias, la 
nouvelle Constitution prévoit des dispositions pour l'encadrement dans ce domaine, tout en restant fidèle à l'organisation politique générale du pays.

\section{Nouvelle définition constitutionnelle de la liberté d'opinion et d'expression au Maroc}

Dans les cinq précédents textes constitutionnels (1962, 1970, 1972, 1992 et 1996), la liberté d'expression était définie par le même article 9. Demeuré inchangé de 1962 à 2011 et d'une économie sommaire, il définissait cette liberté de façon brève et restrictive. Avec la Constitution de 2011, un changement de forme est intervenu. Le dispositif consacré à la liberté de I'information est plus exhaustif: trois articles $(25,27$ et 28$)$ portent sur «la liberté d'opinion et d'expression». Pour autant, à l'exception d'une seule nouveauté, il s'agit de la même approche de la liberté de l'information qu'en 1962. La nouveauté est dans l'article 27, qui introduit le droit d'accès à l'information. Celui-ci est cependant soumis à des conditions et des exceptions importantes, telles que le fait que l'Office chérifien des phosphates est exempt de ce droit d'accès ou qu'il ne concerne pas la "société civile». Réservé légalement aux seules personnes physiques de nationalité marocaine, le formulaire de demande d'accès ("demande d'obtention» dans le texte en langue arabe) en témoigne. Comparé aux formulaires tunisien, américain ou finlandais, il est dissuasif et restrictif.

Le changement principal apporté par la nouvelle Constitution consiste dans le fait que la liberté d'opinion et d'expression n'est plus formulée au bénéfice du seul citoyen marocain, étant désormais un attribut général. Pour autant, les conditions de la participation des étrangers au capital de journaux, radios et télévisions au Maroc sont dissuasives vu que celle-ci est soumise à l'approbation des autorités (par exemple, un décret du Premier ministre pour la création d'un média par un étranger).

La nouvelle Constitution reconduit très largement l'ancienne acception de la liberté d'expression (Hidass, 2016) au sens où il s'agit toujours d'une liberté sous toutes ses formes (orale, écrite et artistique) et non par tous les moyens d'expression (presse écrite, radio, télévision, agence de presse, média en ligne, média hors ligne, etc.) comme le stipulent la Déclaration universelle des droits de l'homme et le Pacte international relatif aux droits civils et politiques auxquels le Maroc a souscrit. De même, la liberté d'opinion est consacrée de façon furtive. II n'est pas stipulé dans la Constitution que «nul ne peut être inquiété pour ses opinions», comme en droit international. Le "délit d'opinion», voire le "crime d'opinion», est toujours appliqué, comme 
le montre I'« affaire Ali Anouzla», directeur du média électronique Lakome. com, qui a été inculpé pour "apologie du terrorisme» en 2013 pour avoir indiqué un lien internet vers une vidéo d'Al-Qaïda au Maghreb islamique (Aqmi) diffusée sur le site du quotidien espagnol El Pais. Il en est de même pour les journalistes Hamid El Mahdaoui ${ }^{4}$, Taoufik Bouachrine ${ }^{5}$ ou Hajar Raissouni ${ }^{6}$ qui ont été inculpés pour des charges tout aussi lourdes. Les marges d'expression sont telles que certains ont préféré s'expatrier, comme par exemple Ahmed Reda Benchemsi, Aboubakr Jamai ou Ali Lmrabet"

Le régime de liberté d'expression et d'entreprendre prôné par la Constitution ne concerne essentiellement que la presse papier. En effet, elle est le principal secteur média ouvert à l'initiative privée non pas seulement partisane, ce qui n'est pas le cas de la télévision, qui relève, de fait, du monopole de l'État ${ }^{8}$. Ce secteur comme celui de la radio, ouvert plus largement au privé depuis 2006, est «régulé» par la Haute autorité de la communication audiovisuelle (HACA) créée par dahir (décret royal) et, depuis 2011, élevée au rang d'organisme constitutionnel. Celle-ci n'a jamais publié le plan de fréquences (occupées ou disponibles), et, depuis la première (2006) et deuxième (2009) vagues de licences, elle n'a pas lancé de nouveaux appels d'offres pour l'attribution de chaînes radio/TV. Les nouveaux médias privés

\footnotetext{
${ }^{4}$ Hamid El Mahdaoui, responsable du site badil.info et auteur de vidéos sur Youtube, a été condamné à trois ans de prison ferme assortis d'une amende de 30000 dirhams pour «non dénonciation d'un crime portant atteinte à la sûreté de l'État». Présent à El Hoceima, fief du hirak rifain, le journaliste aurait reçu des communications téléphoniques d'un Marocain résidant aux Pays-Bas, qui «laissait entendre tantôt qu'il finançait le hirak et son leader Nasser Zefzafi, tantôt qu'il avait des armes dans le coffre de sa voiture destinées aux manifestants d'Al Hoceima».

${ }^{5}$ Taoufik Bouachrine, directeur du quotidien de langue arabe Akhbar Al Yaoum, a été condamné à douze ans de prison pour "traite d'êtres humains", "abus de pouvoir à des fins sexuelles» et «viol et tentative de viol». Sa condamnation a été portée à quinze ans en appel et a été assortie d'amendes et de dédommagements pour les plaignantes.

${ }^{6}$ Hajar Raissouni, journaliste au quotidien Akhbar Al Yaoum, a été condamnée le 30 septembre 2019 à un an de prison ferme et deux années d'interdiction de l'exercice de la profession de journaliste pendant deux années supplémentaires pour «avortement illégal » et « débauche». Bénéficiant d'un large soutien à l'international, elle a été graciée le 16 octobre 2019 par le Roi Mohammed $\mathrm{VI}$ en dehors de toute fête religieuse ou nationale.

${ }^{7}$ Condamné en 2005 pour «diffamation», Ali Lmrabet a été interdit d'exercer la profession de journaliste pendant dix ans, à payer une amende de 50000 dirhams et à publier, à ses frais, le jugement le condamnant pendant trois semaines dans un quotidien arabophone.

${ }^{8}$ Ce monopole a été supprimé par le décret-loi du 10 septembre 2002 portant suppression du monopole d'État en matière de radiodiffusion et de télévision instauré par le dahir du 25 novembre 1924 relatif au monopole de l'État en matière de télégraphie et de téléphonie avec ou sans fil. Toutefois, depuis 2002, aucun appel d'offres, manifestation d'intérêt ou appel à candidatures n'a été lancé par la HACA pour la création de télévisions ou de radios privées.
} 
que compte le paysage audiovisuel marocain sont des radios conventionnées sur dossier (sans appel à candidatures) et des télévisions périphériques domiciliées à l'étranger, émettant vers le Maroc comme Télé Maroc, Chada FM ou Télé Découverte ou diffusées uniquement sur internet ${ }^{9}$.

Enfin, pour la première fois, la Constitution marocaine interdit la censure a priori, mais elle ne prévoit rien au sujet de la censure a posteriori, qui est plus dommageable en raison des pertes qu'elle occasionne à la censure d'un journal ou d'un livre après engagement des frais de production, d'impression et de distribution. La nouvelle Constitution n'évoque pas non plus internet. Pourtant, comme le rappelle le sommet NET Mundial au Brésil en 2014, ce secteur a généré ou renforcé des notions fondamentales comme le droit de connexion, le droit à la mort numérique, la non-discrimination, le droit à l'oubli numérique, la datacratie, etc. Préparée dans la précipitation, sous pression dudit «printemps arabe» dont il s'agissait d'éviter l'effet domino, la Constitution de 2011 est donc restée dans la lignée de la gouvernance générale du pays (Bendourou, 2012).

\section{Médias et culture au Maroc: un écosystème en difficulté}

Par-delà cette configuration constitutionnelle, c'est aussi la place de la production culturelle au Maroc qu'il faut rappeler rapidement pour mieux saisir les enjeux liés à la création du Conseil national de la presse. À quelques exceptions près comme le festival de musique Mawazine (Rabat), le Salon du cheval (El Jadida), le Festival de musique Gnaoua (Essaouira) et le festival des musiques sacrées du monde (Fès), qui bénéficient du sponsoring de grandes entreprises nationales, le secteur de la culture (livre, cinéma, théâtre, musique, spectacles sur scène, bibliothèques, arts plastiques, musées, sites et immeubles historiques, festivals populaires, etc.) est atrophié au Maroc. Avec ses 36 millions d'habitants en 2018, le pays compte à peine 27 salles de cinéma (1,57 million d'entrées en 2018), un tirage quotidien des journaux de moins de 150000 exemplaires (moins de 10 journaux pour 1000 habitants). Sur les 488 titres de la presse papier marocaine, 346 sont en arabe, 93 en français et 32 en arabe et en français, aucun titre n'existant en langue amazighe (ministère de la Communication, 2018). II en est de même des 7 stations de radio publiques et des 18 privées, puisqu'une seule d'entre elles propose un programme en langue amazighe. Pour la télévision, une seule chaîne est diffusée dans

\footnotetext{
${ }^{9}$ Le 25 mai 2021, Othmane El Firdaous, ministre de la Culture en charge du département de la Communication, a décidé d'étendre à $100 \%$ le contrôle du holding public audiovisuel SNRT aux chaînes de télévision $2 M$ et Medi1 TV, à la radio privée Medi1 et à la régie publicitaire Régie 3.
} 
cette langue sur les 7 chaînes du bouquet public proposé par la Société nationale de radiodiffusion et de télévision (SNRT) ${ }^{10}$. Pourtant les locuteurs de l'amazigh, langue officielle depuis 2011, sont majoritaires dans plusieurs bassins géographiques du pays. L'industrie du livre propose de 4000 à 6000 titres seulement (y compris les magazines et le livre scolaire) enregistrés au dépôt légal en 2018 (contre 81263 en France, 66890 en Turquie et 71548 en Allemagne) (International Publishers Association, 2020). En moyenne, les Marocains consacrent moins de deux minutes à la lecture par jour (HCP, 2020, p. 85) et fréquentent peu les rares bibliothèques et musées.

Copiée sur la France (Berteau, 2019; Bougon, 2019) où, sous contrôle du Parlement et de la Cour des comptes, les médias sont soutenus ${ }^{11}$ pour l'innovation, le pluralisme et la diversité (Fonds stratégique pour le développement de la presse, 2016), I'aide publique est problématique au Maroc (Rapport du ministère de la Communication, 2014). Sans texte de cadrage, elle a été instituée par l'État et n'a pas été auditée depuis l'indépendance du pays en 1956. Constitutionnellement dédiée à «l'organisation du secteur de la presse de manière indépendante et sur des bases démocratiques » et à la mise à niveau des entreprises médias, elle est sans levier ad hoc. Le modèle économique de cette aide et sa répartition inégale n'ont pas produit les résultats officiellement escomptés. Par conséquent, l'érosion de l'audience des médias nationaux est continue: la presse papier représente moins de 10 exemplaires pour 1000 habitants contre plus de 500 en Finlande, selon un rapport daté de 2019 (WAN/IFRA, 2019). Le paysage de la presse papier demeure atrophié et la liberté d'expression fortement encadrée par des «lignes rouges» (Marchetti, Benchenna, 2019).

\section{Le Conseil national de la presse, garant de la déontologie professionnelle, du pluralisme et de la diversité?}

La Constitution de 2011 a reconduit ou instauré 23 organes constitutionnels, dont la plupart sont nouveaux. Qu'ils soient à vocation consultative comme le Conseil national des droits de l'homme, prospective comme le Conseil de la

\footnotetext{
${ }^{10}$ Voir le site de l'autorité de régulation de l'audiovisuel, la HACA: https://www.haca.ma ${ }^{11}$ À l'exception de quelques titres de la presse papier (Le Canard enchaîné, L'Humanité, etc.), en ligne (Mediapart) et de quelques radios locales, les médias traditionnels français appartiennent tous à de grands groupes industriels et financiers. La légitimité des subventions publiques (Mauduit, 2020) pour ces médias est de plus en plus remise en question.
} 
concurrence ou décisionnelle comme la HACA, leurs membres sont cooptés par le pouvoir. Leurs attributions concernent beaucoup de domaines et débordent ou recoupent, de façon conflictuelle, les pouvoirs du parlement.

La communication, les médias, la culture et les langues du pays sont concernés par deux institutions: le Conseil national des langues et de la culture marocaine (CNLCM) et le Conseil national de la presse (CNP). Organisme constitutionnel et objet d'un projet de loi organique toujours en cours, le CNLCM aura à proposer les orientations stratégiques de l'État en matière de politique linguistique, médiatique et culturelle. De moindre importance dans la hiérarchie institutionnelle et objet d'un texte de loi, le CNP est par contre installé depuis le 5 octobre 2018. Hybride à la fois dans sa composition, ses pouvoirs et son financement, comparativement aux organismes similaires, sa mission et ses objectifs suscitent des interrogations. Est-il un organe d'autorégulation de la presse, un garant/gardien de la liberté d'expression, un substitut du ministère de la Communication ou un outil de plus au service du pouvoir?

\section{Les conseils de presse: des organes communs à des régimes politiques différents}

Le premier Conseil de presse a vu le jour en Suède en 1916. Depuis, ce genre d'institution s'est généralisé dans de nombreux pays. Le droit et la tradition reconnaissent à la presse des privilèges qui la placent au rang d'institution fondamentale. Elle les exerce au nom des citoyens en vertu d'une délégation supposée implicite, ce qui pose un problème puisque les journalistes et les médias ne sont pas élus. Par conséquent, pour conserver cette présumée délégation, la presse se doit de la mériter par une auto ou co-régulation, ce qui contribuerait à en faire un "quatrième pouvoir » légitime. De 1953, date de la création du Press Council du Royaume-Uni et jusqu'au scandale du piratage téléphonique ${ }^{12}$ du News of the World du magnat des médias Rupert Murdoch en 2011, l'expérience britannique passait pour un modèle universel. S'appuyant sur les rapports d'enquête de deux commissions royales (David Ross Commission, 1949, et Shawcross

\footnotetext{
12 En 2012, la Commission parlementaire des médias en Grande-Bretagne a révélé que 305 journalistes d'une trentaine de titres pratiquaient le piratage de téléphones (haking), l'usurpation d'identité (blagging) et la corruption pour obtenir des informations privées ou confidentielles. Ces «pratiques journalistiques» douteuses étaient routinières chez les tabloïds. Elles ont entamé le crédit du modèle de régulation britannique et conduit à repenser la déontologie journalistique ainsi que la gouvernance des médias en Grande-Bretagne.
} 
Commission, 1963) et créé par les professionnels eux-mêmes (propriétaires, rédacteurs en chef et journalistes), le Press Council présentait un caractère plutôt corporatiste (Hamon, 1977). Il en allait de même avec la Press Complaints Commission, créée en 1995 pour remédier aux défaillances de celui-ci et tenter de juguler ou, du moins tempérer, les pratiques agressives, déloyales et inquisitoires de la presse populaire britannique. En 2014, I'Independent Press Standards Organisation a été créée pour refonder un système de co-régulation dans lequel l'État joue un petit rôle (Hulin, 2015).

Depuis ces scandales et échecs à répétition au Royaume-Uni, c'est le Conseil de presse du Québec au Canada qui fait désormais école. Créé par les trois acteurs de l'information (les entreprises de médias, les journalistes et le public), il fonctionne comme un tribunal d'honneur et passe pour un modèle dans les démocraties matures et auprès de I'UNESCO. De même, la Tunisie attire également l'attention. Son modèle novateur propose la corégulation la plus collégiale. Le nouveau Conseil de presse créé en 2017 et mis en place en 2019 se distingue par une composition plus large. En plus des représentants des directeurs de journaux, des journalistes, du public (représenté par la Ligue tunisienne des droits de l'homme), il fait place aux institutions de presse ${ }^{13}$. L'ONG Article 19 considère ce conseil tunisien comme l'instance auto-constituée d'autorégulation/co-régulation de la presse la plus indépendante en Afrique et dans la région MENA.

Dans des pays où la presse est plus forte et développée, comme l'Espagne et les États-Unis, les journalistes ne disposent pas de conseil dédié à l'autorégulation de la profession et à la promotion des pratiques éthiques au sein de l'industrie de l'information. Mais ce n'est pas pour autant que la presse y soit plus ou moins éthique que dans des pays comme l'Allemagne, le Royaume-Uni, le Japon ou l'Australie. Les affaires de presse, comme les affaires civiles, sont justiciables devant les tribunaux de droit commun sans recours préalable à un quelconque organe de médiation.

\section{Un organe hybride ou une "exception marocaine "}

Pour l'Alliance des conseils de presse indépendants en Europe ${ }^{14}$ (AIPCE: 35 membres européens et 13 membres extra-européens associés), les conseils de presse (press councils) ou conseils de médias (media councils) sont des organes associatifs qui ont deux fonctions essentielles: la rédaction et

\footnotetext{
${ }^{13}$ Sur ce point, voir le chapitre 1 de cet ouvrage rédigé par Larbi Chouikha.

${ }^{14}$ Voir http://www.alliance-journalistes.net/rubrique60.html.
} 
la gestion d'un code de déontologie professionnelle (code of practices) et l'instruction des plaintes du public contre le contenu éditorial litigieux des médias; la défense de la liberté de l'information. Ces instances, constituées de journalistes et de représentants du public, peuvent être saisies et se saisir elles-mêmes des «dérapages» (atteinte à la vie privée, publi-rédactionnel déguisé, fake news, etc.), autant pour les dénoncer que pour les prévenir. Elles ne sont ni des ordres professionnels ni des instances judiciaires, mais des observatoires qui rendent publics et à intervalles réguliers des avis et rapports ${ }^{15}$.

Les conseils de presse doivent être indépendants du gouvernement. Ils sont auto-constitués et autofinancés par les membres sans subventions publiques. Dans les démocraties établies, ils se composent de représentants des journalistes, des éditeurs de presse et du public comme le Conseil de déontologie journalistique et de médiation créé en 2019 en France. Les codes de déontologie sont établis par les conseils eux-mêmes. Ils comprennent des principes éthiques propres aux médias, le journalisme et le public et ne se confondent pas avec le droit commun. L'objectif est d'améliorer la qualité de l'information (Hullin, 2015). Les conseils sont investis d'une responsabilité sociale qui permet au public de leur adresser ses plaintes sans assistance judiciaire obligée et gratuitement.

Au vu de ce descriptif des fonctions, composition et procédure de plainte auprès des conseils de presse tels que l'AIPCE les recommande à ses membres, qu'en est-il du Conseil national de la presse du Maroc? Le CNP est différent de ses homologues de I'AIPCE. C'est une structure publique et non associative comme en France, au Canada ou en Tunisie. Son local est mis à sa disposition par l'État. S'il est administré par des professionnels, nominalement privés, avec des compétences parapubliques, ses décisions sont d'ordre réglementaire: selon Khalid Cherkaoui Semmouni, le conseiller du ministre de la Culture, porte-parole du gouvernement en charge de la culture et l'information, "elles régissent le travail des journalistes du secteur privé et ne concernent pas les journalistes du secteur public » comme l'agence Maghreb Arabe Presse (MAP) ou le pôle public audiovisuel, la SNRT (MAP, 2020). Ces journalistes sont soumis au statut et au règlement intérieur de leur propre établissement. Conçu par le ministère de la Communication dans le cadre du nouveau Code de la presse et de l'édition, créé par un texte

\footnotetext{
15 On retrouve le même esprit et objectifs chez des corps de métier voisins comme les professionnels des relations publiques. Cf. le Code européen de déontologie professionnelle des relations publiques (Code de Lisbonne, 16 avril 1978, révisé le 13 mai 1989).
} 
de loi et promulgué par dahir, doté d'un règlement intérieur ${ }^{16}$ par décret du Premier ministre, il est subventionné par l'État avec des émoluments fixés aussi par décret de la Primature. L'article 4 de la loi 90-13 du 7 avril 2016 fixe la composition du CNP comme suit: un président, qui doit disposer d'une expérience dans le domaine de la presse de quinze ans au moins et n'ayant pas fait l'objet de mesures disciplinaires ou de jugements d'un tribunal (ce qui exclut les journalistes critiques); sept membres élus sur une liste bloquée parmi les journalistes professionnels; sept membres élus, individuellement, parmi les éditeurs de presse; sept membres représentant des institutions étatiques, associations et deux professionnelles honoraires. Un commissaire du gouvernement est désigné par l'État pour assister, à titre consultatif, aux réunions du Conseil et pour assurer la coordination entre ce dernier et I'Administration.

Doté d'un code de déontologie, il est officiellement rédigé par le Conseil national de la presse lui-même. Son préambule stipule qu'il est «élevé par le législateur au rang de texte de droit à force juridique obligatoire pour l'application effective des règles de déontologie professionnelle», ce qui tranche avec la valeur usuelle des codes d'éthique journalistique dans le monde. Si nul n'est censé violer une règle de droit sous peine de poursuites légales, une règle d'éthique peut être transgressée sans encourir d'autre sanction que la réprobation du public et des confrères.

Autrement dit, le Code de déontologie professionnelle est publié au Bulletin officiel du Royaume du Maroc comme un texte de loi ${ }^{17}$. Le CNP délivre la carte de presse aux journalistes du secteur privé. Véritable sésame pour l'exercice légal de la profession, les conditions d'obtention excluent le journalisme citoyen. D'ailleurs, pour produire des vidéo-amateur et les poster sur son propre blog, Youtube ou sur les autres réseaux sociaux, la démarche ne nécessite pas moins de cinq autorisations administratives ${ }^{18}$. Les décisions

\footnotetext{
${ }^{16}$ Décret du Premier ministre (11 mai 2020) portant Règlement intérieur du CNP. II fixe les règles d'organisation du CNP, les modalités d'exercice de ses attributions, les procédures de réception des plaintes et de traitement des affaires de déontologie et arrête la rémunération des différentes composantes du Conseil.

17 Décision du président du Conseil national de la presse portant publication du Code de déontologie professionnelle (BORM, 2019).

${ }^{18}$ Pour travailler dans la légalité, un créateur de contenu audiovisuel se doit d'avoir, selon les cas, plusieurs autorisations: le récépissé définitif de dépôt du dossier de presse électronique auprès du Parquet pour la création d'un site web, le récépissé définitif du dépôt de déclaration auprès de l'autorité locale si le créateur de contenu agit au nom d'une ONG, la carte de journaliste professionnel pour couvrir des événements, une autorisation du Centre cinématographique marocain pour les tournages et prises photographiques (délivrée aux entreprises uniquement, décret du 8 septembre 2021), une autorisation de l'Office des changes pour acquérir du matériel
} 
disciplinaires du CNP sont susceptibles de recours devant les tribunaux administratifs, ce qui fait du CNP un organe para-administratif (article 52 de la loi 90-13), mais il n'existe pas de voie de recours administratif ou judiciaire contre le refus d'attribution de la carte de presse à un journaliste par le Conseil. Les règles du Code sont à caractère normatif et impératif et non pas des devoirs et objectifs professionnels, comme de coutume, dans les grands codes de déontologie. Pour le président du CNP (Moujahid, 2019), "c'est de quoi assainir la profession au Maroc, barrer la route aux intrus et asseoir une culture journalistique responsable ${ }^{19}$ ». Pour les journalistes indépendants, c'est l'épée de Damoclès: "Le Code pénal et la loi anti-terroriste sont plus cléments que les normes professionnelles du CNP», explique Aziz Idamine (Idamine, 2019).

\section{Un «kit» de survivalisme politique?}

Voulu par l'État depuis une vingtaine d'années et initié de façon participative 20 et par une proposition de loi par Mustapha El Khalfi, ministre de la Communication (2012-2017) et ancien journaliste, le Conseil national de la presse fait désormais partie du paysage médiatique au Maroc depuis 2016. Il y a cependant lieu de se demander s'il n'a pas été créé pour sous-traiter la politique de l'État (le ministère de la Communication a disparu le 9 octobre 2019) en matière de liberté d'expression pour lui éviter d'être la cible des critiques directes internationales. Si dorénavant il y a problème de diversité, de culture ou d'expression, l'État ne peut en être tenu pour responsable. Le secteur des médias est régulé par les professionnels eux-mêmes, et les journalistes sont jugés par leurs pairs. La carte de presse naguère octroyée par le ministère de la Communication est désormais du ressort du CNP.

professionnel à l'étranger, une autorisation du ministère de tutelle pour filmer des bâtiments publics (le ministère de la Culture pour un monument historique), une autorisation de l'autorité administrative locale dans le ressort duquel se déroule le tournage, une autorisation du ministère de l'Intérieur pour mener un sondage ou soumettre un questionnaire, l'autorisation du Bureau marocain du droit d'auteur pour exploiter le folklore marocain et autres œuvres, etc.

${ }^{19}$ Déclaration du président du CNP à l'inauguration du siège de celui-ci, le jeudi 25 juillet 2019: "I'Immunisation du métier requiert un encadrement de l'accès à cette profession, conformément aux dispositions juridiques et d'éthique et selon des critères scientifiques et contractuels à même de barrer la route aux intrus, surtout face à la prolifération des nouvelles technologies de l'information.»

${ }^{20}$ Comme pour les deux autres textes qui composent le nouveau Code de la presse, le ministère de la Communication, dans le cadre d'une démarche participative, a soumis, pour consultation, la proposition de loi sur le CNP aux départements ministériels concernés, la "société civile», les ONG nationales et internationales, le Syndicat national de la presse marocaine (SNPM), la Fédération marocaine des éditeurs de journaux (FMEJ), les partis politiques etc. Toutefois, ledit ministère n'a pas publié les commentaires reçus sur son site web. Ils sont restés confidentiels. 
Cependant, sa délivrance doit encore attendre un décret de l'exécutif, qui en fixera les modalités et conditions d'attribution sur la base de la loi relative au statut de journaliste professionnel ${ }^{21}$. Véritable sésame pour les quelque 3000 journalistes légalement en exercice au Maroc, la carte de presse est constitutive de droit et non pas déclarative de droit (Hidass, 2000).

\section{Le Conseil national de la presse à l'épreuve des faits et de ses missions}

Constitué en décembre 2019, c'est-à-dire bien après son homologue marocain, le Conseil de déontologie journalistique et de médiation (CDJM) en France a déjà réceptionné des saisines déposées par le public, rendu des avis, répondu à ses détracteurs et noué des partenariats nationaux et internationaux. Fixées par la loi, ses missions ont trait à la déontologie, la médiation et l'octroi de la carte de presse. Alors que l'on s'attendait à un état de la déontologie du journalisme au Maroc comme œuvre première et principale du CNP, celui-ci a réservé sa première sortie à des sujets comme les fake news et l'état de la presse papier au Maroc. Interpellé dernièrement par la «société civile» et des collectifs de journalistes marocains au sujet de la déontologie, il n'a pour l'instant pas réagi.

\section{Le Conseil national de la presse et les fake news}

La première sortie du Conseil national de la presse était en effet réservée aux fake news. À l'origine de la défiance actuelle du public envers les médias, casse-tête juridique pour les Nations Unies (Hidass, 2021), les informations fausses ou déformées sont un avatar de la communication en flux continu et de grande diffusion. Phénomène mondial sur fond de manipulation, de concurrence déloyale, de rivalités entre États, de désinformation, l'expression "fake news» a gagné en popularité durant le mandat du président américain Donald Trump (2017-2021), qui l'utilisait abondamment, décernant par exemple un prix ad hoc au New York Times et à la chaîne de télévision américaine CNN en 2018. Amplifiées par les social bots, profilés par le deep fake et l'intelligence artificielle, les fake news sont devenues un phénomène de société avec des effets de plus en plus préjudiciables sur les victimes.

\footnotetext{
${ }^{21}$ Loi $n^{\circ}$ 89-13 relative au statut de journaliste professionnel, 27 avril 2016 (promulguée par le dahir $\left.n^{\circ} 1-16-51\right)$.
} 
Pour combattre le phénomène, les réponses sont nombreuses, au niveau mondial, et se résument en deux volets, préventif et répressif. Au Maroc, le Code de la presse réprime les fausses nouvelles et prévoit un droit de réponse et de rectification. Un projet de loi adopté par le conseil de gouvernement le 19 mars 2020 vise à réglementer l'utilisation des réseaux sociaux, des réseaux de diffusion et des réseaux assimilés. Pour le volet préventif, alors que les grandes plateformes renforcent la modération de leur contenu et que des acteurs comme I'UNESCO proposent l'éducation aux médias (Ireton, Poseti, 2019), le CNP a entrepris, pendant le confinement de 2020 pour cause de pandémie Covid-19, une campagne de publicité tous médias, publics et privés. Celle-ci recommandait la lecture de «la presse professionnelle marocaine pour lutter contre le virus des fausses nouvelles» en présentant une femme en tenue traditionnelle des pays du Golfe lisant un journal de sa région.

\section{Grille de lecture du CNP pour l'état de la presse, les journalistes et la déontologie}

Son site web ${ }^{22}$ étant encore très récent, basique, et ses publications sommaires et formelles, il est difficile d'analyser son action. Pour autant, ses communications médiatiques sur l'état de la presse et ses silences sur des affaires de presse et de déontologie le concernant semblent montrer que sa grille de lecture ne semble pas différer de celle de l'ex-ministère de la Communication. En effet, à quelques différences près, les indicateurs retenus par le CNP recoupent entièrement ceux dudit ministère dans son rapport annuel de 2012 à $2017^{23}$. Nous sommes loin des indicateurs universels retenus par I'UNESCO comme cadre pour l'évaluation des médias: promouvoir la liberté de la presse et le pluralisme des médias, le développement des médias communautaires et le développement des ressources humaines (Unesco, 2010).

Alors que les missions du CNP se rapportent nominalement à la liberté de la presse, la déontologie, la formation professionnelle, la médiation et la carte de presse, le CNP a réservé sa première sortie médiatique à l'état de la presse papier marocaine. Monochrome, en perte d'audience depuis que les titres critiques successifs ont disparu, elle demeure en crise et

\footnotetext{
22 Voir https://cnp.press.ma (Consulté le 16 mai 2021).

${ }^{23}$ Le ministère de la Communication a publié un rapport annuel sur «les efforts pour la promotion de la liberté de la presse au Maroc» de 2012 à 2017.
} 
peut-être plus encore après avoir basculé vers le web et le format PDF. Subventionnée depuis toujours par l'État, la presse papier a vu son aide publique institutionnalisée par un décret du Premier ministre Abdellatif Filali, suite à un discours du Roi Hassan II en 1989. Généralisée à la presse papier et numérique, revue périodiquement à la hausse et soumise à un contrat-programme, elle permet aux entreprises de presse inscrites au registre de la Commission paritaire de presse écrite de survivre en partie, d'autant plus dans un contexte où les GAFAM captent l'essentiel des budgets publicitaires. Conformiste, limitée par les «lignes rouges», manquant de proximité et avec un personnel sous-qualifié, elle n'a pas de modèle économique viable.

Avec la pandémie de Covid-19 la situation a empiré. Par arrêté du ministère de la Culture, le 23 mars 2021, les éditeurs de magazines et journaux ont été appelés à suspendre la publication et la distribution de leurs supports papiers jusqu'à nouvel ordre. Le quotidien critique Akhbar Al Yaoum a cessé sa parution début 2021. Boycotté par des annonceurs publics et privés, ses ventes papier assuraient $90 \%$ des revenus du journal (Oudrhiri, 2020). C'est dans ce contexte que le CNP a rendu public, le 9 juillet 2020, son rapport sur l'état de la presse papier intitulé «les effets de la pandémie de Corona sur la presse marocaine» (Conseil national de la presse, 2020). Général, classique et expurgé des causes structurelles qui font la faiblesse de la presse marocaine, le rapport avait pour but de solliciter plus de soutien financier de l'État. Ainsi, sur demande de l'Association nationale des médias et des éditeurs (ANME) constituée pendant le confinement général, le 24 juin 2020, une enveloppe de 205 millions de dirhams a été débloquée par le gouvernement pour soutenir le secteur. En 2021, une autre enveloppe de 340 millions de dirhams a été allouée à 140 entreprises mais non sans protestation des bénéficiaires pour discrimination ${ }^{24}$.

Au-delà des besoins économiques, l'éthicisation du journalisme au Maroc est une priorité pour le président du Conseil national de la presse: «L'immunisation du métier requiert un encadrement de l'accès à cette profession, conformément aux dispositions juridiques et d'éthique et selon des critères scientifiques et contractuels à même de barrer la route aux intrus, surtout face à la prolifération des nouvelles technologies de

\footnotetext{
${ }^{24}$ Voir le communiqué (en arabe) de la Fédération marocaine des éditeurs de journaux dans Bayane Al Youm, 10 mai 2021, pages 1 et 16.
} 
I'information ${ }^{25}$.» Mais, depuis sa création, le CNP n'a pas encore établi de rapport sur l'éthique et la déontologie du journalisme au Maroc, alors que les controverses à ce sujet ne manquent pas de la part des médias partisans, privés et proches du champ du pouvoir.

À l'occasion de l'état d'urgence sanitaire décrété par le gouvernement depuis le 20 mars 2020, le confinement et le couvre-feu nocturne, les fake news, les thèses complotistes et la polémique au sujet des vaccins ont proliféré au Maroc comme ailleurs. C'est dans ce contexte que le CNP a fait un «rapport intérimaire au sujet de la déontologie pendant la pandémie de Covid-19» (CNP, 2020).

Réduit à quelques pages, anonymisé, ne citant aucun journaliste ou média de son nom et ne présentant aucun texte, photo ou vidéo litigieux à l'appui, le document pointe les manquements au respect du Code de déontologie et exhorte les journalistes à faire preuve de professionnalisme et de responsabilité dans le traitement des informations relatives à la pandémie. Toutefois, le rapport ne vise que les journalistes de la presse papier et en ligne. Comme s'ils ne relevaient pas de son ressort, il ignore les journalistes de l'agence de presse officielle MAP et des chaînes de radio et de télévision. De plus, sachant que la plupart des contenus médiatiques sur le coronavirus sont le produit de citoyens, youtubeurs, blogueurs et lanceurs d'alerte non reconnus par le CNP comme journalistes professionnels, le rapport demeure en décalage avec le paysage médiatique.

Alors que le Maroc était en passe d'entrer dans l'état d'urgence sanitaire suite à la propagation de la pandémie dans le pays, le ministre socialiste de la Justice Mohamed Benabdelkader a élaboré un projet de loi pour lutter contre les allégations mensongères et l'utilisation abusive des réseaux sociaux, des réseaux de diffusion et réseaux similaires. Présenté au conseil de gouvernement le 19 mars 2020, le texte a fuité et suscité l'indignation de la société civile. Baptisé «loi bavette» par les internautes marocains, il est question de criminaliser l'appel au boycott commercial (de plus en plus fréquent), la création et le partage de contenus sensibles pour le pouvoir sur les réseaux sociaux. Cette tendance liberticide semble mondiale. Sous prétexte de lutter contre le séparatisme et les discours haineux sur la Toile, les autocraties, les «démocraties défaillantes» et les réseaux sociaux (GAFAM) ont engagé des mesures, officiellement, pour

\footnotetext{
${ }^{25}$ Déclaration de Younes Moujahid, président du CNP, à l'inauguration de cette institution le 25 juillet 2019 à Rabat: https://Int.ma/conseil-national-de-presse-inaugure-nouveau-siegea-rabat/
} 
«modérer» les contenus médiatiques et, pratiquement, pour censurer les contenus politiquement sensibles. D'où la réaction du secrétaire général des Nations Unies pour dénoncer les pays qui, au nom de la Covid-19, entravent ou abolissent les libertés fondamentales.

Sur fond de réprobation générale et décrié sur les réseaux sociaux, le projet de loi a été pour l'instant stoppé par le gouvernement, mais le CNP n'a jamais pris de position à son sujet. La promotion de la liberté de la presse relève pourtant de ses missions, comme il le rappelle dans son communiqué du 15 novembre 2020 à l'occasion de la Journée nationale de l'information et de la communication. Il en est de même pour les faits de presse devant la justice marocaine.

\section{Les manifestes d'écrivains, artistes et journalistes marocains pour la liberté d'expression et contre "les médias de diffamation»}

Comme dans beaucoup de pays, il est fréquent qu'au Maroc des journalistes, des écrivains ou des penseurs soient «blacklistés» pour leurs opinions. C'était le cas de Fatima El Mernissi, Mohamed Khair-Eddine, Mohamed Choukri et Mehdi El Mandjra, très connus à l'international et ayant obtenu des distinctions honorifiques, mais interdits d'antenne dans leur pays. De même, à part une dizaine d'années d'ouverture (1995-2005) pour les ONG nationales ( $\mathrm{AMDH}$, etc.) et internationales (Human Rights Watch, etc.), les libertés publiques sont très contraintes. Ayant expurgé le nouveau Code de la presse et de l'édition de 2016 des peines privatives de liberté, une nouvelle ère journalistique semblait depuis s'annoncer. Les récentes condamnations de journalistes à des peines d'emprisonnement en vertu du Code pénal (Bobin, 2021) témoignent d'un changement dans la continuité. Auparavant, les plumes jugées critiques étaient condamnées pour dépassement des «lignes rouges», outrage aux institutions et aux corps constitués, diffamation, apologie du terrorisme, intelligence avec l'étranger, trouble à l'ordre public, financement étranger, etc. Depuis quelques années, des motifs supplémentaires liés à la vie privée ont fait leur apparition: avortement illégal, relations sexuelles hors mariage, viol et tentative de viol, traite d'êtres humains, abus de pouvoir à des fins sexuelles, drogue etc. Le CNP n'en fait pas état dans ses publications, jugeant probablement que ces condamnations sont sans lien avec l'exercice professionnel.

II n'a pas réagi non plus aux interpellations publiques de membres de la "société civile» au sujet de la déontologie du journalisme et des médias au Maroc. Le 16 juillet 2021, un collectif de 110 journalistes a en 
effet rendu public un "manifeste contre les médias de diffamation» (Le Desk, 2020) adressé aux autorités publiques, au ministère de tutelle, au Conseil national de la presse, aux organisations et structures syndicales de la presse et des médias au Maroc et au Groupement des annonceurs du Maroc. Pour le collectif, «à chaque fois que les autorités ont poursuivi une voix critique, certains journaux et sites web se sont empressés d'écrire des articles diffamatoires sans aucune éthique professionnelle, voire enfreignant les lois organisant la presse au Maroc ». De même, le 11 août 2021, un autre collectif de 400 artistes, écrivains et journalistes ont diffusé un manifeste dénommé «Cette ombre est là » (Le Desk, 2020) pour « dénoncer les médias de diffamation, les harcèlements et les emprisonnements de journalistes, les arrestations de citoyens.nes qui ont exprimé leurs opinions sur les réseaux sociaux et les violentes répressions des manifestations... ».

\section{Conclusion}

À part quelques échos en ligne au Maroc et à l'étranger, les manifestes des deux collectifs d'artistes, écrivains et journalistes marocains ont été ignorés par la presse traditionnelle écrite et audiovisuelle. Le CNP n'en a pas pris acte et ne s'est pas saisi des deux «affaires», même si elles relèvent de son domaine d'action. D'où la question: ce conseil est un organe d'autorégulation de la presse ou un outil de contrôle supplémentaire dans une configuration d'autoritarisme compétitif?

Depuis I'Indépendance, les autorités marocaines ont procédé, à chaque nouvelle constitution, à la création de nouveaux organes de l'État et à la modification de grands textes comme la loi sur les partis politiques, le mode de scrutin, la justice, le Code des impôts, la langue officielle, l'espace médiatique, la configuration des régions, etc. C'est paradoxalement (Vermeren, 2020) pour accompagner la «transition démocratique» du pays. Dans les faits, il s'agit davantage d'instruments pour la reproduction de l'ordre social et politique. Ainsi, la Constitution de 2011 a institué quelque 23 organes constitutionnels. La plupart d'entre entre eux sont en veille et consistent en des postes pour les grands commis de l'État. C'est le cas par exemple du Conseil national de l'enseignement. Alors que la pandémie de Covid-19 pose problème à l'Éducation nationale depuis 2020 (adaptation des cursus à la crise, cours en ligne, confinement, gestes barrières...), ledit conseil ne s'est pas exprimé. La crise est en effet gérée principalement par le ministère de l'Intérieur et, plus secondairement, par le ministère de l'Éducation nationale. Apparemment, la création de ces instances a pour 
objectif de fidéliser l'élite, multiplier les garde-fous contre d'éventuels soulèvements de plus grande ampleur et s'ajuster aux attentes des institutions internationales. L'espace public conventionnel étant fortement délimité et contraint, les institutions représentatives en hibernation, les appels d'offres et à candidatures profilées, etc., les discours de colère et d'opposition ont investi d'autres espaces que les médias stricto sensu, plus précisément des espaces alternatifs de protestation comme les stades de football et les réseaux sociaux. La longévité exceptionnelle du régime, de son discours, sa gouvernance, sa culture et son étiquette sont à nouveau mis l'épreuve par ces déplacements des contestations.

\section{Bibliographie}

AmAR Ali, TUQuol Jean-Pierre, Paris-Marrakech: luxe, pouvoir et réseaux, Paris, Calmann-Levy, 2012.

BeKKALI Abdeslam, L'An 1 de la cyberdémocratie au Maroc, Casablanca, Editions Hammouch, 2012.

BenCHENNA Abdelfettah, MARCHETtI Dominique, "Écrire entre les "lignes rouges": I'espace de la presse électronique au Maroc et ses enjeux", in Péquignot Bruno (dir.), Les Industries culturelles et créatives dans la mondialisation, Paris, L'Harmattan, 2019. https://hal.archives-ouvertes.fr/ hal-02109002/.

BenChenNA Abdelfettah, KsIKES Driss et MARChETtI Dominique, "La presse au Maroc : une économie très politique: le cas des supports papier et électronique depuis le début des années 1990 », Questions de communication, $\mathrm{n}^{\circ} 32$, 2017. https://doi.org/10.4000/questionsdecommunication.11527

Boniface Pascal, Les Intellectuels faussaires, Paris, Éditions Jean-Claude Gawsewitch, 2011.

CLAISSE Alain, «Le Makhzen aujourd'hui », in Le Maroc actuel: une modernisation au miroir de la tradition, sous la dir. de Jean-Claude Santucci, Aix-enProvence, IREMAM, coll. Connaissance du monde arabe, 2013 [1992]. DOI: https://doi.org/10.4000/books.iremam.2431

COLLECTIF, Maroc: la guerre des langues, Casablanca, Éditions Toutes Lettres, coll. Questions, 2018.

Crouzet Thierry, Le Cinquième pouvoir, Paris, Bourin Editeur, 2007.

GaNz Pierre, Chroniques de déontologie, Paris, Riveneuve-PUF, coll. Journalisme aujourd'hui, 2018. 
HAMON Francis, «Liberté et responsabilité de la presse en Grande-Bretagne», Notes et études documentaires, nos 4448-4449-4450, 1977.

HIDASS Ahmed, "Le statut de journaliste professionnel au Maroc», in Cahiers du journalisme, $\mathrm{n}^{\circ} 8,2000$. https://www.cahiersdujournalisme.net/ pdf/08/13_Hidass.pdf

HIDASS Ahmed, "Quand l'exception confirme la règle: I'encadrement juridique de la liberté de la presse écrite au Maroc», L'Année du Maghreb, n 15, 2016. DOI: https://doi.org/10.4000/anneemaghreb.2774.

HIDASS Ahmed, «Fake news et le droit international: un siècle d'approches juridiques par la Société des Nations et l'Organisation des Nations Unies», in Déontologie de l'information: manipulation des médias et Post truth politics dans un monde instable, sous la dir. de M. Kirat, Doha, Publications de I'Université du Qatar, Département de la Communication, 2021 (en arabe).

HULLIN Adeline, Auto-régulation et liberté des médias en Europe: impact, perspectives et limites, Editions Panthéon-Assas, coll. Thèses, 2015.

HUNT David James Fletsher (baron Hunt), «Press Regulation», Politics.co.uk, March 2012. http://www.politics.co.uk/reference/press-regulation

IRETON Cherilyn, Posetti Julie, Journalisme, "fake news» et désinformation: manuel pour l'enseignement et la formation en matière de journalisme, Paris, UNESCO, série de I'UNESCO sur l'enseignement du journalisme, 2019. https://fr.unesco.org/fightfakenews [consulté le 21 mai 2021].

LEVITSKY Steven, Competitive Authoritarianism: Hybrid Regimes After the Cold War, London, Cambridge University Press, 2011.

MATTELART Armand, Diversité culturelle et mondialisation, Paris, La Découverte, coll. Repères, 2017.

NHAILI Aziz, "L'alternance politique verrouillée», Confluences Méditerranée, $\mathrm{n}^{\circ} 31$, automne 1999. https://iremmo.org/wp-content/uploads/2016/02/3105. enhaili.pdf.

OtTAWAY Marina, «The New Moroccan Constitution: Real Change or More of the Same?», 2011. https://carnegieendowment.org/2011/06/20/newmoroccan-constitution-real-change-or-more-of-same-pub-44731

RAMPAL Kuldip R., "The concept of Press Council», International Communication Gazette, n 2, n 28, 1981. DOI: https://doi.org/10.1177\% 2F001654928102800202

SefRIOUI Kenza, La Revue Souffles, 1966-1973: espoirs de révolution culturelle au Maroc, Casablanca, Éditions du Sirocco, 2012. 
SIRINELLI Marie, Auto-régulation de l'information : comment incarner la déontologie? Rapport remis à $\mathrm{M}^{\text {me }}$ Aurélie Filipetti, ministre de la Culture et de la Communication, Paris, 13 février 2014. https://www.culture.gouv. $\mathrm{fr} /$ Espace-documentation/Rapports/Autoregulation-de-I-informationComment-incarner-la-deontologie

TouzAnı Amina, La Politique culturelle au Maroc, Casablanca, Editions La Croisée des chemins, 2017.

VeRMEREN Pierre, Le Maroc en 100 questions: le royaume des paradoxes, Paris, Editions Tallandier, 2020.

WATERBURY John, Le Commandeur des croyants: la monarchie marocaine et son élite, Paris, Editions PUF, 1975 [édition anglaise, 1970].

\section{Sources}

AGENCE FRANCE PRESSE, "Au Maroc, 110 journalistes se mobilisent contre la presse de diffamation », Le Monde, 16 juin 2020.

Al OUSBOUE SAHAFI (la rédaction), «L'État va-t-il revoir la question... des marabouts et zaouïas?», n 1405/968, 22 février 2018.

BELLANGER Anthony, "Quel recours contre les dérapages médiatiques", Le Monde diplomatique, décembre 2018.

Bellanger Anthony, "En France, un projet controversé », Le Monde diplomatique, décembre 2018.

BERTEAU Alexandre, "Les éditeurs de journaux demandent un soutien de l'État », Le Monde, 11 avril 2019.

BoBIN Frédéric, "Au Maroc, la répression contre la presse se durcit », Le Monde, 17 avril 2021.

Bougon François, «Le gouvernement va réformer la distribution de la presse», Le Monde, 11 avril 2019.

BRISSET Claire, "Le long cheminement vers la dignité », Le Monde diplomatique, décembre 2018.

BULLETIN OfFICIEL DU ROYAUME DU MAROC (BORM), nº 6799 du 29 juillet 2019.

LE MONDE DIPLOMATIQUE (la rédaction), «Ce rapport qui accable les médias britanniques», janvier 2013.

CONSEIL NATIONAL DE LA PRESSE (CNP), Les Effets du coronavirus sur la presse et les mesures de sortie de crise, Rabat, Rapport du CNP, mai-juin 2020 (en arabe). https://cnp.press.ma/1334-2/ 
DESK (le) (la rédaction), «Manifeste: 110 journalistes marocains dénoncent la prolifération des médias de diffamation », 16 juillet 2020. https://ledesk. ma/2020/07/16/110-journalistes-marocains-denoncent-la-proliferationde-medias-de-diffamation

DESK (le) (la rédaction), «Manifeste: 400 articles et acteurs culturels marocains dénoncent la répression policière au Maroc», 11 août 2020. https://ledesk. $\mathrm{ma} /$ encontinu/400-artistes-et-acteurs-culturels-denoncent-la-repressionpoliciere-au-maroc

DILAMI Abdelmounaim, «A quoi va servir le Conseil national de la presse?», Union Internationale de la presse francophone, 6 juillet 2018. https:// www.presse-francophone.org/fr/generalites/article/maroc-a-quoi-vaservir-le-conseil-national-de-la-presse

HammouDI Ismail, Fadl Allah Siham, Rissouni Hajar, Belachgar Abdelhak, Karmoussi Adil, "Nouveau cycle dans la guerre identitaire», Akhbar Al Youm, 5-6 janvier 2018 (en arabe).

HAUT-COMmISSARIAT AU PLAN (HCP), Direction de la statistique, Les Indicateurs sociaux du Maroc, Rabat, Editions du HCP, 2020.

HIZAOU Abdelkrim, "Deux conseils de presse, en Tunisie et au Maroc», Observatoire arabe du journalisme, 8 septembre 2016. https://ajo-fr.org/ derniers-articles/deux-conseils-de-presse-tunisie-maroc.

IDAMINE Aziz, "Le Code de déontologie: code de répression de la presse», Akhbar Al Youm, 6 août 2019 (en arabe).

MAGHREB ARABE PRESS (MAP), "Les décisions du CNP sont d'ordre réglementaire et ne concernent pas les journalistes du secteur public, dont la MAP», 25 mars 2020. http://www.mapexpress.ma/actualite/activitegouvernementale/les-decisions-du-cnp-dordre-reglementaireconcernent-pas-les-journalistes-du-secteur-public-dont-map/

MALER Henri, «Un conseil de la presse? À quelles conditions et comment?», 11 décembre 2018. https://www.acrimed.org/Un-Conseil-de-la-PresseA-quelles-conditions-et

MAUDUIT Laurent, «Les milliardaires qui possèdent la presse plument l'État», Mediapart, 9 juillet 2020. https://www.mediapart.fr/journal/france/080720/ les-milliardaires-qui-possedent-la-presse-plument-I-etat?onglet=full

Monvalon (de) Jean-Baptiste, «Une haine qui vient de loin: aux sources d'une mauvaise réputation », Le Monde, cahiers Medias et Pixels: Idées, 26 janvier 2018. 
MouJAHID Younes, «Encadrer l'accès à la profession en vue de barrer la route aux intrus», Libération, 29 juillet 2019. https://www.libe.ma/YounesMoujahid-Encadrer-l-acces-a-la-profession-en-vue-de-barrer-la-routeaux-intrus_a110517.html

ORGANISATION DE LA SÉCURITÉ ET DE LA COOPÉRATION EN EUROPE, Le Guide pratique de l'auto-régulation des médias, Vienne, Publications OSCE, Bureau du représentant de la liberté des médias, 2008. https://www.osce.org/files/f/ documents/3/f/31498.pdf

OUDRHIRI Kawtar, "La presse prise à la gorge par le coronavirus», Tel Quel, $n^{\circ}$ 895, 20-26 mars 2020. https://telquel.ma/2020/03/25/la-vague-decoronavirus-met-en-peril-le-secteur-de-la-presse_1675500

UNESCO, Programme intergouvernemental pour le développement de la communication (PIDC), Indicateurs de développement des médias: cadre pour l'évaluation du développement des médias, Paris, UNESCO, 2010. https://fr.unesco.org/programme/ipdc/initiatives/mdis

WAN/IFRA, World Press Trends, 2019. https://www.wan-ifra.org/reports/2019/10/28/ world-press-trends-2019 



\title{
Chapitre 3 \\ La régulation très contrôlée du champ médiatique en Algérie
}

\author{
Chérif Dris
}

Pour contrecarrer toute tentative d'exporter les vagues de révoltes qui ont secoué certains pays de la région (Libye, Tunisie, Égypte et Syrie), les autorités politiques algériennes ont adopté une série de mesures destinées à desserrer l'étau qui tenaille la "société civile» et les médias. C'est ainsi que de nouvelles lois sur les partis, les associations et les médias ont été adoptées dans le cadre des réformes annoncées le 15 avril 2011 par le président Abdelaziz Bouteflika.

L'une des premières conséquences de ces ouvertures relatives autorisées au sommet de l'État est l'émergence de chaînes de télévision privées cette année-là, en l'absence même de tout cadre juridique autorisant leur création. En 2012, une loi organique sur l'information a codifié davantage le champ des médias, ce dispositif juridique étant conforté en mars 2014 par la loi sur l'audiovisuel et, en août 2016, par des décrets d'application définissant les conditions de création de chaînes de télévision et de radios privées.

Si la nouvelle configuration juridique a permis la pluralité des supports médiatiques et brisé le monopole de l'État sur l'audiovisuel, l'évolution la plus notable reste le développement de la presse en ligne, qui vient disputer aux médias traditionnels la primauté d'accès aux sources d'information et de diffusion des nouvelles. Pour autant, cette émergence de la presse électronique est freinée par les ambiguïtés juridiques contenues dans la loi organique de 2012. À cela s'ajoute un contexte économique très morose avec ses effets négatifs sur tous les supports de la presse algérienne.

En arrière-plan de ces mutations du système médiatique se pose la question de sa régulation. La loi organique de 2012 institue deux 
autorités dans ce domaine: une pour l'audiovisuel, l'autre pour la presse écrite. Si la première a été créée en 2014, adossée à un texte de loi régissant ce secteur, celle régulant la presse écrite tarde à voir le jour. C'est la régulation des médias algériens dans toute leur diversité qui est l'objet central de ce chapitre, où il sera question non seulement du cadre juridique et institutionnel régissant cette activité mais aussi du mode de régulation adopté en la matière. Toutefois, on ne saurait étudier les politiques de régulation en les dissociant de leur contexte politique. En effet, les transformations de l'espace médiatique algérien sont aussi la conséquence de reconfigurations politiques destinées à permettre au régime de se reproduire. C'est pourquoi il faut soulever les questions suivantes. Comment peut-on qualifier le mode de régulation adopté? Dans un contexte marqué par un interventionnisme accru de l'État dans les sphères politique, économique et culturelle, les dispositifs de régulation mis en place contribuent-ils à remettre de l'ordre dans un champ dérégulé? Ce discours sur la régulation a-t-il trouvé sa matérialisation sur le terrain? Quelles sont les contraintes qui se dressent devant ces instances de régulation dans l'accomplissement de leur mission?

Pour répondre à cette série d'interrogations, nous avons tout d'abord travaillé sur le socle juridique et législatif encadrant le champ médiatique algérien. Nous avons procédé à une analyse des textes promulgués depuis la loi organique sur l'information (janvier 2012) : la loi relative à l'audiovisuel (2014), les décrets d'application portant sur la création de chaînes de télévision et de radios privées (2016) et, enfin, le décret d'application encadrant la presse électronique (2020).

Cette analyse documentaire est complétée par des entretiens semidirectifs avec les différents acteurs du champ médiatique, privés et publics, et tous supports confondus. Ces entretiens sont utilisés pour fournir les éléments d'information que les textes de lois ne peuvent nous apporter, mais aussi pour restituer les positions de ces acteurs par rapport aux textes de lois promulgués et au travail accompli jusqu'ici par l'autorité de régulation de l'audiovisuel. En raison de la situation sanitaire depuis mars 2020, il ne nous a pas été facile de réaliser des entretiens. La plupart des acteurs que nous avions contactés ont opposé une fin de non-recevoir à nos demandes, tandis que d'autres ont avancé une multitude de prétextes pour se dérober. Pour ceux qui ont accepté, nous étions obligés, pour certains d'entre eux, de nous contenter d'entretiens par téléphone ou via la technique de visioconférence (Messenger notamment). 
Dans ce chapitre, il s'agit en premier lieu de restituer l'espace médiatique algérien à travers ses acteurs et ses structures. En deuxième lieu, l'analyse portera sur le dispositif légal et institutionnel encadrant cet univers et, notamment, les mécanismes de régulation. Enfin, cette régulation sera étudiée à travers les pratiques et les contraintes qui se dressent devant elle.

\section{Le champ médiatique algérien : acteurs et structures}

Après trois décennies de cloisonnement, le champ médiatique algérien s'est ouvert et diversifié. La Constitution de 1989 instituant le multipartisme et le pluralisme médiatique avait permis l'éclosion de dizaines d'organes de presse privés, rompant ainsi avec une ère de monopole et d'unanimisme dans ses différentes expressions. Cette ouverture a été cependant limitée à la presse écrite, le secteur audiovisuel demeurant sous l'emprise de l'État. La promulgation du code de l'information de 1990 a signifié la dérégulation de ce secteur et son insertion dans la logique du marché. En théorie, l'État n'est pas censé intervenir dans un marché régi par la règle de l'offre et de la demande. Dans la logique des initiateurs de cette ouverture, la libéralisation du secteur de la presse ne devait en aucun cas être synonyme d'une dérégulation intégrale. En effet, même si des gestes de forte portée politique ont été faits, tels que la dissolution du ministère de l'Information, l'État a refusé de se retirer définitivement et s'est octroyé le rôle de régulateur.

En trente années d'existence, le marché algérien de la presse écrite est animé par une centaine d'organes, entre quotidiens et hebdomadaires. Ainsi, selon les statistiques arrêtées en 2015, la presse écrite totalise 321 titres dont 149 quotidiens assurant un tirage moyen de 2,5 millions d'exemplaires (Les Cahiers de la communication, 2015, p. 6). Un foisonnement qui dénote une ouverture incontrôlée, mais aussi une double extension de ce marché de la presse: celui des lecteurs et celui des annonceurs. En effet, le lectorat de la presse s'est élargi à la faveur d'une croissance démographique et d'une montée de la scolarisation, tandis que l'espace des annonceurs se caractérise par l'apparition de nouveaux acteurs venant disputer aux annonceurs institutionnels le monopole du marché de la publicité. C'est à telle enseigne que plus de la moitié des insertions publicitaires dans les quotidiens nationaux est l'œuvre des entreprises privées. En 2015, le marché de la publicité représentait près de 200 millions de dollars (Ghali, 2016). 
En dépit de ce foisonnement de titres, la presse écrite traverse néanmoins une crise, qui a été aggravée par l'apparition de chaînes de télévisions privées et de la presse électronique. En premier lieu, une bonne partie de ces chaînes qui ont vu le jour à partir de 2011 ont été créées par des entreprises éditrices de journaux privés, qu'il s'agisse d'Ennahar TV, Echourouk TV, Al Djazairia, Dzair TV1', Numidia, Hoggar TV et Bilad TV. Dépourvues de toute assise juridique, ces chaînes émettent de l'étranger grâce à des bandes passantes. Si elles sont certes détenues par des capitaux algériens et que leurs employés travaillent sur le territoire algérien, elles ne sont pas considérées pour autant comme des entreprises de droit algérien. Ainsi, Numidia News est une entreprise de droit suisse, Atlas TV ${ }^{2}$ était et El Djazairia est de droit britannique.

En mars 2014, la loi sur l'audiovisuel a ouvert la voie à la régularisation de ces chaînes, en imposant des conditions qui ont été explicitées dans un décret d'application promulgué en août 2016. S'est ensuivie une lente régularisation: cinq chaînes ont reçu leur accréditation (El Bilad, Echourouk, Ennahar, Hoggar TV et Djazaria One). Pour autant, ces autorisations n'offrent pas la sécurité juridique nécessaire dans la mesure où les accréditations sont renouvelables chaque année et qu'à tout moment le ministère de la Communication peut les retirer.

En 2021, plus de quarante chaînes de télévision opèrent, offrant des contenus variés. Si, dans le cahier des charges de 2016, le ministère de tutelle a insisté pour que ces chaînes soient thématiques et non pas généralistes ${ }^{3}$, la plupart d'entre elles sont de fait généralistes. Certaines comme Ennahar et Echourouk se sont érigées comme les porte-voix officieux du pouvoir politique, ayant la primeur d'annoncer des décisions importantes en lieu et place des organes de l'État comme la télévision et la radio nationales ou encore l'Agence presse service (APS). Ces chaînes de télévision ont non seulement détourné des fractions entières de téléspectateurs algériens, qui étaient des récepteurs assidus de chaînes panarabes d'information,

\footnotetext{
${ }^{1}$ En raison des difficultés financières du groupe ETRHB, appartenant à I'homme d'affaires Ali Hadad, lequel est jugé dans plusieurs affaires de corruption, cette chaîne a cessé d'émettre le 25 juin 2019. De même, le journal Waqt El Jazair a cessé aussi de paraître.

${ }^{2}$ Cette chaîne a cessé d'émettre le 12 mars 2014 après une perquisition menée sur instruction du procureur de la République du tribunal d'Alger qui a ordonné la saisie du matériel de la chaîne. Cette perquisition est intervenue suite à la diffusion de l'entretien avec l'ancien dirigeant du FIS et ancien ministre Ahmed Merani jugé diffamatoire envers le président Bouteflika.

${ }^{3}$ Imposer l'option de chaînes thématiques est une manière de contrôler l'accès et la diffusion de l'information au public. Le pouvoir politique est saisi par cette obsession de contrôler l'information, car de cette façon il pourra imposer son propre récit.
} 
mais elles se sont également accaparé une part très importante du marché publicitaire: leur part représentait $84,2 \%$ des investissements publicitaires pour l'année 2015, contre 72,83\% en 2014.

Ces chaînes de télévision, dont le statut juridique reste précaire, sont désormais en concurrence avec les sites d'information en ligne. En effet, depuis leur apparition au début des années 2000, ces supports numériques se sont imposés comme l'une des sources d'information les plus sollicitées par les Algériens, notamment pendant le soulèvement populaire du 22 février 2019 avec des sites come TSA, Maghreb Émergent, Casbah Tribune, Interlignes, entre autres. Certes, la première génération des contenus informationnels en ligne était en grande majorité une simple extension du journal imprimé. Toutefois, avec le développement des technologies et la généralisation de l'internet, le contenu en ligne a commencé à pénétrer progressivement les foyers. Même si la part des sites en ligne reste très modeste en comparaison des versions électroniques des journaux papier. Pour une multitude de raisons (technologiques, économiques, notamment l'absence de rentabilité ${ }^{4}$, juridiques et politiques), les pure players n'arrivent pas à s'imposer face aux versions électroniques des journaux papier. Des progrès technologiques ont certes été réalisés, mais cela reste insuffisant pour permettre l'expansion de ces sites, à travers notamment la mise en place du paiement en ligne.

Par ailleurs, même si le cadre juridique a été étoffé avec la promulgation d'une nouvelle loi en novembre 2020, renforçant ainsi la loi organique de 2012 qui reste très généraliste dans sa formulation, il n'en reste pas moins que la contrainte politique demeure très prégnante. Elle explique à son tour le retard de la presse électronique. D'ailleurs, depuis le 22 février 2019, des dizaines de sites ont vu le jour, et la plupart d'entre eux ont soutenu le soulèvement populaire. Cet engagement en faveur du hirak et le ton très critique vis-à-vis du pouvoir politique ont valu à certains sites, comme Maghreb Émergent, Radio M, TSA et Interlignes une censure qui a rappelé les pratiques des années 1990. Last but not least, ces pure players sont considérés comme des sites étrangers, ce qui les rend d'ailleurs vulnérables face aux pressions politiques et économiques exercées par le pouvoir. Ces difficultés rencontrées par la presse électronique, ainsi que les contraintes auxquelles font face la presse écrite et la presse audiovisuelle, sont symptomatiques d'une ouverture qui a été décidée sous la contrainte.

\footnotetext{
${ }^{4}$ Certains de nos interlocuteurs nous ont avoué les obstacles que rencontrent leurs entreprises pour survivre économiquement, et notamment la difficulté d'imposer le modèle payant.
} 


\section{La régulation des médias en Algérie: dispositif juridique et institutionnel}

La dérégulation a des conséquences majeures, à la fois organisationnelles et financières. La loi organique de 2012 a institué des organes de régulation de la presse écrite et de la presse audiovisuelle. Le 14 mars 2014, le décret d'application encadrant le secteur audiovisuel a défini les missions et prérogatives de l'Autorité de régulation de l'audiovisuel (ARAV) ainsi que les conditions requises pour la création d'un service de communication audiovisuelle. Pour la presse écrite, le décret d'application définissant les missions et les prérogatives de cette instance n'a toujours pas été promulgué en 2021.

\section{Une régulation audiovisuelle très politique}

L'autorité de régulation audiovisuelle a été juridiquement instituée en janvier 2012, plus précisément dans l'article 64 de la loi organique relative à l'information promulguée à cette date: "Il est institué une autorité de régulation de l'audiovisuel, autorité indépendante jouissant de la personnalité morale et de l'autonomie financière» (JORADP, 2012, p. 23). II s'agit d'un organe ne dépendant pas du pouvoir politique et soustrait à toute interférence de quelque nature que ce soit, financière notamment. Dans le cas d'espèce, le gouvernement algérien a voulu mimer les modèles appliqués dans certains pays européens dans lesquels les instances de régulations jouent leur rôle de zone-tampon, ou plus exactement de contrepouvoir. D'ailleurs, les missions qui sont dévolues à cette instance l'illustrent. En effet, l'article 54 du décret d'application du 24 février 2014 (JORADP, 2014 , p. 11) énonce que «l'autorité de régulation de l'audiovisuel a pour missions notamment de: veiller au libre exercice de l'activité audiovisuelle dans les conditions définies dans la présente loi et par la législation et la réglementation en vigueur; veiller à l'impartialité des personnes morales exploitant les services de communication audiovisuelle relevant du secteur public; veiller à garantir l'objectivité et la transparence; veiller à la promotion et au soutien des deux langues nationales et de la culture nationale; veiller, par tous moyens appropriés, au respect de l'expression plurielle des courants de pensée et d'opinion dans les programmes des services de diffusion sonore et télévisuelle, notamment lors des émissions d'information politique et générale; veiller à ce que tous les genres de programme présentés par les éditeurs de services de communication 
audiovisuelle reflètent la diversité culturelle nationale; veiller au respect de la dignité humaine; veiller à la protection de l'enfant et de l'adolescent; veiller à ce que les événements nationaux d'importance majeure définis par voie réglementaire ne soient pas retransmis en exclusivité de manière à priver une partie importante du public de la possibilité de les suivre en direct ou en différé sur un service de télévision à accès libre».

À quelques exceptions près, les attributions dans leur ensemble ne sont en rien différentes de celles imparties à une instance de régulation dans une démocratie. Il s'agit, en fait, d'un gouvernement en miniature, qui combine à la fois le pouvoir législatif (promulgation des lois), judiciaire (règlement des différends) et exécutif (veiller au renforcement et à l'application des lois). D'ailleurs, dans la loi de 2014, l'agencement des missions de l'ARAV se décline de la manière suivante. En premier lieu, elle dispose d'un pouvoir de régulation, l'article 55 stipulant que I'ARAV «instruit les demandes de création de services de communication audiovisuelle et se prononce sur leur recevabilité; octroie les fréquences mises à sa disposition par l'organisme public chargé de la télédiffusion, en vue de la création de services de communication audiovisuelle terrestre dans le cadre des procédures définies par la présente loi; applique les règles relatives aux conditions de production, de programmation et de diffusion des émissions d'expression directe ainsi que des émissions des médias audiovisuels lors des campagnes électorales, conformément à la législation et à la réglementation en vigueur; applique les modalités de diffusion des émissions consacrées aux formations politiques et aux organisations nationales syndicales et professionnelles agréées; fixe les conditions dans lesquelles les programmes de communication audiovisuelle peuvent comporter des placements de produits ou des émissions de télé-achat; fixe les règles relatives à la diffusion des messages d'intérêt général émis par les pouvoirs publics» (JORADP, 2014, p. 12). Cette démarche est contractuelle, comme le stipule l'article 37 de la même loi (JORADP, 2014, p. 9): «Le titulaire de l'autorisation de création d'un service de communication audiovisuelle est tenu de conclure avec l'organisme public chargé de la télédiffusion un contrat ayant pour objet la transmission et la diffusion de programmes sonores ou télévisuels. » La contractualisation est également présente dans les décrets d'application $\mathrm{n}^{\circ}$ 16-220 (conditions et modalités de mise en œuvre de l'appel à candidature pour l'octroi de l'autorisation de création d'un service de communication audiovisuelle thématique) (JORADP, 2016, p. 3-5), n 16-221 (notamment le volet relatif au versement de la contrepartie financière) (JORADP, 2016, p. 6-7) et enfin $n^{\circ} 16-222$ du 11 août 2016 (règles imposables au service 
de communication sonore) (JORADP, 2016, p. 7-14). La démarche n'est pas novatrice en soi, puisque la HAICA en Tunisie les applique (Klaus, 2015).

En deuxième lieu, l'autorité de régulation dispose d'un pouvoir de contrôle. Le même article 55 de la loi de 2014 détaille cette fonction en précisant que celle-ci «veille à la conformité aux lois et règlements en vigueur de tout programme audiovisuel diffusé, quel que soit le support utilisé; contrôle, en coordination avec l'organisme public chargé d'assurer la gestion de l'utilisation du spectre des fréquences radio-électriques et avec l'organisme chargé de la télédiffusion, I'utilisation des fréquences de radiodiffusion, en vue de prendre les mesures nécessaires pour assurer une bonne réception des signaux; s'assure du respect des quotas minimums réservés à la production audiovisuelle nationale et à l'expression en langues nationales; exerce un contrôle, par tout moyen approprié, sur l'objet, le contenu et les modalités de programmation des émissions publicitaires; veille au respect des principes et règles applicables à l'activité audiovisuelle ainsi qu'à l'application des cahiers des charges» (JORADP, 2014, p. 12). À maintes reprises, I'ARAV a dû intervenir pour rappeler à l'ordre des chaînes de télévision privées en raison de la diffusion de certains contenus. Les mises en garde adressées ont contraint des chaînes privées à arrêter parfois des programmes. Ce fut le cas de l'émission du prédicateur Chemssedine el Djazairi (Chemssedine Bouroubi de son vrai nom) sur Ennahar TV et de la chaîne El Hayat TV, après la diffusion d'une interview de l'ancien député du Rassemblement pour la culture et la démocratie (RCD), Nouredine Ait-Hamouda ${ }^{5}$. Dans le cas d'espèce, l'ARAV a dû faire un travail de police, mission qu'elle a été incapable d'accomplir en 2014 lors de la campagne pour l'élection présidentielle lorsque la chaîne Ennahar s'en est prise au candidat Ali Benflis et a défendu ouvertement le candidat-président Abdelaziz Bouteflika.

En troisième lieu, l'autorité de régulation est en charge du règlement des différends. L'article 55 de la loi de 2014 (JORADP, 2014, p. 12) mentionne en effet que I'ARAV «arbitre les litiges opposant les personnes morales exploitant un service de communication audiovisuelle, soit entre elles, soit avec les usagers; instruit les plaintes émanant des partis politiques, des

\footnotetext{
${ }^{5}$ Fils de I'ancien colonel Amirouche, mort pendant la guerre de libération, Nouredine Ait-Hamouda s'en est pris dans un entretien avec le directeur de cette chaîne à des symboles de I'histoire algérienne à l'instar de l'Émir Abdelkader et l'ancien président Houari Boumedienne. Suite à la plainte déposée par la famille de l'Émir Abdelkader, Nouredine Ait-Hamouda a été mis sous mandat de dépôt et la chaîne suspendue par l'ARAV pour une période d'une semaine.
} 
organisations syndicales et/ou des associations et toute autre personne physique ou morale, faisant état de violation de la loi par une personne morale exploitant un service de communication audiovisuelle». Au cours des deux dernières années, I'ARAV a eu à instruire de nombreuses plaintes émanant de personnes physiques et morales au sujet de certains contenus diffusés, ce qui a entraîné des mises en garde et la suspension de certains programmes.

En quatrième lieu, I'ARAV dispose d'une fonction consultative comme l'indique ladite loi de 2014 (JORADP, 2014, p. 12): elle "formule des avis sur la stratégie nationale de développement de l'activité audiovisuelle; formule des avis sur tout projet de texte législatif ou réglementaire concernant l'activité audiovisuelle; formule des recommandations pour le développement de la concurrence dans le domaine des activités audiovisuelles; participe, dans le cadre de consultations nationales, à la définition de la position de l'Algérie dans les négociations internationales sur les services de diffusion sonore et télévisuelle, relatives notamment aux règles générales d'attribution des fréquences; coopère avec les autorités ou organismes nationaux ou étrangers ayant le même objet; formule des avis ou des propositions sur la fixation des redevances d'usage des fréquences radio-électriques dans les bandes attribuées au service de radiodiffusion; formule un avis, sur demande d'une juridiction, sur tout contentieux portant sur l'exercice de l'activité audiovisuelle».

Par ailleurs, le décret d'application n 16-220 du 11 août 2016 conforte I'ARAV dans son statut d'organe producteur de normes réglementaires. Pour une instance de régulation, les cahiers des charges constituent la pièce-maîtresse au sens où ils redéfinissent les fondements du contrôle du secteur, créant une relation contractuelle entre les opérateurs et l'instance de régulation (Klaus, 2015, p. 300-301). Mais est-ce réellement le cas en Algérie?

L'article 40 de la loi 04-14 du 24 février 2014 (JORADP, 2014, p. 10) stipule que «l'octroi de l'autorisation de création d'un service de communication audiovisuelle donne lieu à la conclusion, entre l'autorité de régulation de l'audiovisuel et le bénéficiaire, d'une convention qui fixe les conditions d'exploitation de l'autorisation, conformément aux dispositions de la présente loi et aux clauses du cahier des charges générales». Ces conditions sont d'ailleurs clarifiées dans les décrets d'application d'août 2016. La fonction de producteur de normes est notamment déclinée dans plusieurs textes: le chapitre 2 relatif aux conditions de mise en œuvre de l'appel à 
candidatures (articles 5, 6, 7, 8, 9 et 10) du décret d'application $n^{\circ} 16-220$ du 11 août fixant les conditions et les modalités de mise en œuvre de l'appel à candidatures pour l'octroi de l'autorisation de création d'un service de communication audiovisuel; le décret d'application $n^{\circ}$ 16-221 fixant le montant et les modalités de versement de la contrepartie financière liée à l'autorisation de création d'un service de communication audiovisuelle thématique; le décret d'application n 16-222 fixant les règles imposables à tout service de diffusion télévisuelle ou de diffusion sonore. Ce décret contient des restrictions et des interdits au sujet des contenus que ces services doivent diffuser (articles 7, 8, 9) et des dispositions ayant trait à l'éthique et à la déontologie (de l'article 10 à l'article 26). Le décret est enfin plus explicite en ce qui a trait aux fonctions de contrôle et de règlement de litiges que cette instance doit assumer.

\section{La presse écrite en attente d'une instance de régulation pourtant prévue par les textes}

La décision de créer une autorité de régulation de la presse écrite constitue une première dans l'histoire de la presse algérienne depuis I'Indépendance. Cette décision semble être dictée par le souci de réguler un marché connu pour son abondance de titres aux contenus informationnels hétéroclites. Plus important encore, il s'agit de restructurer un marché dérégulé par une libéralisation que même la batterie de textes adoptés depuis le début des années 1990 n'a pas su juguler.

Plus concrètement, comme le stipule l'article 40 de la nouvelle loi organique sur l'information (JORADP, 2012, p. 21), l'autorité de régulation de la presse écrite est chargée notamment: «d'encourager la pluralité de l'information, de veiller à la diffusion et à la distribution de l'information écrite à travers tout le territoire national, de veiller à la qualité des messages médiatiques ainsi qu'à la promotion et la mise en exergue de la culture nationale dans tous ses aspects, de veiller à l'encouragement et à la consolidation de la publication et de la diffusion dans les deux langues nationales par tous les moyens appropriés, de veiller à la transparence des règles économiques de fonctionnement des entreprises éditrices, de veiller à l'interdiction de la concentration des titres et organes sous I'influence financière, politique ou idéologique d'un même propriétaire, de fixer les règles et les conditions des aides accordées par l'État aux organes d'information et de veiller à leur répartition, de veiller au respect des normes en matière de publicité et d'en contrôler l'objet et le contenu, de recevoir 
des déclarations comptables des publications périodiques autres que celles générées par l'exploitation, de recueillir, auprès des administrations et des entreprises de presse, toutes les informations nécessaires pour s'assurer du respect de leurs obligations».

Sa composition renvoie à un mode paritaire, comme le précise l'article 50 (JORADP, 2012, p. 22) : "L'autorité de régulation de la presse écrite est composée de quatorze (14) membres nommés par décret présidentiel et ainsi désignés: trois (3) membres désignés par le président de la République dont le président de l'autorité de régulation, deux (2) membres non parlementaires proposés par le président de l'Assemblée populaire nationale, deux (2) membres non parlementaires proposés par le président du Conseil de la Nation, sept (7) membres élus à la majorité absolue parmi les journalistes professionnels justifiant d'au moins quinze (15) ans d'expérience dans la profession.»

Comme on peut le voir, les missions assignées à cette instance ainsi que sa composition vont dans le sens d'une réorganisation d'un marché que l'État qualifie de dérégulé et subissant des dysfonctionnements. De surcroît, l'annonce de sa création intervient à un moment où le marché mondial de la presse connaît d'importantes mutations, dont les plus saillantes restent la tendance vers les fusions-concentrations et la numérisation des journaux. II s'agit en somme d'une évolution d'autant plus logique que le marché de la presse écrite en Algérie connaît une désorganisation et une crise entraînant la fermeture de plusieurs titres.

\section{La presse électronique et les obstacles à son développement}

Le retard dans la mise en place d'une plateforme technologique permettant la numérisation des supports et les pesanteurs politiques sont parmi les facteurs expliquant les lenteurs dans l'émergence de la presse numérique en Algérie (Dris, 2017, p. 271-278). Il a fallu que la presse écrite se lance dans la digitalisation et que certains sites électroniques soient créés, comme par exemple Algérie Interface en 2001, pour que la reconnaissance de la presse en ligne comme une réalité juridique s'impose. Ainsi, ladite loi organique de 2012 énonce dans son article 66 (JORADP, 2012, p. 23) que «l'exercice de l'activité d'information en ligne est libre». Plus explicite, l'article 67 (JORADP, 2012, p. 23) définit cette presse électronique comme: «tout service de communication écrite en ligne destiné au public ou une catégorie de public, édité à titre professionnel par une personne physique ou morale de droit algérien qui a la maîtrise de la ligne éditoriale de son 
contenu ». L'article 68 (JORADP, 2012, p. 23) délimite son contenu en précisant que: "l'activité de presse écrite en ligne consiste en la production d'un contenu original, d'intérêt général, renouvelé régulièrement, composé d'informations ayant un lien avec l'actualité et ayant fait l'objet d'un traitement à caractère journalistique» (article 68). Sont donc exclus de cette catégorie «les publications diffusées en version papier (...) lorsque la version mise en ligne et la version originale sont identiques.»

Depuis la promulgation de cette loi, des dizaines de sites en ligne ont vu le jour. En 2021, ils sont plus de 150 à être en activité, non sans rencontrer des difficultés multiples: manque de reconnaissance institutionnelle; difficultés d'accès aux sources d'information, difficultés dues au marché publicitaire et, enfin, censure. Ce dernier point constitue une épée de Damoclès. Depuis le début de l'année 2020, plusieurs sites importants ont été censurés: Maghreb Émergent, TSA, Interlignes, Casbah Tribune, pour ne citer que ceux-là. En filigrane, cette censure pose la lancinante question de la sécurité juridique qui devrait être accordée aux sites d'information en ligne et à la régulation du secteur.

En promulguant le décret d'application sur la presse électronique le 25 novembre 2020 (JORADP, 2020, p. 12-17), les pouvoir publics en Algérie espèrent clore la parenthèse d'un «mariage blanc» avec la presse électronique. En substance, le nouveau décret énonce certaines règles procédurales conditionnant l'octroi d'autorisation pour la création d'un site d'information en ligne. II s'agit de conditions à la fois techniques, statutaires, administratives et judiciaires. Plus précisément, les candidats au lancement d'un site d'information électronique doivent, entre autres, être de nationalité algérienne, détenir un diplôme universitaire et avoir pour expérience de trois ans dans le domaine de la presse (article 5). Le capital de l'entreprise doit être algérien (article 4), et une «même personne physique ou morale de droit algérien ne peut posséder, contrôler ou diriger plus d'un organe d'information générale en ligne. Une même personne physique ou morale de droit algérien ne peut être actionnaire dans plus d'un organe d'information générale en ligne» (article 8). II est également exigé une extension du nom du domaine «.dz» «pour tout site dont l'hébergement est exclusivement domicilié physiquement et logiquement en Algérie» (article 6). Si ces conditions peuvent paraître contraignantes, elles le sont moins comparativement à celle qui exige (article 5, alinéa 5) du directeur responsable de l'organe d'information en ligne de «n'avoir pas fait l'objet de condamnation pour crimes de diffamation, d'injure, d'insulte, d'outrage, de discrimination ou de haine et d'incitation à ces crimes». 
Le nouveau texte précise également la création d'une autorité de régulation de la presse électronique. Celle-ci aura pour tâche de réguler cette activité, notamment l'octroi d'un certificat d'enregistrement (article 25) et l'imposition de procédures administratives (mise en demeure, suspension provisoire et retrait du certificat d'enregistrement) pour non-respect des dispositions visées dans le présent décret (article 32). Il reste à savoir si cette autorité de régulation de la presse électronique subira le même sort que celle de la presse écrite, celle-ci n'existant jusqu'alors que dans les textes.

La promulgation de ce décret spécifique participe, en apparence, de la volonté des pouvoirs publics algériens de réguler un secteur très instable. Les difficultés rencontrées par ces sites d'information pour accéder aux sources d'information, mais aussi au marché des annonceurs lui-même dérégulé faute d'un dispositif légal adapté, expliquent sans doute ce souci de promulguer un texte et d'annoncer la création d'une autorité de régulation. En Tunisie et au Maroc, la même démarche a été entreprise, ce qui offre une certaine sécurité juridique aux médias en ligne.

Soulever cette question en Algérie, c'est soulever aussi les enjeux sousjacents à ce processus de régulation des médias. En filigrane se pose la question des contraintes qui se dressent devant la mise en place de ces mécanismes de régulation consacrés dans les textes législatifs. Ce cadre juridique proposé est-il adapté à la réalité du terrain? Vise-t-il réellement à introduire de l'ordre dans un marché médiatique en manque de modèle économique? La configuration politique, montrant un refus du régime d'opérer une vraie ouverture, n'entrave-t-elle pas la mise en place de réels mécanismes de régulation?

\section{Une régulation sous contraintes}

\section{Un cadrage juridique en décalage avec les réalités du terrain}

S'il est admis que la production de normes juridiques est l'un des marqueurs essentiels de toute démarche de régulation, il n'en demeure pas moins vrai que celles-ci se doivent d'être adaptée à la réalité du terrain. Or, dans le cas algérien, les textes promulgués semblent être largement en décalage vis-à-vis d'un paysage en mutation constante. Ainsi les décrets de 2014 et de 2016 ont-ils été promulgués justement pour encadrer le champ de l'audiovisuel composé essentiellement de chaînes privées dont l'existence légale n'est pas reconnue. Selon Zouaoui Benhamadi, ancien président de 
I'ARAV, «le paysage audiovisuel actuel, en dehors du public, est composé totalement d'institutions médiatiques qui n'obéissent ni à la loi générale ni à la pratique commerciale ni aux contraintes d'une institution économique ${ }^{6}{ }^{\prime}$. De fait, "peut-on sanctionner une chaîne de télévision de droit étranger?", s'interroge-t-il ${ }^{7}$. En effet, les chaînes qui ont été autorisées à opérer en 2012 n'avaient pas d'existence légale. Elles sont de droit suisse ou britannique, ce qui ne va pas sans soulever des questions au sujet de leur financement, du personnel qu'elles emploient et des contenus qu'elles proposent. En 2016, quand les décrets d'application ont été édictés, seules 5 chaînes ont reçu leurs accréditations, mais en tant que bureaux de chaînes étrangères. La nuance est de taille puisque, dans les faits, ces chaînes fonctionnent plus comme des chaînes généralistes, pour la plupart d'entre elles, que comme des bureaux de chaînes étrangères. Autrement dit, des questions relatives à leur mode de fonctionnement (économique) et leur proximité avec le pouvoir politique suscitent des interrogations légitimes.

À l'évidence, le cadrage juridique ne reflète pas cette volonté de laisser I'ARAV jouer pleinement son rôle de régulateur. Car aussi importantes que soient ses prérogatives et missions, I'ARAV renvoie l'image d'un appendice du pouvoir exécutif qui l'actionne à sa guise. Deux indices l'attestent. Tout d'abord, le chevauchement des prérogatives de I'ARAV avec celles du ministère de la Communication. En effet, dans le décret d'application $n^{\circ}$ 11-216 du 12 juin 2011 fixant ses missions et prérogatives, ce dernier doit "veiller à la régulation des activités de communication, y compris celles liées aux médias électroniques (journaux, radios et télévisions sur internet) de concert avec les entités de régulation (...) délivrer les autorisations d'exercice des activités de communication y compris la presse électronique (journaux, télévisions, radios)» (article 2) (JORADP, 2011, p. 7). Et pour mieux conforter la position du ministre, l'article 22 du décret d'application $n^{\circ}$ 16-220 de 2016 (JORADP, 2016, p. 5) indique que le «ministre chargé de la Communication peut, à tout moment, après consultation de l'autorité de régulation de l'audiovisuel, décider de mettre un terme au processus d'octroi d'autorisation(s). Cette décision, motivée, est communiquée par l'autorité de régulation de l'audiovisuel à l'ensemble des soumissionnaires ». Ensuite, la composition de l'ARAV elle-même traduit cette forte dépendance politique. En effet, c'est le mode de nomination politique qui prévaut: 5 membres sont désignés par le président de la République dont le président

\footnotetext{
${ }^{6}$ Entretien, Alger, 10 janvier 2021.

${ }^{7}$ Ibid.
} 
de I'ARAV, 2 par le Parlement et 2 par le Sénat. La logique paritaire est donc exclue, contrairement à la HAICA en Tunisie, dans laquelle les syndicats des journalistes et l'association des patrons des médias sont représentés. Ce mode de désignation est un marqueur fort de la logique tutélaire que l'exécutif en Algérie veut exercer sur l'ARAV. En fait, comme le souligne Rachid Zouaimia, l'indépendance de cette institution revêt un caractère virtuel (Zouaimia, 2018, p. 761). Le pouvoir politique se montre très rétif à l'idée de voir le paysage audiovisuel se soustraire à son contrôle, tant l'influence réelle ou supposée qu'il peut ou pourrait exercer dans le façonnement d'une «opinion publique» qui lui est hostile n'est pas à négliger.

Par ailleurs, les missions de contrôle et d'arbitrage devraient être, elles aussi, nuancées. En effet, I'ARAV n'a pas été en mesure d'imposer son autorité lorsque les chaînes publiques ont diffusé des informations erronées et diffamatoires sur certains acteurs politiques et des figures historiques. C'est le cas du commandant Lakhdar Bouragaa ${ }^{8}$, qui a été accusé en 2019 par la télévision publique d'avoir usurpé l'identité de son frère lors de la Guerre de libération. De même, pendant les élections législatives de 2017, cette instance n'a pas non plus fait respecter le droit des chaînes privées d'organiser des débats de campagne, le ministre de la Communication de l'époque, Hamid Grine, ayant signé une circulaire leur interdisant de le faire. Les seules fois où l'instance de régulation est intervenue, c'est lorsque des chaînes privées ont diffusé en 2020 des programmes jugés contraires à la morale ou que des présentateurs vedettes ont pris des positions sur des questions considérées comme tranchées ${ }^{9}$ par les pouvoirs publics.

Contrairement au secteur audiovisuel, les secteurs de la presse papier et de la presse en ligne n'ont pour l'instant pas été dotés d'instances de régulation. Pourtant, les textes juridiques (la loi organique de 2012 et le dernier décret d'application sur la presse électronique en 2020) cités précédemment les mentionnent explicitement. Pour les pouvoirs publics algériens, le retard pris dans la mise en place de ces instances s'expliquerait essentiellement par les dysfonctionnements du secteur, notamment en matière de publicité (APS, 23 novembre 2020). Ainsi, pour l'autorité de

\footnotetext{
${ }^{8}$ Lakhdar Bouragaa, ancien commandant de l'Armée de libération nationale, a été mis en détention pour avoir osé critiquer l'état-major de l'armée en 2019. Libéré de prison, il est décédé le 5 novembre 2020.

${ }^{9}$ Le cas du prédicateur Chemssedine de la chaîne Ennahar qui avait contesté une fatwa (un avis religieux) émise par le ministère des Affaires religieuses qui autorise à donner la zakat (aumône) de l'Aïd durant le ramadan et non le jour fixé. Le téléprédicateur a été suspendu au mois de mai 2020 par la chaîne Ennahar. Depuis il officie sur la chaîne Echourouk TV.
} 
régulation de la presse écrite, qui aurait un caractère paritaire (14 membres: 7 désignés et 7 élus par leurs pairs), le problème de l'identification des journalistes se pose avec insistance. Qui est journaliste et qui ne l'est pas? Afin de mettre un terme à cette anarchie et préparer le terrain pur I'installation de cette instance, une commission chargée de la délivrance de la carte de presse a été créée. Instituée à l'époque où Hamid Grine, ancien journaliste, était ministre de la Communication, celle-ci a été chargée, d'une part, d'identifier les journalistes et leur délivrer des cartes, d'autre part, de préparer les élections pour élire les membres de l'autorité de la presse écrite ${ }^{10}$. En réalité, le retard pris dans la mise en place de cette instance s'explique davantage par les enjeux qu'elle suscite. Son caractère paritaire lui confère a priori une certaine autonomie dans la régulation d'un secteur dominé par quelques gros titres, qui plus est très critiques envers le pouvoir politique. De surcroît, comme il est mentionné dans ses missions, cette autorité doit intervenir pour aider à la structuration d'un marché de la publicité dont le cadrage légal date de 1986. C'est dire l'ampleur des obstacles qui se dressent devant elle, au moment même où le secteur de la presse papier traverse une des crises les plus sévères de son histoire. L'installation de cette instance soulèverait de facto la question de la raison d'être d'un ministère chargé de la Communication ${ }^{11}$.

La tâche s'annonce encore plus ardue dans le cas de la presse électronique. Les autorités algériennes ont fait en sorte de produire un texte contenant des critères d'éligibilité, mais aussi l'engagement de doter la corporation d'une instance de régulation dans laquelle les journalistes seraient représentés. Dans l'absolu, un cadre juridique offre une sécurité aux acteurs opérant dans ce secteur. Cependant, les expériences du code de l'information de 1990 et de la loi organique de 2012 laissent planer des doutes sur les garanties que le texte sur la presse électronique pourrait offrir. En effet, non seulement les modèles économiques des sites d'information ne sont pas assez visibles, mais cette nouvelle loi renvoie également l'image d'un pouvoir qui veut contrôler ce secteur. D'ailleurs, certains acteurs soutiennent que ce cadre juridique n'offre pas de sécurité suffisante aux

\footnotetext{
${ }^{10}$ Entretien par téléphone avec Idir Dahmani, ancien directeur de publication des quotidiens El Mihwer (Le hub quotidien) et El Mouatan (Le citoyen), Alger, 21 décembre 2020.

${ }^{11}$ Selon certains patrons de journaux, actuels ou anciens, dont Hassan Ouali du quotidien Liberté, ou Hassan Moali ancien directeur de publication des deux quotidiens El Waqt et Le Temps, propriété des hommes d'affaires Ali Haddad et Idir Dahmani, que nous avons interrogés, la raison d'être du ministère de la Communication se pose avec acuité.
} 
investisseurs - et ils en veulent pour preuve la presse papier ${ }^{12}$. Par ailleurs, le fait que la loi exige l'absence d'antécédents judiciaires comme critère d'éligibilité incite certains responsables de ces sites à penser que ce texte a été préparé pour de potentiels délinquants ${ }^{13}$. Cela d'autant plus que, parmi les conditions énoncées figure celle (article 5, alinéa 5) qui impose à toute personne voulant investir dans ce secteur de "n'avoir pas fait l'objet de condamnation pour crimes de diffamation, d'injure, d'insulte, d'outrage, de discrimination ou de haine et d'incitation à ces crimes». Cette condition peut paraître rédhibitoire dans la mesure où beaucoup de journalistes ont été condamnés pour diffamation ${ }^{14}$. Toutes aussi rédhibitoires sont les conditions qui imposent, d'une part, la qualité de journaliste pour ouvrir un site et, d'autre part, I'hébergement du site dans le domaine «.dz». La première entend procéder en apparence à un écrémage dans un secteur où les pouvoirs publics et les journalistes en poste se plaignent du développement des producteurs non professionnels. Or, dans un marché médiatique libéral et concurrentiel, le domaine de la presse, quel que soit le support, est censé être ouvert à tout investisseur même s'il n'appartient pas à la corporation des journalistes. Quant à la seconde condition, les éditeurs de la presse en ligne pointent le retard technologique qui rend incongrue l'imposition du domaine «.dz» (Hammadi, 2020).

Enfin, l'instance de régulation prévue dans ce nouveau texte soulève les mêmes questionnements que pour son homologue en charge de la presse écrite, qu'il s'agisse de sa composition, de ses prérogatives et de ses missions. C'est d'autant plus flagrant que le texte fixant les missions du ministre de la Communication (2011) n'a pas été amendé.

\section{Au prisme d'un marché dérégulé}

Dans le sillage de la libéralisation de l'économie voulue par l'ancien président Abdelaziz Bouteflika et son équipe de conseillers, le secteur des médias a connu des ouvertures qui ont bouleversé sa physionomie, mais aussi les rapports qu'il entretient avec les acteurs économiques et le pouvoir politique. En effet, cette stratégie de libéralisation a permis l'émergence d'une nouvelle catégorie d'hommes d'affaires qui ont prospéré à l'aune de

\footnotetext{
12 Entretien avec Ihsan El Kadi, directeur du site Maghreb Émergent et de Radio M, Alger, 27 octobre 2020.

${ }^{13}$ Entretien par téléphone avec une responsable d'un site en ligne, Alger, 19 décembre 2020.

${ }^{14}$ Entretien par téléphone avec Hadjer Guennafa, directrice du site en ligne L'Info, Alger, 14 décembre 2020.
} 
la commande publique favorisée par la rente pétrolière. Cette ascension économique devait avoir un prolongement médiatique avec l'acquisition de journaux et la création de chaînes de télévision. De fait, le marché des médias en Algérie, en dehors du public, est animé par des acteurs aux profils hétéroclites: journalistes-entrepreneurs et hommes d'affaires qui veulent avoir des relais médiatiques. Cette hétérogénéité produit à son tour des positionnements économiques et politiques qui ressemblent, à quelques exceptions près, au modèle marocain (Benchenna, Marchetti, 2019).

Pour le profil des journalistes-entrepreneurs, le cas le plus classique est représenté par la première génération des journalistes qui ont fait leur carrière dans les médias du parti unique et puis se sont lancés dans "l'aventure intellectuelle» initiée par l'ancien chef du gouvernement, Mouloud Hamrouche. Grâce aux aides fournies par le gouvernement, d'anciens journalistes (Dris, 2017), à l'instar d'Omar Belhouchet, Khieredine Ameyar, Chérif Rezki, Zoubir Souissi, Ahmed Fatani, Mohamed Benchicou, Abdelkrim Djaad notamment, ont lancé leurs propres journaux: Liberté, El Watan, Le Soir d'Algérie, El Khabar, Le Matin, etc. Cette expérience a produit à son tour une deuxième catégorie de journalistes-entrepreneurs, puisque des journalistes salariés de certains de ces journaux ont créé à leur tour leur propre titre: Abdelouahab Djakoun (La Nouvelle République), Ihsane El Kadi (Maghreb Émergent), Lounas Guemach (TSA), Anis Rahmani (Ennahar et Ennahar TV), Ali Foudhil (Echourouk, Echourouk TV). Enfin, la nouvelle génération est constituée de jeunes journalistes qui ont préféré opter pour la presse en ligne: Khaled Drareni (Casbah Tribune), Ghada Hamrouche (Algérie 24), Hadjer Guenanfa (L'Info), Mohamed Rabah (Sabq press), Mohamed Lamine Maghnine (Tariq News) et Abdou Semar (Algériepart), pour ne citer que ceux-là.

Les hommes d'affaires, qui sont désormais nombreux, opèrent dans des secteurs aussi divers que les travaux publics, le transport et le montage des véhicules, l'agroalimentaire, les services, etc. On peut citer par exemple les cas emblématiques d'Issadf Rebrab (le quotidien Liberté), d'Ali Hadad (Dzair TV et Dzair News, Le Temps et El Waqt avant leur fermeture), des frères Aissiou et d'Ould Zemirli, travaillant dans I'immobilier et l'agroalimentaire (Djazaia One), de Mahiedine Tahkout (Numedia News TV). Les hommes politiques sont également un autre type de profil très représenté: Amara Benyounès, secrétaire général du Mouvement populaire algérien (MPA) (La Dépêche de Kabylie), Miloud Chorfi, ancien sénateur du RND ${ }^{15}$ (El Adjwa),

\footnotetext{
${ }^{15}$ Décédé le 20 février 2017, Miloud Chorfi était aussi le premier président de l'ARAV.
} 
Abdelhamid Si Affif, député du FLN (Manbar el koraa et La Tribune des lecteurs), le général à la retraite Khaled Nezar (le site en ligne Algérie patriotique) (Ahcène-Djaballah, 2019).

Cette diversité des acteurs et de leurs profils renvoie l'image d'un marché concurrentiel et diversifié. Toutefois, cette apparente pluralité cache un espace médiatique déstructuré et dérégulé, animé par des entreprises dont les modèles économiques demeurent flous. Et les illustrations ne manquent pas dans le cas de l'audiovisuel. En effet, hormis les chaînes publiques, les chaînes privées, opérant depuis quelques années, ne sont en réalité que des chaînes off-shore, de simples bureaux de chaînes étrangères qui sont censés n'employer que dix personnes tout au plus, dont des journalistes. Or, les cinq chaînes accréditées emploient en moyenne entre 150 et 200 personnes, ce qui constitue en soi une infraction caractérisée à la législation en vigueur ${ }^{16}$. Dans certains cas, la gestion financière n'obéit pas aux règles de management d'une entreprise économique. Ainsi, pour ne citer que le cas du groupe Le Temps Médias, qui regroupait les deux chaînes Dzair TV et Dzair News ainsi que les deux journaux du même nom, c'est le groupe ETRHB détenu par Ali Hadad qui payait les salaires des journalistes, les créances auprès des entreprises publiques et aussi les producteurs auxquels ils ont acheté des programmes ${ }^{17}$.

Quant aux journaux de la presse écrite et ceux de la presse électronique, les difficultés financières sont encore plus prononcées: baisse des recettes publicitaires, baisse du tirage et problèmes de distribution. Pour la presse électronique, la situation est d'autant plus critique dans la mesure où elle n'a pas accès au marché de la publicité, hormis quelques pure players, qui parviennent à survivre grâce aux régies publicitaires globales ${ }^{18}$ (comme Google ou Amazon).

Ces difficultés rencontrées par les acteurs de la presse papier et électronique soulèvent de nouveau la question de la distribution de la publicité, publique notamment. Si la publicité commerciale est censée obéir aux règles du marché, elle demeure néanmoins restreinte en raison de l'impact de la crise économique qui frappe l'Algérie depuis 2014, du fait de la chute des prix du pétrole. Ce qui n'a pas manqué de créer un climat de concurrence avec les chaînes de télévision privées, qui absorbent une part

\footnotetext{
${ }^{16}$ Entretien avec Zouaoui Benhamadi, Alger, le 11 janvier 2021.

${ }^{17}$ Entretien avec Abrous Outoudert, Alger le 23 décembre 2020.

${ }^{18}$ Entretien avec Ihsan El Kadi, Alger le 27 octobre 2019.
} 
assez conséquente de ce marché $(84,2 \%$, soit 73 millions d'euros, rien que pour l'année 2015). De plus, cette crise a non seulement renforcé le poids de la publicité publique, mais elle a aussi consacré l'Agence nationale d'édition et de publicité (ANEP) comme un courtier servant à rétribuer la clientèle du régime politique et à sanctionner les médias dissidents. Quelques exemples suffisent à montrer cette logique clientéliste: les journaux El Balagh et El Balagh Erriadi ${ }^{19}$ (qui sont la propriété de l'ancienne star du football algérien, Rabah Madjer, très proche du clan présidentiel et très actif lors des campagnes pour les troisième et quatrième mandats); Edough, journal local paraissant dans la wilaya d'Annaba, appartenant à l'un des fils de feu le général Ahmed Gaid Salah et dont les montants reçus par l'ANEP n'ont pas été révélés. La rétribution pour allégeance politique nous est fournie par les trois exemples suivants: La Tribune des lecteurs de Abdelhamid Si Affif, ancien député du FLN ${ }^{20}$, d'El Adjwa de Miloud Chourfi, feu député du RND et ancien président de l'Arav ${ }^{21}$, et de La Dépêche de Kabylie ${ }^{22}$, fondée en juin 2002 par Amara Benyounès, ancien ministre, libéré le 2 novembre 2020 après avoir purgé une peine d'un an de prison. Enfin, les alignements éditoriaux sur la politique du pouvoir politique sont légions chez des journaux comme Ennahar, La Nouvelle République, Echourouk, El Bilad et El Hayet.

Cet usage clientéliste de la publicité publique a été pointé du doigt dans un entretien accordé au quotidien EI Watan par Larbi Ounoughi, I'ancien PDG de I'ANEP qui a été démis de ses fonctions le 27 septembre 2020: «L'ANEP a fait l'objet de monopole par des groupes d'influence au pouvoir qui ont fait main basse sur l'ANEP (...). Les services de l'ANEP étaient dans une telle anarchie indescriptible qu'il est difficile de savoir ce qui s'y est passé.» Le fait d'accuser des groupes d'influence au pouvoir est un marqueur suffisant du degré de politisation de la publicité publique, qui reste avant tout un produit commercial. «C'est la gestion politique qui l'a mise dans cet état, elle ressemble beaucoup plus à une association caritative qu'à une entreprise économique et commerciale soumise aux règles et aux normes du marché (...). L'ANEP fonctionnait au téléphone», explique Larbi Ounoughi dans cette même interview (Rabia, 2020). Au-delà des dysfonctionnements qui caractérisent le marché de la publicité, qui sont aggravés par l'absence d'un

\footnotetext{
${ }^{19} \mathrm{Ce}$ journal a reçu, à partir de 2015, 30 milliards de centimes, soit 1,9 million d'euros. Sources: Larbi Ounoughi, ancien PDG de l'ANEP, El Watan, 6 août 2020.

2028 milliards de centimes, soit 1,8 million d'euros (entre 2016 et 2019) et 31 milliards, soit 2 millions d'euros (2012-2015).

${ }^{21} 6$ milliards de centimes, soit 380000 euros.

2223 milliards de centimes, soit 1460000 euros, ibid.
} 
cadre juridique en phase avec les transformations profondes de l'univers médiatique, c'est toute la problématique des rapports entre médias et pouvoir politique qui est posée.

\section{Le poids prégnant du contrôle politique}

En instituant des autorités de régulation, les pouvoirs publics veulent donc renvoyer l'image d'une Algérie qui opérerait sa mue en créant un environnement propice à l'épanouissement des libertés individuelles et publiques. Toutefois, la régulation reste une notion abstraite, comme en témoigne l'existence de I'ARAV. Nonobstant son institutionnalisation à travers la promulgation des textes de loi lui garantissant sa sécurité juridique, cette instance reste sous l'emprise du pouvoir politique.

Tout d'abord, les textes codifiant son action revêtent un caractère vague et peuvent être facilement instrumentalisés politiquement. II est certes établi que les normes internationales relatives à la régulation du contenu médiatique imposent des restrictions sur des sujets tels que la sécurité nationale, l'ordre public, la pornographie, la protection de l'enfance, de la morale publique ou encore la diffamation (Bouziane, 2018, p. 4411). Ces restrictions sont contenues à la fois dans la loi du 24 février 2014 relative à l'audiovisuel et dans les décrets d'application d'août 2016. Toutefois, les restrictions et interdits énoncés restent flous dans leur formulation et peuvent être sujet à diverses interprétations. L'article 7 du décret d'application $\mathrm{n}^{\circ} 16-222^{23}$, qui parle de l'offense faite au président de la République, en fournit une illustration emblématique: «Dans la diffusion des programmes, les responsables des services de communication audiovisuelle veillent à proscrire toute offense, toute parole outrageante, injurieuse ou diffamatoire à l'encontre de la personne du président de la République et/ou de l'institution représentée par le président de la République». Les notions d'offense, de parole outrageante ou injurieuse et diffamatoire restent floues. Elles le sont d'autant plus que ce texte a été promulgué en 2016, c'est-àdire à une période où Abdelaziz Bouteflika était encore président de la République et que les polémiques sur son état de santé ne cessaient de se développer. On peut faire l'hypothèse que cette disposition a été imposée pour faire taire toute critique au sujet de l'état de santé du Président, de son incapacité à gérer et surtout des appels à l'application de l'article 102

\footnotetext{
${ }^{23}$ Décret d'application $n^{\circ} 16-222$ du 11 août 2016 portant cahier des charges générales fixant les règles imposables à tout service de diffusion télévisuelle ou de diffusion sonore.
} 
de la Constitution de 2016 qui précise la vacance du pouvoir pour toute une série de raisons, notamment médicales.

L'élasticité qui entoure ces notions confère au pouvoir exécutif la latitude de faire taire de toute voix discordante. D'ailleurs, cette crainte est telle que le pouvoir politique n'hésite pas à transgresser l'un des critères-phares de l'indépendance de cette instance, à savoir son autonomie vis-à-vis du pouvoir politique, en nommant en 2015 un député d'un parti au pouvoir, le RND, feu Miloud Chorfi, à la tête de I'ARAV. Pendant la campagne des élections législatives de mai 2017, le ministre de la Communication de l'époque, Hamid Grine, avait également rédigé une note interdisant aux chaînes privées d'organiser des débats sur les élections. Une décision qui n'a pas manqué de susciter l'ire du président de l'ARAV au moment des faits, Zouaoui Ben Hamadi, considérant la démarche du ministre comme une interférence flagrante (Lachichi, 2017). Enfin, le 25 mars 2019, la présidence de la République a nommé un membre de l'ARAV, Lotfi Chriet ${ }^{24}$, à la tête de la télévision nationale, également en violation flagrante des règles régissant le fonctionnement de cette instance.

Par ailleurs, en pleine crise causée par la pandémie de coronavirus, le pouvoir exécutif a menacé de publier un texte de loi criminalisant toute remise en cause de la capacité de l'État. Cette notion, très vague, peut englober les critiques à l'adresse du président de la République par exemple sur sa gestion de la crise ou toute autre action. D'ailleurs, c'est suite à un article d'opinion d'Ihsane El Kadi intitulé «Les 100 jours de malheur de Abdelmadjid Tebboune» (El Kadi, 2020) que le site Maghreb Émergent et Radio $M$ ont été censurés, et cela même si cette loi n'a pas été promulguée.

En deuxième lieu, les interdits contenus dans l'article 8 du décret d'application de $2016^{25}$, ceux touchant notamment au «respect des valeurs nationales, à l'unité nationale, aux exigences de la défense et de la sécurité nationale, au respect des autres références religieuses et des autres croyances et religions», limitent la marge de liberté des journalistes travaillant dans le secteur audiovisuel dans la mesure où ces notions revêtent un caractère élastique, sujettes donc à différentes interprétations.

\footnotetext{
${ }^{24}$ Le 25 mai de la même année, Lotfi Chriet est démis de ses fonctions par le président par intérim de l'époque, Abdelkader Bensalah. Il est réputé proche de Said Bouteflika, frère et conseiller du président, mais aussi responsable de la chaîne de télévision El Istimrariya (continuité) créée spécialement pour faire la promotion du 5e mandat.

${ }^{25}$ Décret d'application $n^{\circ} 16-222$ du 11 août 2016 portant cahier des charges générales fixant les règles imposables à tout service de diffusion télévisuelle ou de diffusion sonore.
} 
Plus restrictif, davantage, dans sa formulation, I'article $9^{26}$ énonce que «toute diffusion d'informations audiovisuelles relatives à des thèmes portant sur l'unité nationale, la sécurité et la défense nationales est soumise à l'autorisation préalable des autorités concernées». Quelles sont donc les «lignes rouges» que le journaliste ne devrait pas franchir? À défaut d'avoir des réponses claires à cette question, la plupart des journalistes se résignent le plus souvent à pratiquer l'autocensure. Le problème se pose aussi pour la presse écrite avec la loi organique de 2012, mais aussi pour la presse électronique. L'approche sécuritaire dans le traitement de l'information autorise à donner une définition extensible aux notions d'unité, de sécurité et de défense nationales, qui expliquent les poursuites récurrentes de certains journalistes et titres.

En troisième lieu, même en disposant d'un socle juridique, la presse électronique n'en est pas moins vulnérable face aux pressions du pouvoir politique. Enfin, le penchant tutélaire du pouvoir politique s'exprime également à travers cette tentative de répliquer dans l'univers journalistique l'espace politique autorisé, à savoir les partis qui font allégeance (AlMoualate) et les partis d'opposition.

Les mutations de l'espace médiatique algérien sont révélatrices des recompositions du champ politique depuis le début du processus d'ouverture politique vers la fin des années 1980. Sa régulation vise à conforter la permanence d'un régime politique qui tente par tous les moyens de renforcer sa résilience. Les instances de régulation sont un des leviers de ce contrôle politique et économique. L'État conserve une tutelle sur cet univers médiatique, tout particulièrement sur son sous-espace audiovisuel, entretenant l'illusion d'un pluralisme médiatique sans réelle diversité. En dépit des mutations politiques à l'œuvre depuis le 22 février 2019, la volonté de contrôle du pouvoir politique est tout aussi prégnante. Force est de constater que le soulèvement populaire, ou Hirak, n'a pour l'instant pas impacté profondément l'espace médiatique, les journalistes étant d'autant plus sur la défensive que l'économie du secteur est très fragilisée.

La presse électronique est confrontée à des problèmes de reconnaissance et fonctionne dans un cadre légal ne lui apportant pas une forte sécurité juridique. La régulation de la presse papier s'avère encore plus compliquée, comme le montre le retard dans l'installation de l'instance chargée d'assumer cette mission, qui est pourtant prévue par un texte. Si celui-ci renvoie à

26 lbid. 
des problèmes d'ordre organisationnel de la profession de journaliste en Algérie, il s'explique aussi surtout par des raisons politiques. Le monde de la presse papier algérienne se caractérise par une désorganisation dont le marqueur le plus visible est l'absence d'une loi sur la publicité qui cadrerait avec la réalité du marché. L'absence de cette loi est notamment à l'origine du désordre caractérisant la ventilation des annonces publicitaires entre les différents organes. Par ailleurs, la presse papier privée, du moins une partie des titres, jouit d'une certaine liberté de ton qui irrite le pouvoir politique. Le fait que cette instance prévue par la loi soit paritaire explique aussi ce retard. Tout se passe comme si la crainte de voir des journalistes connus pour leur ton critique figurer dans cette structure de régulation était une perspective que les tenants d'une approche sécuritaire redoutent.

En définitive, l'espace médiatique reste rattaché à la sphère politique. Les recompositions politiques à l'œuvre depuis ces dernières années, et notamment depuis le 22 février 2019, n'augurent pas, pour l'instant, de l'émergence d'un univers médiatique indépendant de l'ordre politique et pouvant même l'influencer. La manière avec laquelle la campagne pour le référendum sur la nouvelle Constitution, votée le $1^{\text {er }}$ novembre 2020, ponctuée notamment par un verrouillage médiatique étouffant toutes les voix discordantes, l'atteste à nouveau.

\section{Bibliographie}

AHCÈNE-DJABALLAH Belkacem, «Économie de la presse et de la communication en Algérie: de l'aventure intellectuelle à la mésaventure économique», NAQD, n 37, 2019. https://doi.org/10.3917/naqd.037.0051

BENCHENNA Abdelfettah, MARCHETTI Dominique, "Écrire entre les "lignes rouges": I'espace de la presse électronique au Maroc et ses enjeux», in Les Industries culturelles et créatives dans la mondialisation, sous la dir. de Bruno Péquignot, Paris, L'Harmattan, 2019. https://hal.archives-ouvertes. $\mathrm{fr} / \mathrm{hal}-02109002 /$

DRIS Chérif, «La presse algérienne: une dérégulation sous contraintes. Les nouvelles formes de contrôle ou la "main invisible" de l'État», Questions de communication, $\mathrm{n}^{\circ}$ 32, 2017.

KLAUS Enrique, "L'autorité de la HAICA sur le secteur tunisien des médias: un anachronisme transitionnel ?», L'Année du Maghreb, n 13, 2015. https:// journals.openedition.org/anneemaghreb/2606 
LES CAHIERS DE LA COMMUNICATION, «Pluralisme, professionnalisme, crédibilité », ministère de la Communication, mai 2015. http://www. ministerecommunication.gov.dz/fr/node/1202

ZOUAIMIA Rachid, "L'autorité de régulation de l'audiovisuel ", Revue académique de la recherche juridique, vol. 9, n 1, 2018. https://www.asjp. cerist.dz/en/downArticle/72/9/1/57746

ZAID Bouziane, "A Normative Study of Broadcast Regulators in the Arab World ", International Journal of Communication, $n^{\circ} 12,2018$. https://ijoc. org/index.php/ijoc/article/download/7001/2495

\section{Sources}

APS, «Le ministère de Communication en passe d'élaborer un projet de loi sur la publicité », Algérie Presse Service, 23 novembre 2020. https://www. aps.dz/algerie/113229-belhimer-le-ministere-en-passe-d-elaborer-unprojet-de-loi-sur-la-publicite

EL KADI Ihsan, «Les jours de malheur d'Abdelmadjid Tebboune», Maghreb Émergent, 5 avril 2020. https://maghrebemergent.info/les-100-jours-demalheur-de-abdelmadjid-tebboune/

GHALI Lila, «Le marché est estimé à 200 millions de dollars: ces 4000 agences de com' qui gèrent la pub !», Algérie 1, 9 décembre 2016. https://www. algerie1.com/actualite/le-marche-est-estime-a-200-millions-de-dollarsces-4-000-agences-de-com-qui-gerent-la-pub

HAMMADI Souhila, "Des éditeurs réagissent: "Le décret est liberticide et techniquement inapplicable" », Liberté, 10 décembre 2020. https://www. liberte-algerie.com/actualite/le-decret-est-liberticide-et-techniquementinapplicable-350492

JORADP, Décret d'application n 11-216 du 10 rajab 1432 (12 juin 2011) fixant les attributions du ministère de la Communication, $\mathrm{n}^{\circ} 33,50^{\mathrm{e}}$ année, 12 juin 2011. http://www.ministerecommunication.gov.dz/sites/default/ files/F2011033\%200RG\%20COM_4.PDF

JORADP, Loi organique $\mathrm{n}^{\circ} 12-05$ du 18 safar 1433 (12 janvier 2012) relative à l'information, $\mathrm{n}^{\circ} 2,5^{\mathrm{e}}$ année, 15 janvier 2012. http://www.joradp.dz/FTP/ JO-FRANCAIS/2012/F2012002.pdf

JORADP, Loi $\mathrm{n}^{\circ}$ 14-04 du 24 rabie ethani 1435 (24 février 2014) relative à I'activité audiovisuelle, $\mathrm{n}^{\circ} 16,53^{\mathrm{e}}$ année, 21 Joumada El Oula 1435 (23 mars 2014). https://amb-algerie.fr/wp-content/uploads/2014/04/ LOI-N\%C2\%B0-14-04-DU-24-FEVRIER-2014-RELATIVE-AL\%E2\%80\%99ACTIVITE-AUDIOVISUELLE.pdf 
JORADP, Décret d'application $n^{\circ} 16-220$ du 8 dhou el kaâda 1437 (11 août 2016) fixant les conditions et les modalités de mise en œuvre de l'appel à candidatures pour l'octroi de l'autorisation de création d'un service de communication audiovisuelle thématique, $\mathrm{n}^{\circ} 46,5^{\mathrm{e}}$ année, 14 dhou el kaâda 1437 (17 août 2016). http://www.ministerecommunication.gov. dz/en/node/2722

JORADP, Décret d'application $n^{\circ}$ 16-221 du 8 dhou el kaâda 1437 (11 août 2016) fixant le montant et les modalités de versement de la contrepartie financière liée à l'autorisation de création d'un service de communication audiovisuelle thématique, $\mathrm{n}^{\circ} 46,5^{\mathrm{e}}$ année, 14 dhou el kaâda 1437 (17 août 2016). http://www.ministerecommunication.gov.dz/en/node/2722

JORADP, Décret d'application $n^{\circ}$ 16-222 du 8 dhou el kaâda 1437 (11 août 2016) portant cahier des charges générales fixant les règles imposables à tout service de diffusion télévisuelle ou de diffusion sonore, $n^{\circ} 46,5^{e}$ année, 14 dhou el kaâda 1437 (17 août 2016). http://www.ministerecommunication. gov.dz/en/node/2722

JORADP, Décret d'application $\mathrm{n}^{\circ}$ 20-332 du 6 rabie ethani 1442 (22 novembre 2020) fixant les modalités d'exercice de l'activité d'information en ligne et la diffusion de mise au point ou rectification sur le site électronique, $\mathrm{n}^{\circ} 70,59^{\mathrm{e}}$ année, 9 rabie ethani 1442 (25 novembre 2020). https://webservices.dz/images/pdf/F2020070.pdf

LACHICHI Mohamed-Chérif, "Couverture des élections législatives: I'ARAV monte au créneau », Liberté, 31 mars 2017. https://www.liberte-algerie. com/actualite/larav-monte-au-creneau-267365/print/1

RABIA Saïd, "Scandal euse gestion de I'Agence national e de publ icité: I es révélations du PDG de l'ANEP», El Watan, 6 août 2020. https://www. elwatan.com/a-la-une/les-revelations-du-pdg-de-lanep-06-08-2020. 


\section{Partie II \\ Les enjeux de la reconfiguration des espaces médiatique, politique et économique}





\title{
Chapitre 4
}

\section{Une figure centrale mais éphémère du journalisme en Égypte: le journaliste du «mouvement social»}

\author{
Bachir Benaziz
}

L'une des conséquences premières du retour du financement privé de la presse écrite en Égypte et le développement des protestations collectives au cours des années 2000 est l'émergence d'une nouvelle figure du «journalisme-justicier» (Lemieux, 2001; Benaziz, 2021), incarné par des rédacteurs chargés de couvrir les mouvements sociaux. Figure centrale du paysage médiatique égyptien des années 2000 , elle ne débouchera pas pour autant sur la formation d'un groupe professionnel intéressé par la promotion du «mouvement social» comme catégorie à part entière et légitime d'intervention journalistique. Malgré son importance objective pour un pays comme l'Égypte et son potentiel critique à l'égard du pouvoir politique, la "question sociale», entendue ici comme "une catégorie pertinente de description de la réalité» (Lévêque, 2000), ne bénéficie par ailleurs dans la presse égyptienne d'aucune page ou rubrique spécifique où pourraient être réunies, comme c'est le cas par exemple dans la presse écrite arabophone au Maroc, des problématiques en lien avec la santé, l'éducation, le transport ou l'habitat. Dans un champ journalistique déjà faiblement spécialisé, le traitement de ce type de sujet est le plus souvent, s'il n'est pas censuré, dispersé entre les différents services qui organisent le travail des rédactions égyptiennes. Les années 2000 constituent de ce fait une parenthèse notoire dans I'histoire de la presse écrite du pays puisque, pour la première fois, des rédacteurs vont être chargés de couvrir, de façon régulière et intense, des mouvements de revendications sociales et politiques. Ainsi, ils participent non seulement à la médiatisation de ces dernières et des acteurs qui les portent, mais également à la réussite fulgurante des premiers journaux 
privés, qui se sont rapidement saisis du «mouvement social » comme une opportunité de distinction et de légitimation professionnelles.

Comment expliquer toutefois le caractère éphémère de cette nouvelle figure du "journalisme-justicier » ? II est très significatif qu'avant même le coup d'État militaire de juillet 2013 et le durcissement de la répression policière, quasiment aucun des principaux journalistes qui s'étaient distingués durant «l'ancien régime» par la couverture des mouvements sociaux ne signait de papier sur le sujet. Au contraire, c'est au moment même où les conditions de production de l'information s'étaient relativement améliorées, en comparaison avec les années Moubarak, que la plupart des représentants de ce type de journalisme avaient cessé d'écrire sur les mouvements sociaux. À travers les biographies professionnelles des journalistes qui ont fortement couvert les mouvements de protestation ou qui ont participé à la promotion de ce type de sujets en Égypte ${ }^{1}$, ce chapitre montre que l'invention et le développement d'un genre journalistique, a fortiori dans un système autoritaire où la contrainte politique pèse lourdement sur les pratiques du métier, sont indissociables du lieu de production journalistique, ici le «journal privé », qui rend possible ou impossible à un moment donné l'expression de tel ou tel type de journalisme.

\section{Presse d'information et mouvement social: le cas du quotidien Al-Masry Al-Youm (L'Égyptien aujourd'hui)}

Dans l'ensemble, et pour ne retenir ici que les journalistes qui ont réussi leur insertion professionnelle, le passage par la couverture des mouvements sociaux a été un moyen pour des jeunes rédacteurs non encartés, sans expérience et ayant très peu accès aux instances officielles de s'imposer rapidement dans le champ journalistique. C'est à travers la valorisation du terrain et des sources d'informations considérées, historiquement, comme "secondaires » ou «illégitimes», tels les chercheurs et les militants syndicaux, qu'ils se sont distingués de leurs consœurs et confrères. Comme le souligne une journaliste chargée de couvrir les mouvements de jeunes pour un journal privé entre 2008 et $2012^{2}$, cette politique visant à engager

\footnotetext{
${ }^{1}$ Ce travail est issu d'un chapitre de ma thèse, soutenue en 2018. L'enquête s'appuie, notamment, sur une vingtaine d'entretiens semi-directifs effectués avec des journalistes concernés par la couverture les mouvements sociaux durant les années 2000.
}

${ }^{2}$ Entretien avec une journaliste, Le Caire, 2013. 
des jeunes diplômés, le plus souvent sans contrat de travail, correspondait à des logiques managériales très répandues alors au sein de la presse écrite égyptienne. II s'agissait de recruter "des jeunes étudiants, ambitieux et sans expérience, donc capables de travailler dans conditions financières et professionnelles précaires, mais aussi ayant la capacité d'être sur le terrain à longueur de journée ${ }^{3}$. Dans le cadre de nouvelles entreprises de presse où la majeure partie des rédacteurs sont des jeunes diplômés et face au monopole ou le quasi-monopole qu'exerçaient alors les quotidiens de I'État, notamment Al-Ahrâm, sur "l'information officielle», I'apprentissage du métier et la distinction professionnelle vont s'exprimer par la capacité du rédacteur à s'investir sur des problématiques marginalisées par les médias officiels.

La chute du régime Moubarak au mois de février 2011 et l'engagement d'un processus dit de «transition démocratique» ont eu deux effets notoires: d'une part, les journaux privés, dont le nombre augmenta également, furent moins sensibles à focaliser l'attention médiatique ou à construire la distinction éditoriale sur les mouvements sociaux; d'autre part, les compétences spécifiques de ce type de journalisme, qui se caractérisait essentiellement au temps de Moubarak par un certain "courage » ou «prise de risque» dans la couverture de mouvements anti-régime, se désingularisèrent. Parallèlement, le «retour du politique» et l'« ouverture démocratique " consécutifs au départ de Moubarak, la naissance de nouveaux partis plus influents (notamment islamistes), la multiplication des échéances électorales et la création de nouveaux ministères chargés de prendre en compte les revendications sociales soulevées lors des événements du 25 janvier 2011 ont conduit à un regain d'intérêt des journaux égyptiens pour la couverture de la vie politique instituée (Steuer, 2012).

Aussi, dès les premiers mois de la révolution, les protestations collectives à caractère social avaient quasiment disparu des pages des principaux quotidiens d'information politique et générale. À ce titre, le cas du quotidien Al-Masry Al-Youm (L'Égyptien aujourd'hui) est très significatif. Ce dernier, fondé en 2004 par l'homme d'affaires Salah Diab et l'éditeur Hisham Kassem, est de loin le quotidien qui a le plus écrit sur les protestations sociales ou salariales des années 2000. II est également le seul journal, parmi ceux qui ont fortement couvert les mouvements sociaux, à avoir survécu à la censure 
du régime de Moubarak. Le nombre d'articles consacrés à ce sujet s'élevait, en effet, à plus de 1100 entre 2006 et 2008, contre seulement 200 entre 2012 et 2014. Plus significatif encore, les «mobilisations collectives» font partie des thématiques qui ont le plus fait la «Une» du journal entre janvier 2006 et décembre 2007. Ces chiffres traduisaient, au temps de Moubarak, une stratégie éditoriale consistant à construire l'actualité médiatique du pays, entre autres autour du «mouvement social» et, plus globalement, sur la valorisation d'acteurs et de problématiques non traités par les médias du régime. Le quotidien Al-Masry Al-Youm n'avait par ailleurs, durant ses premières années, aucun journaliste accrédité auprès de l'institution présidentielle, ni auprès du conseil des ministres, ni auprès de certains ministères-clés du régime de Moubarak.

"On a eu beaucoup de problèmes dans notre travail, et dès le premier jour. On a été le journal à qui on retardait l'autorisation pour ses journalistes auprès de certains secteurs sensibles. On nous a refusé beaucoup de demandes pour couvrir certaines sources. En Égypte, nous avons des ministères de services et des ministères de gouvernement, après il y le conseil des ministres, le ministère de l'Intérieur, le ministère de la Défense, et enfin on a la présidence de la République. Ça c'est le classement normal, en termes de difficulté, pour avoir un journaliste accrédité (mo'tamad) auprès de ces sources. Plus vous montez, plus ça se complique. Durant les premières années du journal, on avait un problème pour avoir un représentant auprès du conseil des ministres. À chaque fois ils disaient qu'ils examinaient le dossier de notre journaliste, puis, nous, on ripostait violemment, alors ils essayaient de nous « attirer». Plusieurs fois le porte-parole du conseil des ministres envoyait des messages à l'éditeur ou aux actionnaires, nous demandant de calmer le jeu pour pouvoir accréditer nos représentants. On calmait le jeu, mais peu de temps après, dès qu'un grand événement survenait, on " achetait l'événement» et on oubliait le représentant, et on perdait alors I'accréditation. Le premier journaliste accrédité qu'on a eu auprès du conseil des ministres, c'était en 2009. Entretemps, nous, on travaillait à travers des collègues des journaux du gouvernement et qui écrivaient avec des noms d'emprunt. Cela s'est répété avec plus de sévérité encore avec l'institution présidentielle. On n'a jamais pu avoir de journaliste accrédité auprès de la présidence de la République du temps de Moubarak.» (Entretien avec I'ancien rédacteur en chef du quotidien Al-Masry Al-Youm, Mohammed Al-Sayyed Saleh, qui fut responsable pendant plusieurs années du service de l'information au sein du quotidien, Le Caire, 2014, traduit par l'auteur)

Pour faire face à la concurrence des quotidiens de l'État et surmonter les contraintes instaurées sur la couverture de certaines institutions et 
ministères-clés, les fondateurs d'Al-Masry Al-Youm avaient développé plusieurs stratégies qui allaient progressivement construire l'identité du journal et assurer son influence. La première consistait à ne pas circonscrire la production journalistique pour le seul lectorat du Caire. Grâce à l'établissement d'un important réseau de correspondants, Al-Masry Al-Youm élargit son champ de couverture aux autres gouvernorats afin de se démarquer d'une presse écrite égyptienne globalement très centrée sur la capitale. En l'absence également d'une presse régionale forte et influente, l'attention du journal à ce qui se passait en dehors de la région du Caire, le centre politique et économique du pays, constituait un véritable marqueur de distinction et de crédibilité pour le quotidien dont la devise est le «droit de savoir ». Contrairement ainsi à la plupart des journaux égyptiens, les articles concernant les gouvernorats, "al-mohafazat», ne sont pas réunis dans une page spécifique ou tout simplement ignorés, mais intégrés dans les différentes rubriques qui organisent le traitement de l'actualité. Cette mise en avant d'informations ou d'« histoires » en provenance de la province par un quotidien cairote aura pour conséquence, en autre autres, de nationaliser les problématiques régionales et dont la plupart portent sur les préoccupations économiques et sociales des populations locales. L'analyse des numéros du quotidien Al-Masry Al-Youm révèle ainsi une attention forte sur tout ce qui relève des problèmes d'infrastructure, de logement et de santé, ou des affaires de corruption.

La deuxième stratégie concerne la notion même de sources d'informations dites "officielles», une notion "fourre-tout» (Schlesinger, 1992) et dont les journalistes fondateurs du quotidien semblent avoir bien saisi l'impertinence. En effet, pas tous les hommes politiques, ministres, responsables administratifs et autres structures qui forment l'appareil de l'État égyptien ne bénéficient du même intérêt de la part de la presse "officielle». Si le président Moubarak, les cadres du parti au pouvoir et le conseil des ministres occupent une place de choix dans les pages d'un quotidien comme Al-Ahrâm, il existe des secteurs entiers de l'administration et de la vie politique égyptienne qui ont très peu accès, voire aucun, au système de production de l'information. Il y a donc des "sources" marginalisées ou déconsidérées à l'intérieur même du «camp officiel » et que la rédaction du journal Al-Masry Al-Youm va s'efforcer de mettre en avant. C'est ainsi le cas de l'administration des régions, des ministères de services, 
des syndicats professionnels, mais surtout de l'institution législative ${ }^{4}$. Une autre stratégie du journal repose sur la valorisation des sources d'information dites "non officielles» ou «illégitimes»: les mouvements sociaux, les forces politiques de l'opposition, les centres de recherche, les Frères musulmans, les associations de droits de l'homme, les activistes, les universitaires, les leaders syndicaux, etc.

Dans le cadre d'une ligne éditoriale qui se veut «libérale et réformatrice», le but du journal est de révéler des enjeux politiques et sociaux capables de susciter un mouvement de débat public, en amplifiant la parole de groupes sociaux généralement incapables de se faire entendre à travers les médias du régime. Mais il ne s'agit pas ici, comme le précise l'ancien rédacteur en chef du quotidien Al-Masry Al-Youm, Anouar El-Hawary, « de faire du journal une brochure de doléances, mais de faire de ces doléances une information ${ }^{5}{ }$. Concrètement, cela passe par une certaine froideur et neutralité dans l'écriture des articles et la formulation des titres, une objectivité dans le traitement de l'actualité et la transformation des problèmes en enquêtes et une mise en page valorisante qui donne plus d'importance à la fermeture d'une école primaire en province qu'aux déclarations de tel ou tel membre du parti au pouvoir.

La réinvention de la Une peut être considérée comme l'une des œuvres principales des fondateurs du Masry Al-Youm, grâce notamment au travail du journaliste Ahmad Mahmoud, directeur technique et concepteur de I'identité visuelle du journal. Contrairement à la plupart des journaux de l'époque où le lecteur se perd facilement dans une première page surchargée de titres, Al-Masry Al-Youm se démarque par une mise en forme aérée, attrayante, centrée sur une manchette en gros caractères et appuyée par une photo. L'analyse des manchettes du quotidien entre le 1er janvier 2006 et le 31 décembre 2007, sur un total de 668 numéros, met en évidence l'intérêt du journal pour la valorisation de thématiques non traitées par les médias du régime.

\footnotetext{
${ }^{4}$ Les débats au sein du parlement égyptien représentent avec les mouvements de protestation un des thèmes qui ont le plus fait la Une du Masry Al-Youm au milieu des années 2000. La percée historique des Frères musulmans et des candidats indépendants aux législatives de 2005 a été particulièrement providentielle pour le nouveau journal. Les débats houleux entre les députés de l'opposition et ceux du parti au pouvoir ont permis au quotidien d'axer l'actualité politique égyptienne sur l'institution parlementaire.

${ }^{5}$ Entretien du journaliste Anouar Al-Hawary avec la rédaction du journal Al-Masry Al-Youm dans le cadre d'un dossier spécial consacré au quotidien, le 22 juin 2014.
} 
Tableau 1

Les thèmes des manchettes $(n=668)$ du quotidien Al-Masry Al-Youm entre le $1^{\text {er }}$ janvier 2006 et le 31 décembre 2007

\begin{tabular}{|l|c|c|c|}
\hline Thème & $\mathbf{2 0 0 6}$ & $\mathbf{2 0 0 7}$ & Total \\
\hline Mouvements de protestation & 30 & 44 & 74 \\
\hline Gouvernorats & 13 & 19 & 32 \\
\hline Société $^{6}$ & 18 & 27 & 45 \\
\hline Économie & 3 & 11 & 14 \\
\hline Droits et libertés & 16 & 23 & 39 \\
\hline International & 58 & 42 & 100 \\
\hline Parlement & 40 & 37 & 77 \\
\hline Justice & 41 & 14 & 55 \\
\hline Politique & 26 & 35 & 61 \\
\hline Moubarak & 8 & 7 & 15 \\
\hline Parti au pouvoir & 7 & 6 & 13 \\
\hline Gamal Moubarak & 2 & 1 & 3 \\
\hline Mouvements islamistes & 19 & 42 & 61 \\
\hline Partis de l'opposition autorisés & 8 & 6 & 14 \\
\hline Ministères de services & 12 & 14 & 26 \\
\hline Conseil des ministres $^{7}$ & 9 & 3 & 12 \\
\hline Syndicats & 4 & 4 & 8 \\
\hline Autres & & 7 & 19 \\
\hline
\end{tabular}

Ce système de production de I'information du journal Al-Masry Al-Youm s'appuie aussi dès le départ sur une organisation du travail par "secteur» ou par "dossier», chaque rédacteur étant responsable d'une problématique dans sa globalité et non pas, comme c'est le cas au sein de la presse étatique, limité à une "couverture de ministère ${ }^{9}$ ». L'imbrication des institutions journalistiques «nationales» avec les divers appareils de l'État a joué en effet un rôle fondamental dans la définition du journalisme

\footnotetext{
${ }^{6}$ Il s'agit ici d'articles qui portent principalement sur des affaires de religion, des problèmes de logement, de santé et des questions liées à l'enseignement.

${ }^{7}$ Cette case englobe en fait tous les articles à caractère « politique » qui ne portent sur aucun groupe ou thématique répertoriés ici beaucoup concernent des affaires de corruption de responsables politiques et des articles sur l'amendement constitutionnel de 2007.

${ }^{8}$ II s'agit ici, pour la plupart, d'articles liés aux faits divers et au sport.

${ }^{9}$ Entretien avec Charles Al-Masry, directeur exécutif du journal Al-Masry Al-Youm, Le Caire, 2013.
} 
en Égypte. Jusqu'à l'arrivée de la presse partisane et privée, l'univers journalistique était profondément structuré par le fonctionnement, les politiques et la communication du régime. L'obligation constante dans laquelle se trouvaient les quotidiens de l'État de privilégier, voire de limiter, la production de l'information aux déclarations et rapports livrés par les responsables politiques et administratifs a rendu les journalistes de ces structures entièrement dépendants des sources d'information officielles. La distinction professionnelle se jugeait ainsi non pas sur la capacité du journaliste à soulever des problématiques neuves ou des «affaires» ignorées, mais sur sa possession, parfois héréditaire (Hamamou, 2012), du «droit » d'écrire sur un ministère ou une institution étatique quelconque et dont il s'impose de restituer fidèlement les activités. Comme l'exprime bien une journaliste qui a couvert les mouvements sociaux entre 2006 et 2011 : «Dans la presse étatique, l'idée de scoop n'existe pas ${ }^{10}$.»

La renaissance en Égypte de la presse d'information, à travers le quotidien Al-Masry Al-Youm, a eu des effets politiques et sociaux redoutables pour le régime de Moubarak. L'éloignement du journalisme d'opposition, la valorisation du travail d'enquête, la stricte observation des faits et la recherche permanente de l'objectivité vont permettre ainsi au journal de gagner en crédibilité et d'accompagner la montée des protestations collectives des années 2000. Le journal a ainsi été fortement présent dans la couverture de tous les mouvements sociaux qui ont marqué cette période, notamment ceux porteurs de revendications sociales ou salariales. Globalement, le dépouillement des archives du quotidien Al-Masry Al-Youm entre janvier 2006 et décembre 2008 montre une généralisation de la couverture journalistique à toutes sortes de mobilisations collectives: sit-in, grèves, manifestations, blocages de routes, etc. Soit la place accordée à l'événement se réduit à quelques lignes, soit il peut faire l'objet de tout un dossier et s'étaler sur plusieurs pages, voire plusieurs numéros, mais la couverture reste systématique et régulière. Cet intérêt du journal pour l'action collective s'expliquait, d'abord et avant tout, par les conceptions journalistiques défendues par les fondateurs du quotidien et les conditions de production de l'information sous Moubarak. Ces dernières avaient amené le journal à investir intensément dans ce type de sujet. Cet intérêt s'expliquait également par le travail des correspondants qui focalisaient l'attention sur les difficultés sociales et économiques des populations des régions. Ceci conduisait à donner une place importante à toute forme d'action collective pouvant susciter rapidement la réaction du

${ }^{10}$ Entretien avec une journaliste, Le Caire, 2014. 
pouvoir politique central. Les articles des correspondants qui soulèvent des problèmes de logement, d'infrastructure, de pollution ou d'accès à l'énergie étaient en effet très fréquents. Or, une grande partie des protestations sociales que nous avons recensées portent sur ce type de problématique.

Après 2011, l'ouverture relative du jeu politique va s'accompagner d'une ouverture des sources d'information officielles face aux journalistes de la presse privée, entraînant ce que l'on pourrait qualifier de «ministérialisation » de la question sociale en Égypte. Désormais, le traitement journalistique des protestations sociales ou salariales, qui disparaissent rapidement des pages du quotidien Al-Masry Al-Youm, est remplacé par la couverture des ministères dits "sociaux» des différents gouvernements post-Moubarak. La trajectoire du journaliste Mohammed Azouz, aujourd'hui responsable du "service des informations » pour le quotidien Al-Masry Al-Youm, est très révélatrice de ces déplacements et qui touche par ailleurs tout le champ journalistique égyptien. Diplômé de sociologie en 2003, Mohammed Azouz intègre le quotidien Al-Masry Al-Youm dès sa fondation. Entre 2005 et 2011, il est chargé de «tout le dossier sur les mouvements de protestation, et plus particulièrement les protestations ouvrières ${ }^{11} »$. Interrogé sur les conditions de travail et les possibles censures, internes ou externes, qui seraient liées à la couverture des mouvements sociaux, le journaliste affirme n'avoir jamais subi aucune forme de pression ou de restriction hiérarchique. Au contraire, selon lui, la direction était très élogieuse à l'égard de son travail, et les papiers sur les mouvements sociaux étaient très souvent prioritaires. Moins célèbre à l'époque que l'ancien journaliste du Dustûr, Mostapha Bassiouny, qui couvrait les mêmes sujets, il reste pour autant le journaliste ayant le plus écrit sur les mobilisations des travailleurs. Sur les 1080 numéros que nous avons étudiés, environ 1890 textes journalistiques portent sur les «mouvements sociaux», quasiment tous apparaissent dans les deux rubriques principales du quotidien : les «Dossiers chauds » et les «Infos de la nation » ${ }^{12}$. La plupart sont des comptes-rendus d'actions collectives écrits par le journaliste Mohammad Azouz ou les correspondants d'Al-Masry Al-Youm ${ }^{13}$.

\footnotetext{
${ }^{11}$ Entretien avec le journaliste Mohammad Azouz au siège du quotidien Al-Masry Al-Youm, Le Caire, 2014 et 2015.

${ }^{12}$ Sur les seize pages du quotidien Al-Masry Al-Youm, les rubriques principales et régulières qui organisent la production du journal sont les «Dossiers chauds», "Infos de la nation», "Économie», "Sport», "Arts», "Faits divers», "Les infos du monde», ainsi que les pages réservées aux articles d'opinion.

${ }^{13}$ Outre les comptes rendus d'actions collectives, le corpus journalistique étudié comprend également des reportages, la publication des rapports de la Cour des comptes et des centres
} 
N'étant affilié à aucun parti ou mouvement, Mohammed Azouz est probablement le rédacteur-type recherché alors par la rédaction. Malgré les passions et prises de position que peut soulever la couverture des protestations sociales, ses écrits révèlent un journaliste qui trouve "une réelle satisfaction professionnelle à rester neutre ${ }^{14}$ ». Le "récit d'information », "al-khissat al-khabariyyât», qui est la mise en forme narrative élaborée progressivement par le quotidien pour répondre aux exigences éditoriales d'objectivité et de sacralité de l'information, semble donc parfaitement en affinité avec sa personnalité et sa formation en sociologie. Si la plupart de ses papiers entre 2006 et 2011 portent sur les protestations salariales, le journaliste veille à ce que toutes les parties en conflit soient représentées. Outre les ouvriers ou employés, on retrouve aussi, pour ne citer que les groupes d'acteurs qui reviennent le plus souvent dans ses écrits, des responsables politiques et administratifs, des représentants syndicaux, des députés, des présidents de centres et associations concernés par la défense des droits des travailleurs, ainsi que des dirigeants d'entreprises publiques. L'idée défendue par Mohammad Azouz consiste en effet à ne pas favoriser un camp sur un autre, mais à «transposer sur le papier l'état de lutte» qui existe alors en Égypte sur le plan social ${ }^{15}$. Après le départ de Moubarak en février 2011 et la formation du premier gouvernement dit de "transition», Mohammed Azouz est chargé de couvrir les nouveaux ministères de la Solidarité et du Commerce extérieur et celui des Assurances et des Affaires sociales, censés répondre aux revendications socioéconomiques des révolutionnaires. II s'agit là d'une promotion. Mais ce qui est alors considéré comme une "progression professionnelle et personnelle ${ }^{16}$ » atteste plus profondément, d'une part, d'un processus d'étatisation officieuse de journal Al-Masry Al-Youm, processus que la restauration autoritaire n'a fait en vérité qu'accélérer, marqué par la volonté permanente des propriétaires, dès 2012, de mettre à la tête du journal des personnalités issues des institutions médiatiques de l'État et proches du pouvoir; d'autre part, cette trajectoire professionnelle témoigne de la difficulté de préserver le modèle journalistique et institutionnel établi sous la direction de l'éditeur Hicham Kassem durant les années 2000. La

de droits de l'homme, ainsi que des entretiens effectués avec des responsables politiques et des syndicalistes.

${ }^{14}$ Entretien avec le journaliste Mohammad Azouz au siège du quotidien Al-Masry Al-Youm, Le Caire, 2014 et 2015.

$15 \mathrm{lbid}$.

16 lbid. 
situation révolutionnaire et le gouvernement de la confrérie conduisent en effet le journal à glisser tout d'abord dans l'engagement politique, avant de chercher à renouer avec modèle informationnel une fois l'autorité politique du président El-Sissi consolidée. Signe d'une crise identitaire qui perdure, une multitude de rédacteurs en chef et plusieurs tentatives de restructuration se succèdent entre 2012 et 2018 au sein du quotidien Al-Masry Al-Youm. Par exemple, dirigée en 2016 par l'éditeur Hicham Kassem et accompagnée d'un licenciement massif de rédacteurs, la restructuration avait pour objectif de ramener le quotidien aux «fondamentaux». Mais dans un contexte autoritaire très répressif, le journal finit par se retrancher dans un neutralisme inoffensif, jouant le rôle d'une machine quasi officielle de l'État où prédominent les nouvelles sur l'armée et l'action du président, mais sans l'influence ni la dimension libérale d'autrefois. La dernière fois où les convictions journalistiques du quotidien furent mises à l'épreuve, c'était lors des élections présidentielles de 2018. L'enquête du journal Al-Masry Al-Youm, qui parlait alors de "pressions de l'État pour inciter les gens à voter», avait conduit à la censure pure et simple du numéro, la démission du rédacteur en chef, Mohammed Al-Sayeed Saleh et son remplacement par un journaliste du régime, Hamdy Rizk.

\section{Journal révolutionnaire et revanche sociale}

II serait pour autant réducteur de ramener l'apparition - et la disparition - de cette nouvelle figure du «journalisme-justicier» en Égypte aux seules logiques de l'ambition professionnelle et de l'opportunisme éditorial. L'idée d'une «revanche sociale» sur les élites gouvernantes doit être également mobilisée. Le recrutement dans les journaux privés fondés entre 2004 et 2010 est ainsi marqué par l'arrivée d'une nouvelle cohorte de journalistes issus pour la plupart de catégories sociales relativement défavorisées, notamment celle des "fonctionnaires». Cette catégorie socio-professionnelle a subi durant l'ère Moubarak (1981-2011) une violente paupérisation à la suite des politiques dites d'ajustement structurel. Dans un contexte marqué par une dégradation sensible du pouvoir d'achat des classes populaires et par un climat de forte inquiétude au sein des travailleurs, généré par l'intensification des politiques de privatisation des entreprises publiques sous le gouvernement d'Ahmed Nazif (Duboc, 2012), l'Égypte entame à partir des élections syndicales de novembre 2006 un des plus importants cycles de mobilisations sociales de son histoire récente. 
Une étude du Centre d'études politiques et stratégiques d'Al-Ahrâm, un centre de recherche public, recense à ce titre un total de 2838 actions collectives entre le $1^{\text {er }}$ janvier 2005 et le 31 décembre 2010, étendues à toutes les régions de l'Égypte et partagées notamment entre les secteurs de I'industrie et des services (Al-Azbaoui, 2011). Or, ce sont bien les milieux des ouvriers et des employés de la fonction publique qui forment le moteur des protestations salariales des années 2000, incarnées par deux mouvements emblématiques de cette période: le mouvement des ouvriers de la ville de Mahalla Al-Kûbra (Duboc, 2012) et celui des «fonctionnaires de l'impôt foncier» (Abdallah Iskandar, 2014). À I'heure de la fondation des premiers journaux privés, une nouvelle génération de journalistes égyptiens est donc particulièrement prédisposée à couvrir et à soutenir les mouvements de protestation. La plupart de ces nouveaux entrants vont chercher dans le journalisme les fonctions de «réparateur d'injustices » et de plaidoyer pour la cause de catégories sociales jugées opprimées et non représentées dans les médias officiels. Travailler pour des journaux privés comme Al-Dustûr (La Constitution, 2005-2010) ou Al-Badîl (L'Alternative), dont les lignes éditoriales se voulaient ouvertement "révolutionnaires » et "anti-régime», offrait à la fois la promesse de réussite dans le métier, mais également la possibilité d'une contestation radicale contre l'ordre établi. Les parcours professionnels de deux anciens journalistes d'Al-Dustûr et Al-Badîl, Mostapha Bassiouny, rendu célèbre pour sa couverture des protestations sociales des années 2000, et Khaled El-Balchy, figure emblématique de la presse égyptienne, sont à ce titre exemplaires.

\section{Mostapha Bassiouny et la couverture du mouvement des travailleurs}

Jusqu'à son recrutement par I'hebdomadaire Al-Dustûr en 2005, Mostapha Bassiouny n'avait jamais travaillé pour un journal «autorisé ». Militant au sein du mouvement «Les socialistes révolutionnaires» et diplômé d'une formation au journalisme au milieu des années 1990, son activité dans la presse se limite alors principalement à écrire pour des publications clandestines concernées par la question sociale, lui permettant néanmoins de "construire un réseau très large de relations au sein du «Mouvement des travailleurs égyptiens» («Al-harakat al-'oummaliyyat») $)^{17}$. C'est dans ce

\footnotetext{
${ }^{17}$ Entretien avec le journaliste Mostapha Bassiouny au siège de la maison d'édition étatique Dar al-Hilal, Le Caire, 2014.
} 
cadre qu'il rejoint I'hebdomadaire Al-Dustûr, alors en pleine reconstruction ${ }^{18}$. Incarné par la figure emblématique et contestataire d'Ibrahim Eissa (Benaziz, 2021), fondé dans le contexte des mobilisations politiques anti-Moubarak, le projet Al-Dustûr marque en 2005 la volonté de dépassement des clivages politiques traditionnels derrière la dénonciation du «despotisme», l'urgence de procéder à des réformes démocratiques réelles et la défense de certains idéaux tels que la dignité humaine, la liberté d'expression et la justice sociale. Le journal représente également pour ses fondateurs l'opportunité de produire une expérience journalistique novatrice, en même temps que la possibilité d'écrire sur des problématiques et des catégories sociales délaissées par la presse étatique et partisane. Mostapha Bassiouny est alors chargé de couvrir le milieu ouvrier, et il est l'un des rares rédacteurs du journal à être chargé d'un dossier spécifique. Le succès éditorial fulgurant de ce dernier et ses méthodes de travail vont permettre ainsi à Mostapha Bassiouny d'offrir au milieu ouvrier une couverture journalistique jusqu'alors inédite. Ses publications sont toutefois très rares au début et ne touchent qu'indirectement à la question sociale. Le journal est alors hebdomadaire, et l'espace de rédaction réservé au "service des enquêtes» est investi par l'actualité politique de l'époque: élections présidentielles et législatives de 2005, manifestations du mouvement Kifayat ! (ça suffit !), mouvements des magistrats pour l'indépendance du pouvoir judiciaire (Maugiron, 2007) ainsi que les articles permanents sur la famille Moubarak et le projet de la «transmission héréditaire du pouvoir» (al-tawrith). Ce n'est qu'à partir du $51^{\mathrm{e}}$ numéro du journal que Mostapha Bassiouny parvient à publier un article en lien avec les problèmes des travailleurs (Al-Dustûr, 8 mars 2006). L'enquête révèle la corruption présumée qui a accompagné la privatisation des entreprises publiques et les risques de grèves imminentes dans certains sites industriels où "la conscience syndicale est très forte » (ibid.). Le journaliste rapporte à travers cette enquête la forte inquiétude qui règne au sein du milieu ouvrier face à l'accélération des politiques libérales du régime Moubarak et annonce les protestations salariales à venir. Mais c'est indéniablement avec la publication de deux reportages sur les villes ouvrières de Kafr Al-Dawar et Mahalla Al-Kûbra, en juin et juillet 2006, que Mostapha Bassiouny réussit à lancer sa carrière et situer la «question sociale» au cœur de l'actualité médiatique des années 2000 (Al-Dustûr, 14 juin et 26 juillet 2006). Le lecteur découvre dans ces reportages l'effondrement ou la «lente agonie» des villes industrielles historiques,

${ }^{18}$ Le journal Al-Dustûr a connu une première expérience entre 1995 et 1998 (El-Khawaga, 2001). 
connues notamment pour la production de textile, bâties dans les années 1930 et 1940, gagnées par le "chômage», la «misère», le "pillage» et la «mauvaise gestion» (ibid.). C'est moins la dimension informationnelle qui caractérise ces papiers que les formes journalistiques utilisées pour «raconter la visite du Dustûr» à Mahalla Al-Kûbra et à Kafr Al-Dawar. Avec un style accrocheur, agréable à lire et accompagné des dessins des caricaturistes Walid Taher et Mohamed Makhlouf, Mostapha Bassiouny décrit l'abandon de la politique économique de «l'industrie nationale» sous Moubarak et ses conséquences sur les populations des villes industrielles. Tous les aspects de la dégradation des conditions de vie des ouvriers sont ainsi dépeints: érosion des salaires, effondrement des infrastructures et des services de santé, corruption des directions, féminisation de la maind'œuvre (car moins chère), disparition des espaces de divertissement pour les ouvriers, logements étroits et insalubres où les gens sont «entassés comme dans des boîtes de sardines», d'autres "transformés en tombes», contrairement à ceux réservés à la direction, aux cadres de l'entreprise et aux officiers de la Sécurité de l'État (ibid.).

Les publications de Mostapha Bassiouny deviennent par la suite plus régulières, multiples et fort sollicitées par la direction du journal qui décide en 2007 de faire du mouvement social sa signature ${ }^{19}$. D'autant plus que l'activisme du journaliste et ses rapports privilégiés avec les leaders des mouvements ouvriers conduisent à situer le journal au cœur du processus protestataire. Le traitement de la «question sociale» par Mostapha Bassiouny va épouser ainsi les formats journalistiques qui ont assuré le succès du journal avec les mouvements de revendications politiques: enquêtes, reportages, satires et caricatures, organisations de conférences au sein du journal, entretiens avec des leaders syndicaux et des économistes, publication de rapports de centres de recherches. Ces articles touchent à peu près toutes les dimensions: salaires, inégalités sociales, chômage, grèves, représentation syndicale des travailleurs et enquêtes sur les entreprises publiques. Du coup, quand éclate la grève ouvrière du complexe de textile et de tissage de Ghazl Al-Mahalla en décembre 2006, Mostapha Bassiouny est à Mahalla Al-Kûbra où 27000 ouvriers protestent contre la médiocrité de leurs salaires et les conditions de travail. D'après Kamal 'Abbas, fondateur et coordinateur du Centre de services pour les syndicats et les ouvriers (Dar al-khadamat al-nakhabiyyat wa al-'ummaliyyat), la mobilisation de Ghazl Al-Mahalla en décembre 2006 constitue un tournant dans I'histoire

${ }^{19}$ Entretien avec l'ancien directeur exécutif du journal Al-Dustûr, Le Caire, 2013. 
contemporaine des protestations ouvrières en Égypte (Entretien, 2014). Outre la médiatisation inédite dont l'action des grévistes avait bénéficié, le recours même à la grève comme modalité d'action protestataire constituait une nouveauté. Pour réclamer des augmentations de salaire ou le paiement de primes, l'action des ouvriers consistait le plus souvent à organiser des sit-in, durement et rapidement réprimés par les forces du régime, au cours desquels la production n'arrêtait pas, voire augmentait, afin de signifier le caractère pacifique de la mobilisation et la légitimité des revendications. À partir de la grève de Ghazl Al-Mahalla en décembre 2006, qui va durer trois jours et au terme de laquelle les travailleurs vont obtenir gain de cause, ce mode d'action devient le mécanisme central et principal des protestations ouvrières et prend souvent plusieurs jours avant la résolution de la crise. L'allongement du temps de la mobilisation, l'absence de réaction répressive de la part des forces de l'ordre et l'ouverture d'un espace de négociation entre les grévistes et l'État ont permis l'émergence d'une nouvelle forme de traitement journalistique des protestations collectives, marquée par une immersion intensive du journaliste dans la couverture de l'événement. Mostapha Bassiouny propose ainsi un journalisme de terrain qui raconte et décrit "la vie des ouvriers en mouvement». Si le parti-pris du journaliste pour les grévistes est manifeste, il est compensé par la richesse des descriptions (déroulement de la mobilisation, conduite des négociations, gestion du sit-in, etc.), par la diversité des témoignages recueillis sur place et le style d'écriture qui témoigne du souci du journaliste de partager avec le lecteur les «choses vues».

«Pour moi il n'y a aucune contradiction entre mon travail de journaliste et mes préoccupations ou mon orientation politique. En fin de compte, mon devoir en tant que journaliste est de révéler la vérité. Politiquement parlant, j'ai un rôle, celui d'être solidaire et interactif avec le mouvement des travailleurs. II n'y avait pas de contradiction, au contraire, mon interaction avec le mouvement des travailleurs m'a fait découvrir des choses que la plupart des journalistes concernés par la question sociale ignoraient, et c'était un point fort pour moi et non un défaut. Par exemple, lors de la grève de Mahalla, parce que je "vivais la grève" depuis le début jusqu'à la fin, j'ai pu découvrir beaucoup de choses. La grève se passait au mois de ramadan, j'ai pu rapporter comment les ouvriers géraient leurs besoins quotidiens en termes d'alimentation et tout le reste, car il y avait beaucoup de rumeurs qui disaient que les ouvriers étaient financés par certaines forces politiques pour pouvoir continuer la grève. Donc j'ai pu prouver, photos à l'appui, que les ouvriers se débrouillaient par eux-mêmes, qu'ils ramenaient la nourriture de leurs maisons et que c'est ce qu'ils mangeaient habituellement. Tout 
ça était utile pour la presse et utile aussi pour le mouvement.» (Entretien avec le journaliste Mostapha Bassiouny, Le Caire, 2014, traduit par l'auteur)

La couverture des grèves et sit-in, qui deviennent de plus en plus longs après la réussite de la grève du complexe industriel de Ghazl Al-Mahalla, est l'occasion pour le journaliste Mostapha Bassiouny de produire des papiers où la «condition ouvrière » en Égypte se dévoile en action, permettant ainsi de faire connaître des problématiques et des groupes sociaux marginalisés ou ignorés, avec leurs problèmes, leurs histoires et surtout leur grande misère. Lors de la grève des ouvriers du textile de Kafr Al-Dawar, en février 2007, le journaliste détaille ainsi le salaire mensuel d'un ouvrier de la société après vingt-cinq ans d'ancienneté: «Avec 240 livres par mois [à peu près 30 euros], I'ouvrier peut survivre avec sa femme et un seul enfant à charge, mais à condition de ne dépenser aucun sou sur le logement, les transports, l'électricité, le gaz, les habits et la scolarisation du gosse, tout en essayant d'éviter de tomber malade !» (Al-Dustûr, 14 février 2007). Lors du sit-in des employés de l'impôt foncier devant le siège du conseil des ministres en décembre 2007, une des mobilisations emblématiques des années 2000 et qui durera onze jours, le journaliste Mostapha Bassiouny, qui campe avec les manifestants, raconte le «calvaire» quotidien d'une dizaine de milliers de fonctionnaires venus de tous les gouvernorats d'Égypte pour réclamer leur rattachement au ministère des Finances, afin de bénéficier de salaires et de primes similaires à ceux de leurs collègues travaillant aux impôts généraux. Les témoignages et récits rapportés par le journaliste sur la vie quotidienne au sit-in, sur les raisons qui ont forcé des milliers de fonctionnaires à venir protester au Caire, informent sur ce rôle nouveau qu'a joué la presse privée dans le dévoilement des conditions de vie de catégories sociales fortement marginalisées.

«Concernant les protestations sociales, le niveau de participation était très grand, celui des femmes aussi ; après ça les choses ont évolué, on parle de grèves qui se produisent au-delà des murs des usines, les gens ont commencé à investir la rue, la grève des fonctionnaires de l'impôt foncier se passait devant le siège du conseil des ministres; après ça, il y avait les employés des "centres d'informations", ils sont restés un mois devant le Parlement! Donc les gens ont commencé à protester devant les lieux du pouvoir, et, le plus important, on commence à se demander pourquoi ces gens font la grève. Donc les revendications montent à la surface et les conditions de vie des gens apparaissent au grand jour, là on parle de gens qui touchent 100 livres par mois (à peu près 15 euros), concernant les employés de l'impôt foncier! Même le Premier ministre de l'époque n'arrivait pas à croire qu'il y a des gens qui travaillent avec un tel salaire! Ce qui vous montre à quel point la réalité des conditions de vie des gens 
était étouffée dans ce pays.» (Entretien avec Kamal 'Abbas, Le Caire, 2014, traduit par l'auteur)

\section{Khaled El-Balchy ou le nouveau Sisyphe de la presse égyptienne}

Figure emblématique de la presse égyptienne, Khaled El-Balchy a joué un rôle central dans la définition des caractéristiques de la presse privée et la mutation des fonctions traditionnellement allouées aux médias en Égypte, centrés historiquement sur les décideurs politiques, vers un journalisme qui porte un regard nouveau sur le «mouvement social» et ce qu'il appelle «les gens ordinaires». Son parcours professionnel, marqué notamment par ses engagements pour l'accomplissement de la justice sociale et la défense des droits de l'homme, illustre parfaitement le combat d'une partie des fondateurs de la presse privée pour la réalisation d'un nouveau journalisme alliant indépendance éditoriale, stabilité professionnelle et engagement journalistique pour les catégories sociales défavorisées ou opprimées. Le suivi des principales étapes de sa carrière, partagées entre une multitude de structures journalistiques, atteste également des difficultés d'une presse critique à l'égard du régime et très ancré à gauche pour s'imposer dans le cadre de journaux financés par des hommes d'affaires.

Diplômé de journalisme en 1994 à l'université du Caire, Khaled El-Balchy démarre sa carrière dans la presse écrite à travers une des publications du parti socialiste égyptien, Le Rassemblement, la revue La Gauche, dont il devient en moins d'un an le secrétaire de rédaction (1996-2001). Parallèlement, il dirige et participe à la fondation de plusieurs publications émanant d'organisations de la société civile concernées par les droits des femmes et la question ouvrière en Égypte, telle la revue Kalam Sanay'iyyat (paroles d'artisans). Après un passage mouvementé dans la revue étatique Rose al-Youssef (1997-2003), il est appelé par Ibrahim Eissa pour participer à la refondation de I'hebdomadaire Al-Dustûr. Nommé rédacteur en chef adjoint, il est alors responsable des questions en lien avec les «libertés et la justice sociale de façon générale, la torture et la question ouvrière en particulier ${ }^{20} »$. C'est à son instigation que le journal se démarque par une attention particulière pour la "question sociale», les manifestations du mouvement Kifayat, dont il fut membre, les mobilisations des magistrats pour l'indépendance de la justice, mais surtout les pratiques de torture dans

\footnotetext{
${ }^{20}$ Entretien avec Khaled El-Balchy, 2014, au siège du journal en ligne Al-Bidayat (Le Début), Le Caire.
} 
les commissariats de police et dont il signe lui-même nombre d'enquêtes sur le sujet.

«Pour les premiers numéros du journal Al-Dustûr, j'avais préparé un très grand nombre de sujets, la plupart sur les pratiques de torture dans les prisons et les postes de police. On était les premiers à traiter de la question des détenus de Guantanamo transférés en Égypte pour être torturés. Mais, après le quatrième numéro, le propriétaire du journal me demande de baisser un peu le ton sur le sujet. Une statistique montrait en effet qu'en trois numéros, Khaled Al-Balchy et son équipe avaient publié une quarantaine d'articles sur la question de la torture, dont treize rédigés par lui-même.» (Entretien avec Khaled El-Balchy, 2014, au siège du journal en ligne Al-Bidayat (le début), Le Caire, traduit par l'auteur)

Malgré la célébrité et le succès commercial du journal, qui enregistre dès 2005 le tirage le plus élevé parmi les hebdomadaires égyptiens, les conditions de production sont difficiles, et les salaires très bas des jeunes rédacteurs génèrent des conflits permanents avec le rédacteur en chef, Ibrahim Eissa, et le financeur du journal, l'éditeur 'Issam Ismaïl Fahmy. D'après Khaled Al-Balchy, le salaire le plus élevé d'un jeune rédacteur en 2006, "pour un bon journaliste», était situé aux environs de 800 livres égyptiennes (à peu près 80 euros à l'époque). Mais la plupart des journalistes, lorsqu'ils étaient payés, percevaient un salaire mensuel qui variait entre 20 et 40 euros. Ces difficultés posent dès le départ les limites de l'équation économique établie par l'éditeur 'Issam Ismaïl Fahmy pour répondre aux missions justicières dont le journal se voulait le héraut et les contraintes politiques et budgétaires dans lesquelles opérait l'entreprise de presse sous Moubarak. L'équation est parfaitement synthétisée par Ibrahim Eissa: "La liberté en contrepartie d'un gain financier réduit» ('Abiyyat et Habib, 2010, p. 15). Concrètement, il s'agissait pour l'éditeur, afin de faire face à la censure publicitaire exercée par le régime, de pouvoir générer du bénéfice uniquement grâce à la distribution, de réduire au maximum les coûts de production et en fixant le seuil de la diffusion à 130000 exemplaires. Pour compenser les limites du budget, le journal repose principalement, en premier lieu, sur sa réputation et la notoriété intellectuelle et professionnelle de ses journalistes fondateurs, notamment Ibrahim Eissa. Ce dernier permet d'assurer la collaboration occasionnelle ou régulière d'écrivains de renom, de personnalités médiatiques, universitaires et politiques. En second lieu, la direction du journal recourt au prolongement indéfini des périodes de stage et compte sur l'engagement politique des rédacteurs. La plupart des anciens rédacteurs du journal avec qui nous avons parlé considèrent en effet «l'expérience Al-Dustûr» comme relevant d'une «mission nationale» 
contre «le despotisme», «l'injustice » et «pour la liberté», justifiant alors les sacrifices fournis en termes financiers et la dureté des conditions de travail.

«La question des salaires posait évidemment problème, parce qu'on travaillait beaucoup et que les salaires étaient très bas, mais personnellement j'étais satisfaite. Aujourd'hui, franchement, je regarde le salaire avant de regarder le travail, mais à l'époque du journal Al-Dustûr, je pensais d'abord au travail. Al-Dustûr était une expérience complètement différente et qui m'apportait une satisfaction indescriptible. Je peux gagner le triple aujourd'hui avec le quotidien Al-Watan (La Nation) mais ne fournir que le quart du travail que je faisais pour Al-Dustûr.» (Entretien avec une ancienne journaliste d'Al-Dustûr, Le Caire, 2014, traduit par l'auteur)

Les chiffres de diffusion pour les mois de janvier et février 2007 (Al-Dustûr, le 28 février 2007) attestent de la réussite relative de cette formule économique. Elle permettait au journal de survivre financièrement et de défendre son projet éditorial, mais avec des «dépenses réduites et des marges bénéficiaires réduites» ('Abiyyat et Habib, 2010, p. 15). Le document officiel fourni par la direction générale de la distribution de l'institution Al-Ahrâm, publié par le journal Al-Dustûr lors de la mise en examen de l'éditeur 'Issam Ismaïl Fahmy pour fraude fiscale, montre en effet un niveau de diffusion assez stable, s'établissant autour de 130000 exemplaires, et une moyenne d'exemplaires non vendus située entre 5 et $8 \%$ (Al-Dustûr, 28 février 2007). Selon les chiffres fournis par Ibrahim Eissa, le journal hebdomadaire, vendu 2 livres, rapportait 50000 livres de bénéfices (écart entre impression et distribution) chaque mercredi, le jour de livraison du Dustûr ('Abiyyat, Habib, 2010, p. 16). Les difficultés et les tensions internes au sein du journal s'accentuent quand il cherche à reproduire la même formule économique pour la parution quotidienne. Cette dernière s'appuie alors sur un budget mensuel très inférieur à celui des journaux concurrents - 165000 livres contre 1,2 million de livres pour le quotidien Al-Masry Al-Youm -, un nombre très élevé de rédacteurs et des conditions matérielles de travail encore plus difficiles. Outre les revenus très faibles et irréguliers, les journalistes du Dustûr décrivent une salle de rédaction composée de deux pièces et pauvrement meublée, dans laquelle il y a un seul ordinateur pour toute l'équipe de rédaction et «rarement utilisable à cause des virus» et un téléphone fixe qui coupe toutes les trois minutes par souci d'économie. Aucune voiture de transport n'est mise à la disposition des journalistes ${ }^{21}$. Avec 85000 exemplaires imprimés et très peu de retours, le quotidien

${ }^{21}$ Entretien avec le journaliste Amrou Badr, dans un café dans le centre du Caire, 2013. 
Al-Dustûr, vendu une livre dans les points de vente, arrive toutefois à dégager un faible bénéfice de 6000 livres par jour grâce à la distribution, mais il s'expose aux protestations salariales permanentes des rédacteurs, dont le nombre a doublé avec la parution quotidienne. Le journal est aussi incapable de résister durablement à la puissance financière et aux assauts des quotidiens Al-Masry Al-Youm et Al-Shoroûk ${ }^{22}$, dont les lignes éditoriales sont certes moins engagées mais qui offrent un cadre de travail plus sécurisant avec de meilleures rémunérations. Trois ans après sa fondation, le journal Al-Dustûr avait déjà perdu nombre de ses chroniqueurs, rédacteurs et caricaturistes célèbres, dont le caricaturiste Amrou Salim, le journaliste Mostapha Al-Bassiouny et l'écrivain Bilal Fadl. Khaled El-Balchy, qui se chargeait de négocier avec la direction l'augmentation des salaires, avait quitté le journal dès novembre 2006 pour protester contre les «prélèvements répétitifs et injustes des salaires des rédacteurs ${ }^{23}$ ».

Après sa démission, Khaled El-Balchy est «quasiment à la rue ${ }^{24}$ ». II travaille au secrétariat de rédaction de plusieurs journaux égyptiens, où il est chargé de réécrire les articles "pour gagner de quoi vivre». II lance parallèlement un nouveau journal, Al-Nahar (Le Jour), avec une licence de publication étrangère (à Chypre), mais sans parvenir à le faire publier puisqu'il est censuré par la police politique du régime, Amn al-dawla (sécurité de l'État). Au début de l'année 2007, il fait partie de l'équipe chargée de fonder le quotidien privé Al-Badîl (L'alternative), sous la direction d'un célèbre journaliste et intellectuel de la gauche égyptienne, Ahmed El-Sayyed Saïd. L'expérience de ce titre (Ghiglia, 2015) représente la seconde étape marquante dans la carrière du journaliste Khaled El-Balchy après celle du Dustûr. Fondé par des journalistes et des activistes en rupture avec les structures traditionnelles de la gauche égyptienne, financé par des hommes d'affaires "désintéressés financièrement", Al-Badîl a pour mission première de proposer une alternative "civile» au "projet islamiste» et à l'autoritarisme du régime. Le journal se focalise dès le départ sur la couverture des mouvements sociaux, les affaires de droits de l'homme, la publication des rapports des centres de recherche et ouvre grandement ses pages aux forces politiques de l'opposition. Chargé au départ de former le "service des enquêtes», l'autorité de Khaled El-Balchy s'étendra rapidement

\footnotetext{
${ }^{22}$ Al-Shoroûk (l'aube) est un quotidien fondé en 2009 par I'homme d'affaires égyptien et PDG de la maison d'édition Al-Shoroûk, Ibrahim El-Mo'allem.

${ }^{23}$ Entretien avec Khaled El-Balchy au siège du journal en ligne Al-Bidayât, Le Caire, 2014. 24 lbid.
} 
au «services des informations» puis, "pour des raisons et des difficultés multiples», il devient responsable de la plupart des rubriques du journal. II engage alors ce qu'on pourrait considérer comme une restructuration du quotidien, en procédant notamment à une réévaluation des rédacteurs et à des transformations de forme afin d'améliorer l'attractivité du quotidien qui peine sur le plan des ventes. Au cours de l'année 2008, Khaled El-Balchy est logiquement promu directeur de rédaction, puis rédacteur en chef en octobre de la même année, après l'aggravation de l'état de santé d'Ahmed El-Sayeed Said. En dépit de ces changements et la promesse des financeurs d'augmenter le budget du journal, Al-Badîl, qui ne bénéficie par ailleurs d'aucune rentrée publicitaire à cause de la pression exercée par le régime de Moubarak sur les annonceurs, cesse définitivement de paraître en avril 2009.

«Al-Badîl a fermé en avril en 2009. Les financeurs du journal ont déclaré que c'était pour des raisons financières. Moi je pense que ce n'est pas la vraie raison. Je pense que c'est plutôt pour des raisons politiques. Le journal Al-Badîl est un journal de gauche, il est né pour couvrir et soutenir les mouvements sociaux, notamment le mouvement ouvrier. II devait être l'expression profonde et sincère de ce mouvement social que connaissait la société égyptienne durant les années 2000. Et les principaux financeurs du journal ont investi dans le journal al-Badil par conviction pour ce projet. Mais je pense qu'ils n'ont pas mesuré ou ont sous-estimé le prix à payer pour exprimer ce mouvement protestataire de la société, surtout qu'Al-Badîl était le journal le plus radical dans la critique du régime.» (Entretien avec Khaled El-Balchy au siège du journal en ligne Al-Bidayât, Le Caire, 2014, traduit par l'auteur)

Après l'arrêt du journal, Khaled El-Balchy est «encore une fois à la rue ». II entreprend alors une série d'initiatives afin de faire revivre l'expérience d'Al-Badîl, sans jamais parvenir à convaincre les anciens financeurs de reprendre le journal, ni à fonder ou s'établir durablement dans des structures similaires à ce dernier. Son parcours professionnel entre 2009 et 2020 correspond, in fine, à celui de la plupart des journalistes de la presse privée sur lesquels porte ce chapitre, traversé par des allers-retours forcés entre presse papier, presse en ligne et réseaux sociaux, Facebook notamment. La presse en ligne et les réseaux sociaux constituent dès la fin des années 2000 à la fois des espaces de règlement de compte et de critique interne des médias, et des alternatives pour des journalistes égyptiens de produire un journalisme souvent engagé et rarement couronné de succès, échappant à la censure politique et aux contraintes imposées à l'industrie de la presse papier.

«J'ai passé un an et demi à essayer de convaincre les propriétaires de reprendre l'expérience d'Al-Badîl. En 2010, on s'était mis d'accord sur la 
création d'un hebdomadaire en édition papier, en plus d'un quotidien en ligne. Effectivement, on avait fait quelques pas dans la réalisation du projet, mais à la fin ils ont renoncé. J'ai donc décidé de lancer un journal en ligne avec mes propres fonds, appelé Al-Badîl Al-Jadid (le nouveau Al-Badîl). Après la révolution, les propriétaires sont revenus me voir pour reprendre l'expérience du quotidien, mais encore une fois ils ont fait marche arrière, puis ils ont vendu la société Al-Badîl tout entière à un groupe de personnes que je croyais capable de sauver le journal, d'autant plus qu'à cette époque je n'avais plus d'argent, et ils avaient promis de ne pas toucher à la ligne éditoriale. Mais à la fin, ils ont changé la ligne éditoriale du journal, et j'ai donc décidé de partir. J'ai fondé après ça un journal en ligne, avec très peu de moyens, appelé Al-Bidayat (Le Début), mais je n'ai pas réussi à le garder longtemps. Après le 30 juin 2013, on m'a proposé d'être le rédacteur en chef du journal privé appelé $A I$-Wadi (L'Oued). Effectivement j'ai travaillé pendant six mois sur le projet et on a lancé deux numéros, mais au troisième on m'a annoncé l'arrêt du journal pour des raisons financières (rires) ! Mais ça c'est au troisième numéro, donc ils ne peuvent parler de crise financière cette fois-ci ! Mais le journal Al-Wadi continue de paraître après mon départ, comme c'est le cas pour Al-Badîl. Aujourd'hui j'essaye de reprendre l'expérience du site Al-Bidayat.» (Entretien avec Khaled El-Balchy au siège du journal en ligne Al-Bidayât, Le Caire, 2014, traduit par l'auteur)

\section{Conclusion}

À travers l'étude des biographies professionnelles de journalistes du «mouvement social», cette contribution avait pour ambition de revenir à la fois sur une nouvelle figure du «journalisme-justicier» en Égypte, mais également sur les limites des modèles économiques et professionnels portés par le secteur privé. Entre reconfigurations politiques, logiques économiques et répression policière, le secteur privé, sous toutes ses formes, a été incapable de proposer une alternative durable aux organisations médiatiques officielles ou officieuses. Le développement des sites d'information en ligne et autofinancés, tel Al-Bidayat, marque ainsi la fin en Égypte du journal papier financé par des "hommes d'affaires» comme alternative au modèle du journal étatique au service du «prince». L'extension du grand capital au secteur des médias avait constitué durant les années 2000 la solution historique face aux différentes contraintes imposées depuis les années 1960 pour empêcher l'émergence d'une presse critique à l'égard du pouvoir. Son incapacité à faire face à la répression du régime d'Abdel Fatah El-Sissi, à défendre «les idéaux du 25 janvier 2011 » et l'implication de ses figures fondatrices dans le retour de l'armée au pouvoir conduisent à situer désormais la dynamique de contestation politique et 
sociale en Égypte en dehors de l'industrie de la presse papier. Depuis le coup d'État militaire de juillet 2013, les rapports et témoignages qui parviennent chaque jour à travers les organisations de droits de l'homme sur l'agression, l'emprisonnement ou la disparition de journalistes renseignent ainsi sur une situation actuelle de «terreur». Au nom de la «restauration de l'autorité de l'État» et afin de reprendre le contrôle des médias, le régime actuel a mis progressivement en place tout un dispositif répressif qui contribue à reconfigurer en profondeur les figures du «journalisme-justicier» en Égypte, centré désormais non plus sur le mouvement social mais sur les questions des droits de l'homme et le suivi des procès intentés à l'encontre des professionnels des médias, des opposants politiques, des activistes ou des acteurs de la société civile.

Tout d'abord, le changement de législation, avec la promulgation en 2018 de la loi n 180 sur «l'organisation de la presse, des médias audiovisuels et du Conseil supérieur pour l'organisation des médias», a des effets notoires. Non seulement ce texte, qui remplace celui de 1996 et ses divers amendements, durcit la réglementation en vigueur, mais il étend pour la première fois la possibilité de censure aux réseaux sociaux, pour les comptes dont le nombre de suiveurs est égal ou supérieur à 5000 (article 19), et exige pour les «sites numériques ${ }^{25}$ » l'obtention d'une licence de diffusion.

Ensuite, les instances de "régulation» censées "garantir la liberté de la presse » qui ont été créées sont dirigées par d'anciennes figures du régime de Moubarak. C'est le cas notamment du Conseil supérieur des médias (loi $n^{\circ} 180$ de 2018) dont le président est nommé par le président de la République (article 73). Dirigée jusqu'à récemment par l'ancien doyen du syndicat des journalistes, Makram Mohammed Ahmed, cette instance peut, en plus de la responsabilité pénale des personnes en cause, bloquer (hajb) un site d'information, un blog ou un compte pour des motifs aussi vagues que «la publication de fausses informations» ou «l'incitation à la violence et à la haine» (article 19). Depuis mai 2017, plus de 500 «sites électroniques» ont été bloqués (Freedom of Thought and Expression, 2019), sans jamais connaître officiellement ni les raisons ni l'origine de la censure, car la nouvelle «loi contre la cybercriminalité » (2018) permet également à la police et au procureur général de bloquer des sites en ligne dont les contenus «constituent une menace pour la sécurité nationale» ou

\footnotetext{
${ }^{25}$ Le terme «site numérique» est très ambigu. II est défini dans la loi par «la page, le lien ou l'application électronique autorisée et qui fournit un contenu journalistique, médiatique ou publicitaire quel qu'il soit» (article 1).
} 
«l'économie du pays» (article 7). Tous les journaux en ligne fondés par le journaliste Khaled El-Balchy ont ainsi été censurés par le régime: Al-Bidayat, bloqué le 11 juin 2017; Kateb (Auteur), bloqué quelques heures seulement après son lancement, le 25 août 2018 et, plus récemment, le journal en ligne Darb (Voie), le 9 avril 2020, un mois seulement après son lancement.

Enfin, un processus de confiscation politique de groupes médiatiques privés s'est développé à travers une société appartenant aux services de renseignement, Eagl Capital, qui était inconnue jusqu'alors des milieux d'affaires égyptiens (Bahgat, 2017) ${ }^{26}$. Contrairement ainsi aux années Moubarak, le régime actuel ne se satisfait plus d'exercer un contrôle indirect sur les médias à travers des pressions économiques sur les propriétaires.

\section{Bibliographie}

'ABIYYAT Hicham, Habib Nancy, Al-Dustûr, histoire d'un pays et d'un journal, Le Caire, Dar Al-Masry, 2010 (en arabe).

Abdalla Iskandar Nadine, Les Mobilisations sociales en Égypte entre revendications économiques et opposition au régime (2005-2009): étude de cas de deux mouvements sociaux: le mouvement des ouvriers de la Compagnie de Méhalla (2006-2008) et celui des fonctionnaires de l'impôt foncier (2007-2009), Grenoble, thèse de doctorat en science politique, École doctorale des sciences de l'homme, du politique et du territoire, 2014. https://tel.archives-ouvertes.fr/tel-01286568/

Al-AzBaou Youssri, La Révolution du 25 janvier 2011, vers une nouvelle organisation syndicale, Le Caire, Centre des études politiques et stratégiques d'Al-Ahrâm, 2011 (en arabe).

BENAZIZ Bachir, "Journaliste-conseiller du prince" et "journaliste-justicier": figures du journalisme et transformation de la presse écrite en Égypte», dans La Culture et ses dépendances: les productions culturelles et leurs circulations au Maghreb et au Moyen-Orient, sous la dir. d'A. Benchenna et D. Marchetti, Beyrouth-Rabat, Presses de I'IFPO et éditions du CJB, coll. Co-éditions, chapitre 1, 2021.

BENAZIZ Bachir, "Al-Dustûr/Al-Tahrîr, apogée et déclin d'un journal privé», Revue Tiers Monde, $\mathrm{n}^{\circ}$ 222, 2015. DOI: https://doi.org/10.3917/rtm.222.0031

\footnotetext{
${ }^{26}$ On se reportera également utilement sur ce point au chapitre 5 de ce livre rédigé par Maria Adib Doss.
} 
DUBOC Marie, Contester sans organisations: stratégies de mobilisation, question sociale et espace de visibilité dans les grèves de l'industrie textile égyptienne, 2004-2010, thèse de doctorat en sociologie, Paris, École des hautes études en sciences sociales, 2012.

EL-KHAWAGA Dina, "Sisyphe ou les avatars du nouveau journalisme égyptien », Égypte/Monde arabe, $n^{\circ}$ 3, 2000. DOI: https://doi.org/10.4000/ema.803

FREEDOM OF THOUGHT AND EXPRESSION, 2019. https://afteegypt.org/en/blockedwebsites-list

GHIGLIA Mariana, "Al-Badîl, ou l'Alternative: récit d'une expérience à la croisée entre journalisme et engagement militant», Égypte/Monde arabe, n 12, 2015. DOI: https://doi.org/10.4000/ema.3428

Hamamou Sabah, Mémoires d'une journaliste d'Al-Ahrâm: vingt ans dans la vieille institution journalistique, Le Caire, autoédité, 2012 (en arabe).

LEMIEUX Cyril, «Les formats de l'égalitarisme: transformations et limites de la figure du journalisme-justicier dans la France contemporaine», Quaderni, n 45, 2001. DOI: https://doi.org/10.3406/quad.2001.1497

LEVÊQUE Sandrine, Les Journalistes sociaux: histoire et sociologie d'une spécialité journalistique, Rennes, Presses universitaires de Rennes, 2020 [2004]. DOI: https://doi.org/10.4000/books.pur.24697

SCHLESINGER Philip, "Repenser la sociologie du journalisme: les stratégies de la source d'information et les limites du média-centrisme», Réseaux, n 51, 1992. DOI: https://doi.org/10.3406/reso.1992.1926

STEUER Clément, "Le printemps des partis ? Le rôle des organisations partisanes égyptiennes dans les élections législatives», Confluences Méditerranée, $\mathrm{n}^{\circ}$ 82, 2012. DOI :https://doi.org/10.3917/come.082.0091

\section{Sources}

AL-DUSTÛR, "Où est parti l'argent du secteur public», n 51, 8 mars 2006.

AL-DUSTÛR, "Kafr Al-Dawar, la forteresse de l'industrie qui plonge dans le chômage ", n 65, 14 juin 2006.

AL-DUSTÛR, "Al-Mahalla Al-Kûbra qu'on a rapetissé », $\mathrm{n}^{\circ}$ 71, 26 juillet 2006.

AL-DUSTÛR, n 100, 14 février 2007.

AL-DUSTÛR, no 102, 28 février 2007.

BAHGAT Hossam, "Enquête sur l'acquisition de la société "Médias des Égyptiens" par les services de renseignement", Madamasr, 20 décembre 2017. https://www madamasr.com/ar/2017/12/20/feature / 



\title{
Chapitre 5 \\ L'audiovisuel en Égypte de 2000 à 2020: un secteur sous contrôle
}

\author{
Maria Adib Doss
}

L'histoire de l'audiovisuel en Égypte est étroitement liée à l'histoire politique de ce pays. Elle dépend surtout de la conception que les différents régimes ont eu du rôle des médias dans la vie politique et sociale. Ce chapitre voudrait ainsi restituer l'évolution des modalités de contrôle du secteur audiovisuel et, plus précisément, celui en lien avec l'information. Ce rapport se manifeste par ce que les professionnels du métier en Égypte appellent «la logique de gestion» par le pouvoir de ce secteur jugé stratégique. Toute la subtilité de ce terme réside dans le fait que le fonctionnement du champ médiatique est en grande partie tributaire de la conception que se fait le pouvoir politique du rôle et de la place que doivent occuper les médias dans la société. C'est de cette conception que dépend la mise en œuvre d'outils de contrôle.

Cette contribution analyse deux configurations différentes. L'une est relative à un "autoritarisme souple" sous la dernière décennie des mandats (1981-2011) de Hosni Moubarak, notamment avec la montée d'une opposition puissante. L'autre porte sur l'Égypte post-2013. La première configuration est marquée par la démonopolisation du secteur audiovisuel dans les années 1990-2000, Hosni Moubarak adaptant ses modes de contrôle. L'éditorialiste et universitaire Mamoun Fandy décrit cette période comme le règne «de l'ambiguïté» (Fandy, 2007, p. 23-24), avec une multitude d'instances régulatrices dont les prérogatives se chevauchaient parfois. Les relations entre les gouvernements successifs et les médias sont en effet très complexes et ne suivent pas des logiques linéaires, la censure étant élastique et activée ou non selon des règles indéfinissables et de manière arbitraire. La chercheure Tourya Guaaybess appelle cette forme de contrôle 
«la politique du Stop and go» (Guaaybess, 2013, p. 49). Ces règles dépendent entre autres et en grande partie de ce que le chercheur Adel Iskandar appelle «les politiques souterraines» (Iskandar, 2014, p. 252) ou les relations informelles entre les services de sécurité et les professionnels des médias.

Après le départ forcé de Mohamed Morsi ${ }^{1}$ en juillet 2013 s'ouvre une nouvelle configuration fondamentalement différente de celle sous Moubarak. Les véritables intentions du nouveau pouvoir politique en Égypte à l'égard des médias n'ont dès lors pas cessé de s'affirmer, le candidat et président Al-Sissi s'exprimant souvent sur la question. Sa première déclaration publique à ce sujet remonte à 2014, lors du lancement des travaux d'élargissement du canal de Suez, où il enviait Gamal Abdel Nasser pour son appareil médiatique et sa faculté à mobiliser la population derrière lui (Abbas, 2014). La promulgation, la même année, de lois instaurant trois instances de régulation des médias constitue au fond la concrétisation de cette nouvelle conception du rôle des médias dans la société. Autrement dit, les modalités de contrôle des contenus médiatiques sont inséparables à la fois des textes qui les régissent, de la «logique de gestion» du secteur des médias et de la conception que se fait le régime de ce dernier. La multiplication des niveaux de contrôle, le règne de la peur et des «lignes rouges» insaisissables, mais intériorisées par les journalistes, restent des caractéristiques inchangeables. La différence réside dans la nature et l'étendue du champ des possibles des discours publics tolérés par rapport aux questions socio-politiques et des messages que le régime en place cherche à faire passer directement ou indirectement. Les professionnels des médias en Égypte n'ignorent bien évidemment pas ce mode de fonctionnement, conscients des contraintes politiques et des formes de contrôle qui s'imposent à eux.

Dans une approche comparative, ce chapitre examine donc successivement les trois modalités de contrôle de l'audiovisuel sous Hosni Moubarak et Abdel Fattah Al-Sissi : la régulation formelle et institutionnelle, notamment les lois qui régissent ce secteur en Égypte, les modes de propriété et enfin les modalités de la régulation informelle. Ce travail est le fruit d'une enquête de terrain par entretien auprès des professionnels des médias en Égypte et d'une analyse des textes juridiques et de documents officiels. Une partie des entretiens a été effectuée dans le cadre de ma thèse, entre 2013-2018, complété par une autre enquête en 2020.

\footnotetext{
${ }^{1}$ Mohamed Morsi, candidat des Frères musulmans, fut président de la République entre juin 2012 et juillet 2013.
} 


\section{La régulation formelle et institutionnelle}

\section{Le règne de l'ambiguiité sous Moubarak}

En 2001, la création de Dream TV, la première chaîne satellitaire privée, fondée par l'homme d'affaires égyptien Ahmed Bahgat, a constitué une rupture avec le monopole de l'État sur l'information. Un système de régulation dual du secteur audiovisuel s'est ainsi établi. Certaines règles concernent le secteur audiovisuel public, notamment l'Union de la radio et de la télévision égyptienne (URTE), régie par la loi 13/1979, rectifiée par la loi 223/1989, et dépendent donc directement du ministère de I'Information ${ }^{2}$. Tout le personnel de la télévision et de la radio publique est composé d'employés de l'État égyptien, c'est-à-dire astreints à ne pas dépasser les «lignes rouges», sur lesquelles on reviendra plus loin, sous peine d'être immédiatement licenciés.

En ce qui concerne les chaînes satellitaires privées, il n'existait jusqu'en 2016 aucune règle ni décret dédiés à ce secteur. Tout laisse à croire que l'absence de textes relevait d'une volonté politique afin de garder le contrôle sur les propriétaires. Formellement, ces nouvelles chaînes de télévision dépendaient des réglementations des zones franches, qui relevaient elles-mêmes de la compétence de l'Autorité générale des investissements, c'est-à-dire in fine du ministère de l'Investissement. Travailler au sein de ces zones nécessitait donc l'obtention d'une licence de cet organisme, qui conservait également un droit de révocation (Guaaybess, 2013, p. 60). Si les chaînes satellitaires opérant dans la zone franche appelée Cité des médias ${ }^{3}$ ne sont pas soumises aux lois de censure qui régissent les médias publics, elles sont tenues de respecter les règles de la Cité. Située à proximité du Caire, dans la nouvelle ville du " 6 Octobre», celle-ci regroupe un grand nombre de studios de tournage, un hôtel cinq étoiles, des amphithéâtres et un parc d'attractions comparable à Disneyland nommé Magic Land. La Cité des médias créée en 2002 est gérée par une compagnie, l'Egyptian Media Production Company (EMPC), dont I'Union de la radio et de la télévision égyptienne (URTE) est aussi le principal actionnaire, ce qui lui assure le pouvoir décisionnel. Les contenus de leurs programmes sont ainsi soumis à l'Autorité générale des investissements. Pour obtenir une licence de

\footnotetext{
${ }^{2}$ La dernière Constitution de 2014 a remplacé le ministère de l'Information par une autre instance responsable de la régulation de l'audiovisuel dans son ensemble: le Conseil suprême de régulation des médias.

${ }^{3}$ La Cité des médias est un complexe dédié à la production médiatique et cinématographique.
} 
diffusion, il faut passer par plusieurs niveaux (voir le schéma 1). L'autorisation de l'instance mentionnée constitue une première étape de filtrage. Après la présentation de la demande, l'Autorité générale des investissements vérifie certaines règles formelles, concernant le nom, par exemple, ou les avis d'imposition de la société et son capital, et surtout s'appuie sur les investigations sécuritaires menées par Amn al-dawla (le service de sécurité de l'État) ${ }^{4}$. Une fois cette étape franchie, l'entreprise se dirige vers La Cité des médias pour louer les studios et les matériels. Enfin, elle doit passer par la compagnie Egyptian Satellite Channel (ESC), qui gère les satellites Nile Sat et la location de ses canaux de diffusion. Le principal actionnaire de la compagnie ESC et de la Cité des médias reste I'URTE, avec respectivement des parts de $42 \%$ et $43,2 \%{ }^{5}$, ce qui permet à l'État d'en garder la mainmise.

\section{Schéma 1}

\section{Organisation de la zone franche}

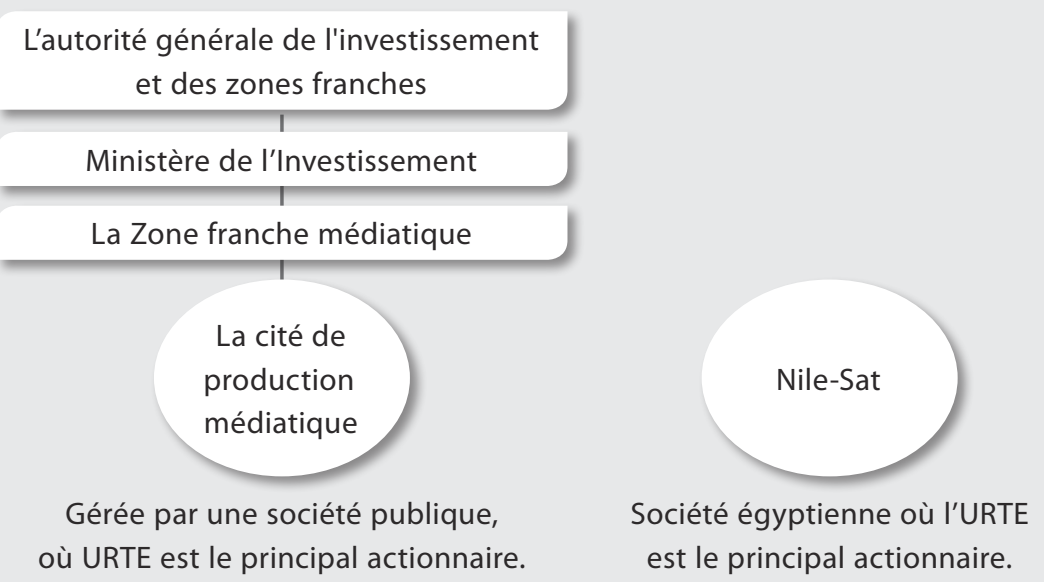

\footnotetext{
${ }^{4}$ Entretien réalisé en 2013 avec des fonctionnaires de la zone franche des médias.

${ }^{5}$ Le chiffre qui a été avancé par plusieurs interviewés en 2013 et 2014, notamment les fonctionnaires de la zone franche dédiée aux médias, était estimé à $50 \%$. Selon le dernier rapport officiel daté du 11 juillet 2017 et intitulé «Formulaire de divulgation du conseil de l'administration et la structure des actionnaires pour la période terminée le 30/06/2017 de la société égyptienne de la cité des médias ", la part de l'URTE (désormais Autorité nationale des médias) est de $43 \%$. Ce rapport est disponible en arabe (sur le site de la bourse égyptienne: http://egx.com.eg/English/newsdetails.aspx?NewsID=163165. En 2017, le directeur général de la société égyptienne de la Cité des médias était Ossama Haikal, l'ex-ministre de l'Information sous le Conseil suprême des forces armées.
} 
Selon l'ultime forme du contrat, seules les chaînes généralistes et non les chaînes d'information en continu étaient autorisées à diffuser à partir de la Cité des médias. Cela s'étendait également aux contenus, dont les journaux télévisés et le fil d'information en continu en bas de l'écran. C'est ce qui explique, par exemple, que les talk-shows socio-politiques, qui ont marqué les années 2000, aient été définis comme étant des «émissions sociales» et, dans une moindre mesure, des "émissions sociales et d'affaires courantes».

En signant ce contrat avec l'Autorité de l'investissement, les chaînes approuvaient également les règles et principes de travail de la zone franche des médias, ainsi que la charte éthique du travail médiatique la Ligue arabe de juin 2002. Les articles de la charte de régulation de la Cité des médias sont caractérisés par une formulation générale et élastique, sujette à des interprétations diverses. Héritière des grandes formules qui ont constitué le méta-discours national, la première clause prohibe par exemple de "porter atteinte à l'intérêt national de l'État», sans le définir. La deuxième insiste sur le respect des directives du gouvernement en cas «de crise», de "catastrophes naturelles et de guerres». La charte prohibe enfin la diffusion de contenus incitant à la haine, à la diffamation des religions, aux conflits confessionnels, ainsi qu'à la diffusion de toute matière télévisuelle qui ne convienne pas aux principes et éthiques de la société ${ }^{6}$.

\section{Le Conseil suprême des médias sous AI-Sissi : la parole confisquée}

Trois instances ont été mises en place conformément à la loi de 2016 $\left(n^{\circ} 92\right)$ et par la suite à celle de 2018 ( $\left.n^{\circ} 180\right)$ appelée «loi de la régulation de la presse et des médias et du Conseil suprême de régulation des médias ». Ces trois instances, censées remplacer à la fois le Conseil suprême de la presse et l'URTE' ${ }^{7}$, sont les suivantes: le Conseil suprême de la régulation des médias, l'Autorité nationale de la presse et l'Autorité nationale des médias audiovisuels. Nous nous concentrons ici sur le seul Conseil suprême de la régulation des médias, les deux dernières instances ayant pour mission de gérer les journaux officiels et les chaînes du service public. Pour comprendre

\footnotetext{
${ }^{6}$ Document officiel des «Règles et principes généraux de travail et de diffusion à partir de la zone franche médiatique», conformément à la décision du conseil d'administration de la zone franche médiatique, $n^{\circ}$ 6/42-2011, lors de sa réunion du 21 avril 2011.

${ }^{7}$ L'URTE est la compagnie nationale de radio-télévision égyptienne. Elle a été fondée en 1970 sous Nasser. Son siège se situe dans le bâtiment Maspero sur la corniche du Nil. Elle a été placée sous la tutelle directe du ministère égyptien de l'Information. L'Union a été remplacée en 2016 par l'Autorité nationale des médias.
} 
son fonctionnement, le parti-pris a été de se focaliser, d'une part, sur sa composition et, d'autre part, sur ses prérogatives à travers les différentes chartes qu'il a publiées.

Selon les articles 69 et 70 de la loi $n^{\circ} 180$ de 2018, le Conseil suprême de la régulation des médias est l'«autorité compétente en Égypte pour la protection de la liberté d'opinion, de la pensée et de l'expression. Elle garantit l'indépendance des médias conformément aux dispositions de la Constitution et de la loi. Le conseil est responsable de l'octroi des licences pour tout travail médiatique, de l'établissement des normes et de l'évaluation des performances médiatiques. Le conseil jouit d'une indépendance totale dans toutes ses affaires selon le texte de la loi et en vertu de sa formation de représentants des médias, d'entités scientifiques populaires, élues et spécialisées».

Malgré cette définition ambitieuse, le Conseil suprême de régulation des médias est d'abord, dans les faits, un outil de censure du régime, y compris pour les médias en ligne et les réseaux sociaux, et sa composition reflète la domination du pouvoir exécutif. En effet, selon l'article 73 de la loi $n^{\circ} 180$ de 2018, le Conseil se compose de neuf membres, dont deux sont nommés directement par le président de la République: le président du Conseil et une personnalité publique proposée par le Parlement et ne faisant pas partie des députés. Le Conseil suprême de régulation des médias comprend également le vice-président du Conseil d'État, le chef de l'Autorité pour la protection de la concurrence et la prévention contre les monopoles, un représentant de l'Autorité nationale de régulation des télécommunications et un autre du Conseil suprême des universités. Le Conseil intègre également un membre du Syndicat des journalistes et un autre du Syndicat des médias, fondé en 2019. La loi oblige par ailleurs ces organes à soumettre le double du nombre de candidats prévu à leur intention dans la loi, de sorte que la décision finale de sélection incombe au président de la République. La loi donne également à ce dernier le pouvoir de nommer directement les membres émanant des deux syndicats, au cas où ces derniers présenteraient en retard leurs candidats, et cela dans les trois mois de la période précédant la fin du mandat du Conseil suprême.

D'autre part, la loi comporte une grande confusion entre les objectifs, les termes de référence et les moyens pour les atteindre. Les formulations vagues autorisent toutes sortes d'interprétations, comme le montre l'article 69-1 qui porte sur les objectifs généraux du Conseil : «la protection du droit des citoyens à bénéficier de médias audiovisuels et d'une presse libre 
et équitable en accord avec l'identité culturelle égyptienne». Le Conseil suprême de régulation des médias devient également l'unique instance qui décide de la création des journaux, des médias audiovisuels et des «sites électroniques» (article 60). De plus, le législateur attribue au Conseil le pouvoir de «recevoir des plaintes concernant ce qui est publié dans les journaux ou diffusé par les médias, et il a le droit de prendre des mesures appropriées contre le journal ou le média en violation de la loi ou des codes d'honneur » (article 70-5), ouvrant la porte à toutes sortes de sanctions telles que des amendes, des suspensions ou un blocage du média en question. Ces mesures sont détaillées dans une charte des sanctions promulguée en mars $2019^{8}$.

Le Conseil a également le pouvoir de renvoyer un journaliste ou un responsable d'un média au syndicat compétent pour des questions disciplinaires, ce qui est considéré par les journalistes membres du syndicat comme une ingérence dans les affaires des syndicats ${ }^{9}$. L'une des compétences les plus importantes du Conseil consiste à veiller à «la protection de la concurrence et [à] empêcher le monopole dans le domaine de la presse et des médias», mais aussi à «autoriser ou non tout transfert de propriété », conformément aux articles 36, 52 et 53 de la loi nº 180 de 2018. Cependant, le Conseil ignore, depuis sa création, le monopole exercé par la société Egyptian Media Group (EMG) sur le secteur médiatique et le rachat de cette dernière par «Eagle Capital pour les investissements financiers » (Bahjat, 2017). Celle-ci serait détenue par les services secrets et présidée par Dalia Khorshid, ancienne ministre de l'Investissement.

Plusieurs exemples illustrent ces propos. Deux mois après sa formation en juin 2017, le Conseil a publié un rapport de suivi des "excès» des programmes et des séries diffusées pendant le mois de ramadan sur différentes chaînes satellitaires (Hossni, 2017). Il a annoncé le même jour sa décision d'infliger une amende de 200000 livres égyptiennes (10500 euros environ au $1^{\text {er }}$ juin 2017) à chaque chaîne satellite et 100000 livres (5 250 euros) à chaque radio si un mot «offensant» était diffusé sur leurs antennes, qu'il s'agisse d'une émission, d'une fiction ou d'une publicité (Ghaly, 2017).

\footnotetext{
${ }^{8}$ La charte des sanctions est disponible en arabe sur le site du Conseil suprême: http://scm. gov.eg/17937-2/

${ }^{9}$ Entretien, 2020.
} 
En outre, le conseil recourt fréquemment à la loi, au décret réglementaire, aux chartes de sanction et aux codes d'honneur et des mœurs pour suspendre ou arrêter certains programmes télévisés, aucun genre n'étant épargné. À titre d'exemple, le programme "SNL en arabe ${ }^{10}$ » a été (arrêté ou suspendu) en 2018, car le Conseil a estimé que celui-ci utilisait systématiquement des expressions, des phrases et des suggestions sexuelles inappropriées, enfreignant ainsi les normes éthiques et professionnelles (AI-Sayed, 2018). Le Conseil a également obligé la chaîne de télévision qui diffuse le programme satirique "Abla Fajita», à supprimer toute suggestion sexuelle et à préciser le nom de l'acteur qui joue le personnage de la poupée (Fajita), afin qu'il puisse être tenu responsable en cas de manquement à la réglementation en vigueur. Enfin, en février 2021, le Conseil a suspendu l'émission sociopolitique "Akher Al-Nahar» présentée par le journaliste Tamer Amin, accusé d'enfreindre les normes et valeurs morales de la société égyptienne pour avoir offensé les populations de la Haute-Égypte (Al-Ahram, 2021). Enfin, le Conseil exerce un contrôle sur les campagnes publicitaires, en décidant notamment d'interdire la diffusion d'une publicité de l'opérateur Egypt Telecom, sous prétexte qu'elle pourrait nuire aux relations égypto-africaines (Abdel Hamid, 2018).

En définitive, toutes ces décisions sont prises au nom du respect de «la décence publique», une notion suffisamment vague et élastique pour conférer de fait la fonction d'une "police morale» au Conseil suprême de régulation des médias. Ce nouveau rôle est confirmé par la publication, en mars 2019, d'une charte des "codes moraux et des mœurs écrites ${ }^{11}$ ", à laquelle ont été ajoutés d'autres codes en 2021. Ce document du Conseil énumère plus de quatre-vingt-dix codes à respecter, répartis en dix rubriques et concernant: la protection des mœurs, de la morale et le respect des traditions de la société; le contenu religieux; les sujets liés aux femmes; la couverture médiatique des affaires africaines et arabes; la couverture médiatique du terrorisme; la sécurité nationale et économique; les programmes à destination des enfants; la protection de la propriété intellectuelle; l'information sportive; les séries télévisées et la publicité.

\footnotetext{
10 «Saturday Night Light » est une émission sarcastique de divertissement diffusée sur la chaîne On.e (l'ex-chaîne ONTV).

11 La charte est disponible sur le site du Conseil: http://scm.gov.eg/\%d8\%a7\%d9\%84\%d9\%8 5\%d8\%b9\%d8\%a7\%d9\%8a\%d9\%8a\%d8\%b1-\%d8\%a7\%d9\%84\%d8\%a5\%d8\%b9\%d9\%84\%d 8\%a7\%d9\%85\%d9\%8a\%d8\%a9-\%d8\%a3\%d9\%83\%d9\%88\%d8\%a7\%d8\%af-\%d8\%a7\%d9\%84 \%d8\%aa\%d8\%ba\%d8\%b7\%d9\%8a\%d8\%a9-\%d8\%a7\%d9\%84\%d9\%85/
} 


\section{La propriété des chaînes privées et son statut ambigu}

\section{Sous Moubarak, le contrôle par les hommes d'affaires}

Si la relation entre les hommes d'affaires et le régime accordait des privilèges aux premiers, elle n'était pas dépourvue d'outils de «verrouillage». La loi sur l'investissement interdisait par exemple la diffusion de chaînes satellitaires de l'extérieur de la zone franche de la Cité des médias et, par là même, que les chaînes privées possèdent des appareils SNG de transmission directe. À titre d'illustration, Ahmed Bahgat, propriétaire du groupe Dream TV, a obtenu grâce à ses relations privilégiées avec le régime de Moubarak le droit de diffuser les programmes de ses chaînes depuis ses propres studios, via une autorisation présidentielle. De la même manière, lors du lancement de la chaîne ONTV en 2009, I'homme d'affaires Naguib Sawiris avait obtenu une autorisation informelle de diffuser des JT, dérogeant ainsi à la charte de la Cité des médias.

Le contrôle des contenus de ces chaînes de télévision privées passait également à travers des pressions économiques sur les propriétaires. Le cas le plus significatif est de celui d'Ahmed Bahgat au début des années 2000. Les banques lui demandaient de rembourser ses dettes si des cadres du pouvoir estimaient qu'un animateur de l'une de ses chaînes avait dépassé les limites autorisées de la critique. Une autre modalité de contrôle ou de censure des contenus médiatiques est expérimentée à la fin des années 2000 concernant le journal privé al-Dustûr (2005-2010). Il est racheté par deux hommes d'affaires, qui écartent Ibrahim Issa, son rédacteur en chef, jugé trop déviant: «Le journal a été acheté pour le liquider, il n'y a pas d'autres raisons. La preuve c'est que, peu après, un des deux hommes d'affaires a revendu sa part», témoigne sous couvert d'anonymat un ancien journaliste du titre ${ }^{12}$.

\section{Sous Al-Sissi : le jeu des acquisitions et la création des «zones parallèles»}

Les transformations actuelles du paysage audiovisuel égyptien ont eu lieu bien avant la prise du pouvoir par les états-majors militaires en juillet 2013. Elles se sont amorcées avec le lancement entre 2011 et 2012 de chaînes satellitaires qui prônent une ligne éditoriale qu'on pourrait qualifier de

12 Entretien, 2016. 
«contre-révolutionnaire», axée notamment sur la «protection des piliers de l'État», l'armée et la police essentiellement ${ }^{13}$. Ces chaînes de télévision ont été fondées par des hommes d'affaires proches des "autorités souveraines», cette expression désignant en Égypte les différents appareils sécuritaires de l'État. C'est aussi le cas de CBC News, fondée par l'homme d'affaires Mohamed El-Amin, mais surtout de la chaîne Sada al-Balad (L'Écho du pays), lancée en 2011 par Mohamed Abou El-Enein ${ }^{14}$, un homme d'affaires ancien cadre du PND. Bien qu'elles émergent dans un contexte politique transitionnel très fluide, suite aux événements de janvier 2011, ces chaînes s'inscrivent à l'époque dans le cadre de ce que nous pouvons appeler la «zone grise». La dénomination reflète en fait le processus de catégorisation en vigueur durant cette période de la part des activistes et des professionnels des médias qui opposait partisans et non-partisans de la révolution. Elle tend à expliquer le paradoxe que, dès les premiers jours de la création de ces chaînes, elles accueillaient aussi bien les présentateurs et les journalistes connus pour leur ton critique que ceux connus pour leur proximité avec les appareils de l'État, voire ceux affichant une hostilité proclamée à toute forme d'opposition aux institutions. Cela avant que les premiers ne fussent éradiqués de la scène médiatique (Adib Doss, 2018, p. 622).

À partir de 2016, un nouveau paysage audiovisuel se dessine graduellement, dans lequel la propriété directe des chaînes satellitaires est devenu le principal outil de contrôle de l'information, sans pour autant remettre en cause le principe de la propriété privée des médias. Pour le directeur-adjoint de l'Autorité nationale des médias audiovisuels, Gamal Al-Shaeir ${ }^{15}$, ce nouveau paysage répondrait à une logique d'urgence selon laquelle le président Al-Sissi devrait procéder à un changement «rapide et radical » après deux révolutions. Selon lui, les conseillers du président ont insisté sur le fait que les médias doivent constituer un des piliers de ce changement ${ }^{16}$. Dans cette nouvelle configuration, la télévision publique

\footnotetext{
${ }^{13}$ Entretien avec Amr Al-Khayat, le 4 novembre 2014, au siège du quotidien étatique Al-Akhbar. Al-Khayat était directeur des programmes de la chaîne Sada Al-Balad et ex-rédacteur en chef de l'émission «Misr Al-Naharda » (L'Égypte aujourd'hui) à la télévision publique, rédacteur en chef du magazine Akhbar Al Negoum (Les nouvelles des stars), une des publications de l'institution de Al-Akhbar. Il est depuis mars 2021 le directeur de la chaîne Al-Mehwar.

${ }^{14}$ Mohamed Abou El-Einen est un magnat de l'industrie de la céramique. Il fonde en 1983 la société Cleopatra Group, qui monopolise rapidement $60 \%$ du marché local de la céramique. Il est député au parlement depuis 2020.

${ }^{15}$ Entretien, 20 janvier 2021.

${ }^{16}$ Voir aussi la vidéo où Al-Sissi parle en 2013 de l'importance des «bras médiatiques» pour influencer «l'opinion publique»: https://www.youtube.com/watch?v=WB9MVTR02YE
} 
continue d'exister, le pouvoir politique rachète des groupes médiatiques privés en faisant pression sur les propriétaires et transfère la gestion de ces médias aux services de renseignements. Cet espace médiatique qu'on peut qualifier de "sécuritaire ${ }^{17}$ » se construit autour d'alliances non déclarées et très floues entre quelques hommes d'affaires loyalistes (Guaaybess, 2019) et les appareils sécuritaires de l'État.

Dans une certaine mesure, ce nouvel état de l'espace médiatique devait répondre à une nécessité imposée au régime d'Al-Sissi, celle de «la guerre contre le terrorisme». En effet, les chaînes de télévision publiques étant largement discréditées dans la population, le régime politique avait besoin de disposer rapidement de médias capables de la mobiliser pour défendre l'action de l'armée.

La redéfinition de l'espace médiatique actuel s'est donc réalisée peu à peu via ce que nous pourrions appeler «le jeu des acquisitions», tant et si bien qu'en quatre ans le secteur audiovisuel égyptien est monopolisé par une seule société, Eagle Capital, détenue par les services de renseignement. Le schéma 2 réalisé par nos soins ${ }^{18}$ apporte en 2020 une photographie instantanée de ce mouvement de concentration des principaux médias privés du pays, qu'il s'agisse des chaînes satellitaires, des journaux ou des sites web. En mai 2016, l'Egyptian Media Group (EMG) s'est ainsi emparé de la chaîne privée ONTV et de plusieurs titres de presse, avec pour représentant officiel Abou Hashima, un jeune homme d'affaires jusqu'alors peu connu ne détenant alors qu'une part minime des actions. Le groupe a été racheté en septembre 2017 par la société d'investissement Eagle Capital, qui n'a donc pas arrêté de s'approprier les différentes plateformes médiatiques. Eagle Capital est dirigée par Dalia Khorshid, ex-ministre de l'Investissement, conseillère des forces armées pour les affaires financières et épouse de Tarek Amer, l'actuel président de la Banque centrale d'Égypte. À la suite du rachat de I'Egyptian Media Group par Eagle Capital, un nouveau conseil d'administration a été mis en place, présidé par Tamer Morsi, un businessman et producteur de télévision, et Yaser Sélim, un ex-officier des services de renseignement. Le conseil d'administration du bouquet de chaînes DMC, né en septembre 2016 et appartenant désormais à l'Egyptian Media Group,

\footnotetext{
${ }^{17}$ Entretien avec Gamal Al Shaeir, 20 janvier 2021.

${ }^{18}$ Nous avons commencé à réaliser ce schéma en mars 2018. Il a été modifié depuis à plusieurs reprises à cause des continuelles fusions qui se font et se défont et surtout la rapide expansion des acquisitions de la société "Médias des Égyptiens», qui a fini en septembre 2018 par monopoliser la quasi-totalité du secteur audiovisuel.
} 
est également présidé par un ancien officier du service des renseignements de l'armée, Tarek Ismaeil, qui préside aussi l'agence de publicité D-Media. Depuis 2019, Tamer Morsi ${ }^{19}$ supervise également le conseil d'administration d'United Media, une autre société fondée probablement par Eagle Capital qui a annoncé le rachat des parts de l'Egyptian Media Group. L'examen des registres commerciaux des deux sociétés ${ }^{20}$ montre qu'Eagle Capital ${ }^{21}$ est en effet à l'origine de l'Egyptian Media Group, mais son nom ne figure pas sur le registre commercial de la société United Media.

Dans l'espace représenté ci-dessous, les chaînes Al-Mehwar et Sada Al-Balad demeurent toutes les deux officiellement sous la tutelle d'hommes d'affaires. En revanche, le contrôle est exercé via le contenu: Mohamed Al-Baz, connu par sa proximité avec Amn al-dawla, a dirigé par exemple Al-Mehwar jusqu'à mars 2021, remplacé par Amr Al-Khayat, le rédacteur en chef du quotidien public Al-Akhbar. Cette même stratégie est utilisée avec le journal Al-Masry al-Yaoûm, appartenant officiellement à I'homme d'affaires libéral Salah Diab, mais Abdellatif Al-Manawy ${ }^{22}$, qui fait partie de son conseil d'administration, est actuellement son rédacteur en chef par intérim, prenant la suite de Hamdy Rizk, journaliste également proche des «autorités souveraines». (Atef, 2018) ${ }^{23}$

\footnotetext{
${ }^{19}$ Tamer Morsi a été écarté après le ramadan en mai 2021. Les raisons annoncées seraient liées à des questions de corruption et de dépenses non justifiées (Al Sadat, 2021).

${ }^{20}$ Exemplaire du registre commercial Kbis $n^{\circ} 108058 / 2021$ et Kbis $n^{\circ} 92430$, consulté auprès de la Haute autorité de l'investissement.

${ }^{21}$ Nous n'avons pas pu consulter le registre commercial de la société Eagle Capital.

${ }^{22} \mathrm{Al}$ Manawy est une figure polémique du paysage médiatique égyptien. Directeur du secteur de l'Information à Maspero, la télévision publique, de 2005 à janvier 2011, il fut longtemps accusé d'être un des artisans du projet de la succession par héritage du fils du feu président Moubarak. On lui reproche également la couverture médiatique du soulèvement de 2011.

${ }^{23}$ Le paysage est en effet assez fluctuant et les informations qui figurent dans le graphique ont été mises à jour jusqu'en décembre 2020. Des négociations sont par exemple en cours concernant le rachat du bouquet de chaînes d'Al-Nahar par l'Egyptian Media Group et le rachat $\mathrm{d}^{\prime} \mathrm{Al}-\mathrm{Mehwar}$ est à son tour en cours au moment de la rédaction de ce texte.
} 


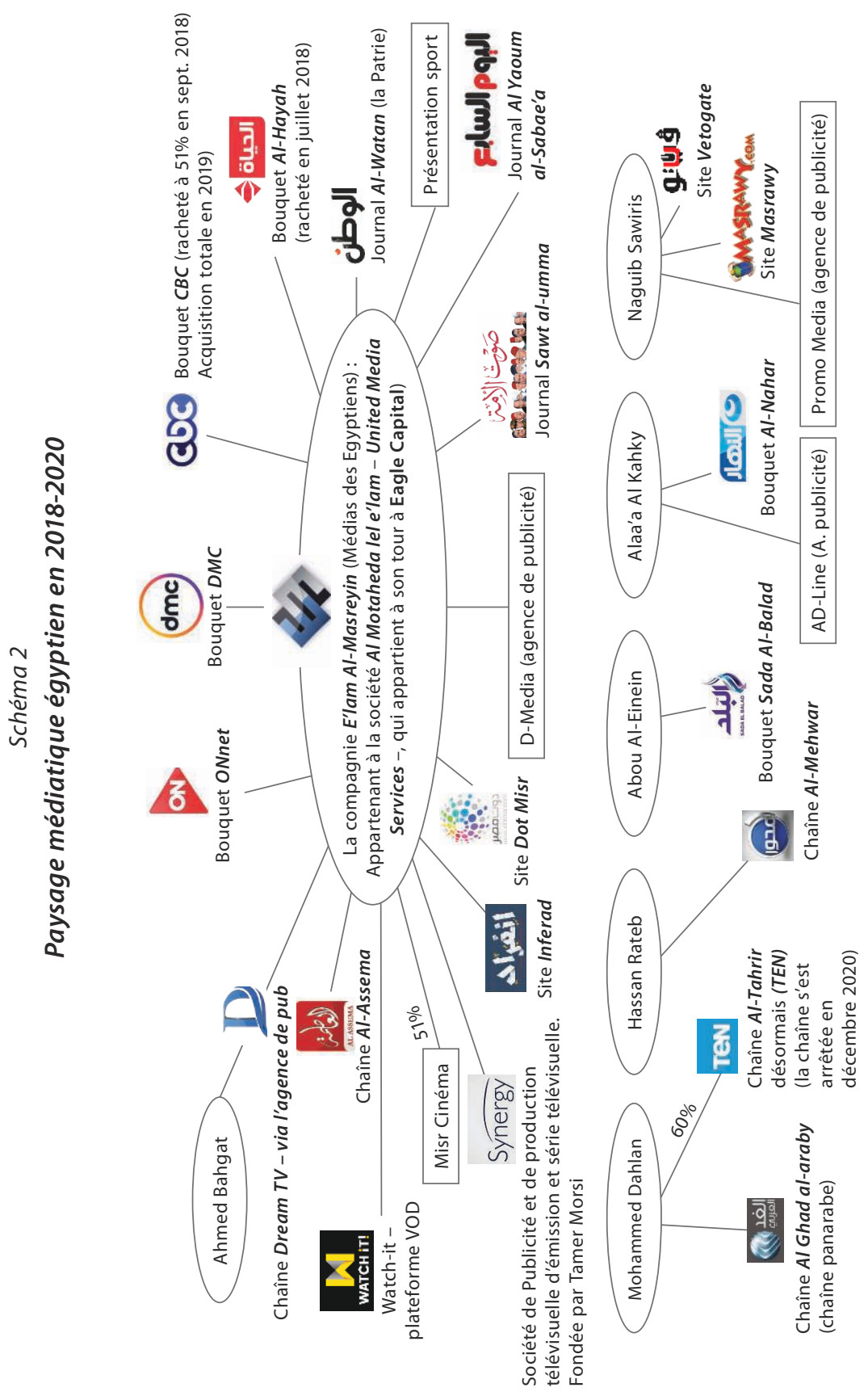




\section{La régulation informelle}

La régulation informelle du secteur audiovisuel en Égypte n'a pas connu d'importantes modifications entre l'ère Moubarak et celle de son successeur. Elle demeure le moyen de contrôle le plus pesant sur le travail journalistique en général et télévisuel en particulier. Toutefois, une nouvelle phase de fermeture de l'espace médiatique émerge après 2013, ces modalités de contrôle devenant à la fois plus explicites, plus étendues et surtout moins négociables.

\section{Des «lignes rouges » insaisissables}

Le journaliste, intellectuel et activiste Hamdy Kandil ${ }^{24}$ définissait ainsi la «ligne rouge» dans son autobiographie:

"La ligne rouge est une ligne imaginaire exactement comme celle de l'équateur terrestre. Or, l'équateur est stable et connu de tout le monde. La ligne rouge, par contre, est changeable d'une chaîne à l'autre et d'un moment à un autre. Elle s'élève et s'abaisse selon les régimes ou selon le thermomètre des événements. Elle oscille avec les oscillations du tempérament des contrôleurs ou des responsables de la censure (...). Dans leurs tentatives de tâtonnement, les présentateurs des émissions souffrent pour découvrir où se trouve la ligne rouge. Ils échouent le plus souvent. Le danger n'est pas dû à cet échec, mais plutôt à la peur que leurs paroles ne dépassent cette ligne. Ainsi, ils n'accompliront pas leur mission.» (Kandil, 2014, p. 414)

En Égypte, les «lignes rouges» sont un amalgame de sujets sans limites ni bornes, difficilement définissables et dont les frontières varient d'une période à l'autre, c'est-à-dire qu'elles dépendent du degré de liberté octroyé par le régime. Parmi les «lignes rouges» les plus fréquemment mentionnées par les journalistes, on peut citer: le président et sa famille, l'armée, les minorités et les problèmes confessionnels, la politique extérieure et les questions à caractère sexuel (Webb, 2014, p. 26-30). Concernant la première, elle a été abaissée vers 2005 avec les amendements constitutionnels et l'introduction du choix du président par l'élection, mais celle-ci a été renforcée à nouveau en 2007

\footnotetext{
${ }^{24}$ Khandil est un journaliste intellectuel engagé, un animateur qui joua un rôle important dans la modernisation du discours télévisuel dans les années 1990. Il fut une figure proéminente du journalisme télévisuel depuis ses débuts. Il a commencé sa carrière de journaliste dans les années 1950 au magazine Akher Sa'a (Dernière heure). En 1961, il animait une émission de revue de presse qu'il commentait, intitulée "Aqwal al-Sohof» (Paroles de presse) pendant près de huit ans jusqu'en 1969, année où il fut nommé directeur de l'Arab States Broadcasting Union (ASBU).
} 
avec la fameuse accusation portée à l'encontre du journaliste Ibrahim Issa de «répandre de fausses informations » concernant l'état de santé du président. C'est ce qui explique aussi le durcissement des sanctions qu'encourait tout média entendant évoquer la question de la succession héréditaire du pouvoir.

La deuxième «ligne rouge » est, d'après Edward Webb, la «ligne la plus rouge» (Webb, 2014, p. 26): les journalistes ne peuvent publier aucune information sur l'armée sans l'autorisation du département des Affaires morales de l'armée ("al-shou'an al-maanaweya»). Cette ligne est restée infranchissable même après la révolution de 2011 puis s'est durcie à partir de 2013. La troisième «ligne rouge » concerne la question confessionnelle et porte en fait sur la minorité copte, son traitement médiatique ne devant jamais sortir d'un certain cadre préétabli. À titre d'exemple, les tensions entre musulmans et chrétiens doivent être mentionnées comme des cas exceptionnels et résultant de comportements individuels, en omettant volontairement toute dimension confessionnelle ou collective. Les questions religieuses sont plus généralement considérées comme très sensibles, et les «religions du Livre» ne peuvent pas être critiquées (Webb, 2014, p. 29).

En effet, ces «lignes rouges » se caractérisent par deux traits. Tout d'abord, elles sont légitimées devant l'«opinion publique» par des métadiscours à caractère propagandiste. C'est le cas par exemple de tout ce qui relève de "l'atteinte à la réputation de l'Égypte» ou à son «image», tout particulièrement à l'étranger. Certaines publications peuvent donc être censurées car elles sont considérées comme antipatriotiques ou parce qu'elles nuisent à "l'image positive du pays». C'est le cas également du "complot étranger» ou plus récemment de «la guerre contre le terrorisme». Quand elles sont dépassées, certaines «lignes rouges» sont jugées comme une "atteinte à la sécurité nationale» et/ou sont perçues comme un «complot étranger» que seules les autorités peuvent résoudre.

Ensuite, le respect des «lignes rouges» s'appuie, en dehors des lois de I'audiovisuel, sur un socle juridique de formulations vagues et extensibles, permettant ainsi diverses interprétations politiques. C'est le cas notamment de la section 14 du Code pénal (58/1937, amendée à plusieurs reprises par les lois 95/2003 et 147/2006), qui est intitulée "Les crimes de la presse» (Amer, 2012, p. 37-40). La loi $n^{\circ} 95$ de 2003 interdit par exemple les publications qui incitent "à la haine du régime», "l'humiliation des autorités civiles», «l'humiliation des forces armées ou du Parlement», «l'excitation de l'opinion publique par la propagande», "la transmission de fausses nouvelles», «l'attaque de l'une des trois religions monothéistes», «la propagation de 
l'athéisme » ou encore «la publication d'informations qui troublent la paix et l'ordre public ». Les peines mentionnées par l'article 80 de la même loi vont jusqu'à la prison à vie si les informations publiées sont parvenues à un État considéré comme ennemi (Amer, 2012, p. 37). Il est également interdit de «promouvoir des idées qui incitent à la haine publique du régime» (article 174 de la section 14 du Code pénal).

L'article 102 de la loi de 2003 pénalise «la publication d'informations, de statistiques mensongères ou de rumeurs qui nuisent ou troublent la sécurité nationale, apeurent le peuple ou nuisent à l'intérêt public en période de guerre ». L'article 178 condamne la publication de tout tract, image ou autre qui porterait atteinte à la réputation du pays ou nuirait à son image. Le plus souvent, c'est en vertu de cet article que les journalistes qui traitent de cas de torture ou de la question des droits de l'hommes sont pénalisés.

L'article 179 interdit aux journalistes «d'insulter le président». Cela peut conduire à l'arrestation du journaliste, à sa mise en garde à vue, voire à une peine de prison de trois ans et à la fermeture du journal. Ainsi, toute critique du président peut être traduite comme une insulte (Amer, 2012, p. 39).

L'article 195 impute la responsabilité au rédacteur en chef du journal pour tout "crime» commis par ceux qui travaillent sous sa direction ${ }^{25}$. À ces différentes dispositions, fortement contraignantes pour la liberté d'expression des journalistes, il faut ajouter également «l'état d'urgence » en vigueur depuis 1967, à l'exception de quelques mois (18 entre mai 1980 et octobre 1981, date de l'assassinat du président Sadate, et entre le 31 mai 2012 et août 2013). L'état d'urgence est sans cesse renouvelé pour une durée de trois ans par approbation du Parlement. Le président exerce son autorité par décret et peut ainsi imposer des restrictions à la liberté de réunion, de mouvement et de résidence, ordonner l'arrestation et la recherche des personnes suspectes qui constituent une menace pour la sécurité. En outre, le même texte prévoit la condamnation des civils devant les tribunaux compétents en cas d'atteinte à la «sécurité de l'État», soit pour des violations de la «loi d'urgence» elle-même, soit pour des décrets promulgués en son nom, ou encore pour des infractions pénales ordinaires. Le président exerce également un veto sur toute décision du Tribunal de sécurité de l'État.

\footnotetext{
${ }^{25}$ Le Code pénal autorise la confiscation des documents écrits s'ils sont utilisés pour commettre ou inciter à certains «crimes» tels que l'obscénité ou l'intention de renverser le gouvernement. Cependant, la confiscation doit suivre une procédure régulière. Si la police soupçonne qu'un tel crime a été commis par la presse, elle doit porter l'affaire devant le procureur, qui prend la décision de s'en saisir ou non.
} 


\section{L'emprise de "l'État des services de renseignement "}

Les modes informels de fermeture de l'espace médiatique renvoient également à ce que I'universitaire Philippe Droz-Vincent a appelé "l'omniprésence de dawlat al-mukhabarat» (I'État des services de renseignement) (Droz-Vincent, 2004, p. 948), c'est-à-dire l'emprise exercée par les différents appareils de renseignement ou les différentes «autorités souveraines» sur la vie politique et sociale du pays. Historiquement, la police politique instaurée sous l'occupation pour poursuivre les opposants au pouvoir anglais au nom de la sécurité de l'État n'a jamais cessé d'exister. Sous la république, après l'indépendance, le service en question, désormais appelé Amn-Al-Dawla (service d'investigation de la Sécurité de l'État) visait avant toute chose la préservation de l'existence du régime politique ${ }^{26}$. Elle fonctionne parallèlement à deux autres services de renseignement: les renseignements extérieurs et les renseignements militaires.

Quant aux médias, l'ampleur des modalités de contrôle exercés par ces instances sécuritaires, entre méthodes dures et autres plus souples, varient en fonction du type de support, mais également selon les périodes et la «logique de gestion» en vigueur: concrètement, cela oscille entre les appels téléphoniques et la présence physique d'un agent dans les locaux d'une chaîne.

\section{Communiquer les consignes: du contact direct à WhatsApp}

La prise de contact pour recevoir les directives et le cadrage à adopter pour certains récits médiatiques est une pratique assez ancienne. Le but étant d'éviter toute déviation susceptible d'adresser des critiques virulentes contre le régime politique, la stratégie consiste souvent soit à bloquer l'information jugée suspecte par les autorités souveraines soit à définir le cadre de prépublication. Les méthodes concernant les journalistes et animateurs n'ont cessé d'évoluer.

Le politiste Ma'amoun Fandy revient sur les activités de contrôle exercées sur la presse par le bureau de censure du ministère de l'Intérieur (Fandy, 2007, p. 30). Cette domination se manifeste ainsi, pour les médias publics, la presse et la télévision, par une prise de contact quotidienne pour s'assurer du cadrage des principaux récits et recevoir les instructions concernant les «lignes rouges». C'est ce qu'Edward Webb appelle aussi «the constant

\footnotetext{
${ }^{26}$ Le service a changé de nom en 2011, remplacé par Al-Amn Al-Watany (Service de la sécurité nationale).
} 
phone calls » pour renseigner les consignes avant la publication ou bien pour exprimer un mécontentement, parfois du ministre de l'Intérieur lui-même, post-publication (Webb, 2014, p. 45).

Avec l'introduction des chaînes privées, la modalité de contrôle a pris une nouvelle forme. Un rédacteur d'un programme de la chaîne satellitaire Dream TV, sous Moubarak, résume bien la méthode du blocage à la source:

"Quand notre talk-show était hebdomadaire et que les émissions étaient enregistrées, il y avait un délégué des services d'Amn al-dawla, comme c'était le cas pour les autres chaînes. Dans notre cas, il faisait des montages, et les choses qu'il estimait indésirables ne passaient pas. Une fois, ils ont refusé la diffusion d'une émission complète parce qu'on traitait le dossier confessionnel (...). Dès lors que nos émissions sont devenues quotidiennes et en direct, donc difficiles à censurer, les moyens ont changée ${ }^{27}$.»

Toujours sous Moubarak, les appels téléphoniques ou les hotlines entre le bureau responsable du suivi des talk-shows diffusés sur ces chaînes étaient des pratiques récurrentes. Quand des membres du bureau de la sécurité d'État estimaient que l'animateur ou l'invité avaient dépassé un certain seuil de tolérance, ils contactaient le directeur de la chaîne ou l'équipe de rédaction, le directeur de la programmation de la chaîne ou même le propriétaire pour arrêter «la mascarade immédiatement», comme l'explique en entretien un rédacteur en chef d'une émission télévisée. Plusieurs présentateurs ont ainsi vu leur émission coupée en direct, sous prétexte de problèmes techniques ${ }^{28}$.

Certains agents de ces services ont, semble-t-il, une perception négative du rôle des médias, selon Mona Al-Shazly, célèbre animatrice de talk-show. Dans un entretien, elle a évoqué les appels téléphoniques qu'elle recevait constamment pour lui dicter ce qu'elle avait ou non le droit de faire et de dire ou bien pour s'indigner des critiques dont leurs auteurs avaient été l'objet. Elle faisait ainsi remarquer que le régime et ses institutions avaient du mal à comprendre la différence entre une couverture médiatique et une hostilité personnelle. Selon elle, c'est une relation «qui cherche toujours sa boussole».

«Un responsable officiel du service de sécurité m’a appelée il y a quelques semaines et m'a demandé sans transition aucune: "Pourquoi tu nous hais ainsi ?" Moi franchement, je n'ai pas compris ce qu'il entendait par-là (...).

\footnotetext{
${ }^{27}$ Entretien avec un rédacteur de l'équipe d'une émission de la chaîne Dream, en 2015.

${ }^{28}$ Entre autres l'émission «Sabah Dream» (Bonjour Dream) animée par Dina Abdel Rahman pendant les 18 jours du soulèvement de janvier 2011.
} 
Il m'a expliqué qu'il pensait que je haïssais personnellement la police et les forces de sécurité du ministère de l'Intérieur (...). C'était une longue conversation, j'ai essayé de lui expliquer ce que nous faisions, en vain. Ça prouve que la boussole est toujours absente ${ }^{29}$.»

Ce témoignage rejoint l'analyse de Ma'moun Fandy qui qualifie de "news receiving" ce genre de relation instaurée entre le régime et les journalistes (Fandy, 2007, p. 10). Pendant longtemps, une grande partie des informations gouvernementales ont été rédigées non pas par des journalistes, mais par des agents des bureaux des Elaqat al-ama (relations publiques) du président, des gouverneurs, des ministres ou encore d'Al sheoun al ma'naweya (le département des affaires morales ${ }^{30}$ ), qui est le service responsable des médias et de la morale des Forces armées égyptiennes. Ces déclarations sont généralement publiées telles quelles, notamment dans les journaux officiels. Les relations entre la presse et l'opposition reproduisent le même schéma : «il s'agit de recevoir des nouvelles plutôt que de rassembler », explique Ma'moun Fandy (Fandy, 2007, p. 10). S'assurer de l'authenticité de l'information et, en particulier, de celle provenant de ces bureaux n'est pas une pratique courante.

Autrement dit, cette même configuration à la télévision se retrouve sous Al-Sissi. C'est ce qu'a exprimé Gamal Al-Shaeir en qualifiant les productions des médias égyptiens à I'heure actuelle: «Ce que nous voyons sur les écrans de télévision ne sont pas des informations mais des rapports pré-fournis ${ }^{31}$.» Les illustrations de ce mode de fonctionnement sont en effet multiples. Après juillet 2013, toutes les chaînes ont reçu une liste de dix personnes désignées comme des «experts stratégiques ${ }^{32}$ ». Dès lors que les journalistes et animateurs abordent des questions liées à l'armée, les émissions doivent obligatoirement choisir des invités dans cette liste. II s'agit essentiellement d'anciens militaires à la retraite qui maintiennent des relations privilégiées avec l'armée et dont les propos sont donc en quelque sorte négociés en amont puisqu'ils représentent forcément le discours officiel de l'armée.

\footnotetext{
${ }^{29}$ Propos de Mona AI Shazly, émission «De Washington », sur la chaîne Al Jazeera, le 7 décembre 2016. Transcription de l'émission. URL: http://aljazeera.net/home/print/0353e88a-286d-4266-82c66094179ea26d/cab96159-fee3-401d-9962-cfb4d4461c29

${ }^{30} \mathrm{C}$ 'est le département responsable des médias et des affaires morales et psychologiques des Forces armées égyptiennes.

${ }^{31}$ Entretien avec Gamal Al-Shaeir, 20 janvier 2021.

32 La liste mentionnée à plusieurs reprises nous a été communiquée par un rédacteur en chef sous couvert d'anonymat. Les personnes citées sont tous des généraux à la retraite : Sameh Seif Al Yazal (décédé en 2016), Abdel Moneim Said, Hamdy Bekhit, Nasr Salem, Mohamed Mokhtar Kandil, Ahmed Abdel Halim, Kamal Amer, Ali Hefzy, Nabil Fouad, Talaat Moussa.
} 
Pendant de longues années, et même après janvier 2011, le responsable des médias au ministère de l'Intérieur a été le général Alaa Mahmoud (Shaltout, 2012), directeur du «Bureau de production médiatique du ministère de l'Intérieur» et responsable de la couverture de ce ministère. II était appelé par les professionnels des médias "l'homme fort du ministère ${ }^{33}$ ॥. II avait été le garde du corps de Safwat Al-Shérif, un des principaux responsables du régime égyptien depuis l'instauration de la République en 1952 et un des fondateurs du département d'espionnage d'Al-mukhabarat al-amma ${ }^{34}$.

«La relation avec le bureau de Alaa Mahmoud était tendue. Il détestait les chaînes privées et nous considérait comme faisant partie du plus grand complot. Une fois, un de nos photographes a été arrêté alors qu'il filmait la police dans la rue. Lorsqu'ils ont su qu'il faisait partie de l'équipe de notre chaîne, nous fûmes surpris quand, le lendemain, une fille porta plainte contre lui pour l'avoir incitée à la délinquance. C'était une accusation dangereuse, car ce faisant, on nous accusait de porter atteinte à la sécurité nationale.» (Entretien avec un journaliste d'une chaîne de télévision privée, sous couvert d'anonymat en 2020)

«Le bureau faisait en sorte que nous (toutes les chaînes) soyons dépendants de ce qu'ils nous fournissaient. Nous avons joué le jeu. Ils nous fournissaient des vidéos que le ministère produisait sur l'activité de la police: des arrestations de personnes armées, des passages de patrouilles, etc. Mais ils faisaient aussi parfois des vidéos ou des récits en exclusivité pour une chaîne, pour l'apprivoiser, et la fois d'après c'était le tour d'une autre (...), et de temps en temps, ils invitaient nos équipes à descendre filmer avec eux avec nos caméras.» (Entretien avec un journaliste d'une chaîne de télévision, sous couvert d'anonymat, 2016)

\footnotetext{
${ }^{33}$ Cette dénomination nous a été répétée par plusieurs interviewés lorsqu'il était question de cette personne.

${ }^{34}$ Safwat Al-Sharif a rejoint en 1957 le service des renseignements généraux, Al Mukhabarat al-amma sous Nasser. Il est considéré comme l'un des fondateurs du département d'espionnage, recrutant et formant toute une génération dans ce département. En 1968, il est placé en résidence surveillée lors d'une enquête sur d'éventuelles dérives du service, mais il est finalement libéré. Entre cette date et 1957, il établit un commerce d'exportation et d'importation qu'il ferme pour rejoindre le Service d'information étatique qu'il préside par la suite. En 1977, il est membre fondateur du Parti national démocrate (PND), et, depuis, il a toujours été membre du Bureau politique du parti. Le président Anwar El-Sadate l'a nommé ensuite président de I'Union égyptienne de la radio et de la télévision en 1980. En 1982, il est nommé ministre de I'Information par Hosni Moubarak jusqu'en 2004, devenant par ailleurs, en septembre 2002, secrétaire général du PND. Depuis 2004, Al-Sharif occupe le poste de président du Conseil Shura (la chambre haute du Parlement) et de président du Conseil suprême de la presse, qui octroie les licences aux nouveaux journaux. Il a démissionné de ses fonctions en janvier 2011.
} 
Cette pratique a été également appliquée à une très grande échelle à peu près depuis 2015. Les directives et les sujets à traiter ainsi que les axes autorisés seraient distribués via des groupes WhatsApp incluant les rédacteurs en chef des chaînes ou certains animateurs ${ }^{35}$. L'uniformisation des contenus résultat de cette pratique est tellement saillante que la moindre fausse note suscite l'étonnement. Le commentaire d'un journaliste sur sa page Facebook en réaction aux propos d'un animateur critiquant la hausse des prix le montre: «WhatsApp a planté hier ou quoi?» ${ }^{36}$.

\section{Les méthodes dures: entre recrutement et menaces}

La Sécurité de l'État, la police politique du ministère de l'Intérieur (désormais appelée la Sécurité nationale) ont une longue tradition d'ingérence dans le travail des journalistes, recrutant les uns pour travailler comme informateurs ou propagandistes et/ou intimidant les autres en les menaçant d'une peine de prison ou de nuire à un membre de leur famille ${ }^{37}$. Ils peuvent aller jusqu'à leur proposer des avantages matériels en échange de leur «coopération ». Certains de nos interviewés ont également affirmé avoir reçu des offres de promotion diverses, des villas ou même des offres de facilités financières pour la publication d'un journal. Leurs propos montrent souvent à la fois une relation tendue et l'expression d'une certaine frustration.

"Avant de recruter des journalistes, "ils" les font passer par une phase d'enquête. Ils discutent d'abord pour voir de quelle manière ils pourront les recruter ou non afin de chercher le moyen adéquat (...). Ils créent même des héros devant l'opinion publique. Ils persécutent, voire mettent en prison des journalistes qu'ils ont déjà recrutés afin de les victimiser et les rendant ainsi plus crédibles. Ils passent ainsi pour des opposants alors qu'ils travaillent en fait à leur service, voire directement selon leurs indications (...). Moi, par exemple, j'étais sûr qu'ils n'allaient pas me persécuter pour éviter que je ne devienne un héros (...). Je note que, dans notre métier il est légitime d'avoir de bonnes relations avec les services de sécurité, ce sont nos sources. Par contre, ce qui ne l'est pas, c'est quand on devient un outil de leur propagande.» (Entretien avec un rédacteur en chef d'une émission de télévision en 2016)

\footnotetext{
${ }^{35}$ Entretien, 2020.

${ }^{36}$ Page Facebook personnelle d'un journaliste de la télévision publique consultée en juin 2021.

${ }^{37}$ Certains de nos interviewés ont affirmé avoir reçu des menaces.
} 
C'est aussi ce qu'a affirmé le journaliste Hisham Qassim: «Ils essaient toujours de vous intimider ou de vous favoriser» (Webb, 2014, p. 38). II a été convoqué au bureau de la Sécurité d'État pour la première fois en 1990 et à plusieurs reprises depuis. Ces manœuvres ne différaient pas pour la télévision.

Cet héritage de nombreuses années de manipulation des journalistes par le pouvoir a eu deux conséquences. D'une part, il a créé un autre niveau de censure, une auto-censure «devenue profondément enracinée dans la culture de nombreux médias » selon Toby Mendel, avocat spécialisé dans les droits de l'homme (Webb, 2014, p. 39). En dépit de leur instabilité, certaines limites et «lignes rouges» sont intériorisées par ceux qui travaillent dans le domaine. D'autre part, cet héritage a créé une atmosphère de suspicion permanente dans l'espace journalistique. Si cette tendance a aussi fortement marqué la période post-janvier 2011, les personnes interviewées ont confirmé qu'elle existait bien avant: «Le fait que ce n'était pas explicite n'empêche pas que, dans le milieu, on savait qui travaillait pour eux», explique ainsi un rédacteur en chef. D'autres, sous couvert d'anonymat, ont confirmé en entretien, allant jusqu'à citer les prénoms des journalistes et animateurs en question: "X est appelé l'agent non-secret du ministère de l'Intérieur par tous les journalistes»; «il y a des journalistes-espions qui écrivent des rapports contre leurs collègues et qu'ils font remonter à $A m n$ al-dawla »; «il faut consacrer une étude entière à la question de la relation des journalistes avec les services de renseignement qui a vraiment marqué notre profession »; «mon travail à Maspero m'a confirmé une chose, rien ne peut se faire sans leur approbation, et tous ceux qui prétendent être intègres et indépendants ne le sont pas». Un rédacteur en chef raconte également sous couvert d'anonymat:

«J'ai été convoqué à Amn al-dawla une fois, ils essayaient de me recruter, bien sûr. Mais en sortant j'ai demandé franchement au gars: qui, parmi les professionnels des médias, travaille avec vous? II m'a répondu que tous ceux qui affirment travailler avec nous ne travaillent en fait pas pour nous. (...) C'est une stratégie de leur part pour nous faire sentir que tous ceux qui nous entourent sont des agents secrets des services de renseignement.» (Entretien, 2020)

Les prises de contact étendues des services de sécurité avec les personnels des médias révèlent des capacités de suivi de l'actualité très développées. Nombre d'interviewés, qu'ils soient simples invités, directeurs de chaîne, journalistes, rédacteurs en chef d'une émission ou propriétaires d'une société de production, ont affirmé avoir été contactés par un des services de sécurité pour commenter une émission ou la présence d'un 
invité. «C'est généralement un officier ou deux qui te prennent en charge. Tu entends souvent le même nom. Ils cherchent ton numéro et après ils ne te lâchent pas. Et Amn al-dawla n'appelle pas n'importe qui », explique le rédacteur en chef d'une émission. Certains interviewés racontent des exemples d'échanges qu'ils ont eus avec leurs interlocuteurs de Amn aldawla. Deux exemples typiques permettent de mieux restituer cette relation.

"Vous avez invité monsieur X ou $Z^{38}$, s'il vous plaît faites en sorte qu'il ne soit plus invité. Nous sommes toujours à votre service et nous savons que vous êtes patriote et c'est pour le bien et la sécurité du pays que nous vous demandons cela. Vous comprenez les choses.»

«Vous avez signé un contrat avec monsieur (I'animateur X), s'il vous plaît nous souhaitons qu'il ne parle plus à la télévision. Nous comptons sur votre patriotisme.

- Oui, mais vous demandez d'arrêter de travailler, mais j'ai des responsabilités à l'égard de mes employés et j'ai des loyers de studios à payer, ce n'est pas possible.

- Nous vous enverrons la chaîne (X) qui louera le studio pendant deux ans et vous serez donc tranquille de ce côté-là, et vous pourrez donc assumer vos obligations.

- Je suis d'accord, mais envoyez d'abord la chaîne dont vous parlez, et après nous verrons, je ne peux rien annuler avant d'avoir une preuve.

- Vous n'avez donc pas confiance en nous?

- Je ne peux pas arrêter un projet sans quelque chose de tangible.»

Selon l'auteur de ce duel, le service a considéré que son refus préalable était un affrontement ${ }^{39}$. II faut préciser que la scène politique égyptienne ne reflète pas toujours la cohérence entre les différents appareils secrets ou services de renseignement. Toute la subtilité de "l'État des services de renseignement» déjà mentionné réside dans ces relations ambiguës. La relation privilégiée d'un journaliste avec l'un ou l'autre des différents services n'implique pas qu'il soit apprécié et protégé par les autres. C'est ce qui explique que la détention ou même les avertissements que peuvent recevoir des journalistes bien connectés pourront tout aussi bien venir d'un service/d'une instance différente de celle avec laquelle le journaliste en question est en bonne relation. En termes d'image publique positive, ces manœuvres demeurent en fin de compte favorables aux journalistes eux-mêmes au sens où ils passent ainsi pour des professionnels discriminés et des opposants intègres.

\footnotetext{
${ }^{38}$ Des figures de l'opposition.

${ }^{39}$ Entretien sous couvert d'anonymat en 2020.
} 


\section{Conclusion}

En fait, l'évolution de la scène politique et médiatique égyptienne en 2021 et le paysage actuel sous Al-Sissi prouvent que le secteur médiatique reste fortement tributaire non seulement du pouvoir politique en place, mais surtout de la conception des autorités sur le rôle et la place que doivent occuper les médias dans la vie politique du pays. L'espace médiatique égyptien a subi d'importantes mutations qui ont débuté par le rachat des médias privés qui avaient connu leurs «heures de gloire» durant la dernière décennie du règne de Moubarak. II s'est ensuivi l'introduction de dispositifs juridiques et la multiplication des instances de contrôle, avec un réinvestissement dans les modalités informelles classiques. Cette configuration permet de comprendre pourquoi et comment la parole publique est confisquée et les contenus médiatiques uniformisés.

\section{Bibliographie}

ADIB Doss Maria, Les talk-shows en Égypte. D'un dispositif de modernisation de l'autoritarisme à des arènes de parole dissidente. Mise en perspective d'une situation révolutionnaire (25 janvier-11 février 2011), thèse en science politique (dir.: Jean-Baptiste Legavre), Université Paris 2, 2018.

Allal Amin, Vannetzel Marie, «Des lendemains qui déchantent? Pour une sociologie des moments de restauration », Politique africaine, $n^{\circ}$ 146, 2017. DOI: https://doi.org/10.3917/polaf.146.0005

AMER Fathy Hussein, Hurreyat al e'alam wa al-sehafa (la liberté médiatique et la loi), Le Caire, Al-Arabi, 2012.

BAYART Jean-François, «Retour sur les Printemps arabes», Politique africaine, $n^{\circ} 133,2014$. DOI: https://doi.org/10.3917/polaf.133.0153

FANDY Ma'moun, (Un)Civil war of words: Media and politics in the Arab World, London, Praeger Security International, 2007.

DROZ-VINCENT Philippe, "Quel avenir pour l'autoritarisme dans le monde arabe?», Revue française de science politique, vol. 54, n 6, décembre 2004.

GUAAYBESS Tourya, "Le champ médiatique comme prisme analytique d'un régime politique», Revue française des sciences de l'information et de la communication, $\mathrm{n}^{\circ}$ 18, 2019, DOI: https://doi.org/10.4000/rfsic.8374

GUAAYBESS Tourya, «Reforming Egypt's Broadcasting in the Post 25 January Era: the Challenges of Path Dependence», dans National Broadcasting 
and State Policy in Arab Countries, sous la dir. de T. Guaaybess, Londres, Palgrave Macmillan, 2013. DOI: https://doi.org/10.1057/9781137301932_5 ISKANDAR Adel, "Defying Definition: Toward Reflexivity in "Arab Media" Studies», dans Media Evolution on the Eve of the Arab Spring, sous la dir. de L. Hudson, A. Iskandar, M. Kirk, New York, Palgrave Macmillan, 2014. DOI: https://doi.org/10.1057/9781137403155_16

KANDIL Hamdy, E'echt maratayn (J'ai vécu deux fois), Le Caire, Al-Sherouk, 2014.

WeBB Edward, Media in Egypt and Tunisia: From Control to Transition?, New York, Palgrave Macmillan, 2014.

\section{Sources}

ABBAS Muhammad, "Al-Sissi : Abdel Nasser kan mahzouz» (Al-Sissi : Abdel Nasser a eu de la chance), Al Masry al Yaoum, 5 août 2014. https://www. almasryalyoum.com/news/details/495094

ABDEL HAMID Ashraf, "Masr tahzor bath e'lan yousi'e il sheoub afrekya " (L'Égypte interdit la diffusion d'une publicité offensante pour les peuples africains), Al-Arabiya, 24 mars 2018. https://urlz.fr/gvPL

AL AHRAM, "Al magles al a'la yokarer wakf bernameg Tamer» (le Conseil suprême décide de suspendre Tamer Amin), Al Ahram, 19 février 2021. https://urlz.fr/f8ID

AL SADAT Mohamed, "Fasl al nehaya: kesat al-etaha be Tamer Morsi wa regaloh » (le dernier chapitre: I'histoire de Tamer Morsi et de ses hommes écartés), Al-Manassa, 20 mai 2021. https://almanassa.com/ar/story/16209 (en arabe)

AL SAYED Muhammed, "Al Majlis Al a'ala lel elam yokarar ikaf SNL bel arabi wa ehalet al nas el helwa lel tahkik» (le Conseil suprême suspend SNL en arabe et le renvoi de la présentatrice de l'émission Al Nas El Helwa pour une enquête), Al Youm Al Sabei, 11 février 2018. https://urlz.fr/f8lw

Atef Magued, "Mostalah al-ajheza al-seyadeya yahdor fe kol sejal (...) la elakat al-amn wa al-e'alam fe Misr» (l'expression "autorités souveraines» est présente au niveau de tous les débats (...) la nature de la relation entre les services de sécurité et les médias en Égypte), Raseef22, 5 septembre 2018. https://raseef22.com/politics/2018/09/05/-مصطلح-الأجهزة /السيادية-يحضر-في-كل-سمجا

BAHJAT Hossam, "Tafasil estehwaz al-mokhabarat al-amma ala e'lam al masreyin» (les détails de l'acquisition de médias égyptiens par les 
services de renseignement), madamasr.com, 20 décembre 2017.

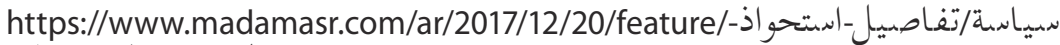

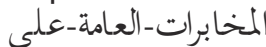

GHALY Mina, "Al a'ala lel e'lam: 200 alf gineih gharama ala al-lafz al mosi'e bel fadaeyat » (le Conseil suprême: 200 mille livres d'amende pour des mots offensants), Al Masry Al Yaoum, 7 juin 2017. https://www.almasryalyoum. com/news/details/1145439

HOSSNI Hager, "Takrir al a'ala lel e'lam an mosalsalat wa barameg ramadan: eha'at gensya wa eskatat seyasya» (le rapport du Conseil suprême concernant les séries et les émissions du mois de ramadan: des références sexuelles et politiques), Masrawy, 7 juin 2017. https://urlz.fr/gvPJ

SHALTOUT Tarek, «Alaa Mahmoud ... asrar wa hekayat akhtar ragol fe wezaret dakhelya (Alaa Mahmoud, les secrets de l'homme le plus dangereux du ministère de I'Intérieur), Al Mogaz, 12 avril 2012. http://www.elmogaz. com/node/23321 


\section{Chapitre 6 \\ Une «libéralisation » économique, politique... et médiatique \\ Les conditions de l'émergence de deux journaux économiques marocains à partir des années 1990}

Dominique Marchetti

Ce chapitre se propose de restituer les conditions de l'émergence à partir des années 1990 de deux importantes entreprises de presse privées (ÉcoMédias et Impression presse édition) non partisanes au Maroc et de leurs cadres dirigeants, qui éditent respectivement les quotidiens économiques L'Économiste (1991) et La Nouvelle Tribune (1995). Elles incarnent parmi d'autres le développement de l'information économique et politique se voulant plus "objective» et "indépendante » à l'égard du champ politique (Benslimane, 2015; Bensmain, 2015). En effet, elles ont contribué, avec d'autres, à transformer un espace journalistique qui jusque-là réfractait stricto sensu l'univers politique, se divisant entre la presse «officielle» et les quotidiens de partis politiques, dirigés par leurs chefs.

L'objet ici n'est pas de déterminer s'il s'agit là des premiers «journaux indépendants» au Maroc, cette histoire étant souvent racontée à travers l'avènement de newsmagazines comme Le Journal -Assahifa (Benslimane, 2015) et TelQuel respectivement en 1997 et 2001. La «libéralisation politique» marquée par «l'alternance» politique avec l'arrivée inédite d'un Premier ministre issu de la gauche (Abderrahman Youssoufi, entre 1998 et 2002) aurait alors incarné la "libéralisation économique». Cette dynamique, encouragée par quelques membres du Palais royal, selon plusieurs enquêtés ${ }^{1}$, aurait alors incité des directeurs de journaux à s'autoriser plus

\footnotetext{
${ }^{1}$ Entretiens avec d'anciens dirigeants du Journal et de TelQuel, octobre 2015 et avril 2016.
} 
de liberté, notamment pour transformer l'«image du Maroc à l'étranger» marquée par les «années de plomb» et préparer la succession du roi Hassan II.

Loin de rentrer dans des débats normatifs, les deux études de cas proposées ici permettent tout d'abord de restituer les conditions de l'avènement de l'entreprise de presse au Maroc dans tous les sens du terme, celles-ci restant encore très peu documentées (Roussillon, 2000; Kraemer, 2001 ; Ait-Mous et al., 2014). Si les projets de L'Économiste comme de La Nouvelle Tribune sont éditoriaux - cet aspect ne sera pas traité dans ce chapitre - et portés en grande partie par des journalistes, ils s'inscrivent également et ouvertement dans des logiques de rentabilité commerciale (Benabid, 2018, p. 256-257): «Notre objectif, comme celui de nos actionnaires, est de gagner de l'argent », explique Nadia Salah à l'origine de la création de L'Économiste (Kraemer, 2001, p. 59). En s'appuyant sur un actionnariat familial, qui permet de contrôler la société, complété par plusieurs membres des fractions dominantes du champ du pouvoir, ce qui "sécurise» le projet, les deux journaux francophones ont jusque-là assuré leur rentabilité. Celle-ci provient essentiellement des recettes publicitaires et d'un lectorat composé des fractions urbaines de l'espace social les plus dotées en capital économique et culturel. Enfin, comme la plupart des titres de presse marocains, ce projet éditorial et économique est indissociablement politique, au sens où il s'agit de défendre, d'un côté, la "libéralisation ", la «modernisation» économique et politique du Maroc et, de l'autre, les institutions, «les valeurs» et l'intérêt de la nation (L'Économiste, 2021).

Ces deux expériences d'entreprise de presse du début des années 1990 s'inscrivent dans un processus de «libéralisation » médiatique contrôlé (Ksikes, 2014, p. 27), qui est marqué par la naissance ou la transformation de quelques sociétés notamment liées à des titres de la presse francophone. Le développement d'Éco-Médias (1991), avec l'hebdomadaire L'Économiste, et de la société Impression Presse Édition (1995), qui édite l'hebdomadaire La Nouvelle Tribune, s'accompagne de celui du groupe Caractères (1990): celui-ci rachètera en 1997 le premier titre spécialisé dans le domaine, La Vie économique, fondé en 1957, propriété du Français Jean-Louis ServanSchreiber depuis 1994 et dont il était actionnaire majoritaire dès 1991 (Kraemer, 2001). D'autres titres économiques vont suivre, comme le raconte Alain Roussillon (Roussillon, 2000, p. 147) dans un article s'interrogeant sur «l'irrésistible engouement qui s'est emparé de cette presse, en particulier francophone, toutes appartenances politiques et idéologiques confondues, pour l'économie, la finance, les marchés... au point de susciter la création 
de plus de soixante titres spécialisés ». L'économie commence également à prendre une place relativement importante dans le quotidien L'Indépendant (1996) et sa version arabophone hebdomadaire Al Moustaqil. À cela s'ajoute la création d'autres sociétés privées à vocation plus généraliste comme celle de l'éditeur du newsmagazine d'information générale et politique Maroc Hebdo en 1991. C'est aussi à cette période, plus précisément dès 1989, que $2 M$, la première chaîne de télévision payante privée et commerciale, se développe à travers la holding royale de l'époque, I'ONA, et plusieurs sociétés étrangères (les groupes français TF1 et Sofirad avec le groupe canadien Videotron). L'une de ses émissions, "Entreprendre " «donnent une voix et une image à "l'entrepreneur" " (Catusse, 2008, p. 11). Cette presse économique strictement francophone est portée par le développement de la publicité pour les classes supérieures urbaines fortement dotées en capital économique. Ce marché est complètement contrôlé par un oligopole à frange, quelques grandes compagnies publicitaires se partageant l'essentiel des campagnes publicitaires des grandes entreprises publiques et privées et des fondations: Shem's, fondée et présidée par le chef d'entreprise Noureddine Ayouch depuis 1972; Klem, créée en 1976 devenue Klem Euro RSCG à la fin des années 1980 et détenue à parts égales par son fondateur, Kadiri Abdelhamid, ancien du groupe Havas, et le groupe français Euro RSCG; Saga Communication, qui voit le jour en 1993 sous l'impulsion de Chakir Fassi Fihri, ancien directeur central à la Banque marocaine du commerce extérieur; DDB Zone Bleue, créée en 1995, filiale du premier groupe mondial de publicité, Publicis-Omnicom; Mozaïk, fondée la même année par Monique Elgrichi, ancienne salariée de l'agence Klem Euro RSCG.

La «libéralisation » médiatique relative incarnée par la presse économique est rendue possible grâce à un processus plus général et progressif de «libéralisation » économique et politique du Maroc à partir de la fin des années 1980 et le début des années 1990. Comme l'ont montré de nombreux travaux, ce processus renvoie avant tout aux injonctions internationales de «libéralisation» des économies et des échanges. À l'instar d'autres nations dont l'économie est fortement dépendante de quelques champs nationaux étrangers et en raison d'une forte crise économique, le Maroc a eu alors recours au Fonds monétaire international (FMI) dès le début des années 1980 et s'est engagé dans un plan dit «d'ajustement structurel » (voir notamment El Malki, 1985), marquant une entrée progressive et avec ses spécificités nationales dans l'«âge néolibéral » (Hibou, Tozy, 2020, $3^{\text {e }}$ partie). Si la montée des exigences internationales est aussi en relation avec les droits de l'homme - la création du Comité consultatif des droits 
de l'homme en 1990, la libération de prisonniers politiques en 1991 et la Constitution de 1992 en témoignent, entre autres -, elle signifie aussi et surtout des injonctions de "libéraliser » l'économie ${ }^{2}$. Certains cadres au pouvoir au Maroc se convainquent de la nécessité (Bennani-Chraïbi, 1997) de développer une image d'un pays allant vers la «modernité libérale», la «transition démocratique» pour attirer les investissements étrangers et les touristes (Hibou, Tozy, 2002), la «liberté de la presse » étant un des éléments de ce discours. Ce contexte est résumé par un des principaux dirigeants de presse marocains.

«Le Maroc a appliqué l'article 8 des statuts du Fonds monétaire international. C'est un tournant important, c'est-à-dire la possibilité d'exporter ses capitaux, la possibilité d'investir pour les firmes étrangères, pour les étrangers, et de pouvoir exporter les bénéfices et le principal. C'est le début des IDE [Nda: investissements directs étrangers] (....). On a restructuré la dette, et on s'engage dans une politique de libéralisation. La preuve aussi, c'est le dahir [Nda: décret royal] qui a instauré un véritable marché financier, la bourse de Casablanca, qui date de septembre 1993. Donc ça a été concomitant. Et c'était d'abord politique. Pour moi, ça a été d'abord politique. Hassan II a négocié avec le Mouvement national, pas à pas, les étapes de l'alternance, il a négocié avec le FMI, parce qu'il avait le couteau sous la gorge, la restructuration financière marocaine, et la presse marocaine a pu vivre une nouvelle phase, celle d'une presse indépendante.» (Entretien avec le directeur d'un quotidien marocain, 2015)

Ce processus de libéralisation économique se traduit notamment par un programme de privatisation de grandes entreprises nationales. Selon Myriam Catusse, cette «vente du patrimoine public a été l'occasion pour les détenteurs du pouvoir politique (et au premier chef le roi) de distribuer de façon arbitraire et discrétionnaire une série de privilèges et de capitaux» (Catusse, 2001, p. 273). Ce discours libéral promeut les «entrepreneurs» et la transformation du capitalisme au Maroc (Catusse, 2014 [2008]). Mohamed Oubenal et Abdellatif Zeroual (Oubenal, Zeroual, 2017, p. 14) expliquent en effet que «la valorisation du mode de gestion privé » s'est "accompagnée d'une critique acerbe de l'inefficacité des administrations publiques. Les

\footnotetext{
${ }^{2}$ On peut citer la chute du bloc soviétique, le sommet franco-africain de La Baule qui symbolise la fin du soutien de la diplomatie française aux régimes dictatoriaux et qui somme le Maroc d'entretenir sa «vitrine démocratique»; la guerre du Golfe marquée au Maroc par la position favorable de Hassan II à l'intervention étrangère qui est perçue comme « un aveu de faiblesse »; la grève générale de décembre 1990 organisée par la CDT (Confédération démocratique du travail) et I'UGTM (Union générale des travailleurs du Maroc) mais aussi la sortie du livre de Gilles Perrault Notre Ami le roi (1990), qui a eu un effet retentissant.
} 
investisseurs marocains et étrangers ainsi que les journalistes économiques critiquaient, par exemple, et parmi bien d'autres choses, la corruption des agents de l'État, les retards des services des douanes, les incohérences du système judiciaire ». Comme l'analyse encore Myriam Catusse, dans la presse " "I'entrepreneur" s'impose, en tant que figure abstraite, comme le personnage providentiel des scénarios de la libéralisation et de la "mise à niveau" nécessaire dans la perspective d'une intégration à une zone de libre-échange avec I'Union européenne - en contrepoint du bourgeois "makhzénien". Les portraits qui en sont alors tracés décrivent "une bonne façon de faire des affaires" et valident ainsi une définition de la compétence économique qui rapidement va également déteindre en matière de définition de l'excellence politique» (Catusse, 2004, p. 134). Julien Duval a bien illustré à propos de la France la participation du journalisme économique à la diffusion des principes de l'économie néolibérale (Duval, 2004).

Parce que, comme on va le voir, cette reconfiguration de l'espace de la presse marocaine via la presse économique est autorisée et/ou suscitée et/ou accompagnée par le pouvoir, elle constitue un point d'entrée utile pour comprendre le champ du pouvoir au Maroc dans ses relations avec I'espace médiatique. En effet, ce travail d'enquête vient confirmer les travaux qui ont montré combien il serait vain de différencier dans l'analyse les espaces économique, politique, social et journalistique. Contrairement à ce qu'avance Béatrice Hibou dans une analyse de ces interdépendances dans le cas tunisien (Hibou, 2008), la théorie des champs élaborée par Pierre Bourdieu et ses collègues nous paraît particulièrement féconde, précisément parce qu'elle n'implique pas une séparation de ces espaces relationnels, pointant bien au contraire leur relation permanente tout en restituant leurs formes d'autonomie relative. La montée des «entrepreneurs» en politique au Maroc (Catusse, 2008), des hommes d'affaires au Parlement (Bennani-Chraïbi, 2008) à partir de la fin des années 1990 ou les logiques de la représentation patronale (Hari, Peraldi, Tozy, 2018) témoignent de ces relations permanentes entre les champs et de la multipositionnalité de très nombreux agents. L'histoire de ces deux entreprises économiques vient donc rappeler qu'elle est indissociable des processus conjoints de «libéralisation » contrôlée des champs économique, politique et journalistique, pouvant produire une doxa incarnée dans cet extrait d'un article de L'Économiste rédigé par l'actuel responsable du groupe Éco-Médias à l'occasion du $30^{\mathrm{e}}$ anniversaire du journal et rappelant la création de "l'institution ÉcoMédias» - le mot n'est bien évidemment pas neutre - en 1991. 
«Le bon moment, c'est la fin des années de plomb et l'ère des droits de I'homme, du capitalisme triomphant et de la mondialisation. Et la liberté politique, c'est une presse libre, crédible, et donc des lecteurs. La liberté économique, c'est la concurrence, et donc de la publicité. Des lecteurs et des annonceurs, voilà le fonds de commerce d'un bon journal. Et cette réussite économique va permettre d'entretenir la réussite éditoriale. Le nouvel hebdomadaire du jeudi va ainsi être le miroir de toute cette effervescence des années 90 du Maroc: les privatisations, le démantèlement tarifaire, la mise à niveau, l'organisation du GATT à Marrakech... jusqu'à la malheureuse expérience de la "campagne d'assainissement" qui va briser ce premier élan.» (Belyazid, 2021)

Cette modeste étude de cas sur deux entreprises de presse à vocation économique met donc en lumière les enjeux autour de la production de l'offre d'informations économiques et de sa diffusion au Maroc. Ils sont non seulement énormes à l'échelle nationale, mais aussi et peut-être surtout transnationale, dans la mesure où la presse économique est un des supports à travers lesquels les visions médiatiques, économiques et politiques du Maroc se construisent. Parce que son économie est très dépendante, les autorités nationales accordent en effet une grande attention à ce qui se produit sur leur pays (Marchetti, 2017) en vue d'attirer les investisseurs étrangers et les touristes.

Ces deux études de cas présentées successivement s'appuient sur une partie d'une enquête menée avec Abdelfettah Benchenna et Driss Ksikes sur l'espace des entreprises de presse au Maroc et de leurs dirigeants. La trentaine d'entretiens ${ }^{3}$ réalisés entre 2015 et 2017 et les recherches documentaires sur lesquelles repose ce travail portent sur un échantillon représentatif des différents pôles contemporains de cet univers de la presse papier et électronique marocaine privée. Ces interviews ont été menées essentiellement avec des fractions dominantes du champ journalistique marocain, c'est-à-dire des patrons des principaux supports de la presse papier et électronique ainsi qu'avec des journalistes ayant travaillé dans plusieurs titres structurant ou ayant structuré fortement l'espace journalistique depuis les années 1980. Pour compléter, nous avons effectué un travail documentaire à partir d'ouvrages et d'une revue de presse, mais aussi collecté des données disponibles sur les dirigeants sur le site charika.

\footnotetext{
${ }^{3}$ Pour d'autres aspects de cette enquête, je me permets d'indiquer les références suivantes: Benchenna, Ksikes, Marchetti, 2017; Benchenna, Marchetti, 2019 et 2020.
} 
$\mathrm{ma}^{4}$. Ces données des tribunaux de commerce ont également permis de saisir I'histoire économique et juridique des entreprises de presse.

\section{Un groupe au sein du pouvoir économique et politique}

Les instigateurs les plus visibles des sociétés de la presse économique marocaine ont créé celles-ci à un moment où il s'agissait, comme on l'a déjà mentionné, d'accompagner le développement économique et financier du pays à la fin des années 1980 et au début des années 1990. Cette presse se veut le symbole de la "modernité», de l'«ouverture» économique et politique, des «forces progressistes». Ce n'est d'ailleurs pas un hasard si Jean-Louis Servan-Schreiber (souvent appelé par ses initiales JLSS), un des pionniers de la presse économique en France avec la création du magazine L'Expansion et la participation dans plusieurs titres économiques, investit en 1991 puis reprend en 1994 et pour trois ans I'hebdomadaire La Vie économique ${ }^{5}$, notamment via sa femme, de nationalité marocaine, Perla Servan-Schreiber, qui s'occupa de la publicité et du marketing. Il décrit ainsi cette opportunité qui fait suite à la cession de parts de son groupe la même année:

«Le hasard a fait que j'étais à la tête d'un groupe de presse qui s'appelle le groupe Expansion que j'avais créé, développé, qui était autour du magazine $L^{\prime}$ Expansion, et ce magazine avait fait une... disons une expansion internationale dans des pays d'Europe. On a acheté à ce moment-là (...) environ dans une dizaine de pays d'Europe des titres de différentes sortes, mais toujours en rapport avec l'économie, et un jour quelqu'un m'a dit: il y a Marcel Herzog qui veut vendre La Vie éco, si ça vous intéresse, etc. (Entretien avec Abdelfettah Benchenna, Driss Ksikes et I'auteur, 2017)

Plusieurs enquêtés mentionnent, comme certains journalistes publiquement (Islah, 2020), qu'il était «à l'époque (...) considéré comme étant proche du conseiller royal André Azoulay mais aussi de Fouad Filali, ex-gendre de Hassan II et ex-PDG de la holding royale ONA» (Le Desk, 2020). Selon Annemarie Iddins (Iddins, 2020, p. 3), autrice d'un article sur ce passage de JLSS à La Vie économique, «les médias deviennent un mécanisme pour produire un discours sur l'exceptionnalisme marocain et la transition vers la démocratie».

\footnotetext{
${ }^{4}$ L'accès à ces données payantes a été possible grâce à un financement de l'ERC Tarica.

${ }^{5}$ On trouvera des détails très précis de cette expérience dans cet article parfois hagiographique et écrit en «hommage à Jean-Louis Servan-Schreiber »: Iddins, 2020.
} 


\section{Les conditions d'une alliance}

Deux autres couples incarnent de manière beaucoup plus concrète et durable cette émergence de la presse économique au croisement des champs politique, économique et journalistique, en vue de promouvoir cette «libéralisation » politique et économique à travers un projet journalistique: d'un côté, Marie-Thérèse Bourrut, plus connue sous le nom de Nadia Salah, et Abdelmounaim Dilami à la tête d'Éco-Médias entre 1991 et 2020; de l'autre, Afifa Dassouli et Fahd Yata à la direction d'Impression Presse Édition depuis 1995. Tous nés dans les années 1950, ils vont mettre en place deux entreprises de presse stricto sensu et en assurer progressivement la pérennité, comme le montre la place des deux sociétés dans l'espace de la presse au Maroc en 2021. Au moment où ils lancent leurs titres, il s'agit de s'adresser à un public urbain, diplômé et fortement doté en capital économique, essentiellement francophone à l'époque, qui est d'ailleurs régulièrement consulté: "Tous les deux ans on fait faire une étude (...) ça permet de recadrer en cas de besoin », explique Abdelmounaim Dilami (entretien, 2015).

Cette dimension fortement commerciale du projet doit probablement beaucoup à Marie-Thérèse Bourrut, son épouse, qui a réalisé des études à dominante économique. Ancienne élève de I'Institut d'études politiques de Grenoble (1974-1977), option économie financière, elle est titulaire de plusieurs diplômes universitaires de science économique. Elle acquiert ses premières expériences dans le journalisme en France, étant en charge des questions d'énergie et européennes à la Société générale de presse (SGP), qui édite des supports à diffusion restreinte sur abonnement. Elle travaille aussi et surtout très rapidement au Maroc où elle s'installe avec son conjoint à la fin des années 1970. Elle exerce comme la plupart des journalistes de la presse écrite de l'époque dans un quotidien partisan, celui du parti conservateur de I'Istiqlal, L'Opinion, en 1978-1979, puis entre 1981 et 1989 où elle couvre les questions économiques. Elle est correspondante au Maroc de l'hebdomadaire français Le Moniteur des travaux publics (1981-1992) et du magazine économique Middle East Economic Digest (1983-1994).

Outre sa contribution à un journalisme économique encore naissant, Marie-Thérèse Bourrut participe de l'intérieur au processus de mise en œuvre du programme d'«ajustement structurel» faisant suite aux injonctions du FMI. En effet, elle est à la fois consultante pour le ministère des Travaux publics (1984-1991) et surtout conseillère (1986-1991) au cabinet de Mohamed Berrada, ministre de l'Économie et des Finances. 
C'est après avoir effectué un passage de plus de deux ans à la rédaction en chef de La Vie économique (1989-1991), le principal journal économique marocain de l'époque, qu'elle va contribuer à moderniser (entretien avec un de ses confrères, 2015), que Marie-Thérèse Bourrut fonde en 1991 avec son mari la société Éco-Médias (Park et Boum, 2016, p. 540-541; Sabib, 2012). Alors professeur de droit à l'université Mohammed V de Rabat, Abdelmounaim Dilami explique qu'ils ont saisi ces «opportunités politiques et économiques» (entretien, 2015). Né à Fès en 1950, ce docteur d'État en droit public et science politique de I'Université de Grenoble explique qu'il s'est s'engagé dans la presse "par le mariage», tout en continuant en parallèle sa carrière universitaire.

"Il se trouve que mon épouse, quand on est rentré au Maroc, avait commencé à travailler dans la presse économique. Donc, lorsque ces opportunités politiques et économiques sont apparues, on s'est dit: "bon, on va se lancer et on va créer une entreprise effectivement", pour accompagner tout ce processus dont je vous ai parlé. Donc comme quelqu'un l'avait dit une fois, je suis venu un petit peu par alliance, par le mariage.» (Entretien, octobre 2015)

Le couple Dilami-Bourrut s'est associé dès le départ à Khalid Belyazid, qui a fréquenté le lycée mixte de Fès avant d'effectuer également ses études en France (doctorat de gestion à Nice), puis d'intégrer la direction des ressources humaines de deux entreprises (de médicaments et de jean's) (L'Économiste, 2016). Membre du Conseil d'administration et doté de quelques parts dans la société, il va au fil des années monter dans la hiérarchie du groupe, en étant respectivement secrétaire général de la rédaction, directeur du développement à partir de 1997 (L'Économiste, 1997), directeur général depuis 2001. Parmi les cofondateurs de L'Économiste figure aussi Nader Mawlawi, un Libanais qui a également fait ses études (notamment en démographie) en France et dirige en 1991 le groupe Sunergia, spécialisé dans les études de marché, «qui fit l'étude de marché pour le lancement» du journal (Belyazid, 2021). C'est sa société qui réalise de nombreuses études publiées dans le journal. C'est donc le contexte de libéralisation politique et économique qui est propice au lancement de titres économiques.

"C'était en 91 quand on a démarré, et c'était l'ouverture de la libéralisation économique du pays, et la libéralisation économique allait amener deux choses. Bon, peu à peu, les choses allaient se rationaliser, par la force des choses, parce que, vu les accords, les échanges du Maroc avec l'Europe, on est bien obligé de commencer à y ressembler un peu... Et puis la libéralisation économique, qui était une nécessité à l'époque, c'était pas... juste un choix 
comme ça allait forcément entraîner peu après une libéralisation politique. Donc un marché de l'information allait devenir important, la disponibilité de l'information, sa fluidité allaient devenir des choses très importantes pour la société. Donc on avait misé sur ça.» (Entretien avec Abdelmounaim Dilami, 2015)

Non seulement le lancement d'un titre économique, dont la parution était hebdomadaire en 1991, accompagne par son offre journalistique ce processus de «libéralisation » économique, mais celui-ci est aussi et surtout suscité et/ou accompagné par certaines fractions du pouvoir, très probablement André Azoulay, conseiller du Roi Hassan II ${ }^{6}$, et Mohamed Berrada, avec lequel a travaillé Marie-Thérèse Bourrut quand il était ministre des Finances.

«Moi je pense que c'est l'influence de personnes comme André Azoulay, qui était conseiller économique du roi... (...) Je pense que tout ce qui est presse économique, etc., c'est Nadia Salah et Dilami qui ont fait leur journal sous... comment dire... avec le soutien, mais aussi comment dire... la... [il cherche ses mots] pas la pression... pas la pression mais les encouragements évidents de... André Azoulay et de Si Mohammed Berrada, qui était à l'époque ambassadeur à Paris.» (Entretien avec un cadre dirigeant de la presse économique, 2017)

De ce fait, les grandes entreprises nationales vont tout d'abord acheter de la publicité, qui constitue la principale source de revenus du titre: au premier semestre 2005, L'Économiste, qui vend alors environ 10000 exemplaires, «s'adjuge à lui seul près de $15 \%$ des investissements publicitaires dans la presse écrite, alors que ses ventes ne représentent que $3,33 \%$ du total marocain ! II y a là un mystère commercial qui mérite une étude plus approfondie...», fait remarquer ironiquement le journaliste Robert Assaraf dans Jeune Afrique (Assaraf, 2005). Il en va de même pour son confrère La Nouvelle Tribune lancé en 1995 par Fahd Yata et Afifa Dassouli: «Malgré les quelques faibles milliers d'exemplaires annoncés vendus,

\footnotetext{
${ }^{6}$ Né en 1941, diplômé du Centre de formation des journalistes (CFJ) à Paris, André Azoulay a lui-même commencé une carrière de journaliste professionnel dans les années 1960 étant tout d'abord rédacteur en chef du quotidien Maroc Informations et correspondant d'Europe 1 pour l'Afrique du Nord à Casablanca entre 1963 et 1966 avant de partir en France où il a été journaliste au service étranger du Monde (1966-1968). Après un passage de deux ans comme directeur du service des études de la holding royale I'ONA, il commence une carrière dans une des plus grosses banques françaises à Paris: il est directeur du groupe SCOA Paris (Société commerciale de l'Ouest africain) appartenant à cet établissement pendant les années 1970, puis directeur de la communication de Paribas (1980-1990). Il est nommé conseiller du roi Hassan II en novembre 1991 (biographie de la presse, 2017).
} 
le journal recueille 6,3 millions de dirhams, soit, pour certains numéros, proportionnellement quatre fois plus que La Vie économique et six fois plus que TelQuel.» (Ibid., 2005)

Au-delà de la publicité, certains grands groupes vont surtout investir directement dans Éco-Médias: "On a fait un tour de table avec les institutionnels, c'est-à-dire que Nadia Salah et moi, nous avons $60 \%$ du capital, et puis nous avons avec nous des institutionnels qui ont en 10 chacun. Donc c'est un tour de table assez large, mais dont nous détenons quand même la majorité », explique Abdelmounaim Dilami (entretien, 2015). En effet, la société anonyme était constituée au moment de notre enquête à plus de $60 \%$ par le couple et le reste par des partenaires historiques, des investisseurs institutionnels liés aux fractions dominantes du champ économique et politique, c'est-à-dire très largement francophones.

Parmi eux, dès le départ, figurent les grands groupes marocains. Audelà de l'Omnium nord-africain (ONA), propriété de la famille royale, on trouve de grandes entreprises du pays, proches du pouvoir royal : la Société marocaine de participation (SOPAR), holding familial d'une des plus grandes fortunes du pays de l'époque, Moulay Ali Kettani, issu d'une grande famille fassie nationaliste et qui a été à la tête de la Chambre de commerce et d'industrie de Casablanca (Hachim, 2006, p. 209-210) et surtout de Wafabank (Kaioua, 1993, p. 328-329). Il sera remplacé par son fils aîné, Saâd, un ancien diplômé de l'École supérieure de commerce de Paris (ESCP) marié à la fille d'Abdelhadi Boutaleb, conseiller royal sous Hassan II, qui vendra la holding familiale à la BCM en 2003, pour former le groupe Attijariwafa Bank, filiale de la holding royale la Société nationale d'investissement (SNI).

S'ajoute l'un des principaux banquiers marocains de l'époque, Abdelaziz Alami, qui représente la Banque commerciale du Maroc (aujourd'hui Attijariwafa Bank), dans laquelle la holding royale était actionnaire et qu'il dirige au milieu des années 1960. La BCM a pris $15 \%$ du capital de départ du journal (1 million de dirhams) avant, plus tard, de céder ses parts aux deux fondateurs (M.A.B., 2014). S'ajoute enfin comme investisseur institutionnel le groupe KAT spécialisé dans l'agro-alimentaire, détenu également par une grande famille fassie, les Ouazzani, notamment Thami Ouazzani Touhamy. Cet avocat formé à Paris a été nommé en 1956 ministre de la Production industrielle et des Mines, pour ensuite occuper des postes d'ambassadeur du Royaume, de ministre du Travail et des Affaires sociales, du Tourisme et se lancer avec succès dans les affaires dans les années 1970 (Douyeb, 2003). 
Dans le numéro anniversaire des 30 ans de L'Économiste, Khalid Belyazid (Belyazid, 2021), son directeur général, mentionne également la participation de deux hommes d'affaires très liés dans leur parcours et nés au début des années 1960. Le premier, Aziz Akhannouch, revient au Maroc en 1990 après un MBA à l'université de Sherbrooke au Québec pour intégrer l'une des filiales du groupe de son père, spécialisé dans les énergies et le pétrole (Berraoui, 2006; Boum, Park, 2016, p. 33-34; Ahougnon, 2021). II deviendra au décès de celui-ci l'actionnaire majoritaire d'Akwa, qui va investir dans Éco-Médias. Le second, Moulay Hafid Elalamy, est également diplômé de l'université de Sherbrooke au Canada (Duteil, 2014) où il a obtenu un diplôme en ingénierie informatique. Après un bref passage au cabinet du ministère des Finances au Canada et au sein d'un groupe québécois, il rentre lui aussi dans son pays natal (à la fin des années 1980) pour travailler directement - il en sera notamment le secrétaire général à partir de 1994 - puis indirectement pour la holding royale ONA dans le secteur des assurances (Tovi, 2007) au moment de la création d'Éco-Médias.

L'élargissement du conseil d'administration du groupe en 1999, afin de se conformer au nouveau texte sur les sociétés anonymes (L'Économiste, 1999), montre combien ses cofondateurs s'entourent d'autres grands noms qui incarnent les fractions dominantes du champ du pouvoir marocain à l'époque et ont participé très directement au processus de financiarisation et de privatisation de l'économie marocaine mené sous le contrôle des conseillers du Palais royal. En effet, quand il entre au conseil d'administration d'Éco-Médias, Driss Jettou a déjà été plusieurs fois ministre entre 1993 et 1998, notamment en charge du Commerce et de I'Industrie et des Finances, c'est-à-dire un des artisans de la mise en œuvre du programme de privatisation. "À la mort de Hassan II (en 1999), Jettou est chargé de mettre de l'ordre dans les affaires privées du souverain défunt. II prend la tête de Siger (anagramme de Regis), actionnaire de référence de la holding royale ONA», explique ainsi le journaliste Jules Crétois (Crétois, 2018). II sera notamment PDG de I'Office chérifien des phosphates en août 2001, puis Premier ministre (2002-2007). Fils d'un épicier d'El Jadida, il incarne l'ascension sociale par les affaires industrielles. Employé chez la marque de chaussures Bata, il se forme au management et à la gestion d'entreprise au Cordwainers College à Londres avant de fonder sa propre marque concurrente, Au Derby, devenant également président de la Fédération du textile et du cuir à la CGEM et vice-président de I'Association marocaine des exportateurs (ASMEX). Il devient ensuite un "grand commis de l'État» souvent décrit comme proche de I'USFP. 
Deux autres nouveaux entrants au conseil d'administration sont des ingénieurs formés en France, dont les carrières sont plus directement liées à l'État. D'un côté, Driss Benhima est issu «d'une famille de grands commis de l'État originaire de la ville de Safi», fils de l'ancien Premier ministre Muhammad Benhima (1966-1969) «qui a été le premier président marocain de I'ONA en 1969 » (Catusse, 2014 [2008], p. 112). Son parcours est également très fortement lié au champ bureaucratique. Diplômé de I'École Polytechnique de Paris et de l'École nationale supérieure des Mines à Paris, il a assuré différentes charges à l'OCP, qui est encore une entreprise publique, entre 1978 et 1990, avant de rejoindre le secteur privé, devenant administrateur d'une branche du groupe Air Liquide. Comme d'autres membres du conseil d'administration d'Éco-Médias, il en est membre après avoir été, à la fin du règne de Hassan II (1997-1998), ministre des Transports, de la Marine marchande, du Tourisme, de l'Énergie et des Mines, et avoir présidé l'Office national d'électricité (ONE) à partir de 1994. D'un autre côté, Abdelazziz Meziane-Belfikh, ingénieur ancien élève des Ponts-et-Chaussées, a travaillé à différents postes de responsabilité pour le ministère des Travaux publics à partir de 1968, est devenu notamment secrétaire général des Travaux publics à partir de 1983 et a été nommé au conseil d'administration en 1999 après un mandat de ministre de l'Agriculture (1997-1998). Il est alors conseiller de Mohammed VI après avoir occupé la même fonction auprès de son père.

Enfin, d'autres profils de grands dirigeants, qui ont tous fait leurs études en France, renvoient davantage au pôle financier de l'espace économique marocain. C'est le cas d'Adil Douiri qui, après l'obtention de son diplôme de l'École nationale des Ponts-et-Chaussées en 1985, débute à BNP Paribas jusqu'au début des années 1990 avant de cofonder en 1992 un grand groupe financier, le Casablanca Finance Group (CFG), chargé de développer les marchés financiers au Maroc. Son associé, Amyn Alami, qui rejoint également le conseil d'administration d'Éco-Médias, est un spécialiste de la finance, ancien diplômé de I'IEP Paris, qui commence également à travailler dans un groupe bancaire français, au département de recherche et d'arbitrage de la Compagnie financière Edmond de Rothshild-Banque. Quand il entre au conseil d'administration en 1999, il a été entre 1995 et 1998 président délégué de la Bourse de Casablanca ouverte en 1993.

La création de la société Écoprint en 1997, qui gère l'imprimerie du groupe Éco-Médias, témoigne également de ce fort ancrage du groupe au sein du champ du pouvoir. Les deux autres principaux membres d'Écoprint indiqués par le greffe (Charika, 2020) incarnent aussi les fractions 
dominantes de cet univers. En effet, Moulay Hafid El Alamy y représente désormais son groupe Saham. On retrouve Abderrahmane Saaidi également formé aux affaires en France (licence en droit des affaires à Montpellier, diplôme de l'École supérieure de commerce de Montpellier en 1973 et diplôme d'expert-comptable en 1976). II a notamment enseigné à I'Institut supérieur du commerce et d'administration des entreprises (ISCAE) de Casablanca. En 1985, il fonde Saaidi \& Hdid Consultants, I'un des premiers cabinets marocains de conseil et d'assistance en matière de droit, fiscalité, comptabilité et finance d'entreprise. Ce fils d'ébéniste est surtout l'un des principaux artisans des privatisations et des réformes fiscales puisqu'il a été ministre de l'Incitation de l'économie, de la Privatisation, délégué auprès du Premier ministre, chargé des Établissements de l'État en 1995 et surtout ministre de la Privatisation entre 1997 et 1998, avant d'être nommé en 2001 directeur général de la SAMIR, la raffinerie du Maroc, qu'il avait lui-même privatisée (Manyani, 2020; Maroc Hebdo, 2020).

Abdelmounaïm Dilami s'appuie également sur des représentants de la France au Maroc dans l'imprimerie comme dans la communication. En effet, il s'allie quasiment à parité dans Écoprint avec l'homme d'affaires français Jean-Luc Martinet, nommé directeur délégué, qui est un des principaux représentants français du commerce et de l'industrie au Maroc. Il est alors à la tête depuis 1985 de sa première entreprise, EXIPP, spécialisée en courtage international de papiers cartons. Ce Français du Maroc est issu d'une riche famille chrétienne de promoteurs de Casablanca, un quartier de la ville portant le nom de Martinet en hommage à son grand-père Odil (Derb Lihoudi aujourd'hui). Son oncle, Guy Martinet, historien et archéologue, a lutté pour l'indépendance du pays, et le mari de sa mère, Charles Gonard, un célèbre résistant, compagnon de la Libération (Hopquin, 2016), qui a contribué à l'élever, était connu pour ses positions anticoloniales. II a travaillé vingt-huit ans au Maroc, dirigeant d'ailleurs une ancienne entreprise coloniale, la Compagnie chérifienne des textiles (CCT). Après des études d'économie à l'université Mohammed V de Rabat et de gestion à l'université de Montpellier, Jean-Luc Martinet a commencé sa carrière à la direction financière d'une société de leasing au Maroc avant d'être directeur général de la société Le Carton (1977-1984) et de se lancer dans l'entrepreneuriat Dans les années 2000, il présidera la Chambre française de commerce et d'industrie du Maroc, sera représentant de I'UMP au Maroc (2009-2013) et

\footnotetext{
${ }^{7}$ Source principale: http://www.aixor.com/pages.php?pid=3
} 
surtout créera en 2001 la holding financière Aixor qu'il dirige et dont il est le représentant au sein d'Éco-Médias et d'Écoprint.

De même, quand il fonde Éco Studies en 2008, chargé de gérer l'École supérieure de journalisme et de communication (ESJC) qui a été créée en plein centre de Casablanca pour lutter contre la pénurie de journalistes francophones, Abdelmounaïm Dilami enrôle deux spécialistes français de la communication comme administrateurs: Cyrille Benoist, président et principal actionnaire de la Société par Actions Simplifiée (SAS) Learning Management Development (LMD), dont le capital est de 3,9 millions d'euros en $2007^{8}$, qui va présider le conseil d'administration et Guillaume Jobin son associé. Diplômé de l'Institut supérieur de gestion (ISG) en 1986, le premier se spécialise dans le conseil en communication (Biographie de la presse, 2017); il est le fils d'un conseiller-maître à la Cour des comptes qui a travaillé dans de nombreux cabinets ministériels entre les années 1950 et 1970, présidant à sa retraite le Secours catholique (1995-1997). Cyrille Benoist commence (1981-1988) à la direction générale de l'information du Conseil national du patronat français (CNPF) et fonde dans les années 1990 et 2000 plusieurs sociétés dans le conseil en communication (BIA, Décision Communication), la veille plurimédia (Presse+). Il se lance au début des années 2000 dans l'enseignement privé sur cette matière en présidant le Groupe ECS (European Communication School) entre 2003 et 2008. Il créé en 2003 Learning Management Development (LMD) en développant, surtout à partir de 2006, I'École supérieure de journalisme (ESJ), une école privée basée à Paris fondée en 1896, qu'il a rachetée. II va la présider entre 2006 et 2008 avant de céder sa place à son associé Guillaume Jobin qui la préside encore en 2021.

C'est surtout ce dernier qui s'installe en partie au Maroc à partir de 2008 et va développer les liens entre les deux écoles. Docteur en médecine et titulaire d'un Master of Business Administration (MBA) de l'École des hautes études commerciales (HEC), il est issu d'une grande famille de négociants en café au port du Havre, la Maison Paul Jobin \& compagnie fondée à la fin du XIX siècle, qui a développé le commerce avec les colonies françaises. Son père, Philippe Jobin, a fait sa carrière au sein de l'entreprise familiale - sa mère y est également fortement impliquée - qu'il reprend au début des années 1970 pour la vendre en 1988, ses héritiers ne souhaitant pas poursuivre (Hanachi, 2015, p. 290-294). Après ses études supérieures,

\footnotetext{
${ }^{8}$ Les données juridiques et financières sur les sociétés françaises sont extraites de la base Cap Financials, et les données biographiques sont issues des fiches des intéressés constituées par lesbiographes.com et de leurs pages Linkedln.
} 
Guillaume Jobin a d'abord travaillé pendant vingt ans en France dans le marketing médical et la finance (1986-2006), publiant un ouvrage Exporter et investir en Russie paru aux éditions Vuibert en 1995. II se lance ensuite dans l'enseignement privé spécialisé dans la communication et le journalisme au milieu des années 2000. Il le fait via LMD et surtout en reprenant seul en 2008 I'ESJ Paris (ESJP) qu'il va développer à l'international via sa société ESJ Développement. Au-delà de son association avec Éco Studies - Abdelmounaïm Dilami est également associé dans ESJ Développement -, il s'alliera au Maroc à l'École de management et des sciences informatiques (EMPSI) et ouvrira une antenne à Rabat avec I'Institute for Leadership and Communication Studies (ILCS) (Maghreb confidentiel, 2015). II a développé également plus tard des formations en Algérie, en Tunisie et au Sénégal.

\section{La préservation d'un capitalisme familial}

Pour autant, ce fort ancrage dans le champ du pouvoir au Maroc et ses alliances avec des Français vivant au Maroc ne doivent pas faire oublier la dimension familiale du groupe Éco-Médias ${ }^{9}$, qui cherche à préserver une forme d'autonomie en gardant le contrôle à travers un actionnariat majoritaire. C'est ce qui a permis aux fondateurs de résister (visiblement à plusieurs reprises) à des tentatives de rachat, notamment de la part du groupe Caractères d'Aziz Akhannouch. À la fin de la décennie 1990, Nadia Salah expliquait ainsi dans un entretien à Gilles Kraemer que, "en 1997, certains de nos actionnaires ont acheté La Vie économique à M. ServanSchreiber, ce qui a posé un problème de concurrence déloyale puisque les deux titres sont presque sur le même créneau. Nous avons attaqué ces deux actionnaires qui voulaient mettre en place un empire médiatique contre nous» (Kraemer, 2001, p. 151-152). De même, Négib Bouderbala explique au début des années 2000 que «ses statuts lui ont permis de résister à trois tentatives de prises de contrôle du journal par des groupes extérieurs» (Bouderbala, 2001, p. 34-35). Ce fut aussi visiblement le cas en 2019, selon la lettre Maghreb confidentiel (2019), qui fait état d'une nouvelle tentative du groupe Caractères avant la vente d'Éco-Médias à la holding Trispolis, détenue par Best Financière, un leader de la distribution avec notamment Label Vie, Carrefour, et par le groupe Sunergia, actionnaire historique.

\footnotetext{
${ }^{9}$ Cette dimension quasi systématique dans les entreprises de presse marocaine, et plus généralement dans de nombreuses entreprises, ne peut être traitée ici et fera l'objet de développements ultérieurs.
} 


\section{Le mariage de l'économie, de la politique et de la presse}

Un autre couple de la même génération et semblable sous de nombreux aspects s'est engagé dans la presse économique, en léger décalage avec Marie-Thérèse Bourrut et Abdelmounaim Dilami. C'est en effet le 30 novembre 1995 que la société anonyme Impression presse édition (IPE), qui édite I'hebdomadaire La Nouvelle Tribune, créée par Fahd Yata et son épouse Afifa Dassouli, est immatriculée. Originaire selon son expression de la «bourgeoisie fassie» istiqlalienne, Afifa Dassouli a rencontré dans le courant des années 1970 son futur mari à I'Union nationale des étudiants marocains (UNEM) à Paris. Si son père était un militant du parti conservateur nationaliste, elle se décrit alors comme une "sympathisante de I'USFP " (Oiknine, Mrani, 2011, p. 62). Fahd Yata est, lui, membre d'un autre parti de gauche, le Parti du progrès et du socialisme (PPS), dirigé par son père qui anime également son organe de presse, le quotidien Al Bayane. Ali Yata, grande figure de la vie politique marocaine, avait été secrétaire général du Parti communiste marocain (PCM) à partir de 1945, parti qui a été banni entre 1950 et 1968, puis de sa «réincarnation», le PLS (Parti de la libération et du socialisme) à la fin des années 1960. Il a été emprisonné sous la colonisation française, et il est en exil en région parisienne quand son fils Fahd Yata naît en 1952. II revient dans le Maroc indépendant à la fin des années 1950, obtenant la nationalité marocaine par décision de Mohammed V et de Hassan II, alors prince héritier, pour son engagement dans la lutte pour le recouvrement de la souveraineté du pays.

Fahd Yata est donc élevé au sein de la gauche du champ politique marocain où il acquiert, via son père notamment, non seulement un capital politique et un capital social, mais aussi un double capital professionnel dans le journalisme et à l'université. En effet, socialisé dans un univers francophone comme Abdelmounaim Dilami, il réalise des études longues au Maroc et en France. Élève du lycée Lyautey de Casablanca, I'un des établissements français les plus prestigieux du pays, il poursuit des études de droit et de science politique en France, obtenant notamment un DEA et un doctorat en relations internationales à l'Université Paris I PanthéonSorbonne (1978-1979). S'il occupe rapidement à son retour au Maroc un poste d'enseignant-chercheur à la faculté de droit de I'Université Hassan II de Casablanca, il investit en même temps l'espace journalistique marocain dans lequel il a été formé très tôt, comme son frère Nadir, également journaliste, par son père et dans «son » journal Al Bayane où il a été rédacteur 
entre 1979 et 1986 (entretien, 2017). Ce sera également le cas d'une autre figure de la presse écrite marocaine, Aboubakr Jamaï, le fils de Khalid Jamaï, journaliste de L'Opinion, le quotidien du parti de l'Istiqlal. Fahd Yata continue à se former à l'encadrement et au fonctionnement des entreprises de presse en devenant successivement directeur de rédaction du magazine Tijaris, édité par le Centre islamique pour le développement du commerce (CIDC) à Casablanca (1986-1988), du magazine Enjeux (1989-1991), avant de rejoindre à moins de 40 ans la direction de l'information et des magazines de la chaîne privée $2 M$ (1991-1992).

«Un jour, je ne sais pas, c'était fin 90 ou 91, à un feu rouge, il y a un grand $4 \times 4$ noir, la vitre s'abaisse, et il y a Fouad Filali et Driss Basri qui me dit: "Monsieur Yata? Est-ce que vous êtes libre à déjeuner demain ?" Je dis: "Oui, bien sûr." "Venez nous rejoindre demain." Et là, il me dit: "Monsieur Yata, voilà, j'aimerais que vous preniez la direction de l'information de 2M." Pour moi, c'était une projection dans la stratosphère, parce que je connaissais $2 M$, c'était une télé qui venait à peine de naître. Bon elle était câblée, cryptée, payante, mais Fouad Filali était au faîte de sa puissance à ce moment-là, le gendre du roi. À I'ONA, il prenait de l'importance, etc. dans les affaires. (...) Et donc, et c'était 90-91, on sentait que ça bougeait et ça bougeait parce que... c'est à ce moment-là que la maladie du roi a été révélée. Il avait été hospitalisé aux États-Unis, etc. (...) et lui a compris, le roi a compris lui-même qu'il devait préparer sa succession, son départ. Basri a compris qu'il avait quelques instruments pour faire du fric, donc il s'est mis à fond dans les affaires, mais aussi ça a mené vers l'amnistie des détenus, des exilés en 94. $2 M \ldots 2 M$ était un espace de liberté. (...) Et je pense que $2 M \ldots$ a fait le lit de la presse indépendante marocaine. Ou de la presse commerciale marocaine (...) Parce que $2 M$ est sortie en 89 , L'Économiste, il a commencé (...) en $91 \ldots$ Maroc Hebdo aussi, 91.» (Entretien, 2017)

C'est après avoir été rédacteur en chef de La Vie économique (1992-1995) qu'il lance La Nouvelle Tribune. Le projet de titre économique du couple est visiblement «inspiré par la démarche et les conseils» de ses «amis de L'Économiste», expliqua rétrospectivement Fahd Yata (L'Économiste, 1996). Le projet, inspiré de La Tribune en France, est en lien direct avec une volonté politique: "En fait, le plus gros holding marocain, l'Omnium nord-africain (ONA) cherchait à créer un titre économique», raconte-t-il dans un entretien à la fin des années 1990 (Kraemer, 2001, p. 151).

Son épouse, Afifa Dassouli, a joué un rôle capital dans tous les sens du terme, comme Marie-Thérèse Bourrut dans le cas du groupe Éco-Médias. À l'instar de sa consœur, elle dispose d'une solide formation en économie financière acquise en France et sera également une des pionnières dans l'émergence de ce journalisme spécialisé. En effet, titulaire d'une maîtrise 
de gestion, d'un DESS de gestion financière de l'université de Paris I et d'un doctorat, elle a non seulement apporté son savoir-faire dans la production de contenus spécialisés dans le domaine financier, réalisant le «premier cahier "saumon" du Maroc» (Le Desk-Reporters sans frontières, 2021), mais aussi dans la gestion même de la société dont elle est toujours directrice administrative et financière. Il est révélateur que, à l'occasion du quinzième anniversaire de la naissance de La Nouvelle Tribune, Fahd Yata I'ait remerciée publiquement en précisant que c'est à elle que «revient le mérite d'avoir conçu et géré La Nouvelle Tribune comme doit l'être une entreprise » (L'Économiste, 2011). Ils racontent ainsi la naissance de leur hebdomadaire.

«Et moi, en pur produit de ma formation, je lui ai suggéré de créer son propre journal. Je lui ai proposé de monter un business-plan et d'aller chercher des investisseurs: s'ils nous font confiance tant mieux; si ce n'est pas le cas, nous ferons marche arrière. II n'y croyait pas: j'ai donc fait le business plan, j'ai commandé une étude de marché, etc. Et il s'est avéré que les investisseurs étaient enthousiastes.» (Afifa Dassouli citée par Oiknine, Mrani, 2011, p. 62)

«À l'époque, c'était en 95, j'avais 43 ans. Elle m'a dit: “Fahd, tu vas continuer à travailler pour les autres? - Oui, pourquoi? Je ne devrais pas avoir de problème pour trouver un job quelque part. - Non, je crois que c'est maintenant ou jamais, ou tu fais ton journal, ou tu ne seras jamais indépendant. - Mais Afifa, l'argent... - L'argent? Je pense que tu as assez de notoriété pour en trouver." » (Entretien avec Fahd Yata, 2017)

Comme Marie-Thérèse Bourrut, cette enseignante de I'ISCAE a été impliquée dans le processus de privatisation et de financiarisation de l'économie marocaine, nouant des relations avec les principaux agents des fractions dominantes du champ du pouvoir, qui seront mobilisés au moment de la création d'IPE. En effet, comme elle le raconte, dans le courant de son PhD au Canada au début des années 1990, Afifa Dassouli fait un stage dans la célèbre firme de courtage Salomon Brothers au Maroc, qui crée alors un fonds qu'elle va "gérer»: "C'est ce que j'ai fait pendant quatre ans sous I'autorité de grandes personnalités marocaines comme MM. Mohamed Berrada et Fouad Filali.» (Oiknine, Mrani, 2001, p. 62) Elle participe ainsi au fonctionnement du Morocco Fund lancé à cette époque par Salomon Brothers et la holding royale ONA pour «mobiliser en faveur du Maroc des capitaux étrangers (...), promouvoir les investissements et la création d'emplois, (favoriser) l'émergence du marché financier et l'ouverture du capital des entreprises marocaines» selon les termes de son communiqué (Salah, 1994). Mohamed Berrada, qui devient président de la société de gestion du Morocco Fund, a été ministre des Finances de 1986 à 1993, c'està-dire un des principaux animateurs du processus de réformes touchant le 
commerce extérieur, la monnaie, les réformes fiscales et la transformation des marchés financiers. Quant à Fouad Filali, il est le fils d'Abdellatif Filali, ancien ministre à la fin des années 1960 et durant les années 1970, diplomate et Premier ministre (1995-1998), issu d'une grande dynastie marocaine. Fouad Filali est alors le président du conseil d'administration du Fonds et à la tête de la holding royale, étant encore également le mari de Lalla Meryem, la fille aînée de Hassan II.

Fahd Yata, le mari d'Afifa Dassouli, explique très franchement en entretien le rôle direct de son épouse non seulement dans la décision de lancer le journal, mais aussi dans la constitution du tour de table de la société, tout particulièrement quand il a fallu faire appel à deux des plus importants banquiers marocains à l'époque: Abdelaziz El Alami, qui était également un des actionnaires de L'Économiste dès 1991, était alors président de la Banque commerciale du Maroc (BCM) dans laquelle I'ONA avait des parts; Othman Benjelloun, qui était devenu en juin 1995 le président de Banque marocaine du commerce extérieur (BMCE), banque privatisée cette annéelà et dont il a pris le contrôle par le biais d'une des filiales d'un important groupe familial, la Royale marocaine d'assurance (RMA) (Catusse, 2008, § 49). Cette année-là, il fonde également la Chambre américaine du commerce au Maroc, et il est désigné président du Groupement professionnel des banques marocaines (GPBM).

«À travers les levées de fonds du Morocco Fund, elle avait eu accès à tous les grands patrons économiques du Maroc. Donc j'ai pris rendez-vous avec deux hommes: Abdelaziz El Alami, qui était le PDG de la Banque commerciale du Maroc, un esprit brillantissime, un intellectuel de valeur qui citait Proust dans le texte $A$ la recherche du temps perdu qu'il connaissait par cœur, entre autres, mais d'une grande, grande culture. Et c'était des gens qui avaient des idées bien arrêtées, qui étaient de grands capitalistes, mais il y avait un côté humaniste et surtout beaucoup de respect pour le Mouvement national et pour le Parti communiste. Je l'ai senti, parce qu'ils m'ont aidé. Donc Abdelaziz El Alami et Othman Benjelloun, le patron de la BMCE. Elle me dit: il faut voir ces gens-là.» (Entretien avec Fahd Yata, 2017)

Si ces expériences pionnières de la constitution d'entreprises de presse au Maroc montrent les conditions de possibilité de leur pérennisation, notamment à travers leurs relations avec les fractions dominantes du champ du pouvoir, elles pointent en filigrane un autre trait spécifique de l'univers journalistique marocain qui mériterait également d'être approfondi. En effet, cet univers professionnel était jusqu'au début des années 1990 dominé par le journalisme politique partisan et officiel, tout 
particulièrement ses éditorialistes, souvent chefs de parti et/ou porteparoles des autorités. Les journalistes entraient en effet via la presse partisane et étaient souvent issus, au moins pour les plus visibles, des filières traditionnelles du droit, de la science politique ou encore des langues et de la littérature.

Le journalisme et l'information économiques fournissent une autre voie d'entrée privilégiée, même si elle reste très probablement faible quantitativement. En effet, ceux-ci ont été non seulement I'un des principaux terrains de développement de la presse privée non partisane, mais aussi paradoxalement d'expérimentation du reportage politique et/ ou économique et/ou social. Au-delà de Nadia Salah et d'Afifa Dassouli, une série de figures importantes du journalisme au Maroc a débuté via la presse et/ou le journalisme économiques après avoir suivi des formations spécialisées, voire travaillé dans le domaine. C'est le cas de certains journalistes-entrepreneurs (nés à la fin des années 1960 ou au début des années 1970) des hebdomadaires généralistes «indépendants» - Le Journal (1997-2010) ou TelQuel: Ali Amar, I'actuel responsable du Desk, un site d'information ayant une dominante économique, son ancien associé au Journal Aboubakr Jamaï ou encore Ahmed Reda Benchemsi, qui sont tous les deux sortis de la profession et exercent en 2021 leur activité professionnelle à l'étranger. Dans la plus jeune génération, Aïcha Akalay, qui a dirigé TelQuel (2016-2019) et contribué au lancement de sa plateforme numérique, ou Omar Radi, qui a travaillé pour Le Desk et Médias 24 en se spécialisant dans les "enquêtes» et les "reportages», ont également suivi des filières économiques. D'autres ont fait carrière dans cette presse ou dans les rubriques ad hoc comme Naceureddine Elafrite, un patron de presse tunisien aujourd'hui à la tête de Médias24, un site d'information économique qu'il a lancé en 2013, après avoir occupé des responsabilités à L'Économiste à ses débuts, mais aussi à La Vie économique notamment. C'est également le cas de Mehdi Michbal (Finance News, Le Desk, Médias24, TelQuel, etc.) ou encore de Nabila Fathi (Le Journal, Challenges, Médias24, La Vie économique notamment), qui travaillent en 2021 depuis l'étranger.

Ce récit de deux expériences de développement d'entreprises de presse et de journalisme économique n'est cependant qu'un point d'entrée parmi d'autres d'une analyse de l'espace de la presse (papier et électronique) et de ses dirigeants qui reste à faire. En effet, l'étude des trajectoires des entreprises et de leurs principaux cadres dirigeants demeure pour l'instant un des points aveugles des enquêtes sur le sujet. 


\section{Bibliographie}

AIT-Mous Fadma, ZNAGUI Bachir, TRITKI Khalid, ABDounI Abdeljabbar, KSIKES Driss, Médias économiques et construction de la citoyenneté au Maroc: comportement des éditeurs et des journalistes, Rabat, CESEM, 2014.

BENABID Mohamed, Pratiques de consommation et processus de changement organisationnel: cas du marché de l'information en ligne, doctorat en cotutelle en sciences de l'information et de la communication, Université Paris 8, et en sciences de gestion, ISCAE, 2018. https://www.theses. fr/2018PA080044.pdf

BenChenNa Abdel, KsIKEs Driss, MARCHETTI Dominique, «La presse au Maroc: une économie très politique: le cas des supports papier et électronique depuis le début des années $90 »$, Questions de communication, n 32, 2017. DOI: https://doi.org/10.4000/questionsdecommunication.11527

BenCHENNA Abdel, MARChETTI Dominique, "Écrire entre les "lignes rouges": l'espace de la presse électronique au Maroc et ses enjeux», in Bruno Péquignot (dir.), Les Industries culturelles et créatives dans la mondialisation, Paris, L'Harmattan, 2019, coll. «Questions contemporaines», série "Les jeudis de L'Harmattan ». https://hal.archives-ouvertes.fr/hal-02109002v1

BENCHENNA Abdel, MARCHETTI Dominique, «Une offre sous conditions: les logiques contemporaines du champ du pouvoir marocain pour contrôler la presse nationale», in Yauheni Kryzhanouski, Dominique Marchetti et Bella Ostromooukhova (dir.), L'Invisibilisation de la censure: les nouveaux modes de contrôle des productions culturelles: Biélorussie, France, Maroc et Russie, Paris, Eur'Orbem éditions, Études et travaux, 2020. https://halshs. archives-ouvertes.fr/halshs-03096109v2

BENNANI-ChRAÏBI Mounia "Le Maroc à I'épr euve du temps mondial », dans Le Temps mondial, sous la dir. de Z. Laidi, Bruxelles, Complexe, 1997.

BENNANI-ChraïBI Mounia, "Hommes d'affaires" versus "profs de fac": la notabilisation parlementaire d'un parti de militants au Maroc», Revue internationale de politique comparée, 2008, vol. 15, n² 2. DOI: https://doi. org/10.3917/ripc.152.0205

BenSLIMANE Mehdi, 2015, Presse «indépendante» et pouvoir: le Journal (19972010) promoteur du trône au Maroc: une psycho-socio-anthropologie historique du journalisme politique, thèse en science politique, Université de Grenoble-Alpes. https://tel.archives-ouvertes.fr/tel-01459214/ document 
BENSMAIN Abdallah, 2015, Alors I'information? Les journalistes parlent du journalisme... et d'eux-mêmes, Casablanca, Afrique-Orient.

Bouderbala Négib, "La lutte contre la corruption: le cas du Maroc», Critique économique, 2001. https://revues.imist.ma/index.php/CE/article/ download/2650/1931

CATUSSE Myriam, «L'entrepreneur politique dans la réforme: discours et praxis de la libéralisation », Hespéris-Tamuda, n XXXIX, 2001.

CATUSSE Myriam, «A propos de «l'entrée en politique» des «entrepreneurs » marocains ", NAQD, $\mathrm{n}^{\text {os }} 19-20,2004$. DOI : https://doi.org/10.3917/ naqd.019.0127

CATUSSE Myriam, Le Temps des entrepreneurs? Politique et transformations du capitalisme au Maroc, Paris, Institut de recherche sur le Maghreb contemporain-Maisonneuve \& Larose, 2008 (éd. électronique 2014). DOI: https://doi.org/10.4000/books.irmc.525

Duval Julien, Critique de la raison journalistique: les transformations de la presse économique en France, Paris, Seuil, 2004.

EL MALKI Habib, «Crise économique et financière: austérité et FMI: le cas du Maroc ", Afrique et Développement, vol. 10, n ${ }^{\text {os }} 1-2,1985$. https://www. jstor.org/stable/24487207

HANNACHI Mariem, La Régénération stratégique de l'entreprise familiale : le cas des entreprises multigénérationnelles, thèse en sciences de gestion, Université d'Avignon, 2015.

HARI Tarik, PÉRALDI Michel, TOZY Mohamed, «Le patronat marocain: action collective, représentation de défense des intérêts", Revue Économie, Gestion et Société, n 16, 2018, https://revues.imist.ma/index.php/REGS/ article/view/12631/7048

HıBOU Béatrice, Tozy Mohamed, «De la friture sur la ligne des réformes: la libéralisation des télécommunications au Maroc », Critique internationale, n 14, 2002. DOI: https://doi.org/10.3917/crii.014.0091

HıBOU Béatrice, «Nous ne prendrons pas le maquis : entrepreneurs et politique en Tunisie», Politix, n 84, 2008. DOI: https://doi.org/10.3917/ pox.084.0115

HIBOU Béatrice, Tozy Mohamed, Tisser le temps politique au Maroc: imaginaire de l'État à l'âge néolibéral, Paris, Karthala, 2020.

IDDINS Annemarie, «Economic Life: global capital, financial journalism, and independent media ", Media, Culture \& Society, 2021. DOI : https://doi. org/10.1177\%2F0163443720986024 
KAIOUA Abdelkader, "Les grosses fortunes économiques et industrielles à Casablanca », Cahiers de la Méditerranée, nºs 46-47, 1993. DOI: https://doi. org/10.3406/camed.1993.1660

Kraemer Gilles, La Presse francophone en Méditerranée, Paris, Maisonneuve \& Larose, 2001.

KSIKES Driss, "Chronique des liens contrastés entre médias et pouvoirs au Maroc», Economia.ma, n²1, 2014. http://economia.ma/content/ chronique-de-liens-contrast\%C3\%A9s-entre-m\%C3\%A9dias-et-pouvoirsau-maroc

MARCHETTI Dominique, "Les nouveaux rapports de forces entre "grossistes" de l'information internationale: le cas révélateur de la production et de la circulation transnationale de l'information journalistique «marocaine», in Dominique Marchetti (dir. avec la collaboration de Julien Paris), La Circulation des productions culturelles: cinémas, informations et séries télévisées dans les mondes arabes et musulmans, Rabat-Istanbul, éditions électroniques du CJB et de I'IFEA, 2017. DOI: https://doi.org/10.4000/ books.cjb.1218

OUBENAL Mohamed, ZeROUAL Abdellatif, «Gouverner par la gouvernance: les nouvelles modalités du contrôle politique des élites économiques au Maroc », Critique internationale, n 74, 2017. DOI : https://doi.org/10.3917/ crii.074.0009

RousSILLON Alain, «La presse économique marocaine saisie par les marchés», Critique économique, $n^{\circ} 2$, 2001. https://revues.imist.ma/index.php/CE/ article/viewFile/2634/1915

\section{Sources}

AHOUGNON Servan, «De chef d'entreprise à chef du gouvernement, le parcours doré d'Aziz Akhannouch», Agence Ecofin, 17 septembre 2021. https:// www.agenceecofin.com/portrait/1709-91609-de-chef-d-entreprise-achef-du-gouvernement-le-parcours-dore-d-aziz-akhannouch

ASSARAF Robert, «La presse dans tous ses états», 21 novembre 2005. https:// www.jeuneafrique.com/84348/archives-thematique/la-presse-danstous-ses-tats/

BELYAZID Khalid, "Les 30 ans de L'Économiste: comment est née et s'est développée l'institution Éco-Médias», L'Économiste, 29 octobre 2021.

BERRAOUI Jamal, «Qui est le patron des patrons marocains?», Jeune Afrique, 12 juin 2006. 
Boum Aomar, PARK Thomas Kerlin, Historical dictionary of Morocco, 3e édition, Lanham, Maryland, Rowman \& Littlefield, 2016.

CRÉTOIS Jules, «Dix choses à savoir sur Driss Jettou, le président de la Cour des comptes du Maroc », Jeune Afrique, 12 septembre 2018. https://www. jeuneafrique.com/mag/626442/politique/dix-choses-a-savoir-sur-drissjettou-le-president-de-la-cour-des-comptes-du-maroc/

Douyeb Mohamed, "La saga des Kettani", La Gazette du Maroc, $1^{\text {er }}$ décembre 2003. https://www.maghress.com/fr/lagazette/3724

DUTEIL Mireille, "Moulay Hafid Elalamy, serial entrepreneur», Le Point, 19 octobre 2014.

HACHIM Mouna, Dictionnaire des noms de famille du Maroc: histoires et légendes, Casablanca, Auto-édition, 2006.

HOPQUIN Benoît, "Le résistant Charles Gonard est mort", Le Monde, 12 juin 2016. https://www.lemonde.fr/disparitions/article/2016/06/13/ le-resistant-charles-gonard-est-mort_4948993_3382.html

ISLAH Fadwa, "Hommage: Jean-Louis Servan-Schreiber, le Marocain», Jeune Afrique, $1^{\text {er }}$ décembre 2020.

L'ÉCONOMISTE, "Regard sur l'actualité », 14 novembre 1996. https://www. leconomiste.com/article/regard-sur-lactualite-247?destination=node/777898

L'ÉCONOMISTE, "L'ÉConomiste crée une direction du développement", 16 octobre 1997. https://www.leconomiste.com/article/leconomiste-creeune-direction-du-developpement

L'ÉCONOMISTE, «Nouveau conseil d'administration: un team d'enfer!», 5 novembre 1999. https://www.leconomiste.com/article/h2-nouveauconseil-dadministration-h2-centerun-team-denfercenter

L'ÉCONOMISTE, "Les 15 ans de La Nouvelle Tribune», 27 janvier 2011. https:// www.leconomiste.com/article/breves-1725

L'ÉCONOMISTE, «Khalid Belyazid: directeur général», 3 août 2016. https://www. leconomiste.com/membres-eco/khalid-belyazid-directeur-general

ÉCO-MÉDIAS, «Continuité», L'Économiste, 4 janvier 2021.

LE DESK, Reporters sans frontières (RSF), Media Ownership Maroc, 2021. https://maroc.mom-rsf.org/fr/proprietaires/proprietaires-individuels/ detail/owner/owner/show/afifa-dassouli/

LE DESK, "Décès de Jean-Louis Servan-Schreiber, ancien patron de La Vie Éco », 29 novembre 2020. https://ledesk.ma/encontinu/deces-de-jeanlouis-servan-schreiber-ancien-patron-de-la-vie-eco/ 
LES ÉCHOS, "L'ONA et Salomon Brothers lancent un fonds marocain », 7 avril 1994.

M.A.B., «Abdelaziz Alami : I'itinéraire d'un banquier poète», L'Économiste, 13 mai 2014. https://www.leconomiste.com/article/946741-abdelazizalami-I-itineraire-d-un-banquier-poete

MAGHREB CONFIDENTIEL, «Mohammed VI freine "Citizen Kane" Aziz Akhannouch», $\mathrm{n}^{\circ} 1336,18$ avril 2019.

MANYANI Hassan, "Abderrahmane Saaidi n'est plus!», Challenges.ma, 12 mai 2020. https://www.challenge.ma/abderrahmane-saaidi-nestplus-140142/

MAROC HEBDO, «Décès de Abderrahmane Saaidi, ancien ministre », 12 mai 2020. https://www.maroc-hebdo.press.ma/deces-abderrahmane-saaidi

OIKNINE Laurence, MRANI Omar, "Afifa Yata: Fahd aurait été un grand homme sans moi», VH Magazine, mars 2011. https://fr.calameo.com/ $\mathrm{read} / 005014854 \mathrm{~d} 5 \mathrm{fcdc} 3863 \mathrm{f0}$

SABIB Bouchra, «Najmat Bladi, vibrant hommage aux femmes», L'Économiste, 9 mars 2012. https://www.leconomiste.com/article/892143-najmat-bladivibrant-hommage-aux-femmes

SALAH Nadia, "Salomon après Soros, le coup double de l'ONA ", L'Économiste, 7 avril 1994. https://www.leconomiste.com/article/salomon-apres-sorosle-coup-double-de-lona

TovI Laurence, "Un patron des patrons qui n'a pas froid aux yeux», Les Échos, 7 septembre 2007. 
Partie III

Les programmes français d'«aide aux médias » et leurs appropriations: le cas de la Tunisie 



\title{
Chapitre 7 \\ La télévision étatique tunisienne, un espace d'expérimentation de la «transition médiatique » au lendemain de 2011 \\ Le rôle-clé des acteurs français de «l'aide aux médias »
}

\author{
Chirine Ben Abdallah \\ "Connaître le passé est une manière de s'en libérer.» \\ Raymond Aron, Dimensions de la conscience historique, \\ Paris, Les Belles Lettres, 2017
}

Les transformations politiques survenues en Tunisie à la suite du renversement du régime Ben Ali en janvier 2011 marquent théoriquement la fin du contrôle de l'information par l'État ${ }^{1}$. Les journalistes de la télévision publique se sont cependant retrouvés désarmés face à ce chamboulement, ayant longtemps vécu et travaillé sous le joug de la censure. Si celle-ci a été levée au sein de la télévision nationale, la liberté d'expression acquise pendant la «Révolution de 2011 » s'avère être une arme à double tranchant: d'une part, elle reste fragile, comme en témoignent les pressions et les intimidations subies par certains journalistes et rédacteurs en chef $^{2}$ des journaux télévisés

\footnotetext{
${ }^{1}$ Le présent chapitre est extrait d'une thèse de doctorat réalisée entre 2011 et 2015 et intitulée "Les nouveaux enjeux de l'information à la télévision tunisienne en situation de transition sociopolitique : la complexité des partenariats France-Tunisie au lendemain de janvier 2011 ». ${ }^{2}$ Certains journalistes ont été victimes d'intimidations, comme ce fut le cas de la journaliste Naima Jouini, la présentatrice-phare du 20 heures d'avant janvier 2010, ayant reçu une distinction sous Ben Ali. En juin 2018, elle a été sermonnée publiquement par Béji Caid Essebsi, alors Premier ministre par intérim, qui a refusé de répondre à sa question sur les mouvements de grève dans le pays. La journaliste a même eu droit à une contre-question de la part d'Essebsi, qui lui a demandé son âge et lui a rétorqué: « Vous ne faites pas de l'information, je sais quelles sont vos arrière-pensées et connais très bien votre clan. » L'agression été fortement relayée sur
} 
sous les différents gouvernements dits "de transition »; d'autre part, c'est une source de conflits en raison des dénonciations récurrentes, du «manque d'impartialité » et des «dérapages» de certains professionnels ${ }^{3}$.

Dans ce contexte, plusieurs acteurs internationaux (ONG, groupements de bailleurs, diplomates) ont proposé leur appui aux autorités tunisiennes pour accompagner les chaînes publiques dans leur processus de passage de médias «au service du régime» vers des médias "de service public ${ }^{4}$ ». Considérant l'indépendance et la professionnalisation des organes de presse comme un prérequis pour la réussite de la «transition» et la "consolidation de la démocratie», la France, partenaire historique, a proposé diverses formules pour contribuer à la professionnalisation du secteur journalistique tunisien. D'après nos interviewés, responsables de la coopération française en Tunisie post-14 janvier 2011, celle-ci ne viserait plus à imposer ses stratégies, mais plutôt à assurer un «accompagnement». Sur le terrain, les notions de «réciprocité » et d'«échange d'égal à égal » sont les maîtres-mots de la diplomatie culturelle, comme le montrent par exemple certaines collaborations de journalistes tunisiens à des programmes français s'intéressant à la Tunisie ${ }^{5}$. Ce chapitre se propose de restituer les relations de coopération au cœur du processus du changement politique en Tunisie, et plus particulièrement les partenariats franco-tunisiens, en analysant le rôle et la stratégie des acteurs de l'audiovisuel, c'est-à-dire les chaînes

les réseaux sociaux et a tourné en boucle à la télévision nationale, qui a déploré l'intervention du Premier ministre et a rappelé son attachement aux règles déontologiques et à l'impartialité face aux tentatives d'instrumentalisation.

${ }^{3}$ Mokhtar Rassâa, directeur général par intérim de la Télévision tunisienne, témoigne dans un entretien qu'il nous accordé des dérapages et du manque de rigueur de certains journalistes du JT dans la préparation de certains sujets. Ainsi, dans un reportage diffusé au lendemain de l'annonce des résultats des élections à l'Assemblée nationale constituante (ANC), la voix off de H. Ghidaoui précisait : «En choisissant d'élire Ennahdha, le peuple a choisi la voie de I'islam. » De même, la journaliste Fatin Hafsia a accusé sans fondements dans un reportage un ministre du gouvernement d'Ennahdha d'être impliqué avec l'ancien régime. Mokhtar Rassâa a choisi de ne pas sanctionner sévèrement les manquements aux règles de la déontologie journalistique, compte tenu du contexte et du fait que ces journalistes avaient besoin de temps pour transformer leurs pratiques.

${ }^{4}$ Nous avons entamé notre travail de terrain très tôt, plus précisément dès 2011. Quelques chercheurs se sont intéressés par la suite à la question, entre autres Mohamed Ali Elhaou et Aïda Fitouri (2015), Olivier Koch (2017) ou encore Zeineb Touati (2017).

${ }^{5}$ L'expérience nouvelle de faire participer des journalistes des pays de la réception, à savoir faire appel à des journalistes tunisiennes pour préparer des reportages de numéros spéciaux sur la Tunisie de l'émission «Maghreb-Orient Express» sur TV5Monde, pourrait être classée parmi les actions de réciprocité plus que de coopération. 
françaises et tunisiennes, ainsi que les actions d'accompagnement menées par les différents partenaires français.

La présente recherche, qui se situe au carrefour de la sociologie de la communication et de la sociologie politique, privilégie une approche compréhensive, permettant de saisir les logiques sous-jacentes aux pratiques des acteurs de l'audiovisuel. La phase de recueil des entretiens n'a pas toujours été facile en raison du contexte politique et médiatique instable. Le choix d'interroger des journalistes et des acteurs médiatiques de divers horizons est délibéré (42 interviewés), afin de recueillir des témoignages sur le style de management des directeurs, le fonctionnement du service d'information à la télévision publique tunisienne ou encore sur les modalités de l'intervention française au lendemain de la «révolution».

Cette enquête réalisée dans le cadre d'une thèse s'appuie essentiellement sur des entretiens en Tunisie $(n=24)$ et en France $(n=18)$, qui ont été réalisés entre 2011 et 2013. Dans un premier temps, les interviews ont été menées en France auprès de plusieurs acteurs, dont des formateurs ayant participé aux programmes en Tunisie, des cadres de Canal France International, de l'Académie France 24, des responsables de la coopération de France Télévisions, TV5Monde, France 24, des diplomates en charge de l'audiovisuel français, deux responsables de la Direction générale des médias et des industries culturelles (DGMIC), le directeur du Centre national de la cinématographie (CNC) et des consultants. Dans un second temps, les entretiens ont été réalisés avec des professionnels et des dirigeants de différentes structures de l'audiovisuel tels que des membres d'institutions de formation (Centre africain de perfectionnement des journalistes et communicateurs, CAPJC), de régulation (Instance indépendante chargée de réformer l'information et la communication, INRIC), des journalistes de télévision (cadres dirigeants, journalistes, présentateurs, chargés des échanges audiovisuels) et des cyber-activistes.

\section{La télévision au cœur des transformations sociopolitiques de la Tunisie}

\section{La télévision tunisienne d'avant le 14 janvier 2011 et ses modes de contrôle: de Bourguiba à Ben Ali}

Depuis l'Indépendance, les médias ont toujours constitué une préoccupation politique essentielle des dirigeants politiques tunisiens. Instrument de pouvoir, l'information était également présentée comme un moyen, un véhicule du conditionnement socio-politique, ou encore comme 
une «école parallèle» (Lerner, 1958, p. 28) pour le développement, l'unité et la souveraineté nationales, tout du moins pour Habib Bourguiba. Sous Zine el-Abidine Ben Ali, l'information a été investie essentiellement de la mission de porte-parole de l'appareil propagandiste.

Très tôt, ce dernier a en effet fait de la maîtrise des médias son cheval de bataille. Pour asseoir son pouvoir, il a non seulement veillé au développement de la radio, comme l'avait fait son prédécesseur, mais c'est aussi et surtout la télévision qui a occupé une place de choix dans ses stratégies de communication. L'ouverture au secteur privé a touché essentiellement le paysage radiophonique, mais elle n'a été que factice. Sous Bourguiba, l'espace médiatique était en quelque sorte plus uniforme et moins ambigu. Le champ d'intervention présidentiel était limité aux médias d'État, qui étaient investis pour servir le régime en place. Néanmoins, des périodes d'ouverture politique relative étaient envisageables ${ }^{6}$. En revanche, Ben Ali a opéré un contrôle médiatique total et a tout mis en œuvre pour qu'il soit impossible à transgresser. L'ouverture médiatique affichée avec les autorisations de diffusion données à des chaînes de télévisions privées, telles que Hannibal TV et Nessma ${ }^{7}$, ou à des radios privées ${ }^{8}$ a créé une dynamique dans le secteur de l'audiovisuel. En effet, de nouvelles productions télévisuelles sont apparues, le plurilinguisme (Achour Kallel, 2011) s'est développé avec Mosaïque FM, et de nouveaux genres ont émergé

\footnotetext{
${ }^{6}$ Les plus progressistes parmi les proches de Bourguiba tentaient sans cesse de couper avec le monolithisme qui ternissait l'image du parti. C'est dans cette perspective qu'Omar S'Habou, rédacteur en chef d'une publication du Parti socialiste destourien (PSD), et Mohamed Sayeh, haut cadre dirigeant du PSD, ont pris l'initiative de lancer Dialogue, un journal d'opinion, ce projet suscitant une polémique au sein du parti au pouvoir, alors même que Bourguiba le soutenait. Après deux années de "perles révolutionnaires qui ont mis le parti sens dessusdessous » comme le déclare Omar S'habou, la tension est devenue telle entre les membres de Dialogue et le pouvoir bureaucratique, les plus conservateurs du parti destourien, que l'équipe du journal a présenté sa démission collective.

${ }^{7}$ Le choix de Larbi Nasra, proche de la famille de la Première dame, Leila Trabelsi, comme promoteur de la première télévision privée, Hannibal TV, ainsi que l'octroi de l'autorisation de diffusion ont été décidés par le président Ben Ali en personne. Une deuxième autorisation a été accordée plus tard à l'agence de communication Karoui \& Karoui, très présente au Maghreb central depuis plusieurs années, pour lancer le 15 mars 2007 Nessma TV, une télévision satellitaire baptisée La chaîne du Maghreb.

${ }^{8}$ Dans un discours prononcé le 7 novembre 2003, Ben Ali annonce aux radios et télévisions du secteur privé la possibilité d'émettre ainsi que «l'attribution d'une fréquence » et le démarrage de «la première station radio privée » à transmission indépendante en FM, sur le Grand Tunis: Radio Mosaïque. Une deuxième station privée, régionale, Radio Jawhara FM (la perle du Sahel), est autorisée à émettre à partir de juillet 2005, d'autres radios ont vu le jour par la suite: Shems FM, Express FM et Zitouna FM.
} 
sous l'impulsion des radios thématiques comme Zitouna FM (religieuse) et Express FM (économique). Pourtant, en aucun cas ces nouveaux supports n'ont été indépendants ou libres dans leur offre éditoriale ou même leur gestion. La libéralisation du secteur audiovisuel, amorcée par Ben Ali, s'est trouvée ainsi «fondue dans le moule étatique»(Chouikha, 2012, p. 2).

L'analyse des productions éditoriales de la chaîne nationale, et plus précisément de son journal télévisé (JT), résume à grands traits cette période de forte dépendance. Le JT a en effet été instrumentalisé, comme en témoigne Nejah Missaoui ${ }^{9}$, journaliste et chef d'édition du journal, pour servir le régime en place et redorer son image.

«II n'y avait vraiment pas de ligne éditoriale dans le JT, tout l'intérêt était porté sur le président et sur son gouvernement, le reste on s'en moque... ॥ y a même certains sportifs qui sont censurés; on ne doit pas les voir à l'écran ou en parler à cause de problèmes qu'ils peuvent avoir avec le régime en place. Gare au journaliste qui ose transgresser la règle, II faut dire qu'avec tous ces obstacles, l'autocensure a gagné les journalistes, ils ne font même plus d'effort pour pousser les marges de la liberté, au point qu'elle est devenue innée chez eux. » (Entretien, février 2011, Tunis)

Selon le rapport de l'Institut Panos réalisé en 2012 (Institut Panos Paris et Observatoire méditerranéen de la communication, 2012, p. 123), les images du président occupaient $22,03 \%$ du temps d'image. Le régime autoritaire de Ben Ali a été marqué par ce que Georges Balandier (1980, p. 151) appelle un «déploiement cérémoniel», une "théâtralisation» des grandes manifestations collectives, c'est-à-dire une politique de l'image qui emprunte nécessairement à l'art du spectacle ${ }^{10}$.

Le processus de transnationalisation télévisuel avec le développement de la diffusion des chaînes étrangères via les satellites dans les années 90 a été un premier pas vers la démonopolisation de la programmation

\footnotetext{
${ }^{9}$ Nejah Missaoui a été rédacteur en chef et producteur-présentateur des débats politiques dans les dernières années de Ben Ali. Il est reconnu pour être proche du régime sans pour autant occuper de poste politique au sein du RCD.

${ }^{10}$ Les fêtes du 7 novembre sont au cœur de la politique de l'image et du spectacle du régime de Ben Ali. Elle a été mise en place notamment par l'homme fort du régime, Abdelawahab Abdallah, qui a débuté à partir de 1990 comme conseiller politique et de l'information du président de la République après avoir occupé différents postes-clés dans les ministères de la Culture, de I'Information et des Affaires étrangères, aussi bien sous Bourguiba que sous Ben Ali. Signe de tout régime totalitaire, l'information fut dans ce système toujours officielle et contrôlée par les autorités politiques. Les sujets tabous n'ont cessé de s'élargir, préparant le terrain à la mise en place d'un système de contrôle tentaculaire sur fond de flou juridique et de répression en matière d'information et de presse.
} 
télévisuelle et a constitué un élément déstabilisateur pour le régime. C'est ce qui explique sa politique de limitation de l'accès à ces chaînes. Pendant toute la période de gestation allant du 17 décembre 2010 (début des manifestations) au 13 janvier 2011 (la veille de la fuite du président déchu Ben Ali), les médias tunisiens ont totalement ignoré les contestations ou les ont considérées comme des faits isolés initiés par une minorité d'«agitateurs». Par exemple, des documentaires animaliers étaient programmés au moment des émeutes.

Le régime de Ben Ali ayant interdit les journaux télévisés sur les chaînes privées pour garantir un meilleur contrôle de l'information, la chaîne nationale TV7 proposait le seul rendez-vous télévisé des Tunisiens avec l'information, officiellement crédité d'une audience de $18 \%$. D'après le sociologue Riadh Ferjani, des sources officieuses parlaient plutôt de 1 à $2 \%$, tant ce JT était discrédité auprès de la population, et il ajoutait: «On le regardait plus pour se tenir au courant de la comédie du pouvoir, la météo même était sujette à caution!» (Ferjani, 2002, p. 158) C'est ainsi que le JT de TV7 a attendu une semaine pour rompre le silence: le 24 décembre, les événements de Sidi Bouzid sont évoqués. Cependant, les journalistes en charge du journal ne se sont attardés ni sur l'évolution des événements ni sur le nombre de morts, mais ils ont uniquement diffusé des images de banques saccagées et de véhicules de police vandalisés. Aucune image de la répression policière n'a été proposée à l'antenne, la parole étant donnée à des gens qui déplorent les actes de violence en réduisant le mouvement social et politique à de simples actes de vandalisme. Une attention particulière a par contre été accordée au discours du président et à sa visite à Mohamed Bouazizi, un marchand ambulant qui s'était immolé par le feu en signe de protestation suite à une altercation avec un agent municipal.

Contrairement aux crises qui ont précédé ${ }^{11}$, et qui ont toujours été étouffées par le système de censure sous Ben Ali, les événements de décembre 2010 ont échappé au contrôle des médias et aux moyens de communication classiques. Leur diffusion trouvant refuge sur internet, les manifestants ont contourné les mécanismes de verrouillage mis en place par l'Agence tunisienne d'internet (ATI). Dans un pays où les «caractéristiques de l'organisation technique et administrative d'internet permettaient à l'État

\footnotetext{
${ }^{11}$ La censure était de mise à la télévision tunisienne dès qu'il s'agissait d'un sujet sensible, tels les procès politiques, la fusillade de Slimane en 2006 ayant opposé les forces de l'ordre à un groupe armé djihadiste ou encore le soulèvement des ouvriers du bassin minier de Gafsa en 2008.
} 
d'exercer un contrôle rigoureux sur les usages de la toile» (Bras, 2000, p. 36), les mouvements de protestation ont trouvé dans les réseaux sociaux une réelle caisse de résonnance pour diffuser leurs messages de détresse, qui ont été relayés par la suite par les médias étrangers, marquant ainsi la fin de ce que Larbi Chouikha appelle "l'étatisation de l'information» (Chouikha, 1992, p. 72).

La prise en charge croissante de la diffusion médiatique par l'ensemble des acteurs sociaux en Tunisie a fait d'internet et de quelques réseaux sociaux des espaces privilégiés de participation citoyenne pour les Tunisiens, qui a fait notamment émerger des "auto-médias», selon l'expression de Pierre Lévy (Lévy, 2002, p. 43). En effet, les médias ne s'adressent plus seulement à des individus, mais aussi à des médias à part entière. La mobilisation des cyberactivistes a été déterminante pour réussir l'imbrication entre le virtuel et le terrain dans la révolution tunisienne. Comme l'explique l'un d'entre eux, Haythem Mekki ${ }^{12}$, les pages et profils de dissidents sont désormais des médias, des récepteurs, mais ils produisent et recommandent aussi des contenus (Lecomte, 2009).

\section{Les chaînes transnationales, une caisse de résonnance pour les Tunisiens indignés}

En plus du rôle joué par internet et les réseaux sociaux, les télévisions transnationales ont porté la voix des Tunisiens indignés et relayé à l'étranger les mouvements de contestation de décembre 2010. Deux chaînes transnationales ont retenu notre attention par leur couverture des révoltes sociales en Tunisie: d'un côté, Al Jazeera, une des chaînes satellitaires en langue arabe les plus regardées lors de la "révolution tunisienne»; de l'autre, France 24, une chaîne francophone qui nous éclaire sur les relations franco-tunisiennes et, pour reprendre l'expression officielle, sur la «vision française de l'information internationale ».

À l'image des chaînes anglophones telle que $B B C$ World, la France veut faire porter sa voix par une chaîne d'information internationale, France 24, dont les versions francophone et anglophone ont été créée le 6 décembre 2006. Cette nécessité apparaît d'autant plus forte pour les autorités politiques françaises dès les débuts de la guerre d'Irak, qui commence en 2003 et donne à voir la prédominance très forte dans la

\footnotetext{
12 Entretien avec Haythem El Mekki, bloggeur connu sous le pseudo Bylasko, converti en journaliste au lendemain de la Révolution de 2011, Tunis, le 14 février 2012.
} 
production de l'information internationale de CNN International et BBC World en langue anglaise, et d'Al Jazeera en langue arabe. France 24 lance donc à partir du 2 avril 2007 des programmes dans cette langue de 16 heures à 20 heures avant de devenir une chaine en continu, qui s'adresse notamment aux publics de la zone Maghreb, du Proche et du Moyen-Orient. Selon Philippe Di Nacera, directeur de France 24, la chaîne francophone, «la voix de la France ne veut pas dire celle du gouvernement français, mais l'idée est de montrer la façon française de regarder le monde, on fait notre travail de journaliste, mais dans la feuille de route de départ, ce qui nous est demandé c'est de regarder le monde d'où nous sommes, de France, d'Europe» (Entretien, mai 2011, Paris).

\section{La forte implication de France $\mathbf{2 4}$ dans la couverture de la «révolution tunisienne»}

La spécificité de la ligne éditoriale de France 24 est de parler « de la France au monde» et de parler "du monde à la France», comme le suggèrent ses responsables. Il s'agit d'apporter une dimension internationale à l'information «à la française». En quelques années, France 24 est devenue l'un des plus importants médias d'information dans le monde. En décembre $2013^{13}$, la chaîne rassemblait chaque semaine près de 42 millions de téléspectateurs et totalisait plus de 14 millions de visites sur son site internet. Les débuts ont été difficiles, l'audience de la chaîne ne décollant pas en raison de différents problèmes: «inadaptation marquée aux préoccupations et centres d'intérêt des publics arabes, incertitudes sur la stratégie internationale de l'AEF sur son positionnement par rapport à TV5Monde et Radio France Internationale (RFI), soutien institutionnel public insuffisant alors que la chaîne est appelée à être la voix de la France à l'étranger.» (Guaaybess, 2012, p. 96) Les événements au Proche et au Moyen-Orient ont été un facteur déclencheur pour le lancement et le développement ${ }^{14}$ de France 24 en langue arabe, ce qui lui a permis de se déployer partout dans le monde. La chaîne a été propulsée par les événements survenus en Tunisie en 20102011 et a obtenu des taux d'audience considérables dans le pays. Selon Jean-Christophe Fleury, le directeur de la sous-direction de l'audiovisuel

\footnotetext{
${ }^{13} \mathrm{Cf}$. données citées dans cet article sur France 24: http://fr.wikipedia.org/wiki/France_24

${ }^{14}$ Deux ans après le lancement de la chaîne arabophone, celle-ci a vu son temps d'antenne quotidien augmenter significativement, passant de 4 heures de diffusion à 10 heures, diffusant de 14 heures à minuit. Le 12 octobre 2010 à midi heure française, le canal arabe de France 24 a commencé sa diffusion 24 heures sur 24.
} 
extérieur au sein du ministère des Affaires étrangères, «la consultation du site internet y a augmenté de près de $1000 \%$ », et la chaîne francophone occupait la deuxième place à cette époque-là après $A$ l Jazeera et bien devant CNN International et BBC World.

France 24 a commencé très tôt la couverture des événements en Tunisie, c'est-à-dire dès que le vendeur ambulant Mohamed Bouazizi s'est immolé par le feu au mois de décembre 2010. Contrairement aux chaînes nationales françaises, France 24 a accordé une forte importance aux événements depuis la première dépêche reçue à ce sujet. Sarra Grira, journaliste-rédactrice internationale au desk internet de la chaîne, qui faisait alors partie de l'équipe des observateurs, témoigne:

"Le 17 décembre une dépêche est sortie un vendredi après-midi pour dire qu'il y a eu un jeune qui s'est immolé par le feu, et qu'il y a eu des manifestations devant le bureau du gouvernorat de Sidi Bouzid. Le rédacteur en chef m'a chargée de vérifier l'information à travers nos observateurs, comme le champ médiatique est très verrouillé en Tunisie. Il se trouve que, quelques mois auparavant, il y a eu une affaire de terres confisquées par la banque agricole en Tunisie dans la région de Regueb, et j'avais fait un papier sur la question avec l'aide d'un observateur de la région. J'ai directement pensé à lui, d'autant plus qu'il est syndicaliste, il doit être informé. J'ai réussi à le joindre, il était sur place et comptait nous contacter. Il a proposé de nous filmer des séquences de ce qui se passait, qu'il a envoyées le soir même.» (Entretien, mai 2011, Paris)

Ces images ont été diffusées dans le JT du jour-même, et le lundi l'équipe des observateurs a publié un billet à propos de l'affaire sur le site de la chaîne. France 24 s'est intéressée dès ses débuts à des sujets plus sociaux et souvent éludés par les médias tunisiens, comme cette manifestation d'agriculteurs en août 2010 à Regueb, relayée grâce au syndicaliste Slimane Rouissi. Comme le montrent les audiences, elle a bénéficié d'un bon crédit chez les Tunisiens, au même titre que la chaîne Al Jazeera lorsque commence la couverture de la révolution fin 2010.

Durant la période du mois de décembre 2010 où les correspondants étrangers étaient interdits en Tunisie, France 24 a beaucoup compté sur l'apport de son programme "Les Observateurs». L'idée est de susciter auprès des téléspectateurs-observateurs intéressés qui regardent la chaîne des envois de témoignages sous forme de vidéos, de photos ou de textes. Au fil du temps, la chaîne a constitué un réseau de plusieurs milliers d'observateurs dans le monde. Une cellule de journalistes spécialisés dédiée à la vérification de ces informations a été mise en place. 


\section{Illustration 1}

\section{Une image de la couverture de France 24 des événements à Thala en décembre 2010 en Tunisie}

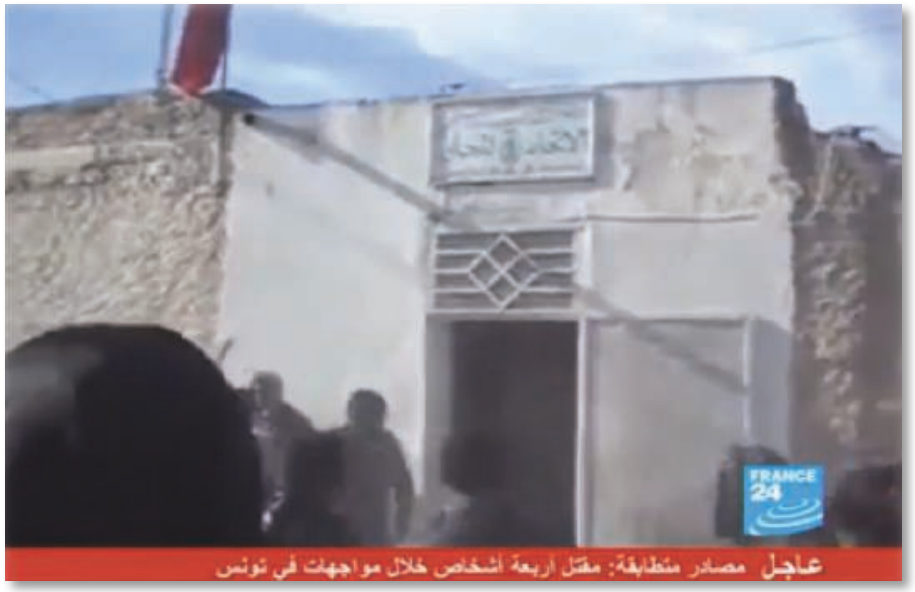

Source: capture d'écran de l'auteure.

Facebook a contribué au programme par la suite grâce aux publications des activistes et des amis des journalistes, surtout des Tunisiens, dont fait partie Sarra Grira, journaliste-rédactrice internationale, qui a exploité son carnet d'adresses pour le suivi de l'actualité de la répression en Tunisie.

«J'ai des amis bloggeurs opposants en Tunisie tels que Sofiène Chourabi ${ }^{15}$, Lina Ben Mhenni, Nejib Ayadi qui m'ont aidée et m'ont fourni des vidéos en exclusivité. Surtout Sofiène, qui a accepté, puisque c'est un ami, de nous donner ses vidéos en avant-première avant de les poster sur son compte Facebook. Les autres chaînes comme France 2 et TF1 ont relayé par la suite ces mêmes vidéos.» (Entretien, mai 2011, Paris)

Appuyée par les réseaux sociaux, France 24 a joué un rôle de caisse de résonnance extra-nationale, de contre-pouvoir, participant à la constitution d'un "espace de négociation du sens" » et de prise en charge des "préoccupations des groupes sociaux exclus», comme l'explique Jesús Martín-Barbero (cité par Cabedoche, 2007, p. 348).

\footnotetext{
${ }^{15}$ Au moment de la rédaction de notre thèse en août 2015 et jusqu'à ce jour, Sofiene Chourabi est toujours porté disparu en Libye avec son collègue et caméraman Nadhir Guetari suite à un déplacement pour une émission d'investigation sur les groupes terroristes dans la région du Maghreb.
} 
Des Tunisiens partagent alors des vidéos et des photos sur les réseaux sociaux avec cette conscience que leurs images pourraient être relayées pour renforcer l'impact des manifestations. "Petit à petit les gens ont commencé à nous connaître et à nous contacter, ils sont contents de voir que leurs vidéos sont relayées », explique encore Sarra Grira. Leur objectif est de faire entendre leur voix, et ceci passe par la diffusion de leur contenu: plus il est partagé, plus leur «cause » sera connue par un large public.

En effet, depuis les événements du 17 décembre 2010, la portée d'internet, en général, et des réseaux sociaux, en particulier, n'a cessé de se développer en Tunisie, et l'utilisation essentiellement ludique qui en était faite s'est accompagnée d'usages plus politiques. Les réseaux sociaux issus du web 2.0 sont devenus « des vecteurs d'un élargissement des solidarités en contrepoint des communautés plus fermées de l'époque précédente» (Leteinturier, 2013, p. 113). C'est d'autant plus le cas en Tunisie où ils contribuent à la formation d'un nouvel espace d'expérimentation politique. Toujours selon la journaliste de France 24, Sarra Grira, les images «amateures » jouissent d'une valeur ajoutée dans le contexte du verrouillage médiatique et des difficultés d'accès au terrain qui a caractérisé la Tunisie sous la dictature. Dans un autre cas de figure, où il existerait par exemple des images d'agence, le rôle d'un "journaliste citoyen » consisterait plus à vérifier l'information que produire l'image. Comme le soutient Bertrand Cabedoche, «certaines parties du monde n'accèdent à une couverture médiatique transnationale qu'à partir d'événements rares» (Cabedoche, 2007, p. 353). France 24 a orienté son attention vers l'Afrique, qui est souvent délaissée par les grands médias généralistes nationaux et transnationaux, et le monde arabe. Moins d'un an après le lancement de son module arabophone 24 heures sur 24 et 7 jours sur 7, la chaîne française s'était déjà imposée comme une chaîne de référence dans les pays du Maghreb, alors même qu'elle s'inscrit dans un environnement très concurrentiel et dominé par les deux principales chaînes arabophones transnationales, Al Jazeera et Al Arabiya. En effet, selon une étude TNS Sofres réalisée en 2011, «78\% des Tunisiens déclarent avoir regardé France 24 pour s'informer sur les événements politiques. Pour $29 \%$ d'entre eux, c'est même la chaîne qu'ils regardent le plus souvent. De plus, parmi ceux qui ont choisi de se tourner vers de nouvelles chaînes d'information à l'occasion de la crise tunisienne, ils sont $47 \%$ à avoir choisi France 24 alors qu'ils ne la regardaient pas avant » (Gonzales, 2011). La même étude concluait aussi en faveur du site internet de la chaîne d'information qui a généré $77 \%$ de visites d'internautes ayant consulté des sites d'information. Enfin, selon l'institut tunisien Sigma, entre janvier et février 2011, «France 24 a vu son audience quotidienne 
progresser - tout comme celle d'Al Arabiya - de 3,4\% à 4,7\%» (Gonzales, 2011), tandis qu'Al Jazeera aurait perdu près de trois points d'audience. Dans les pays du Maghreb, et notamment en Tunisie et en Algérie, France 24 apparaîtrait selon les experts de la région arabe «comme une alternative à $A /$ Jazeera dont on ne comprend pas toujours le jeu médiatique» (Gonzales, 2011).

Tableau 1

Audience des chaînes de télévision en Tunisie en 2011 (en \%)

\begin{tabular}{|r|l|r|}
\hline 1 & France 24 & 46,9 \\
\hline 2 & Al Jazeera & 39,4 \\
\hline 3 & Al Arabiya & 34,7 \\
\hline 4 & Wataniya & 19,8 \\
\hline 5 & Nessma TV & 9,6 \\
\hline 6 & Al Hurra & 9,3 \\
\hline 7 & Al Mustakillah TV & 7,3 \\
\hline 8 & BBC Arabic & 6,4 \\
\hline 9 & Hannibal & 5,2 \\
\hline 10 & Al Hiwar & 5,0 \\
\hline 11 & BBC World News & 2,6 \\
\hline 12 & CNN International & 2,0 \\
\hline 13 & Al Manar TV & 1,7 \\
\hline 14 & Euronews & 1,2 \\
\hline 15 & Al Alam TV & 1,2 \\
\hline 16 & Al Mehwar & 0,9 \\
\hline 17 & Al Masriya & 0,9 \\
\hline 18 & Nile TV & 0,9 \\
\hline
\end{tabular}

Source: TNS/Sofres, mars 2011, cité par Gonzales, 2011.

\section{Le journal télévisé de la chaîne nationale Watanya 1, l'espace d'expérimentation par excellence de la "transition médiatique »}

De la présidence de la chaîne nationale Wataniya 1 jusqu'aux simples journalistes, le journal télévisé a été un espace d'expérimentation et d'apprentissage des règles d'une information plus libre, à partir de la fuite de Ben Ali au soir du 14 janvier 2011. Entre un passé peu glorieux et un présent révolutionnaire, les journalistes se sont trouvés désemparés face à la vitesse à laquelle les événements se sont succédé. Par son importance et son audience, le JT de la première chaîne est de ce fait un observatoire des pratiques professionnelles dans ce nouveau contexte. 


\section{En immersion au sein du JT de la première chaîne nationale}

Afin de voir de près et de comprendre son fonctionnement, nous avons réalisé une observation d'une journée au cœur de la Newsroom et des entretiens avec les journalistes et les responsables de la chaîne. Avant le 14 janvier 2011, les journalistes attendaient les directives du ministère de I'Intérieur ou les instructions d'Abdelwahab Abdallahet à ses hommes pour connaître le contenu du JT. Depuis, les choses ont changé. Lors de sa première réunion avec les journalistes, Mokhtar Rassâa, alors directeur de la télévision nationale, expose sa vision aux journalistes: "La mission que je me suis fixée consiste à redresser l'institution de la télévision et non pas à m'occuper du JT, vous êtes les seuls responsables et garants de la qualité du JT.» (Entretien, mai 2011, Tunis)

Ainsi les journalistes ont-ils constitué un comité de rédaction, Moufida Hachani, la tête de liste, étant choisie comme rédactrice en chef. Depuis lors, l'élaboration du journal de 20 heures commence avec une conférence de rédaction vers 15 heures, lors de laquelle se décide entre les journalistes et le rédacteur en chef la hiérarchie des éléments et des reportages. C'est Said Khezami qui nous a reçu ce jour-là au sein de sa rédaction, dont il a pris les commandes en mars 2011 à la suite d'un appel à candidatures.

Force est de constater que, à partir des éditions ${ }^{16} \mathrm{du}$ JT que nous avons analysées, la hiérarchie des informations post-14 janvier est dictée par l'importance des nouvelles et non plus imposée verticalement par les dirigeants de la chaîne. En effet, les activités présidentielles ne sont plus privilégiées, et la nature même des sujets traités a changé, ceux-ci étant désormais basés davantage sur le critère de proximité et marqués par une distance affichée à l'égard des personnalités officielles et de leurs activités. Le journal télévisé de la chaîne nationale Watanya 1 accorde désormais une importance primordiale à la «parole citoyenne» par la présence des micros-trottoirs dans ses reportages. Les citoyens interrogés s'expriment plus librement sur les politiques publiques, déplorent l'inflation et critiquent les services publics, notamment en matière de transport. Ce vent de liberté ayant soufflé sur la rédaction marque un regain ${ }^{17}$ d'autonomie des journalistes, qui est perceptible à la fois sur la forme et sur le fond du JT.

\footnotetext{
${ }^{16}$ Une analyse de contenu de 5 éditions du journal télévisé représentatives des périodes des trois chefs de gouvernements de transition qui se sont succédé entre 2011 et 2013.

${ }^{17}$ Ce travail selon les normes journalistiques, détaché du joug de l'État et respectant la déontologie du métier, a permis fin février 2012 au JT d'être en tête des audiences en prime time avec un public de 1 à 2 million(s) de téléspectateurs.
} 
Le journal relate désormais les activités de la «société civile» au sens large (avocats, associations, ONG internationales). De même, les experts peuvent s'exprimer librement, occupant une place de choix dans les éditions du 20 heures. Enfin, les intervenants en plateau sont invités à présenter leur lecture en toute liberté, voire même à proposer des analyses critiques de la politique du gouvernement ainsi que de l'action des partis de l'opposition, ces derniers ayant également leur place à travers les reportages et la couverture de leurs événements. Cette liberté de ton a fait gagner aux médias un nombre considérable de téléspectateurs, comme en témoigne le graphique ci-dessous.

\section{Graphique 1}

\section{Enquête auprès d'un échantillon de téléspectateurs de la télévision en Tunisie}

Vous intéressiez-vous aux informations pendant l'époque du régime déchu?

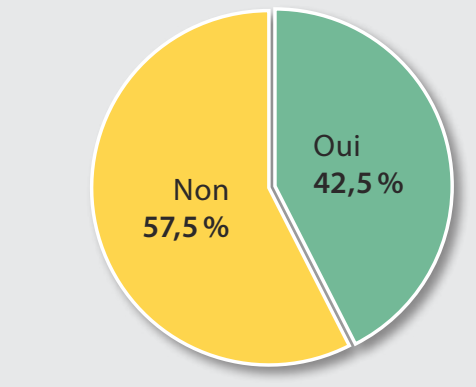

Vous intéressez-vous aux informations actuellement?

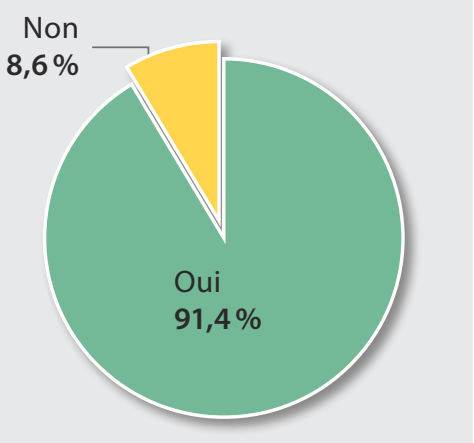

Source: ISTIS, 2011, p. 11.

Cependant, la nouvelle configuration médiatique en Tunisie s'est heurtée à plusieurs obstacles, dont la confiance à établir des pratiques journalistiques détachées de toute pression idéologique. Du fait de l'absence d'un cadre juridique réglementant le secteur, la fragilité du métier a été le problème majeur de la période post-14 janvier 2011. Le passage d'un «média d'État» à un «média de service public » a cristallisé un conflit opposant les responsables de la télévision publique aux différents gouvernements de transition, qui n'ont pas réussi à se défaire de la volonté de contrôler les médias ${ }^{18}$.

\footnotetext{
${ }^{18}$ L'expérience à la tête de la rédaction du JT n'était pas évidente pour Said Khezami. Celui-ci s'est trouvé face à des pressions de plusieurs ordres: les manifestants (les sit-inneurs qui veulent
} 


\section{Les partenariats franco-tunisiens au cœur de la reconfiguration de l'espace médiatique}

Dans ce contexte, plusieurs acteurs internationaux (ONG, groupements de bailleurs, diplomaties) ont proposé leur appui aux autorités tunisiennes pour les accompagner dans leur processus de «transition médiatique». Quelques mois après la «révolution » de 2011, le 31 mai, les partenaires historiques ont matérialisé leur offre à travers une «Conférence internationale en faveur de l'audiovisuel tunisien et égyptien ${ }^{19}$ » que I'UNESCO a accueillie à son siège à Paris. Cette dernière a été le chef de file de l'initiative avec le groupe public français France Télévisions. La conférence a également été possible grâce au concours de I'Union européenne de radio-télévision (UER), I'Arab States Broadcasting Union (ASBU), I'Union radiophonique et télévisuelle internationale (URTI) et la British Broadcasting Corporation (BBC), qui a organisé la réunion préparatoire à Londres.

Les organisateurs avaient fixé officiellement un double objectif à la conférence: sensibiliser la communauté des donateurs aux besoins actuels et potentiels de l'audiovisuel de la Tunisie et de l'Égypte; élaborer par la suite une stratégie d'assistance pour le développement d'un audiovisuel public indépendant dans les deux pays. Une expression est revenue à plusieurs reprises dans les interventions des organisateurs de la journée: "les pays arabes nous donnent aujourd'hui une leçon en termes de démocratie». En effet, les différents opérateurs ont déclaré vouloir donner une nouvelle forme à la coopération, c'est-à-dire qu'elle soit bâtie sur l'échange, la réciprocité et non sur un principe de sens unique. Cette stratégie n'est pas nouvelle, comme l'a montré au début des années 2000 la politiste Claire Visier, résumant ainsi les caractéristiques de la coopération française: «Considérer l'autre comme un partenaire à part entière, le reconnaître comme son égal; partager avec l'autre et non lui donner sans retour, ce qui implique sa prise en compte au-delà des différences et des écarts qui peuvent exister (...) : ainsi, l'action est envisagée à partir du partage, qu'il soit intellectuel (la coopération c'est le partage des expériences, des pratiques et des savoirs accumulés), matériel, voire financier.» (Visier, 2003, p. 90)

\footnotetext{
être filmés), la pression à l'échelle internationale autour de l'image de la Tunisie à l'étranger, le gouvernement en place qui souhaitait contrôler l'information.

${ }^{19}$ Dans le cadre de notre travail de terrain et d'observation, nous avons assisté à cette conférence et effectué des entretiens avec quelques acteurs impliqués dans la mise en œuvre de l'initiative. La conférence a également été l'occasion de prendre contact avec les professionnels des médias et des acteurs clés de la coopération audiovisuelle internationale avec la Tunisie.
} 
En attendant de renouer avec une présence plus forte dans le paysage audiovisuel tunisien via la diffusion ${ }^{20}$, la diplomatie française a choisi de renforcer la coopération dans le domaine des médias, en se positionnant en tant que partenaire privilégié des pays du Sud, particulièrement ceux touchés par un vent de changement politique comme la Tunisie. Canal France International $(\mathrm{CFI})^{21}$ est désormais l'opérateur privilégié du ministère des Affaires étrangères ${ }^{22}$ en la matière. Ses missions officielles consistent à "accompagner les chaînes de télévision et les nouveaux médias dans leur professionnalisation et encourager l'autonomisation de leur fonctionnement (dans les secteurs de la gestion, du management, etc. ${ }^{23} \%$.

\section{Illustration 2}

\section{Infographie sur la politique d'aide au développement des médias à travers l'action de CFI en 2011}

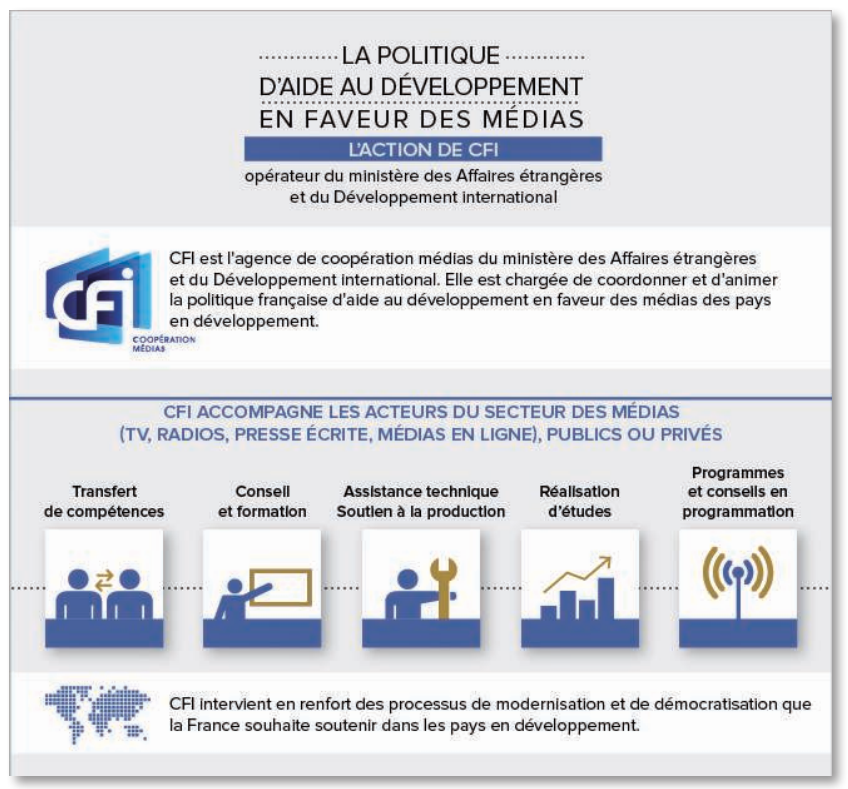

Source: CFI.

\footnotetext{
${ }^{20}$ La deuxième chaîne française France 2, anciennement Antenne 2, était diffusée sur le réseau hertzien en Tunisie à partir de la fin des années 1980. L'expérience a été interrompue en 1999 dans la vague de verrouillage entreprise par le régime de Ben Ali.

${ }^{21}$ Sur ce point, on renvoie au chapitre de Simon Mangon dans cet ouvrage.

${ }^{22} \mathrm{Cf}$. http://www.diplomatie.gouv.fr/fr/politique-etrangere-de-la-france/diplomatie-culturelle/ les-domaines-d-action-de-la-diplomatie-culturelle/article/medias-et-audiovisuel

23 lbid.
} 


\section{La genèse de l'intervention française en faveur des médias tunisiens au lendemain de la « révolution » de janvier 2011}

Composante la plus ancienne de la politique culturelle extérieure de la France, la promotion de la culture française à l'étranger relève principalement du ministère des Affaires étrangères et couvre un champ très vaste: renforcement de l'usage de la langue française, éducation et échanges universitaires, coopération scientifique et technique, échanges artistiques, livre, promotion de l'audiovisuel français (cinéma, télévision, radio). La politique définie par le ministère des Affaires étrangères est mise en œuvre par le réseau de coopération et d'action culturelle à l'étranger, composé des services culturels des ambassades de France et des consulats, des centres culturels et instituts français à l'étranger (environ 150), de 25 centres de recherche en sciences sociales et humaines, ainsi que des Alliances françaises (un millier environ, mais d'importance inégale).

Deux mois après le départ de Ben Ali et le changement de gouvernement en Tunisie, CFI a entamé une mission d'évaluation menée conjointement avec I'Union européenne de Radiodiffusion (UER) en présence d'Hélène Delmas, attachée audiovisuelle régionale de $\mathrm{CFI}$, afin de rencontrer les principaux acteurs du secteur médiatique et d'évaluer la nature de leurs besoins et de leurs priorités, notamment en matière de formation. Cette mission intervient après une «crise» entre les deux pays, du fait de la «position française» sur les mouvements de contestations en Tunisie et de ce que l'opposition à l'Assemblée nationale a qualifié d'« extrême retenue de la réaction française face à la répression en Tunisie». En effet, Michèle Alliot-Marie, la ministre des Affaires étrangères de l'époque, avait proposé à Ben Ali, la veille de sa chute, le savoir-faire français pour prêter main forte à la police tunisienne en vue de «régler les situations sécuritaires». Dès lors, pour Éric Soulier ${ }^{24}$, alors directeur de CFI pour la région Méditerranée-Asie, l'accompagnement des médias tunisiens a pu surtout se développer après ce qu'il appelle ce «petit bug».

«Il y a eu des petits bugs au début, et c'est déplorable, mais même s'il n'y en a pas eu, la résultante ne dépend pas de l'incompréhension ni de la mauvaise analyse qui a été faite. Je pense qu'aujourd'hui le naturel a repris le dessus, et il est important d'accompagner le changement en Tunisie; ça serait une erreur assez importante qu'on ne puisse pas le faire. C'est important aussi bien pour la France que pour la Tunisie de continuer ce rapport d'amitié et que des agences comme nous qui interviennent sur un secteur bien défini

\footnotetext{
${ }^{24}$ Éric Soulier quitte son poste de directeur Méditerranée-Asie chez CFI au mois d'août 2012 pour ses nouvelles fonctions de Conseiller de coopération et d'action culturelle (COCAC) à I'ambassade de France en Australie.
} 
travaillent avec la télévision, les bloggeurs, etc. Je pense que ça serait une faute grave si nous ne le faisions pas.» (Entretien, avril 2011, Paris)

L'état des lieux, qui a été dressé lors de la mission de diagnostic, a révélé que les médias tunisiens, qu'ils soient publics ou privés, en dépit de l'impératif de changer leur image ternie par l'ancien régime, étaient très écoutés et regardés au lendemain du 14 janvier 2011. Les discours des représentants des institutions tunisiennes donnent une place de choix aux médias dans le renforcement de la "citoyenneté", notamment celle des jeunes qui apparaissent avec le public des régions et les internautes comme des «publics-clés» du nouveau paysage médiatique en Tunisie. Un programme en deux phases a été proposé en adéquation avec la demande des autorités nationales, comme l'explique Hélène Delmas, c'est-à-dire un «soutien à la transition démocratique dans le secteur des médias " pour la période pré-électorale (élections de l'Assemblée nationale constituante de 2011) et une "pérennisation à travers des actions structurantes» (fin 2011-2012) (entretien, mars 2011, Tunis).

CFI a effectué cette mission sous l'égide de l'ambassade de France, dans le cadre de sa mission au service des opérateurs audiovisuels français qui interviennent sur financement public au profit d'«opérateurs du Sud». Les orientations ébauchées ont également été confirmées par des missions ponctuelles menées par des représentants de l'Audiovisuel extérieur de la France ${ }^{25}$ (AEF) ou de France Télévisions qui ont pu faire part, comme l'attestent nos interviewés travaillant à $\mathrm{CFI}$, des évolutions que connaît la Tunisie ainsi que des opportunités et demandes nouvelles.

\section{État des lieux de CFI de la scène médiatique tunisienne en mars 2011}

- 2 chaînes de télévision publiques.

- 3 chaînes de télévision privée (Hannibal, Nessma, Ettounsseya).

- 12 radios (4 réseaux publics).

- 3 principaux journaux de presse quotidienne.

- Plusieurs journaux électroniques récents à dominante économique.

- Médias essentiellement arabophones.

- Sondages et mesures d'audience peu développés et très contestés.

$\rightarrow$ Pour 10,6 millions d'habitants en 2011, 2,4 millions de foyers son équipés à $90 \%$ en télévision et $50 \%$ en internet.

\footnotetext{
${ }^{25}$ Audiovisuel extérieur de la France (AEF) est l'appellation d'usage donnée de 2008 à 2013 à la société nationale de programme, créée le 4 avril 2008 pour superviser et coordonner les activités des radios et télévisions publiques détenues par l'État français et ayant une diffusion internationale. L'AEF est baptisée aujourd'hui France Médias Monde.
} 
Cette intervention forte de la France s'inscrit dans le cadre de la traditionnelle coopération étatique, "fondée sur la notion d'aide au développement qui suppose d'aider les pays les moins développés à tirer parti des techniques modernes en leur permettant de se procurer le personnel, le matériel et les fournitures techniques nécessaires au développement économique» (Visier, 2003, p. 146). Ainsi, le projet de coopération dans le secteur audiovisuel de l'acteur étatique français en Tunisie a été orienté vers la restructuration du champ télévisuel, essentiellement en y mettant les moyens humains et financiers.

À partir du mois d'avril 2011, CFI a positionné une correspondante à Tunis, chargée de coordonner les contacts et la mise en place logistique des principales interventions dans le secteur. Hélène Delmas, coordinatrice médias pour CFI basée à I'Institut français et devenue ultérieurement attachée audiovisuelle régionale (Tunis, Maroc et Algérie), a été la principale interlocutrice des acteurs du secteur médiatique tunisien, étant chargée de trois grandes missions: I'accompagnement des médias publics, la coordination des opérateurs de la coopération, qu'ils soient français ou internationaux, et l'appui au développement des nouveaux médias.

\section{L'accompagnement français visant à perfectionner la production de l'information dans la télévision publique}

Les axes de l'intervention de CFI à partir de 2011 ont été arrêtés à la lumière du diagnostic établi, comme l'explique Hélène Delmas, qui y a participé.

"D'après mon observation du terrain, on est dans un constat de professionnalisme assez bas, c'est plus bas que ce que l'on croyait. On n'a pas à faire à des gens qui ont été muselés et qui vont changer du jour au lendemain ce qu'ils écrivent; il y a surtout un manque de professionnalisme qui s'explique par des années où le recrutement à I'IPSI a été laminé, où la façon de travailler a été peu rigoureuse. Ce n'est pas la volonté qui manque, mais plutôt le résultat de vingt-trois ans où on a laminé cette profession. CFI est là pour accompagner dans cette phase d'urgence, on intervient principalement à la télévision sur laquelle on fait un constat de niveau moins bon qu'à la radio, qui a pour sa part gardé sa culture du direct alors que la télé l'a complètement perdue. Cela engendre un manque de professionnalisme plus profond, la peur, les réflexes à recréer.» (Entretien, mars 2011, Tunis) 
La journaliste de télévision et présentatrice du JT en langue française Samira Mahdaoui ${ }^{26}$ partage ce constat sur le perfectionnement nécessaire des pratiques journalistiques.

«L'apport de l'accompagnement proposé par CFI serait d'orienter les journalistes vers plus de rigueur. Tout est à refaire, il faut acquérir de nouvelles habitudes de travail; informer juste et en toute honnêteté, tout en sachant que la phase actuelle exige que l'on revienne aux bonnes règles journalistiques, et se dépoussiérer de plus de deux décennies de journalisme propagandiste. En bref, faire tout bonnement son métier n'est pas une tâche aisée.» (Entretien, août 2011, Tunis)

Suite à un consensus entre CFI et la télévision publique nationale, le choix a été fait de concentrer les efforts sur l'information, à travers notamment la modernisation et le perfectionnement du JT de la Watanya 1 aussi bien sur le fond (le renforcement des compétences des journalistes) que sur la forme (la refonte de l'aspect graphique). Une série de missions de conseil et de formation ont été coordonnées en ce sens par l'opérateur français.

\section{L'offre de formation, une montée en compétence pour les journalistes tunisiens et une opportunité financière pour les organes de l'AEF}

La formation assurée conjointement par CFI et France 24 en vue de la préparation des élections a marqué le début de l'intervention française en Tunisie au lendemain du 14 janvier 2011. Assurée par les formateurs de France 24, une session a eu lieu au sein même de la chaîne de télévision tunisienne du 11 au 29 avril. La mission a été menée par trois formateurs: le journaliste-présentateur francophone Antoine Cormery et directeur de

\footnotetext{
${ }^{26}$ Le JT en langue française a été suspendu depuis janvier 2011 et n'a toujours pas été remis à l'antenne. La journaliste considère son journal comme «une fenêtre ouverte sur la francophonie» qui revêt une importance dans la perspective partenariale dans le domaine audiovisuel qui mérite que l'on s'attarde sur ses enjeux culturels politiques et sociaux. Malgré le processus engagé au lendemain de 2011 en vue de sa refonte et sa remise à l'antenne, le JT en langue française est toujours en stand-by. Faute d'équipes, de moyens mais surtout parce qu'il ne s'agit pas d'un dossier prioritaire pour les directeurs avec toutes les urgences et les interventions à faire sur le JT en langue arabe. Hélène Delmas, en poste d'attachée audiovisuelle régionale, regrette ce processus inachevé: «Nous avons assuré la formation des équipes mais les moyens n'étaient pas tous là, il manquait des recrutements, et avec tous les chantiers ce dossier n'était pas vraiment prioritaire. En tout cas, moi j'y travaille encore et je ne perds pas espoir, mais voilà, il faut du temps. Compte tenu des 600000 Tunisiens en France et qui ne sont pas tous arabophones, le JT en langue française s'impose en Tunisie, et puis il peut constituer une ouverture sur l'Afrique francophone, le continent africain représente $6 \%$ de croissance annuelle. La langue française est un atout pour la Tunisie qui est facile à mettre en œuvre.» (Entretien, mars 2011)
} 
I'Académie France $24^{27}$, accompagné de deux collègues de la rédaction arabophone, Taoufik Mjaïed, un rédacteur en chef, et Adel Gastel, un grand reporter. Pendant les trois semaines de formation au sein de la rédaction News, le travail quotidien aux côtés des journalistes avait pour but de répondre aux attentes les plus urgentes: modes d'écriture de sujets d'information politique, réalisation d'interviews, préparation des questions, suivi de l'interview. Cette mission a été assurée par le département de formation de France 24 qui, suite à l'intervention en Tunisie, a mis en place le projet de l'Académie France 24, comme en témoigne Antoine Cormery, son directeur.

«L'Académie France 24 est le département de formation de la chaîne. Elle a été créée à Tunis le 29 avril, à l'issue d'une première mission à la télé tunisienne. Le projet était dans nos esprits depuis un petit moment. Cela fait précisément un an que je travaille dessus, mais il fallait imaginer comment ça pourrait être, quels étaient nos savoir-faire, qu'est-ce qu'on voulait faire de l'académie concrètement. À partir de ce moment, on commençait à se dire que le Maghreb était pour nous une zone intéressante culturellement et géopolitiquement, en termes de voisinage, etc., et puis devant nos yeux ébahis, les révolutions ont commencé dans les pays arabes, et évidemment c'est pour nous une chance et une opportunité.» (Entretien, mai 2011, UNESCO, Paris)

L'initiative de lancer l'Académie France 24 pourrait constituer une réponse à l'idée d'une fusion entre France 24 et Radio France Internationale (RFI), préconisée par un rapport de l'Assemblée nationale française sur I'AEF en 2011 dans lequel France 24 apparaît comme une institution déficitaire. Il est possible que l'Académie ait été pensée comme une issue pour combler les déficits et présenter une réponse aux problèmes financiers de la chaîne. C'est dans cette perspective que les responsables de la chaîne auraient décidé de saisir l'opportunité de cette «demande» de plusieurs pays en pleine restructuration politique pour créer l'Académie et tester ainsi, notamment à partir de la Tunisie, son modèle financier et pédagogique. Disposant de professionnels avec une fine connaissance de l'actualité de cette "révolution», ils ont rapidement mis en œuvre le projet, partant à la rencontre des directeurs de la télévision et de la radio tunisiennes pour proposer le concept: les partenaires français ont pris en charge l'aspect financier par le biais de CFI, essentiellement à travers ses partenaires et donateurs; France 24 a développé le volet pédagogique (formateurs et conception du contenu); la télévision

\footnotetext{
${ }^{27}$ L'Académie France 24, qui a été créée pour l'occasion, a désormais changé de nom pour devenir Académie France Médias Monde, englobant les médias de l'audiovisuel extérieur de la France: France 24, Radio France Internationale, Monte Carlo Doualiya. Cf. http://academie. france24-mcd-rfi.com/fr/category/equipe
} 
tunisienne a assuré de son côté l'hébergement de l'équipe. Antoine Cormery ajoute au sujet de l'Académie France 24:

"La télévision tunisienne veut faire appel à nous parce qu'on a des formateurs arabophones et, pour certains, originaires de cette région-là, donc c'était le profil parfait pour cette formation. Donc de toute façon cette académie devait se lancer, c'est un nouveau département de formation internationale à France 24. L'actualité nous a merveilleusement aidés.» (Entretien, mai 2011, UNESCO, Paris)

Les responsables tunisiens ont répondu favorablement à l'offre de formation de France 24. Ce fut une rencontre, et non pas une simple demande ni offre comme l'explique en entretien le directeur de l'Académie, ses propos étant confirmés par Mokhtar Rassâa, l'ex-directeur de la télévision nationale: "La coopération est venue d'une volonté de chez nous de faire faire des stages à nos journalistes, et ceci a coïncidé avec une offre de France 24, qui a été le premier à satisfaire notre demande via CFI.» (Entretien, mai 2011, Tunis) En effet, cette formation s'inscrit au cœur de la politique de diffusion de la «culture française». Comme le souligne Claire Visier (2003, p. 147), celle-ci relève d'une politique de transfert de savoir-faire, principalement à partir de la formation. Néanmoins, le fait d'avoir des professionnels d'origine tunisienne n'est pas toujours perçu de manière positive par les journalistes bénéficiaires. Le témoignage de Naïma Jouini, journaliste et présentatrice de longue date $\mathrm{du} \mathrm{JT}^{28}$, est révélateur. Elle trouve que le fait d'avoir un ancien collègue comme formateur n'a pas de valeur ajoutée compte tenu de leur background commun. La seule expérience dans un média étranger ne suffit pas, d'après elle, à légitimer sa compétence professionnelle (entretien, février 2012, Tunis).

\section{La formation au sein de la rédaction, pierre angulaire de l'intervention française}

Les sessions de formation assurées par les partenaires français se déroulaient pour l'essentiel au sein même des rédactions. L'objectif premier de ce programme était de contribuer au perfectionnement des journalistes de télévision, comme l'explique Antoine Cormery.

\footnotetext{
${ }^{28}$ Naïma Abdallah Jouini et ses collègues Insaf Ben Moussa et Khadija Soua, présentatrices de la télévision nationale, considérées comme les principales présentatrices du JT de 20 heures d'Al Watanya 1, ont demandé au mois de mars 2012 de ne plus présenter les informations, suite à la pression dont elles faisaient l'objet de la part des sit-inneurs. Cf. https://www.turess. $\mathrm{com} / \mathrm{fr} /$ tunivisions/34204
} 
"L'idée était de faire de l'accompagnement et du coaching de journalistes (il montre des photos pour appuyer son propos). Là tu as Adel Gastel, l'un de nos plus grands reporters qui a couvert la révolution égyptienne, et là, on est dans la Newsroom, la salle de rédaction de la TV tunisienne avec une jeune journaliste. Adel est parti le matin avec elle; ils ont passé la journée sur le terrain à faire du reportage et, le soir, ils sont au montage pour voir comment tout ça s'organise. C'est ça l'essence de notre formation, ce n'est pas du tout théorique, c'est l'accompagnement sur le terrain. Là, c'est Taoufik avec une journaliste et, ici, c'est moi avec un présentateur tunisien. Vous voyez, on travaille avec les journalistes au creux de l'oreille. » (Entretien, mai 2011, UNESCO, Paris)

D'après notre interviewé, il n'y a pas eu d'identification préalable des besoins des bénéficiaires. Les grandes lignes ont été arrêtées avec la direction, puisqu'il s'agissait pour la majorité des sessions d'un accompagnement plus que de formations. C'est sur le terrain que les «besoins» ont émergé, aux formateurs de s'adapter: "L'intervention fut centrée sur des questions de journalistes et non celles d'un théoricien : comment définir un angle? Quels sont les gens à contacter? Les gens à interviewer? Comment mettre en image un reportage? Comment faire sur le terrain?», précise le directeur de I'Académie. Cette intervention au sein-même des rédactions, ainsi présentée par la partie française comme étant inédite et révolutionnaire, se trouve controversée du côté des journalistes tunisiens. L'évaluation faite par les journalistes ayant bénéficié de cet accompagnement sur le terrain n'est pas toujours positive, comme en témoigne la journaliste Malika Jabberi.

«Même si les formateurs sont animés par une bonne volonté, les journalistes bénéficiaires trouvent que les interventions au sein des rédactions constituent plus une perte de temps qu'une valeur ajoutée. Le problème soulevé est relatif au fait que l'accompagnement sur le terrain oblige les journalistes à expliquer chaque étape et à échanger avec le formateur, ce qui a induit un retard dans le travail et la livraison des éléments qui doivent être consultés bien à l'avance par le chef d'édition afin d'être validés pour la diffusion.» (Entretien, mai 2012, Tunis)

Hélène Delmas approuve complètement le choix pratique du programme et n'émet aucun doute sur l'efficience de la méthodologie adoptée. Elle considère à cet effet que les éventuelles difficultés rencontrées par les journalistes à ce sujet renvoient à un problème de compréhension de la méthodologie de l'accompagnement

«Les journalistes ont l'habitude de prendre note, mais je trouve que la force de CFI c'est de savoir s'adapter au contexte et, là, la décision a été de mettre quelqu'un en fil rouge, parce qu'ils n'ont pas l'habitude de faire ça. Je vois que d'autres opérateurs viennent, ils font une mission de reconnaissance. 
Le temps de préparer le financement, ils reviennent dans deux mois et trouvent les choses changées. Donc, sur la base, les journalistes, par manque de professionnalisme pour la majorité, se contentent de formations assises en salle où on raconte. Mais pour les autres, qui ont envie de changer leurs pratiques, ils comprennent notre travail.» (Entretien, mars 2011, Tunis)

Interrogé sur l'impact de ces formations, Zouhaier Latif, journaliste, propriétaire d'une chaîne de télévision et formateur à la $B B C$, ayant assuré certains cycles de formation au profit des journalistes de la Watanya 1 au lendemain de la «révolution», pose le problème du manque d'intérêt de la part des journalistes à l'égard de ces sessions de renforcement des capacités. D'après son expérience, «L'accueil des journalistes n'était pas très enthousiaste vis-à-vis de la formation de manière générale. Au départ, c'était sympathique, les gens venaient, puis on en voyait de moins en moins dans la journée.» (Entretien, septembre 2014, Tunis) Considérant qu'ils disposent déjà d'une expérience conséquente, les journalistes, surtout les plus confirmés, estiment que ces formations ne leur apportent pas de valeur ajoutée.

\section{Conclusion}

En passant en revue les différents points de vue, aussi bien des acteurs français que de ceux des journalistes tunisiens bénéficiaires, force est de constater que les seconds ne fournissent pas une évaluation totalisante des offres de formation. Interrogés sur leur perception de l'intervention française, la plupart des journalistes ne se montrent pas loquaces: certaines réponses sont marquées par des digressions pour éviter d'évoquer les difficultés, d'autres formulent des critiques exacerbées de l'attitude de quelques formateurs.

La manière dont les journalistes dépeignent l'offre de CFI pour le perfectionnement des journalistes tunisiens soulève de fait la question de la langue et la connaissance du terrain qui manque aux formateurs (pour la plupart non arabophones et non Tunisiens) et souligne les limites de l'impact de leurs interventions. La compréhension de la notion de service public s'avère plus longue que prévu: ne dépendrait-elle pas non seulement de la formation, mais aussi et surtout d'un manque d'expérience et de temps?

Face à la demande réelle ou supposée, les différents intervenants ont éprouvé le besoin de regrouper leurs actions, de diversifier la nature de 
la coopération: matériel, préparation d'émissions communes, aide à la rédaction d'une charte déontologique, etc. Un Groupe de partenaires techniques et financiers en appui au secteur médiatique tunisien ${ }^{29}$ a vu le jour, déclarant avoir pour mission ${ }^{30}$ l'harmonisation des actions des donateurs et des opérateurs internationaux, ainsi que l'appropriation de la coordination des politiques d'appui par le pays partenaire, y compris par la «société civile».

L'analyse de la reconfiguration médiatique tunisienne et de l'intervention française qui l'a accompagnée montrerait in fine un renouvellement de la conception de la coopération française audiovisuelle. Ce constat amène à se poser des questions sur la portée de ce changement comme sur ses limites. La politique française ne se réduit bien évidemment pas aux seules actions orientées vers les médias audiovisuels, touchant tout le secteur culturel et médiatique. En effet, le projet de la coopération française apparaît toujours, comme l'a suggéré Claire Visier (Visier, 2003) dans son enquête menée à la fin des années 1990, orienté vers la scène interne des pays maghrébins et de la Tunisie plus précisément dans cette période de l'histoire du pays, dans le but de garder des avantages acquis, de chercher de nouveaux débouchés ou de développer le pays. Ces facteurs comptent assurément, mais on ne peut pas dire qu'ils sont les seuls à entrer en ligne de compte dans une période bouleversée. Comme l'ont analysé plus tard Mohamed Ali Elhaou et Aida Fitouri (2015, p. 39), «ce processus produit un décalage entre, d'un côté, les acteurs internationaux qui agissent souvent en fonction d'idéaux et de calendriers bien précis et délimités dans le temps avec souvent des modèles prêt-à-penser et, de l'autre côté, les acteurs locaux qui agissent selon des logiques centrées sur leurs conditions de travail et sur leurs propres intérêts, sans saisir nécessairement la portée globale et marchande du processus de réformes».

\footnotetext{
${ }^{29}$ Un Groupe média, rassemblant l'ensemble des intervenants au profit des médias tunisiens, a été mis en place par la Délégation Wallonie-Bruxelles en Tunisie le 24 mars 2011 à la demande de la Délégation de I'Union européenne. À partir de décembre 2011, c'est l'ambassade de Suisse en Tunisie qui a repris la supervision des travaux et des réunions du groupe pour une période de dix-huit mois. Depuis le $1^{\text {er }}$ septembre 2013, le pilotage du Groupe des partenaires techniques et financiers en appui au secteur médiatique tunisien est confié au Centre africain de perfectionnement des journalistes et communicateurs (CAPJC).

${ }^{30}$ Ce groupe est baptisé Programme d'appui aux médias en Tunisie, depuis 2015. Financé par I'Union européenne, il est exécuté par le CAPJC. Cf. http://www.mediaup.tn/content/media-up
} 


\section{Bibliographie}

ACHOUR Kallel Myriam, "Mixages de langues et représentations de la diversité : une anthropologie des pratiques linguistiques de Radio Mosaïque FM en Tunisie", Annales de sociologie et d'anthropologie, "Communautés et sociétés, théories de la diversité culturelle et de la mondialisation », Beyrouth, nos 21-22, 2011.

BALANDIER Georges, Le Pouvoir sur scène, Paris, Ed. Balland, coll. Le commerce des idées, 1980.

Ben Abdallah Chirine, Les Nouveaux enjeux de l'information à la télévision tunisienne en situation de transition sociopolitique: la complexité des partenariats France-Tunisie au lendemain de la révolution de 2011, Paris, thèse en sociologie, Sorbonne Paris et Cité-Université de Tunis Al-Manar, 2016.

BEN ABDALLAH Chirine, "Les nouveaux usages sociaux des produits artistiques à l'ère des TIC: le "piratage" des œuvres artistiques en Tunisie», dans Les Nouvelles sociabilités du Net en Méditerranée, sous la dir. de S. Najar, Paris, IRMC-Karthala, 2012.

BRAS Jean-Philippe, CHOUIKHA Larbi, "Introduction", dans Médias et technologies de communication au Maghreb et en Méditerranée: mondialisation, redéploiements et "arts de faire», sous la dir. de J-Ph. Bras et L. Chouikha, Tunis, Institut de recherche sur le Maghreb contemporain, 2002. DOI: https://doi-org.inshs.bib.cnrs.fr/10.4000/books.irmc. 218

BRAS Jean-Philippe, "Ordre public, politiques publiques et Internet en Tunisie», dans Médias et technologies de communication au Maghreb et en Méditerranée: mondialisation, redéploiements et "arts de faire», idem. DOI: https://doi.org/10.4000/books.irmc.224

CABEDOCHE Bertrand, "La construction de l'étrangéité, enjeu du projet de chaîne française d'information internationale», Cahiers du journalisme, $n^{\circ} 14,2005$. http://www.cahiersdujournalisme.net/pdf/14/18_Cabedoche. pdf

CABEDOCHE Bertrand, "Télévisions transnationales et représentation de l'altérité », dans IPSI et la Fondation Konrad Adenauer, Cahiers du journalisme, $\mathrm{n}^{\circ} 17,2007$. https://www.cahiersdujournalisme.net/ pdf/17/21_Cabedoche.pdf

CHOUIKHA Larbi, «Fondements et situation de la liberté de l'information en Tunisie: essai d'analyse», dans L'Information au Maghreb, sous la dir. de W.S. Freund, Tunis, Cérès, 1992. 
CHOUIKHA Larbi, «L'audiovisuel en Tunisie: une libéralisation fondue dans le moule étatique», L'Année du Maghreb, nII, 2005-2006, mis en ligne le 8 juillet 2010. DOI: https://doi.org/10.4000/anneemaghreb.165

DeBBASCH Charl es, La République tunisienne, Paris, Librairie générale de droit et de jurisprudence (LGDJ), 1962.

ELHAOU Mohamed Ali, FITOURI Aida, "La profession journalistique après la "révolution du jasmin" sous l'emprise de la modernisation", Les Enjeux de l'information et de la communication, $\mathrm{n}^{\circ}$ 1, 2015. DOI: https://doi. org/10.3917/enic.018.0039

FERJANI Riadh, «Antenne 2/France 2 comme enjeu social et politique en Tunisie », Les Enjeux de l'information et de la communication, n 1, 2001. DOI : https://doi.org/10.3917/enic.001.0034

GUAAYBESS Tourya, "Les trois âges du regard occidental sur l'opinion publique arabe», dans Médias et opinion publique, sous la dir. d'A. Mercier, Paris, CNRS éditions, 2012, coll. Les essentiels d'Hermes. DOI: https://doi. org/10.4000/books.editionscnrs.19051

HADDAD Khaled, Bourguiba et les médias: dialectique du pouvoir et de la propagande, Tunis, Impressions Carthage, 2008.

INSTITUT PANOS PARIS ET OBSERVATOIRE MÉDITERRANÉEN DE LA COMMUNICATION, Médias et régimes autoritaires, Paris, 2012. http://www.omec.es/Documentos/ PSB_Brochure_160x240_FR-bd.pdf

KHELIL Hédi, "Habib Bourguiba. Les trois nouveaux enjeux: la télévision, l'ordinateur et la monnaie », Annuaire de l'Afrique du Nord, n²3, 1986.

$\mathrm{KOCH}$ Olivier, "La (re-)professionnalisation du journalisme tunisien dans la période transitionnelle: le rôle des acteurs extranationaux», dans La Circulation des productions culturelles: cinémas, informations et séries télévisées dans les mondes arabes et musulmans, sous la dir. de D. Marchetti, Istanbul-Rabat, Editions du Centre Jacques Berque, 2017. DOI : https://doi.org/10.4000/books.cjb.1219

KRIDISS Noureddine, Communication et innovation, Paris, L'Harmattan, 2008.

LECOMTE Romain, «Internet et la reconfiguration de l'espace public tunisien: le rôle de la diaspora», vol. 3, n 1-2, 2009. DOI: https://doi.org/10.4000/ ticetsociete.702

LERNER Daniel, The passing of traditional society: Modernizing the Middle East, Glencoe, The Free Press, 1958. 
LeTEINTURIER Christine, «Lectures: Florence Millerand, Serge Proulx et Julien Rueff (dir.), Web social: mutation de la communication", Communication, vol. 30, n 1, 2012. DOI: https://doi.org/10.4000/communication.3053

LÉVy Pierre, Cyberdémocratie: essai de philosophie politique, Paris, Éd. Odile Jacob, 2002.

TOUATI Zeineb, «Évolution du traitement médiatique des reconfigurations identitaires: entre spécificités des médias et impératifs de la transition démocratique, cas de la Tunisie post-2011 », French Journal For Media Research, $\mathrm{n}^{\circ} 8$, 2017. https://frenchjournalformediaresearch.com:443/ lodel-1.0/main/index.php?id=1407

VISIER Claire, L'État et la coopération, la fin d'un monopole: l'action culturelle française au Maghreb, Paris, L'Harmattan, 2003.

\section{Sources}

INSTITUT DE SONDAGE ET DE TRAITEMENT DE L'INFORMATION STATISTIQUE (ISTIS), "Sondage d'opinion sur la situation politique et l'impact de la révolution sur les média presse», Tunis, avril 2011. https://fr.slideshare.net/ikays/ rapport-situationpolitiquemediapresse-7765803

GoNZALES Paule, «France 24 en arabe: un pari réussi au Maghreb ». http:// blog.lefigaro.fr/gonzales/2011/03/elle-simpose-moins-dun-an.html

PuCHOT Pierre, «Journaux, Université... En Tunisie, on ferme !», Mediapart, 29 janvier 2010. https://blogs.mediapart.fr/pierre-puchot/blog/290110/ journaux-universite-en-tunisie-ferme 


\title{
Chapitre 8 \\ Au chevet du journalisme tunisien? \\ Fabrique de l'aide étrangère, effets sur la profession et stratégies de réappropriation
}

\author{
Simon Mangon
}

Le "développement médias ${ }^{1}$ » est une branche spécifique des politiques de développement dont l'objectif est de soutenir le secteur médiatique d'un pays par une aide extérieure. L'appui aux médias dits "bénéficiaires», qu'il soit financier, matériel ou de type «renforcement des capacités ${ }^{2} »$, est censé "promouvoir l'indépendance, la pluralité et le professionnalisme» (Scott, 2014). Le secteur est investi par un ensemble d'acteurs, allant de grands médias publics européens aux ONG en passant par des fondations privées ou des organisations gouvernementales. Ces structures majoritairement américaines ou européennes mettent en place des programmes d'appui au journalisme dans des pays dits "du Sud», notamment en contexte de «transition démocratique ${ }^{3}$ ». La Tunisie, «bon élève» des bailleurs de fonds internationaux depuis plusieurs décennies (Hibou, 1999), n'a pas échappé à ces dispositifs. Depuis le moment révolutionnaire ${ }^{4}$ tunisien de 2011, une

\footnotetext{
${ }^{1}$ Les acteurs parlent aussi d'«aide», de «coopération» ou d'«appui aux médias ». Nous faisons le choix d'utiliser des guillemets pour prendre de la distance avec ces expressions qui invisibilisent les rapports de force et les logiques de domination pourtant à l'œuvre.

${ }^{2}$ Cette expression renvoie aux activités de formation des membres d'un média par des intervenants extérieurs.

${ }^{3}$ Le rôle des médias en période de «transition » fait l'objet de multiples travaux de la part de chercheurs ou d'organisations internationales. Pour une lecture critique, voir Mattelart, 2020.

${ }^{4}$ Nous privilégions l'expression «moment» ou «situation» révolutionnaire (Revue française de science politique, 2012) plutôt que "révolution» pour se démarquer de l'idée d'une vague unifiée d'insurrections motivées par les mêmes causes, et analyser de manière dynamique ces situations politiques, en se penchant sur les interactions entre acteurs.
} 
trentaine d'organisations étrangères spécialisées dans l'appui aux médias multiplient les programmes de formation, les colloques et les subventions. Loin d'être un appui technique neutre, le "développement médias» revêt des enjeux politiques importants. Canal France International (CFI), I'agence française de développement médias, fait partie de ces structures qui ont soutenu des nouveaux médias "citoyens » ou "associatifs » après 2011 afin de renforcer le "pluralisme» et le rôle de la «jeunesse tunisienne ${ }^{5}$ » dans le secteur. La définition de cette stratégie répond à des intérêts politiques plus larges, la structure étant sous la tutelle du ministère français des Affaires étrangères et de la filiale du groupe France Médias Monde, l'acteur principal de l'audiovisuel extérieur français ${ }^{6}$. Cette intervention étrangère dans le champ journalistique tunisien est, selon ses promoteurs, un vecteur de «réforme» d'un secteur marqué par plusieurs décennies de contrôle politique. La profession a été réduite au rôle de porte-parole du pouvoir par les présidents Bourguiba et Ben Ali, qui avaient en commun de réprimer les voix contestataires et d'utiliser la presse pour présenter une image «moderne» de la Tunisie à l'extérieur.

Si le moment révolutionnaire de 2011-2012 a bien représenté un tournant en ouvrant largement le secteur à de nouveaux acteurs (Klaus et Koch, 2017), des logiques héritées de l'ancien régime persistent toujours (Klaus, 2017), confortant l'emprise des pouvoirs politiques et économiques sur la profession. Pour les médias qui craignent cette "double dépendance» (Champagne, 1995) à la loi du profit et à l'affiliation politique, l'intensification du soutien d'acteurs étrangers à partir de 2011 est une aubaine pour se prémunir de l'influence de pouvoirs locaux. II en va ainsi du média numérique Inkyfada ${ }^{7}$, créé en 2013 et considéré comme le principal média tunisien d'investigation aux yeux des acteurs étrangers. Né de la rencontre entre d'anciens cyber-activistes militants luttant contre le régime de Ben Ali et d'une nouvelle génération de journalistes, le discours du journal fait écho aux attentes des bailleurs étrangers. Le média Inkyfada est loin d'être

\footnotetext{
${ }^{5}$ Entretien avec un responsable de projet en Tunisie, janvier 2020.

${ }^{6}$ L'audiovisuel extérieur de la France, aujourd'hui appelé «France Médias Monde», est un groupe qui coordonne les activités des radios et télévisions détenues par l'État français ayant une diffusion internationale (Radio France Internationale, France 24, Radio Monte Carlo Doualiya et Canal France International).

${ }^{7}$ Mélange de l'arabe intifada (إنتفاضة ou «soulèvement») et de l'anglais ink (encre), le projet encourage un «soulèvement par l'encre».
} 
le seul à recevoir un soutien d'organisations étrangères ${ }^{8}$, mais ces médias dits «non lucratifs» ou «indépendants» sont ceux qui entretiennent les relations les plus denses avec des organisations non tunisiennes. Leur modèle économique repose en grande partie sur ces aides extérieures, ils font partie de réseaux internationaux de médias, les journalistes ont souvent été formés à l'étranger ou par des médias étrangers.

Ce chapitre se propose d'analyser les effets de cette "aide» extérieure sur la profession journalistique en Tunisie en prenant Inkyfada comme cas d'étude. Il s'agira donc de retracer le processus de définition d'un modèle journalistique à l'étranger (en France, à travers le cas de CFI), sa diffusion par des programmes d'appui aux médias tunisiens, puis sa réception par les journalistes dits «bénéficiaires». En réfutant le mythe d'une supposée influence étrangère sur la ligne éditoriale du média, nous faisons plutôt I'hypothèse que ces relations avec des organisations extérieures au champ journalistique contribuent à redéfinir la vision du "bon journalisme» en Tunisie. Ainsi, le journaliste doit être capable d'assurer un ensemble d'activités non éditoriales (recherche de financements, montage de projets, formation, communication) et être en mesure de prouver son «impact» aux bailleurs de fonds. Si «l'aide» étrangère aux médias n'influence pas directement leur ligne éditoriale, elle contribue à transformer la sociologie de leurs membres, les pratiques professionnelles et la conception même qu'ils ont de leur métier. Une autre hypothèse est que la diffusion de ce modèle journalistique ne se fait pas de manière mécanique, mais qu'elle fait plutôt l'objet de stratégies de réappropriation de la part des journalistes qui traduisent les préoccupations des bailleurs selon leurs besoins et conservent ainsi une forme d'autonomie.

Ce travail est à la croisée de la sociologie des médias et de la sociologie des politiques de développement. L'enquête de terrain a été réalisée dans le cadre d'un doctorat à I'IEP d'Aix-en-Provence sur les effets des politiques de «développement médias» en Tunisie et en Jordanie. Les données empiriques ont été récoltées à Tunis et à Paris, elles sont issues d'entretiens biographiques avec les journalistes et les professionnels de "l'aide», d'observations du travail journalistique et d'analyse de documents internes aux organisations.

\footnotetext{
${ }^{8}$ La spécificité de l'appui international aux médias en Tunisie est qu'il concerne une grande partie du secteur: médias publics, privés et associatifs, organisations professionnelles, instances indépendantes, institutions publiques.
} 


\section{Description d'un média «bon élève » de l'aide internationale}

En quelques années, le journal en ligne Inkyfada est devenu, aux yeux des acteurs internationaux du «développement médias», le «bon élève» tunisien capable de répondre aux critères d'excellence professionnelle. Cela s'explique d'abord par sa ligne éditoriale, orientée sur les thématiques prioritaires des bailleurs de fonds internationaux comme les droits de l'homme, la situation des minorités, l'égalité des sexes ou l'écologie. De plus, Inkyfada revendique développer le journalisme d'investigation, une pratique professionnelle à laquelle les organisations étrangères attribuent un ensemble de vertus allant du rôle de "chien de garde» de la démocratie à la «responsabilisation des élites politiques». Enfin, le format du contenu produit, qui allie innovation numérique (infographie, data-journalisme), goût pour l'esthétique (photos de grande qualité, dessins) et valorisation de l'enquête (extraits d'entretiens, récits de terrain), permet de visualiser «l'impact» exigé par les bailleurs de fonds. Au-delà de ces principales caractéristiques, il semble important d'aller plus en profondeur dans la description du journal pour comprendre en quoi il répond aux attentes des acteurs de «l'aide» et avant d'analyser ce que ce soutien extérieur fait à la structure. Dans cette première partie, il s'agira d'étudier la trajectoire historique de Inkyfada, les fondements de sa ligne éditoriale, les enjeux linguistiques qui le traversent, la sociologie de ses membres et, enfin, sa position dans le champ journalistique.

La création d'Inkyfada à Tunis en 2013 doit être analysée au prisme des récents changements du champ journalistique national. Ce genre journalistique s'est d'abord défini en opposition au «journalisme de complaisance " (Chouikha, 2015) caractéristique de l'étatisation de l'information organisée sous la présidence de Habib Bourguiba (1957-1987), puis de Zine al Abidine Ben Ali (1987-2011). De l'indépendance du pays en 1956 au moment révolutionnaire de $2011^{9}$, les deux présidents tunisiens ont fait de la presse le porte-parole du pouvoir politique par la répression et la marginalisation de toute voix dissidente. Cette stratégie politique visait à discipliner les pratiques journalistiques et les faire tendre vers la production d'une image "positive» du pays à l'étranger, un élément fondamental du système économique et politique de la Tunisie (Hibou, 1999). La fragilisation

\footnotetext{
${ }^{9}$ Il y a bien eu quelques périodes « d'ouvertures relatives» (Chouikha, 2015), notamment entre 1977 et 1985, lors dudit «printemps médiatique», mais le pouvoir politique a toujours gardé la main sur le secteur.
} 
du monopole étatique sur le secteur de la presse a commencé au début des années 2000 par des contestations émanant de la profession ${ }^{10}$, I'apparition des premiers médias privés et l'émergence de la cyber-dissidence (Lecomte, 2013). L'héritage du journal Inkyfada réside dans la contestation en ligne du pouvoir de Ben Ali, puisqu'une majorité des fondateurs étaient membres de Nawaat (en arabe: نواة, ou "noyau»), une plateforme de blogueurs citoyens particulièrement actifs dans la lutte contre l'ancien régime. Ce collectif de cyber-activistes avait notamment publié les vidéos des premières manifestations à Sidi Bouzid et les images violentes à l'hôpital de Kasserine qui confirmaient la sanglante répression organisée par Ben Ali mais passée sous silence par les médias d'État. Le blog collectif est animé par des Tunisiens de la diaspora qui vont s'allier à des activistes installés dans le pays pour couvrir l'actualité politique et ouvrir le débat. Le parcours de l'ancienne rédactrice en chef de Nawaat, qui sera ensuite co-fondatrice $\mathrm{d}^{\prime}$ Inkyfada, est révélateur de cette progressive professionnalisation. Issue d'une famille de banquiers pro-Ben Ali, elle arrête ses études de finances et se tourne vers les lettres modernes avant d'enseigner quelques années. En 2008, au moment des grèves du bassin minier de Gafsa ${ }^{11}$, elle s'insurge contre la censure et la répression organisées par le pouvoir politique. Elle décide de briser le silence et commente l'actualité politique sous la forme de billets publiés anonymement sur Nawaat. Très active sur Facebook, elle intègre le réseau des militants, avocats et blogueurs installés en Tunisie et lance le «musée virtuel de la censure» pour regrouper toutes les initiatives bloquées par le régime de Ben Ali. Devenue rédactrice en chef après 2011, elle raconte la transformation progressive de la plateforme Nawaat.

"On a toujours eu une vraie dynamique militante, notre point commun, c'était les idéaux de liberté d'expression, de transparence, de droits humains, et pas vraiment le journalisme en fait (...). C'est à partir de 2011 que j'ai dû apprendre le métier, ce n'était plus simplement du blogging, il fallait bien faire la distinction entre un article d'opinion et une dépêche (...). J'ai commencé à apprendre seule sur internet, et puis j'ai été accompagnée par les journalistes de formation qui sont arrivés ensuite.»

\footnotetext{
10 On pense notamment à la grève de la faim particulièrement médiatisée du journaliste Taoufik Ben Brik en 2000, aux mobilisations du syndicat national des journalistes ou, audelà de la profession, au mouvement du 18 octobre de contestation de la censure lancé par des opposants au régime de Ben Ali en parallèle du Sommet international sur la société de l'information accueilli à Tunis en 2005.

${ }^{11}$ Vaste mouvement social qui touche la région minière de Gafsa au sud de la Tunisie pendant six mois en 2008. Première grande mobilisation depuis les années 1980, cet événement est considéré comme le début du processus qui va mener au mouvement révolutionnaire de 2011.
} 
En effet, le journal acquiert progressivement une reconnaissance internationale par l'action de jeunes journalistes diplômés et dotés de capitaux sociaux et culturels, comme cela peut également s'observer dans d'autres sphères du militantisme tunisien post-2011 (Ben Mami, Gobe, 2021). Cette nouvelle génération issue d'écoles de journalisme apporte les «codes professionnels» qui manquaient à la plateforme de blogueurs. L'équipe du journal se scinde progressivement en deux parties, un groupe militant qui veut conserver le format du blog participatif, et un second groupe qui veut professionnaliser la structure et constituer une véritable équipe de rédaction. L'une des journalistes de formation arrivée à Nawaat en 2012 décrit ce décalage sur les questions de professionnalisation.

«J'étais l'une des seules à avoir fait une école de journalisme, et j'ai vite senti qu'une grande partie de l'équipe manquait des bases du métier: les questions d'éthique, le recoupage des faits, la nécessité d'avoir plusieurs sources (...). Toute une partie de la rédaction voulait faire du journalisme de qualité, mais cela tiraillait avec l'aspect militant, qui était l'ADN de Nawaat.»

Inkyfada est le résultat de cette opposition entre deux visions du journalisme au sein de Nawaat. Les membres partisans d'un journalisme «professionnel» décident de quitter le groupe et de lancer un nouveau média formé autour d'une équipe de rédaction plutôt que sur des contributions, afin de produire un "journalisme militant mais rigoureux ${ }^{12}$ ». Cette trajectoire historique d'un journal héritier de la lutte contre le régime de Ben Ali explique également les fondements de sa ligne éditoriale. L'identité du média s'est construite sur l'ambition de représenter les "sansvoix», de donner un rôle politique au journaliste par l'investigation et de promouvoir des idéaux dits «progressistes».

Les expressions utilisées par les membres d'Inkyfada pour présenter la structure convergent vers l'idée d'un tiers-secteur, c'est-à-dire une voie alternative à l'opposition traditionnelle entre médias publics et médias privés. Les journalistes parlent de média "indépendant», «à but non lucratif», "associatif» ou «de la société civile» pour caractériser cette ambition d'une information produite pour l'intérêt général, plutôt qu'au service d'intérêts économiques ou partisans. Cela se traduit concrètement dans le style d'écriture des journalistes qui veulent rendre justice aux «gens ordinaires » souvent invoqués dans les articles d'Inkyfada, présentés comme les victimes d'un système politique qui ne les représente pas. La plupart des

${ }^{12}$ Entretien avec une journaliste, Tunis, octobre 2020. 
enquêtes s'appuient sur des entretiens avec des ouvriers, des agriculteurs, des migrants ou des minorités sexuelles. Les journalistes donnent une voix aux «sans voix» (Ferron, 2012) et militent pour une meilleure représentativité de ces franges de la population dans le débat public, à l'image de certains médias privés dans les années 2000 en Égypte (Benaziz, 2015). On pourrait supposer que les revendications populaires de 2011 pour une plus grande «justice sociale» (Hmed, 2016) ont eu un écho dans le champ journalistique, via la promotion de ce genre journalistique qui se fait le défenseur des classes populaires. L'environnement social dans lequel s'inscrit l'équipe d'Inkyfada va dans ce sens puisque les journalistes collaborent fréquemment avec des associations locales ou internationales beaucoup plus qu'avec des médias tunisiens. Ainsi, plusieurs enquêtes ont été réalisées en partenariat avec Amnesty International ou Avocats sans frontières, des coopérations sur des questions juridiques sont fréquentes avec l'association tunisienne Bawsala ${ }^{13}$ ou sur des questions plus thématiques comme avec différents groupes militants LGBT+. Une membre du journal décrit l'ancrage du journal dans cet environnement social.

«Les associations sont à la fois des sources d'information et des partenaires sur certains sujets, on navigue dans un tissu associatif, l'environnement de la société civile, cela nous permet d'avoir certaines informations et de valoriser leur travail.»

À cette ambition de représenter les franges populaires s'ajoute une volonté de donner au journaliste un rôle politique de lutte contre toute dérive ou concentration du pouvoir. Dans un espace journalistique national qui n'est pas à l'abri d'une restauration des logiques autoritaires (Klaus, 2017), le journalisme d'investigation est présenté comme un "contre-pouvoir» qui lutte contre "l'impunité et l'opacité des pouvoirs en place ${ }^{14}$ ». II fait émerger la figure du journaliste-justicier (Benaziz, 2015) qui révèle ce qui est caché et combat les injustices. En ce sens, ce genre journalistique se place dans une posture de dénonciation et contribue à politiser certaines thématiques (environnement, santé, transports) souvent traitées selon une vision déconflictualisée (Nollet, Schotté, 2014) par les médias traditionnels. Ainsi, les enquêtes sur la pollution à Gabès (Lac, 2019), les conditions d'hygiène dans les hôpitaux tunisiens (Zbiss, 2019) ou les accidents de train (Rédaction Inkyfada, 2010) pointent du doigt la responsabilité de

\footnotetext{
${ }^{13}$ Bawsala, ou «boussole», est une association tunisienne de veille du travail législatif et exécutif, fondée en 2011.

${ }^{14}$ Site Inkyfada, «À propos», https://inkyfada.com/fr/a-propos/
} 
groupes privés ou de l'État (Inkyfada, 2019). Les journalistes s'emparent aussi de ce qui est communément appelé les "affaires politiques», qui sont généralement le résultat de collaborations au sein de réseaux internationaux d'investigation. Ainsi, Inkyfada est le seul média tunisien à participer aux Swiss Leaks (Sbouai, Khadraoui, 2015) pour révéler des cas de fraude fiscale en Tunisie, puis aux Panama Papers (Sbaoui, Khadraoui, 2016) en dénonçant la création de sociétés offshore par des hommes politiques tunisiens ${ }^{15}$. Les journalistes "d'investigation » construisent leur légitimité professionnelle par la revendication des principes dits «traditionnels» du métier : I'objectivité, l'impartialité, la distanciation. Cela s'illustre notamment dans le style d'écriture: les reporters n'apparaissent jamais dans le récit, ils tentent de donner la parole sans se mettre en scène ou donner leur opinion. La «modernité » d'Inkyfada serait caractérisée autant par les pratiques journalistiques que par les idéaux qui l'animent. Ainsi, les journalistes se revendiquent d'une approche «intersectionnelle ${ }^{16}$ », à la fois « décoloniale» et «féministe», dont le but est d'analyser les "causes structurelles» des problèmes qui touchent la société tunisienne. Cette dimension militante se traduit également dans la manière d'écrire puisque la rédaction a fait le choix de l'écriture inclusive depuis plusieurs années.

La question de la langue est justement capitale puisqu'elle dit beaucoup sur le public visé ou sur le message transmis par le journal. Inkyfada publie en trois langues: le français, l'arabe et l'anglais. La grande majorité des articles sont écrits en français puis traduits dans les deux autres langues. En novembre 2020, seulement deux des neuf journalistes qui composent l'équipe de rédaction écrivent en arabe, et ils n'ont été recrutés que récemment. La rédaction arabophone du journal a toujours été très faible et limitée à la traduction des publications en français. Cela s'explique, selon les responsables du journal, non seulement par les lacunes du système tunisien de formation au journalisme, mais aussi par le manque de ressources humaines internes pour accompagner les jeunes recrues à la publication en arabe. Enfin, la version anglaise a été lancée en 2020 et reste principalement constituée d'articles traduits du français. Une des membres de la rédaction souligne cette prédominance de la langue française et ses limites vis-à-vis du public.

\footnotetext{
${ }^{15}$ La participation du journal à ces enquêtes internationales se résume à l'analyse des bases de données récoltées par le consortium afin d'y trouver des informations reliées à la Tunisie et pouvant déboucher sur la révélation «d'affaires».

${ }^{16}$ Entretien avec plusieurs journalistes, Tunis, novembre 2020.
} 
"On est clairement un média francophone, ça se voit dans nos publications, on produit et on travaille beaucoup plus nos articles en français qu'en arabe (...). Pourtant, je vois bien, les articles en arabe sont plus lus et partagés.»

Les responsables du journal sont bien conscients des limites de l'usage quasi exclusif du français pour un média qui se veut le porte-parole des classes populaires qui, elles, parlent et lisent peu le français. Pour répondre à ce décalage, ils ont notamment créé les "podcasts Inkyfada», des séries de documentaires sonores en arabe tunisien traitant de sujets politiques comme l'anniversaire du 14 janvier 2011 (date symbolique de la révolution et du départ de Ben Ali), les mobilisations sociales à El Kamour ou le mouvement Ena Zeda ("Me too» en arabe tunisien). Les podcasts sont construits sur des témoignages plutôt que sur une enquête journalistique, l'arabe tunisien est alors la langue privilégiée pour que les gens "se confient, partagent leurs émotions ou leurs souvenirs ${ }^{17}$ ». Selon la même logique d'élargissement du public, certaines enquêtes publiées en français sont lues en dialecte tunisien pour les rendre accessibles aux non francophones, aux gens qui ne lisent pas ou aux malvoyants. Malgré ces initiatives, le français reste la langue prédominante, et cela s'explique notamment par la sociologie des membres de la rédaction.

En novembre 2020, l'équipe d'Inkyfada était composée d'une trentaine de personnes employées à plein temps: deux à la direction, neuf dans l'équipe de rédaction, quatre à la gestion des projets de l'association $\mathrm{Al}-\mathrm{Khatt}^{18} \mathrm{et}$ une quinzaine de développeurs et graphistes au «Lab ${ }^{19}$ ». Nous nous intéresserons ici aux profils des membres de la rédaction. La grande majorité sont des femmes $(n=7)$, âgées de 20 à 35 ans, de nationalité tunisienne $(n=2)$, française $(n=1)$ ou des deux nationalités: française et tunisienne $(n=4)$. Elles maîtrisent toutes parfaitement le français, qui est leur langue maternelle ou privilégiée pour l'écriture, et le dialecte tunisien, mais très peu maîtrisent l'arabe classique. En novembre $2020^{20}$, plus de la moitié de l'équipe de rédaction a grandi en France. De parents tunisiens, ces jeunes journalistes rendent visite chaque année à leur famille en Tunisie, parlent parfois le

\footnotetext{
${ }^{17}$ Entretien avec la responsable des podcasts Inkyfada, Tunis, novembre 2020.

${ }^{18}$ Al Khatt (La ligne) est une association tunisienne créée en 2013 qui héberge le journal Inkyfada. Le statut associatif a été privilégié car il permet de recevoir des financements étrangers et de mener des projets de formation au journalisme.

19 InkyLab est le laboratoire informatique rattaché au journal. Les développeurs conçoivent des produits utilisés par la rédaction ou vendus à des clients extérieurs.

${ }^{20}$ Ces chiffres sont à analyser avec précaution puisque la composition de l'équipe du journal est en constante évolution.
} 
dialecte à la maison ou prennent des cours d'arabe. Après leurs études supérieures en France (écoles de journalisme, études en langue ou en sciences humaines), elles décident de réaliser une expérience professionnelle en Tunisie. Certaines la présentent comme un moyen de "se connecter au pays des parents», d'autres comme une manière de "voir la révolution de près». Plusieurs d'entre elles prennent des cours d'arabe classique en arrivant à Tunis avant de réaliser un stage ou une pige à Inkyfada avant d'être recrutées. Le départ pour l'autre côté de la Méditerranée est parfois mal compris par les parents tunisiens restés en France, comme le souligne une des journalistes.

«Ma mère n'a pas compris le fait que je veuille travailler en Tunisie, elle m'a dit: «Mais pourquoi tu veux aller là-bas? Regarde tout ce que la France t'offre et toi tu veux partir?» (...) Je crois qu'ils ne comprennent pas parce que c'est le schéma inverse qui se produit, ils ont dû quitter le pays à 20 ans pour rejoindre la France, et moi je fais l'inverse.»

La mobilité professionnelle en Tunisie est aussi source de questionnements et de doutes pour ces jeunes femmes journalistes formées en France. La plupart d'entre elles, n'ayant pas vécu dans le pays, s'interrogent sur leur légitimité à exercer en Tunisie. Lors de leurs reportages, certains interlocuteurs leur renvoient parfois le fait qu'elles parlent mal le tunisien ou qu'elles ne comprennent pas les enjeux locaux. Ce décalage entre une partie de l'équipe de la rédaction et ses sources, ou le public de manière générale, est un indice de la position d'Inkyfada dans le champ journalistique tunisien.

S'il apparaît comme le «bon élève» des acteurs internationaux, le média Inkyfada reste marginalisé au sein du champ journalistique tunisien. Ainsi, il ne fait pas partie de la liste des sites d'information "crédibles et intègres » publiée par la Fédération tunisienne des directeurs de journaux (FTDJ) publiée en avril 2020 (Webmanagercenter, 2020). Les sites absents de cette liste sont accusés de "propager des articles de propagande, de diffamation et de dénigrement à l'encontre d'une ou de plusieurs parties politiques » et de «ne pas respecter les normes de la profession». Le journal a également été décrié par des acteurs politiques et journalistiques, notamment lors de sa participation à l'enquête internationale des Panama Papers. Après la «révélation» de l'implication d'hommes politiques et d'hommes d'affaires tunisiens dans les affaires du cabinet Mossack Fonseca, le média a été piraté, mais il a aussi fait l'objet de critiques venant de la profession. Des confrères lui reprochaient d'agir selon un agenda politique guidé par des financements étrangers, de privilégier la logique du scoop à la rigueur professionnelle ou de ne pas partager les informations des Panama Papers avec d'autres médias tunisiens (Bahloul, 2016). La médiatisation de l'affaire révèle une double 
logique, celle de la consécration d'Inkyfada comme un média d'investigation légitimé dans son activité par les attaques politiques dont il fait l'objet, et d'une structure critiquée dans l'espace journalistique national. Enfin, la question de l'audience ajoute également à la marginalisation du média numérique qui touche principalement des jeunes âgés de 25 à 45 ans, avec un haut niveau d'éducation et majoritairement habitants de Tunis, Sousse ou Sfax, les trois plus grandes villes du pays (Foroudi, 2020).

\section{L'aide aux médias comme un instrument de politique extérieure}

Après s'être intéressé aux caractéristiques d'un média particulièrement soutenu par des acteurs étrangers, il convient maintenant de «remonter» la chaîne de l'appui aux médias en étudiant les structures qui mettent en place ces dispositifs. L'objectif de cette seconde partie est d'analyser les logiques de production de "l'aide» étrangère aux médias tunisiens. Pour cela, nous formulons I'hypothèse que ces activités de soutien sont des instruments de politique extérieure des États financeurs. Il s'agit en effet pour eux de promouvoir un certain modèle journalistique, d'identifier des partenaires locaux capables de le diffuser, de mettre à l'agenda certaines thématiques, de faire du plaidoyer auprès des autorités politiques pour réformer le cadre législatif. Cependant, cet espace de «l'aide » étrangère aux médias tunisiens est loin de représenter un tout homogène, étant plutôt mû par des agendas variés et des rapports de force mouvants. Dans un premier temps, il s'agit de restituer l'écosystème des différents acteurs intervenants dans ce secteur en Tunisie en étant attentif aux nuances entres approches nationales. Dans un second temps, nous nous concentrerons sur le cas de l'agence française de développement médias (CFI) pour comprendre ses activités.

\section{L'espace du «développement médias » en Tunisie}

L'instrumentalisation du soutien à la presse comme un outil de politique extérieure n'est pas nouvelle. L'empire espagnol appuyait financièrement le développement de journaux pro-empire dans ses colonies d'Amérique latine au XVIIe siècle, et les États-Unis encourageaient l'émergence de nouveaux médias pendant la Guerre froide en prônant la «liberté d'expression» face au "communisme» (Lugo Ocando, 2018). Aujourd'hui, ces interventions étrangères dans un champ journalistique national sont légitimées par des standards internationaux (les objectifs de développement durable de l'ONU, 
les priorités de l'Union européenne) ou au nom de valeurs prétendument universelles (liberté d'expression, pluralisme, droit à l'information et à la participation au débat public). Ces dispositifs reposent généralement sur l'idée que l'appui aux médias est un vecteur de "développement » ou de "démocratisation » d'une société (Scott, 2014). Depuis plusieurs décennies, mais surtout depuis 2011, la Tunisie a été particulièrement concernée par des politiques étrangères de "coopération", notamment dans le secteur des médias. Considéré comme «l'exception» d'un supposé «autoritarisme arabe» (Camau, 2018) après le moment révolutionnaire, le pays est devenu le principal bénéficiaire de l'aide européenne avec une aide financière avoisinant les 3,5 milliards d'euros entre 2011 et $2016^{21}$. Le secteur médiatique n'a pas échappé à cette euphorie étrangère pour le «modèle tunisien», même s'il est marginal par rapport à la sécurité, la gouvernance ou la justice. À titre d'exemple, 51 programmes ont été mis en place par des acteurs étrangers en Tunisie uniquement sur l'année $2011^{22}$. Pour comprendre les effets de ces interventions sur l'univers journalistique national, il convient d'étudier cet écosystème en détail.

L'espace du "développement médias » en Tunisie est composé de différents types d'acteurs (bailleurs de fonds, opérateurs de mise en œuvre, partenaires locaux) de nationalités différentes. Les principaux bailleurs de fonds sont les États-Unis, I'Union européenne et certains de ses États comme la France, l'Allemagne ou la Belgique ainsi que les États scandinaves ${ }^{23}$. Les organisations étrangères qui réalisent ces programmes d'appui sont principalement françaises, allemandes, britanniques, danoises ou suisses ${ }^{24}$. Elles peuvent être de statuts différents, allant de I'ONG à la fondation politique en passant par l'agence publique de développement ou le département d'un média public. Ces structures peuvent être spécialisées

\footnotetext{
${ }^{21}$ https://www.euromesco.net/wp-content/uploads/2018/04/EuroMeSCo-Joint-Policy-Study_ EU-Tunisia-Partnership.pdf

22 Tableau récapitulatif des appuis au secteur médiatique tunisien apportés par les bailleurs de fonds internationaux, publié par le Service européen pour l'action extérieure: https://docplayer. fr/8587553-Tableau-recapitulatif-des-appuis-au-secteur-mediatique-tunisien-menes-par-lesbailleurs-de-fonds-internationaux.html.

${ }^{23}$ D'autres États étrangers ont investi l'espace journalistique tunisien dans une moindre mesure ou de manière beaucoup moins transparente. La Chine et le Japon ont mis en place quelques projets auprès de médias publics, la Turquie et le Qatar soutiendraient plusieurs médias considérés comme «islamistes» selon les rapports de la HAICA.

${ }^{24}$ La grande majorité de ces organisations sont européennes et américaines. L'institut de formation de la chaîne $A$ l Jazeera mène quelques projets depuis Doha, mais il ne possède pas de locaux dédiés à la formation à Tunis.
} 
dans le secteur des médias ou généralistes, elles sont indépendantes ou affiliées à un parti ou un ministère. Pour mettre en place les activités financées par leurs bailleurs, ces organisations s'allient avec des partenaires locaux, allant des ministères aux associations de la société civile en passant par les organisations professionnelles ou les instances indépendantes.

Le principal domaine dans lequel elles interviennent est celui de la formation au journalisme, afin de promouvoir des «bonnes pratiques» et diffuser un modèle de journalisme «professionnel » défini selon leurs critères. Pour se rendre compte de la mise à l'agenda de certaines pratiques et thématiques, le cas du Centre africain de perfectionnement des journalistes et des communicateurs (CAPJC) est révélateur. Proposant des formations professionnelles à des journalistes tunisiens et plus généralement africains, ce centre est devenu le principal partenaire des organisations étrangères depuis sa création en 1983 et particulièrement à partir de 2011. Bien que sous la tutelle du chef du gouvernement, le centre dispose d'un faible budget, ce qui le contraint à faire appel à des partenaires extérieurs pour financer les formations. Par conséquent, le programme de ces formations est largement défini par les priorités des bailleurs de fonds internationaux. L'analyse des transformations du curriculum et des intitulés des formations de 2000 à 2015 permet de mettre en lumière les principales caractéristiques du modèle professionnel promu par les bailleurs (tableau 1).

Tableau 1

Nombre et intitulé des formations du CAPJC de 2000 à 2015

\begin{tabular}{|c|c|c|c|c|c|}
\hline & 2000 & 2005 & 2011 & 2013 & 2015 \\
\hline $\begin{array}{l}\text { Nombre de } \\
\text { sessions de } \\
\text { formation }\end{array}$ & 24 & 24 & 28 & 39 & 41 \\
\hline $\begin{array}{l}\text { Nombre de } \\
\text { partenaires } \\
\text { étrangers }\end{array}$ & 2 & 3 & 8 & 11 & 11 \\
\hline $\begin{array}{l}\text { Principale(s) } \\
\text { thématique(s) }\end{array}$ & \begin{tabular}{|l|} 
Formation \\
aux outils \\
numériques \\
$(\mathrm{n}=18)$
\end{tabular} & $\begin{array}{l}\text { Formation } \\
\text { aux outils } \\
\text { numériques } \\
(n=13)\end{array}$ & $\begin{array}{l}\text { - Débats et } \\
\text { reportages } \\
\text { politiques } \\
(\mathrm{n}=8) \\
\text { - Couverture } \\
\text { des élections } \\
(\mathrm{n}=4) \\
\text { - Journalisme } \\
d^{\prime} \text { investigation } \\
(\mathrm{n}=3)\end{array}$ & $\begin{array}{l}\text { - Journalisme } \\
\text { en temps de } \\
\text { conflit }(n=5) \\
\text { - Médias } \\
\text { et justice } \\
\text { transitionnelle } \\
(n=4)\end{array}$ & \begin{tabular}{|l} 
- Journalisme \\
de proximité \\
et/ou associatif \\
$(n=8)$ \\
- Journalisme et \\
décentralisation \\
$(n=6)$ \\
- Journalisme \\
environnemental \\
$(n=3)$
\end{tabular} \\
\hline
\end{tabular}

Source: Archives du CAPJC, tableau réalisé par l'auteur. 
L'activité du centre a sensiblement augmenté entre 2005 et 2015 : le nombre de sessions de formation par an a quasiment doublé. Cela s'explique par la signature de nouveaux partenariats entre le centre et des organisations étrangères arrivées en Tunisie entre 2011 et 2013. L'augmentation du nombre de partenaires s'est accompagnée d'une transformation du contenu des formations avec une rupture nette marquée en 2011. Avant cette date, la grande majorité des sessions de formation était concentrée sur des questions numériques, ce qui s'explique par le fort contrôle politique du régime de Ben Ali sur la profession, n'autorisant qu'un enseignement technique. Après le moment révolutionnaire, la formation du CAPJC est principalement orientée vers des objets politiques tels que les élections ou les débats. De 2011 à 2015, l'offre de formation se diversifie nettement et laisse entrevoir les principales caractéristiques du modèle professionnel promu par les bailleurs. Le rôle du journaliste est en phase avec le contexte de «transition démocratique»: il doit être capable de modérer les débats en période de conflit et de participer au processus de justice transitionnelle, «récit international de la réconciliation» promu par les organisations étrangères (Gobe, 2019). II est censé accompagner la « réforme» de l'État tunisien par la couverture de la décentralisation, des questions environnementales et locales.

\section{Des approches nationales variées}

Cette analyse, qui donne l'impression d'un modèle homogène diffusé par les organisations internationales, ne doit pas nous faire oublier les différences entre les politiques nationales. Chaque structure de "développement médias» adopte une stratégie propre en fonction de son statut, de ses bailleurs de fonds, de ses priorités, de son histoire dans le pays d'intervention et de ses partenaires locaux. Pour mieux se rendre compte de la diversité des cas de figure, il est utile de comparer les cas de trois opérateurs actifs en Tunisie, ce qui nous permettra ensuite d'identifier les spécificités de l'approche française qui nous intéresse particulièrement.

Les organisations choisies se distinguent d'abord par leur statut: International Media Support (IMS) est une ONG danoise, la Deutsche Welle Akademie (DWA) est un département de la chaîne de TV publique allemande du même nom, enfin, la Fondation Friedrich Neumann (FFN) est une fondation politique allemande reliée au parti libéral démocrate. Ces nuances ont un impact très concret sur les dispositifs d'appui aux médias tunisiens puisque I'ONG IMS s'inscrit dans une dimension militante de soutien à la société civile, alors que la DWA a fait le choix de collaborer avec les institutions publiques tunisiennes et que la fondation FFN s'est 
principalement tournée vers la formation au journalisme en promouvant «l'innovation». Leur histoire en Tunisie détermine également leur stratégie actuelle, puisque certaines étaient présentes à l'époque de Ben Ali et d'autres ne sont arrivées qu'à partir de 2011. Par exemple, IMS jouit d'une bonne réputation auprès de la "société civile» après avoir soutenu les défenseurs des droits sous Ben Ali ${ }^{25}$. Au contraire, la fondation FFN est parfois critiquée pour ne pas avoir dénoncé la censure de l'ancien régime, alors même qu'elle mène des activités dans le pays depuis 1964. Des organisations comme la DWA ou CFI ont, quant à elles, dû trouver leur place sur le "marché » tunisien après 2011 et faire face à des concurrents déjà bien installés. Dans un secteur saturé, chaque opérateur doit se spécialiser selon ses priorités et les possibilités offertes en Tunisie. Ainsi, la DWA fait de la décentralisation, principe politique phare du système allemand, une priorité stratégique en soutenant les radios associatives locales. L'ONG danoise entend avant tout accompagner les contre-pouvoirs que sont le syndicat des journalistes (SNJT) et les médias indépendants Nawaat et Inkyfada.

Tableau 2

Comparaison entre les stratégies des quatre structures européennes de "développement médias » en Tunisie

\begin{tabular}{|l|l|l|l|l|}
\cline { 2 - 5 } \multicolumn{1}{c|}{} & \multicolumn{1}{|c|}{$\begin{array}{c}\text { Fondation } \\
\text { Friedrich } \\
\text { Naumann }\end{array}$} & $\begin{array}{l}\text { International } \\
\text { Media } \\
\text { Support }\end{array}$ & $\begin{array}{l}\text { Deutsche Welle } \\
\text { Akademie }\end{array}$ & $\begin{array}{l}\text { Canal France } \\
\text { International }\end{array}$ \\
\hline Statut & $\begin{array}{l}\text { Fondation } \\
\text { politique }\end{array}$ & ONG & $\begin{array}{l}\text { Département de } \\
\text { média public }\end{array}$ & $\begin{array}{l}\text { Agence de } \\
\text { développement }\end{array}$ \\
\hline $\begin{array}{l}\text { Ministères } \\
\text { allemands, } \\
\text { Parti libéral } \\
\text { démocrate }\end{array}$ & $\begin{array}{l}\text { Ministères } \\
\text { norvégien } \\
\text { et danois } \\
\text { des Affaires } \\
\text { étrangères }\end{array}$ & $\begin{array}{l}\text { Ministères } \\
\text { allemands de } \\
\text { la Coopération } \\
\text { et des Affaires } \\
\text { étrangères }\end{array}$ & MEAE, UE, AFD & 2011 \\
\hline Priorité & Innovation & Plaidoyer & Décentralisation & Jeunesse \\
\hline $\begin{array}{l}\text { Partenaires } \\
\text { tunisiens }\end{array}$ & IPSI, CAPJC & $\begin{array}{l}\text { SNJT, } \\
\text { Inkyfada, } \\
\text { Nawaat }\end{array}$ & $\begin{array}{l}\text { Inkyfada, radios } \\
\text { associatives }\end{array}$ & $\begin{array}{l}\text { Inkyfada, CAPJC, } \\
\text { TAP }\end{array}$ \\
\hline
\end{tabular}

${ }^{25}$ Entretien avec la responsable des projets au Maghreb à IMS, juin 2019. 
Enfin, si la stratégie de CFI en Tunisie était d'abord orientée vers les médias publics (chaîne TV Al Wataniya, Agence Tunis Afrique Presse), elle se tourne ensuite vers la "jeunesse» en soutenant de nouveaux médias en ligne comme Inkyfada, Barr-al-aman ou le Tunisie Bondy Blog. Pour comprendre ce tournant dans la politique française, il faut désormais s'intéresser aux logiques de production de «l'aide» de CFI.

\section{La politique extérieure française de soutien aux médias}

Depuis le début des années 2010, le secteur français de «l'aide» aux médias est dominé par cette structure. Grâce à une subvention annuelle du ministère des Affaires étrangères, I'agence du Quai d'Orsay est dans une situation quasi monopolistique. Dans les années 1990 et 2000, des ONG françaises (Institut Panos, GRET) et des écoles de journalisme (ESJ Lille, (FPJ) ont pourtant mené des dispositifs de coopération avec des «médias du Sud", mais elles ne sont plus ou peu actives en 2021. Pour comprendre la spécificité de la politique française, il faut donc revenir sur l'histoire de cette structure, son positionnement entre différents univers professionnels et la sociologie de ses membres.

D'abord créé en 1989 comme une banque de programmes chargée de distribuer des images de la TV publique française à des chaînes étrangères partenaires, CFI s'est transformé au cours des années 2000 en une chaîne télé francophone particulièrement présente en Afrique. C'est à partir de 2005 que CFI devient une agence sous la tutelle du ministère français de I'Europe et des Affaires étrangères (MEAE) bénéficiant d'une subvention annuelle de l'aide publique au développement (APD) qui représente $80 \%$ de son budget ${ }^{26}$. L'organisation se situe donc à la croisée des espaces journalistique, diplomatique et de l'aide au développement. En tant que filiale du groupe France Médias Monde (FMM), CFI est directement lié à l'appareil de l'audiovisuel extérieur aux côtés de RFI, France 24 et Radio $M C D$. Son conseil d'administration est composé de quelques cadres de FMM, et plusieurs de ses formateurs sont journalistes dans ces médias. Canal France International est aussi sous l'influence de l'Agence française de développement (AFD), I'un de ses principaux bailleurs aujourd'hui derrière le MEAE. L'AFD s'intéresse de plus en plus au secteur des médias et finance les

\footnotetext{
${ }^{26}$ La subvention annuelle du ministère s'est stabilisée autour de 7 millions d'euros depuis plusieurs années.
} 
plus importants projets de $\mathrm{CFI}^{27}$. Enfin, l'influence de l'Union européenne est croissante pour cette organisation qui cherche à diversifier ses financements et se tourne donc vers les grands projets européens notamment consacrés au «monde arabe». À titre d'exemple en Tunisie, CFI a mené par deux fois des consortiums internationaux pour obtenir des projets européens d'appui aux médias d'une valeur de 10 millions d'euros. Si l'organisation est donc inscrite dans différentes relations d'interdépendance, l'allégeance la plus importante reste celle au MEAE.

CFI a été pensé dès sa création comme un outil diplomatique de la France pour exercer son influence à l'étranger. Initié par le ministère de la Coopération, l'opérateur passe ensuite sous la tutelle du Quai d'Orsay en 2009. En 2021, la direction générale de la mondialisation (DGM) du Quai d'Orsay donne les priorités stratégiques à son agence. L'équipe de cinq personnes du pôle "Médias et cinéma» installée dans les locaux du $15^{\mathrm{e}}$ arrondissement de Paris du MEAE a pour tâche de coordonner la politique française de «coopération médias» à l'étranger. Elle donne les grandes orientations thématiques et géographiques des activités de CFI par un contrat d'objectifs renouvelé tous les trois ans. C'est elle qui propose le nom du directeur général de $\mathrm{CFI}$, qui doit d'ailleurs être un diplomate du Quai, ce qui permet de préserver cette allégeance à la tutelle. Elle lui envoie une lettre d'objectifs chaque année afin de traduire la parole du président de la République en objectifs stratégiques pour CFI. Ainsi, les discours de Ouagadougou $^{28}$ et de la Sorbonne ${ }^{29}$ du président Emmanuel Macron ont défini la stratégie de CFI ces dernières années, en mettant l'accent sur la zone géographique du Sahel et sur la thématique de la francophonie. $\mathrm{Si}$ le MEAE définit donc les grandes lignes stratégiques de l'action de CFI, la relation qui lie les deux structures reste très souple. Dans les faits, la stratégie de l'agence est définie quotidiennement par ses membres en parallèle des orientations du MEAE qui restent très vagues.

Les trajectoires sociales et professionnelles de ses membres sont de bons indicateurs pour comprendre comment l'action de CFI se construit au

\footnotetext{
${ }^{27}$ On pense ici aux projets "Qarib» au Moyen-Orient d'un budget de 10 millions d'euros et "Médias Sahel » de 8,3 millions d'euros.

${ }^{28}$ Discours d'Emmanuel Macron le 28 novembre 2017 à Ouagadougou. https://www.elysee.fr/ emmanuel-macron/2017/11/28/discours-demmanuel-macron-a-luniversite-de-ouagadougou ${ }^{29}$ Discours d'Emmanuel Macron le 27 août 2018 à la Sorbonne. https://www.elysee.fr/ emmanuel-macron/2018/08/27/discours-du-president-de-la-republique-a-la-conference-desambassadeurs
} 
quotidien. L'espace de ces acteurs reflète l'ancrage de CFI dans différents univers et les logiques professionnelles variées - voire contradictoires - qui traversent son activité. Nous avons identifié trois trajectoires récurrentes des employés de l'agence en leur donnant une appellation. Les «baroudeurs» ont mené une carrière dans I'humanitaire et ont multiplié les expériences à l'étranger (notamment en Afrique). Ils se distinguent par leur bonne connaissance de l'ingénierie des projets de développement et leurs vastes réseaux dans les pays d'intervention. Les "experts techniques» sont diplômés de masters "coopération» et ont eu des expériences dans des ONG ou fondations internationales, ce qui leur a permis d'acquérir des compétences techniques très recherchées (réponse à appel à projets, constitution d'un cadre logique, montage d'un budget, rédaction des «termes de référence»). Enfin, les «diplomates des médias» sont d'anciens ambassadeurs ou attachés audiovisuels à l'étranger, réputés pour leur réseau d'officiels et leur aisance orale, connaissant les rouages de l'appareil diplomatique français et s'étant spécialisés dans le secteur médiatique.

Loin d'être un bloc homogène, "l'aide » internationale aux médias apparaît donc comme un espace traversé par des logiques de concurrence, des rapports de force, des visions différentes de ce que devrait être le journalisme. Le cas de CFI révèle la diversité des influences: une agence du Quai d'Orsay, dépendante de I'AFD et de I'UE, rattachée à un groupe de médias. La politique française de soutien aux médias évolue donc au fil des trajectoires professionnelles de ses agents, tout en ayant un effet sur ces dernières. Cette nécessaire prise en compte de la coproduction de l'aide nous permet d'en analyser les effets sur la profession journalistique du pays d'intervention.

\section{Effets de l'aide et appropriation par les journalistes}

Analyser les effets des dispositifs internationaux sur la profession journalistique suppose de mettre à distance deux hypothèses qui fondent un certain nombre de travaux sur les politiques de développement (Nay, 2010): se défaire de l'idée selon laquelle l'appui étranger exercerait une influence directe sur les organisations locales au point où ces dernières adopteraient totalement les codes extérieurs et perdraient ainsi toute autonomie; à l'inverse, réfuter l'hypothèse affirmant que les acteurs nationaux seraient libres de toutes contraintes, capables de s'opposer à I'influence des bailleurs quand elles le jugent légitime. Notre démarche 
s'inscrit plutôt dans la continuité des travaux qui portent une attention particulière aux effets du développement sur les pratiques, les discours ou les trajectoires biographiques (Parizet, 2015) en se concentrant sur des acteurs intermédiaires qui permettent de nuancer l'opposition entre «Nord» et «Sud». Tout d'abord, il s'agit d'analyser la manière dont l'aide étrangère contribue à transformer les pratiques journalistiques, la conception du métier et la sociologie des membres du journal Inkyfada. Ensuite, nous mettrons en lumière les stratégies de ré-appropriation adoptées par les journalistes qui soulignent leur marge de manœuvre et font d'eux des coproducteurs de l'aide plutôt que des «bénéficiaires».

\section{Écarter les journalistes des activités éditoriales}

Force est de constater que les instruments de «développement médias » contribuent paradoxalement à éloigner les journalistes du travail éditorial censé représenter leur principale activité. Ce constat repose sur un travail empirique réalisé auprès du journal tunisien Inkyfada en observant les activités quotidiennes de ses membres (reportages de terrain, ateliers de formation, suivis de projet) et en analysant leur trajectoire biographique. L'analyse de la répartition des activités au sein du média montre la part importante des tâches non éditoriales qui relèvent plus de la gestion, de la communication ou de l'évaluation que du journalisme. Elle montre également que la division du travail se base sur une nette rupture dans la sociologie des membres.

L'insertion de Inkyfada dans le champ de l'aide internationale fait que le journal vit au rythme des "projets». Les journalistes adoptent les codes linguistiques propres au secteur du développement et réalisent les tâches caractéristiques de l'ingénierie des dispositifs internationaux. Les membres du média doivent d'abord assurer une activité de veille afin d'identifier les potentielles sources de financement, écrire des proposals (documents de présentation) en réponse aux appels à projets des bailleurs, monter des budgets et des programmes d'activité pluriannuels, réaliser des audits ou des évaluations pour identifier les «besoins» auxquels répondre. Une fois que le partenariat avec un bailleur étranger est signé, la charge administrative est toujours aussi conséquente pour les journalistes qui doivent faire des rapports réguliers (reporting) de leurs activités, évaluer leur impact et justifier des "résultats mesurables» aux bailleurs, faire preuve d'«innovation » pour proposer de nouvelles activités. Considéré comme un partenaire tunisien de référence par de nombreux bailleurs 
étrangers, Inkyfada est devenu une sorte d'opérateur local auquel on délègue des tâches qui ne sont en général pas attribuées à un média spécifique. Ainsi, les membres sont amenés à organiser et mener des formations, à sélectionner des journalistes «bénéficiaires » pour des projets de développement, à structurer des réseaux de professionnels. Ils sont également encouragés à présenter le modèle du journal à l'étranger lors de conférences internationales, à conseiller des opérateurs sur leur stratégie, voire à rédiger avec eux de nouveaux projets.

Afin de gérer cette charge de travail et de ne pas détourner les journalistes de leur mission, la direction a opté pour une division des tâches entre l'équipe éditoriale d'Inkyfada et une équipe dédiée à la gestion de projet et rattachée à l'association Al-Khatt. L'observation des pratiques quotidiennes des journalistes révèle pourtant qu'ils sont aussi amenés à réaliser des activités non éditoriales que les quatre membres de l'association ne peuvent pas gérer. À ce titre est instructif le témoignage d'une ancienne membre de la rédaction chargée de réaliser une étude pour un projet avec une ONG sur la propriété des médias en Tunisie.

"Au moment où la personne de l'équipe chargée de ce projet est partie, j'ai proposé mon aide parce que j'aime apprendre de nouvelles choses et je voulais me sentir utile. Au début, c'était juste ponctuellement, puis j'ai pris une place de plus en plus importante dans le projet (...). Pendant plusieurs mois, j'ai passé la plupart de mon temps sur ce dossier: j'étais chargée du tri des informations, des entretiens, de la mise en page sur le site, du montage du budget (...). Mais, en même temps, je devais continuer à produire des articles.»

Bien conscients du risque que représente cette charge de travail sur l'équipe de rédaction, les membres de la direction tentent de délimiter la frontière entre le journal et l'association, la production d'articles et la gestion de projets. Ils ne cherchent plus à recruter des profils «multicasquettes ${ }^{30}$ » capables d'assurer toutes ces tâches, mais plutôt de distinguer les compétences du «bon journaliste» et celles du «bon gestionnaire de projet». C'est ce qui explique la distinction claire entre deux groupes d'individus aux propriétés sociales et linguistiques différentes.

Au sein de l'équipe de rédaction d'Inkyfada sont valorisés les profils qui «savent écrire ${ }^{31}$ » et particulièrement en langue française. On attend des nouvelles recrues qu'elles maîtrisent les codes de l'écriture d'un article

\footnotetext{
${ }^{30}$ Entretien avec un responsable du journal, Tunis, mai 2019.

31 lbid.
} 
de presse, qu'elles possèdent les «réflexes» du journaliste. Cela se traduit concrètement par la sélection de jeunes diplômé.e.s d'universités et d'écoles de journalisme en France dont la formation est particulièrement valorisée par la direction du journal, au contraire de l'enseignement dispensé à I'Institut de presse et des sciences de l'information (IPSI) de Tunis ${ }^{32}$. Les recrues de la rédaction se distinguent donc par leur capital scolaire élevé, elles sont majoritairement titulaires d'un master 2 en école de journalisme, de sciences humaines ou de science politique. Si elles ne sont qu'au début de leur expérience professionnelle, elles ont réalisé des stages dans des structures médiatiques de renom en France (Le Monde diplomatique, l'émission de France Télévisions "Cash Investigation », La Croix, Society) ou à l'étranger (L'Orient-Le Jour au Liban). Les études supérieures en France ainsi que les expériences dans des médias de référence leur permettent d'afficher une maîtrise des codes du métier et de la langue particulièrement recherchée par le journal. Les trajectoires biographiques des journalistes se distinguent par une forte mobilité internationale plutôt que par des réseaux tunisiens. Parmi les plus récentes recrues, l'une est fille d'expatriés et a grandi en Palestine, les deux autres ont fait leur année à l'étranger au Canada et aux États-Unis. La plupart des membres de la rédaction n'avaient pas ou peu vécu en Tunisie avant leur expérience à Inkyfada et conservaient un lien ponctuel avec le pays. Seulement trois membres de la rédaction ont grandi et réalisé leurs études en Tunisie.

La question de l'ancrage local est donc cruciale pour ce journal qui se voit souvent attribuer l'image d'une rédaction trop francophone ou détachée des réalités tunisiennes. La direction fait donc le choix de privilégier les profils de Franco-tunisiennes, qui maîtrisent les deux langues et disposent de certains réseaux familiaux dans le pays sur lesquels elles peuvent s'appuyer. Pour autant, les journalistes se posent fréquemment la question de leur légitimité à produire de l'information sur un pays qu'elles connaissent assez peu. L'une d'elles se demandait en entretien: «Qui suis-je en tant que Française qui vient d'arriver pour décrypter le cinéma tunisien ou parler des violences faites aux femmes dans le pays? "Au-delà du décalage géographique dû aux trajectoires sociales de journalistes formés en France se pose aussi la question du décalage social d'une rédaction qui forme une certaine élite. En effet, l'équipe est composée de Franco-tunisiens qui, bien qu'issus de milieux sociaux variés en France, disposent d'un capital économique important en Tunisie et de journalistes tunisiens souvent issus

32 Un seul journaliste sur les neuf qui composent la rédaction a été formé à l'IPSI. 
de la classe moyenne, voire supérieure, ayant grandi dans la capitale. Au quotidien, ce groupe fréquente surtout les fractions dominantes de l'espace tunisois et des expatriés étrangers installés à Tunis. L'inscription dans cet environnement social privilégié peut avoir un effet sur la production du média, comme le remarque une journaliste.

"Je crois que la spécificité d'Inkyfada, c'est d'être inséré dans un environnement fait d'associations, d'ONG internationales, de centres de recherche, d'ambassades; on fréquente une certaine élite internationale que ne fréquente pas forcément un journaliste d'un autre média (...); ma manière d'aborder les sujets est sûrement en partie déterminée par cet entourage, par ces réseaux interpersonnels de chercheurs, de militants, ce sont des sources de sujets, d'informations, de réflexions.»

La sociologie des membres de l'association chargée des activités non éditoriales est sensiblement différente. Le groupe est composé uniquement de Tunisiens ayant grandi dans le pays et maîtrisant parfaitement l'arabe et le français. Ils sont soit diplômés d'une formation en journalisme de I'IPSI ou journalistes de profession, après des expériences dans des médias locaux (notamment à la radio). Leur parcours universitaire n'est pas autant valorisé que celui des premiers et semble en décalage avec leur activité actuelle; l'une est diplômée d'une école de finances tandis qu'un autre a fait des études d'infirmier. Ce sont plutôt leurs réseaux locaux, notamment dans le milieu journalistique, et leurs connaissances des problématiques politiques nationales qui fondent leur légitimité. Ainsi, l'un des membres de l'association est le seul du journal à être encarté au Syndicat national des journalistes tunisiens (SNJT), et il se montre particulièrement actif dans les mobilisations de la profession contrairement à l'équipe de rédaction qui collabore très peu avec des médias tunisiens. Chargé de coordonner un projet de formation des jeunes des régions tunisiennes, son activité proprement journalistique est limitée. Parce qu'il connaît les rouages des institutions publiques tunisiennes (par son expérience avec le ministère de l'Éducation, partenaire du projet évoqué au-dessus) et qu'il maîtrise parfaitement le dialecte tunisien, il est responsable des procédures de demande d'accès à l'information auprès des ministères et de I'Instance nationale d'accès à l'information (INAI ${ }^{33}$ ). II lui arrive également d'accompagner les journalistes de la rédaction dans leurs enquêtes. En

\footnotetext{
${ }^{33}$ L'INAI est I'Instance nationale d'accès à l'information. Instance indépendante créée en 2016, elle est censée garantir l'exercice du droit constitutionnel de l'accès à l'information pour tout citoyen.
} 
reportage dans sa ville d'origine, il met ses contacts locaux au service de la journaliste chargée de rédiger un article. Ainsi, s'il joue le rôle de fixeur, de photographe ou d'interlocuteur avec les institutions, il est très rarement l'auteur principal d'une enquête. Une journaliste de la rédaction reconnaît pourtant que, «c'est un profil rare, il est bien inséré dans les régions, arrive à se mettre à la place des gens, il comprend leurs problèmes, il faut valoriser ses compétences». Même s'ils sont journalistes de formation ou de métier, ils sont chargés de la coordination des projets de l'association et de la relation avec les bailleurs de fonds. Ce rôle les amène parfois à représenter le journal ou faire des formations à l'étranger, les bailleurs permettant alors une mobilité internationale difficile à obtenir pour des Tunisiens.

Les tâches non éditoriales liées à l'aide internationale font donc l'objet d'une répartition en interne, ce qui entraîne de facto une rupture entre deux groupes socialement distincts. D'un côté, la production écrite est réservée à des profils au capital scolaire élevé, généralement formés en France, maîtrisant les codes de la profession mais peu ancrés localement. De l'autre, la gestion des projets est réalisée par des jeunes formés en Tunisie, disposant d'un «savoir local» (Allal, 2010) leur permettant d'articuler les préoccupations des bailleurs étrangers aux besoins nationaux.

\section{Autonomie et stratégies de réappropriation}

Les relations qui lient Inkyfada à l'univers du développement pourraient laisser penser que le journal en ligne contribue à reproduire un «modèle» journalistique défini à l'étranger et donc à uniformiser les pratiques professionnelles selon ces normes. Pourtant, les entretiens avec les journalistes ainsi que l'observation de leur travail quotidien montrent qu'ils réorientent l'appui étranger selon leurs intérêts. Ils négocient leur autonomie au quotidien au contact des bailleurs de fonds, à la fois sur leur modèle économique, leur ligne éditoriale et les projets de développement qu'ils mènent.

En effet, l'autonomie d'un média comme Inkyfada réside d'abord dans sa capacité à «choisir» ses partenaires étrangers et à influer sur les modalités de la collaboration pour éviter un détournement de son identité. Le modèle économique du journal repose sur un nombre restreint de bailleurs de fonds considérés comme des "partenaires de confiance » par la direction. Le dispositif français a d'abord occupé une place primordiale dans le lancement du média puisque l'ambassade de France en Tunisie a été le premier acteur étranger à soutenir le journal par une subvention de 20000 euros en 2014. 
Cette somme d'argent, octroyée par l'attachée audiovisuelle de l'époque, a permis aux journalistes de louer les premiers locaux, d'acheter du matériel et de lancer le site internet. La France a ensuite réitéré son soutien à Inkyfada par l'action de son opérateur CFI. En charge du programme EBTICAR ${ }^{34}$, financé par l'Union européenne et orienté vers les «médias numériques innovants dans le monde arabe», CFI a attribué une subvention de 80000 euros à Inkyfada entre 2014 et 2017.

Afin de protéger la structure d'une orientation imposée par un partenaire étranger, les dirigeants du journal ont aussi décidé de privilégier les financements «structurels» aux financements dits «par projet». Les premiers fournissent des fonds nécessaires au fonctionnement de la structure indépendamment de tout projet, alors que les seconds permettent le financement d'un " projet» autour de certaines activités précises qui doivent être réalisées. En 2018, 40 \% des revenus proviennent des financements structurels de deux bailleurs: I'ONG danoise International Media Support et la fondation américaine Open Society Foundation ${ }^{35}$. Un responsable du journal en ligne explique les avantages de ce mode de revenu.

"On a imposé aux organisations internationales l'idée que nous n'étions pas un prestataire lambda, mais un vrai partenaire, et donc qu'il fallait financer la structure et les services qu'il propose plutôt que certains projets (...); c'est un modèle plus efficace, qui permet de gagner du temps en faisait moins de proposals (propositions de projets), de bilans financiers ou d'audits.»

Mais l'autonomie d'Inkyfada à l'égard de l'aide internationale se réalise surtout par la place de plus en plus importante que prennent les sources alternatives de revenus. Le reste des recettes est généré par deux activités principales: les activités de formation et de conseil, ainsi que l'offre de services informatiques. Le média a développé une expertise interne et loue une partie de ses locaux pour des formations, ce qui lui permet de générer $37 \%$ de ses revenus. Enfin, plus de $20 \%$ des recettes du journal sont issues de son laboratoire de recherche et de développement appelé «InkyLab». Les développeurs et graphistes conçoivent et vendent des produits (vidéos, infographies, cartes interactives, logiciels), proposent des services (création

\footnotetext{
${ }^{34}$ Le nom du programme «EBTICAR» renvoie à l'acronyme "E-Booster for Technical and Innovative Contents in the Arab Region » et signifie «innovation » en arabe. C'est un programme de 2,3 millions d'euros financé par I'Union européenne et mis en œuvre par CFI entre 2014 et 2016.

${ }^{35}$ Document de présentation de l'association destiné aux bailleurs de fonds.
} 
de sites web) à des ONG ou d'autres médias ${ }^{36}$. Grâce à ces activités, la part du revenu issue des financements structurels de bailleurs étrangers est passée de $60 \%$ à $40 \%$ entre 2016 et $2018^{37}$.

Le degré d'autonomie du journal se mesure également à sa ligne éditoriale. Les témoignages des journalistes vont tous dans le sens d'une totale liberté dans le choix des sujets traités, et aucune forme de censure ou d'imposition par les bailleurs n'a été reportée. En effet, il n'est pas dans l'intérêt de ces structures, qui soutiennent «l'indépendance » et la «liberté » de ces médias, de leur dicter leur ligne éditoriale. Preuve de la liberté de ton du média, plusieurs articles se montrent critiques à l'égard de I'Union européenne ou des autorités politiques françaises, pourtant bailleurs indirects. En novembre 2020, un article pointe du doigt le silence de l'ambassade française en Tunisie (Mzalouat, 2020) face aux demandes de I'Instance Vérité et Dignité (IVD) concernant les réparations aux victimes de la bataille de Bizerte de $1961^{38}$ et provoque des réactions de l'ambassade. Quelques mois plus tôt, un responsable du journal critique publiquement son partenaire CFI pour avoir provoqué l'arrestation d'un journaliste béninois ${ }^{39}$.

Enfin, l'autonomie du journal se construit sur les stratégies de réappropriation de l'aide étrangère mises en place par les membres du média. Contrairement à leurs interlocuteurs internationaux qui gèrent plusieurs programmes en même temps, les membres d'Inkyfada sont investis quotidiennement sur les quelques projets de développement. Ce décalage d'investissement entre les acteurs locaux et les acteurs internationaux (De Sardan, 1995) donne aux premiers un avantage puisqu'ils font des petits choix quotidiens qui orientent les activités d'une certaine manière, face à l'absence récurrente du bailleur. Tout en veillant à «cocher les cases» des financeurs en respectant leurs objectifs stratégiques, ils sont largement maîtres de la mise en œuvre des activités. Dans le cas d'un programme de formation de jeunes des régions tunisiennes au journalisme, les membres

\footnotetext{
${ }^{36}$ Document de présentation de l'association destiné aux bailleurs de fonds.

${ }^{37}$ Les chiffres ont été fournis par les responsables du journal et sont mis en avant dans différents documents de présentation.

${ }^{38}$ Conflit diplomatique entre la France et la Tunisie après I'indépendance du pays, qui se transforme en bataille militaire avec le bombardement français d'une partie de la ville de Bizerte, tuant de nombreux civils.

${ }^{39}$ Le journaliste a été arrêté et emprisonné après avoir participé à une conférence organisée par CFI au Bénin. Au cours de l'événement, le journaliste a critiqué le procureur général sans recevoir le soutien de $\mathrm{CFI}$, qui a condamné ses propos.
} 
de l'association décident à la fois des thématiques sur lesquelles ils veulent travailler, des exercices à mettre en place, de leurs partenaires ou encore de la forme du produit final. Mis à part le travail de reporting qu'ils doivent faire auprès du bailleur tous les trois mois, ils restent largement libres dans leurs activités quotidiennes à partir du budget délivré. Le témoignage de I'une des coordinatrices du projet est révélateur des marges de manœuvre dont disposent les membres de l'association:

«Notre partenaire étranger n'est tellement pas présent dans nos activités quotidiennes que je me dis souvent qu'il faudrait lui montrer ce qu'on fait concrètement, donc j'insiste pour que l'on ajoute des sous-titres aux vidéos produites en arabe par les jeunes, pour qu'il voie ce qu'on fait.»

Loin de se voir imposer des thématiques ou des manières de faire, les chargés de projet de l'association définissent leur propre cadre d'action et se montrent parfois critiques à l'égard des bailleurs. Dotés d'une connaissance du contexte tunisien que l'interlocuteur étranger n'a généralement pas, ils se sentent suffisamment à l'aise pour lui signifier que certains objectifs semblent en décalage avec les besoins locaux. Le témoignage de l'une d'entre elles révèle justement la marge de négociation qu'ont ces acteurs locaux à l'égard des financeurs.

«ll y a juste une fois où il (l'interlocuteur employé du bailleur) a proposé une thématique, en nous disant que l'on pourrait faire des vidéos avec les jeunes des régions sur les migrations, pour savoir ce qu'ils en pensent (...) je lui ai bien fait comprendre que si on faisait des vidéos sur cette thématique, ça n'allait pas du tout plaire à I'UE, à la Tunisie, à personne (...); on ne va pas faire des vidéos de success story (histoire de succès) de migrants qui réussissent en France, c'est horrible de faire ça (...); si on parle de migration, alors il faut dire que les gens n'ont plus rien à perdre, s'ils prennent la mer c'est parce qu'on ne les laisse pas partir légalement (...). Il a compris et n'a pas insisté.»

Ce chapitre avait donc pour objectif d'analyser la fabrique d'un soutien extérieur et ses effets sur la profession journalistique en Tunisie. À partir du cas de l'agence française de "développement médias», nous avons réfuté l'idée d'une aide étrangère qui serait homogène en soulignant les spécificités nationales et les nombreuses relations d'interdépendance dans lesquelles s'inscrivent ces organisations. Dans le cas particulier du système français de soutien aux médias, CFI apparaît comme un instrument diplomatique inséré dans l'appareil de l'audiovisuel extérieur dont le but est de promouvoir l'expertise et les «valeurs» françaises à l'étranger. Pour autant, en étudiant le cas d'un journal tunisien considéré 
comme une référence professionnelle pour les acteurs étrangers, force est de constater qu'il n'y avait pas d'imposition mécanique d'un modèle de journalisme ou d'une certaine ligne éditoriale. L'enquête montre plutôt des effets plus subtils que peuvent avoir ces dispositifs internationaux à la fois sur la sociologie des membres, la division du travail et les pratiques professionnelles. Elle révèle également le poids des acteurs intermédiaires dans ces dispositifs de développement qui s'approprient des normes extérieures et les orientent par leur travail quotidien. Ce dernier postulat reste cependant à nuancer pour le reste de l'univers journalistique tunisien, puisque le journal Inkyfada est dans une position dominante dans l'espace de l'aide aux médias et possède les ressources nécessaires pour négocier avec ses interlocuteurs étrangers. Pour résumer, les acteurs étrangers et extérieurs au champ journalistique tunisien participent activement au processus de reconfiguration de l'espace médiatique en Tunisie. En promouvant certaines pratiques et normes professionnelles, ils contribuent à redéfinir les frontières du métier. En analysant les transformations d'un média «bénéficiaire» de l'aide, cette contribution aux travaux de sociologie politique sur le développement pourrait servir de point de comparaison avec d'autres objets des politiques de développement.

\section{Bibliographie}

ALI ELHAOU Mohamed, "Le travail journalistique à I'heure de la rhétorique de la "bonne gouvernance des médias" », Communication, vol. 35, n² 2018. DOI: https://doi.org/10.4000/communication. 8654

AlLAL Amin, "Les configurations développementistes internationales au Maroc et en Tunisie : des policy transfers à portée limitée», Critique internationale, $\mathrm{n}^{\circ}$ 48, 2010. DOl: https://doi.org/10.3917/crii.048.0097

AtLANI-DuAULt Laëtitia, Au bonheur des autres: anthropologie de l'aide humanitaire, Paris, Armand Colin, 2009.

BENAZIZ Bachir, «Le journal Al-Dustour/Al-Tahrir: apogée et déclin d'un journal privé », Revue Tiers Monde, n²22, 2015. DOI: https://doi.org/10.3917/ rtm.222.0031

BENAZIZ Bachir, «Récit journalistique et action collective dans I'Égypte des années $2000 »$, Égypte/Monde arabe, n 12, 2015. DOI: https://doi. org/10.4000/ema.3410

BEN MAMI Amine, GOBE Eric, "A new offer of commitment and work for qualified youth? Democratic monitoring associations in post-Ben Ali 
Tunisia», The Journal of North African Studies, vol. 26, 2021. DOI: https:// doi.org/10.1080/13629387.2019.1665287

BenNANI-Chraï̈I Mounia, FILlIeUle Olivier, «Pour une sociologie des situations révolutionnaires: retour sur les révoltes arabes», Revue française de science politique, vol. 62, 2012. DOI: https://doi.org/10.3917/rfsp.625.767

BENSON Rodney, "Le journalisme à but non lucratif aux États-Unis: un secteur sous la double contrainte de la «viabilité » et de «l'impact», Savoir/Agir, n 46,2018 . DOI: https://doi.org/10.3917/sava.046.0089

BUNCE Mel, SCOTT Martin, WRIGHT Kate, "Foundation funding and the boundaries of journalism », Journalism Studies, $n^{\circ} 20,2019$. DOI: https:// doi.org/10.1080/1461670X.2018.1556321

CAMAU Michel, L'exception tunisienne: variations sur un mythe, Paris, KarthalaIRMC, 2018.

ChAmPAGNe Patrick, «La double dépendance: quelques remarques sur les rapports entre les champs politique, économique et journalistique», Hermès, nos 17-18, 1995. http://documents.irevues.inist.fr/bitstream/ handle/2042/15218/HERMES_1995_17-18_215.pdf

CHOUIKHA Larbi, Des séquelles de l'étatisation aux aléas de la transition. La difficile transformation des médias. Des années de l'indépendance à la veille des élections de 2014, Tunis, Editions Finzi, 2015.

FERRON Benjamin, "Giving voice to the voiceless? The ambivalent institutionalization of 'minorities' alternative media in Mexico and Israel/ Palestine», dans Mediating cultural diversity in a globalized public space, sous la dir. de I. Rigoni et E. Saïtta, Palgrave, 2012.

GOBE Éric, Justice et réconciliation dans le Maghreb post-révoltes arabes, TunisParis, IRMC-Karthala, 2020.

HIBOU Béatrice, «Les marges de manœuvre d'un bon élève économique: la Tunisie de Ben Ali», Etudes du CERI, n 60, 1999. https://www.sciencespo.fr/ ceri/fr/content/les-marges-de-manoeuvre-dun-bon-eleve-economiquela-tunisie-de-ben-ali

HMED Choukri, «Au-delà de l'exception tunisienne: les failles et les risques du processus révolutionnaire», Pouvoirs, n 156, 2016. DOI: https://doi. org/10.3917/pouv.156.0137

JUHEM Philippe, "La légitimation de la cause humanitaire: un discours sans adversaires», Mots, 2001, vol. 65, n 1. DOI: https://doi.org/10.3406/ mots.2001.2484 
KLAUS Enrique, «La restauration autoritaire au prisme des instruments de propagande: le cas de l'agence Tunis Afrique Presse (TAP) », Politique africaine, $\mathrm{n}^{\circ}$ 146, 2017. DOI: https://doi.org/10.3917/polaf.146.0049

KLAUS Enrique, «La polémique du «Livre noir des journalistes »: la justice transitionnelle tunisienne à l'épreuve de la polysémie démocratique», dans Justice et réconciliation dans le Maghreb post-révoltes arabes, sous la dir. de E. Gobe, Paris, Karthala, 2019.

KLAUS Enrique, KoCH Olivier, «Les presses privées post-2011 en Tunisie: mutation économiques et politiques ", Questions de communication, $n^{\circ} 32$, 2017. DOI: https://doi.org/10.4000/questionsdecommunication.11547

KLAUS Enrique, KoCH Olivier (dir.), Médias et recompositions politiques dans la Tunisie post-Ben Ali, Paris, IRMC-Karthala, 2020.

KocH Olivier, "Les médias dans les transitions démocratiques : état des lieux et prospective», Questions de communication, n² 28, 2015. DOI: https:// doi.org/10.4000/questionsdecommunication.10108

$\mathrm{KoCH}$ Olivier, «La (re-)professionnalisation du journalisme tunisien dans la période transitionnelle: le rôle des acteurs extranationaux", dans La Circulation des productions culturelles : cinémas, informations et séries télévisées dans les mondes arabes et musulmans, sous la dir. de D. Marchetti, Istanbul-Rabat, Éditions du Centre-Jacques Berque, 2017. DOI : https://doi.org/10.4000/books.cjb.1219

LECOMTE Romain, «Expression politique et activisme en ligne en contexte autoritaire: une analyse du cas tunisien », Réseaux, n 181, 2013. DOI: https://doi.org/10.3917/res.181.0051

LUGO-OCANDO Jairo, «A mouthpiece for truth: Foreign Aid for Media Development and the making of journalism in the Global South », Brazilian journalism research, $\mathrm{n}^{\circ}$ 14, 2018.

MARCHETTI Dominique, "Les révélations du "journalisme d'investigation" ", Actes de la recherche en sciences sociales, nos 131-132, 2000. DOI: https:// doi.org/10.3406/arss.2000.2663

MATTELART Tristan, «De la nécessité d'interroger le rôle que jouent les (nouveaux) médias dans les transitions", in Médias et recompositions politiques dans la Tunisie post-Ben Ali, sous la dir. d'E. Klaus et O. Koch, Paris, IRMC-Karthala, 2020. https://www.researchgate.net/ publication/348559218_De_la_necessite_d\%27interroger_le_role_que_ jouent_les_nouveaux_medias_dans_les_transitions 
NOLLET Jérémie, SCHOtTÉ Manuel, «Journalisme et dépolitisation», Savoir/ Agir, n² 28, 2014. DOI: https://doi.org/10.3917/sava.028.0009

NAY Olivier, "Chapitre 5 / Les politiques de développement», dans Politiques publiques 2. Changer la société, sous la dir. de O. Borraz, Presses de Sciences Po, 2010.DOI : https://doi.org/10.3917/scpo.borra.2010.01.0139

Olivier de SARDAN Jean-Pierre, Anthropologie et développement: essai en socioanthropologie du changement social, Paris, Karthala, 1995.

PARIZET Raphaëlle, Les Paradoxes du développement: sociologie politique des dispositifs de normalisation des populations indiennes au Mexique, Paris, Dalloz, 2015.

ScotT Martin, Media and Development, London, Development Matters, 2014.

\section{Sources électroniques}

Bahloul Nizar, "Inkyfada dans la tourmente des Panama Papers", Businessnews, 5 avril 2016. https://www.businessnews.com.tn/inkyfadadans-la-tourmente-des-panama-papers,519,63622,3

BEN HAMADI Monia, «À qui profite le contenu des pages Facebook tunisiennes liées à Israël? », Inkyfada, 3 juin 2019. https://inkyfada.com/fr/2019/06/03/ tunisie-facebook-israel/

FOROUDI Layli, «The Tunisian Journalists who built a business model that frees them to investigate», GIJN, 20 mai 2020. https://gijn.org/2020/05/20/ the-tunisian-journalists-who-built-a-business-model-that-frees-themto-investigate/

INKYFADA, «Signal d'alarme», 15 février 2019. https://inkyfada.com/fr/webdoc/ signal-dalarme/

LAC Hortense, "Autour du groupe chimique de Gabès, une population sacrifiée », Inkyfada, 12 novembre 2019. https://inkyfada.com/ $\mathrm{fr} / 2019 / 11 / 12 /$ pollution-gabes-lutte-gct/

MzALOUAT Haïfa, «Bizerte: au-delà du dernier soldat français évacué, une bataille oubliée», Inkyfada, 15 octobre 2020. https://inkyfada.com/ $\mathrm{fr} / 2020 / 10 / 15 /$ bataille-bizerte-france-tunisie/

SBoual Sana, KHADRAOUI Malek, "SwissLeaks : que révèlent les listings tunisiens ?», Inkyfada, 8 février 2015. https://inkyfada.com/fr/2015/02/08/ swissleaks-listings-tunisie-hsbc-fraude/

Sboual Sana, KHADRAOUI Malek, «Panama Papers, plongée dans le monde de la finance offshore», Inkyfada, 3 avril 2016. https://inkyfada.com/ 
fr/2016/04/03/panamapapers-panama-mossack-fonseca-offshore-bankicij-tunisie/

WEBMANAGERCENTER, "Liste des journaux électroniques reconnus par la Fédération tunisienne des directeurs de journaux», webmanagercenter.com, 16 avril 2020. https://www.webmanagercenter.com/2020/04/16/448397/ liste-des-journaux-electroniques-reconnus-par-la-federation-tunisiennedes-directeurs-de-journaux/

ZBISS Hanene, KHADRAOUI Malek, "Infections nosocomiales : un danger de santé publique mal maîtrisé », Inkyfada, 25 juin 2019. https://inkyfada. $\mathrm{com} / \mathrm{fr} / 2019 / 06 / 25 /$ infections-nosocomiales-un-danger-de-sante-publicmal-maitrise/ 



\section{Les contributeurs}

Maria Adib Doss est attachée temporaire d'enseignement et de recherche (ATER) à l'université de Lille, associée à l'université du Caire et membre du Groupe d'étude et de recherche interdisciplinaire en information et communication (GERiiCO). Elle est également membre associée au Centre d'analyse et de recherche interdisciplinaire sur les médias (CARISM, université Paris 2) et à l'Institut d'études du développement de la Sorbonne (IEDES, université Paris 1).

Chirine Ben Abdallah est chargée d'enseignement à l'université Sorbonne Nouvelle Paris 3, membre associée au Laboratoire communication et politique (LCP)-Irisso (université Paris Dauphine).

Bachir Benaziz est attaché temporaire d'enseignement et de recherche (ATER) à l'université Côte d'Azur, membre de l'URE Transitions (Médias-SavoirTerritoires), ancien post-doctorant à l'ERC Tarica.

Abdelfettah Benchenna est maître de conférences à l'université Sorbonne Paris Nord, membre du Laboratoire des sciences de l'information et de la communication (LabSIC).

Larbi Chouikha est professeur à l'Institut de presse et des sciences de l'information (IPSI) à Tunis.

Cherif Dris est professeur à l'École nationale supérieure de journalisme et des sciences de l'information (ENSJSI) à Alger.

Ahmed Hidass est professeur à l'Institut supérieur de l'information et de la communication (ISIC) à Rabat.

Simon Mangon est doctorant à l'université d'Aix-Marseille, rattaché au laboratoire Mesopolhis (Sciences-Po Aix) et associé à l'Institut français du Proche-Orient (IFPO).

Dominique Marchetti est directeur de recherche au Centre national de la recherche scientifique (CNRS), affecté au Centre européen de sociologie et de science politique (CNRS, EHESS, université Paris 1). 


\title{
Collection Les Rééditions du CJB
}

http://books.openedition.org/cjb/

Naissance du prolétariat marocain : enquête collective, 1948-1950, Robert Montagne, Introduction de la nouvelle édition par Daniel Rivet, 2016 (éd. originale 1951).

Au seuil de la vie marocaine: les coutumes et les relations sociales chez les Marocains, L. Brunot, 2013 (éd. originale 1950).

\section{Collection Description du Maghreb}

\author{
http://books.openedition.org/cjb/
}

Les Parentalités en Afrique musulmane : repenser la famille à partir de l'intérêt de l'enfant et des transformations sociales, sous la direction de Yazid Ben Hounet et Catherine Therrien, 2021.

Réforme et politique dans le monde arabe, Alain Roussillon, 2018.

Fabrique du tourisme et expériences patrimoniales au Maghreb, XIXe-XXIe siècles, sous la direction de Cyril Isnart, Charlotte Mus-Jelidi et Colette Zytnicki, 2018.

Foncier, droit et propriété en Mauritanie: enjeux et perspectives de recherche, sous la direction de Armelle Choplin et Mohamed Fall Ould Bah, 2018.

Les Revendications amazighes dans la tourmente des "Printemps arabes»: trajectoires historiques et évolutions récentes des mouvements identitaires, sous la direction de Mohand Tilmatine et Thierry Desrues, 2017.

Le Droit de la famille au Maroc et son application au sein de la section des affaires familiales du tribunal de première instance de Rabat, sous la direction de Yazid Ben Hounet, Mériam Cheikh, Laïla Benchahda et Nouri Rupert, 2017.

Terrains marocains: sur les traces de chercheurs d'ici et d'ailleurs, sous la direction de Khalid Mouna, Catherine Therrien et Leïla Bouasria, 2017.

La Circulation des productions culturelles: cinémas, informations et séries télévisées dans les mondes arabes et musulmans, Dominique Marchetti (dir.), 2017.

Le Maroc au présent: d'une époque à l'autre, une société en mutation, Baudouin Dupret, Zakaria Rhani, Assia Boutaleb et Jean-Noël Ferrié (dir.), 2016. 


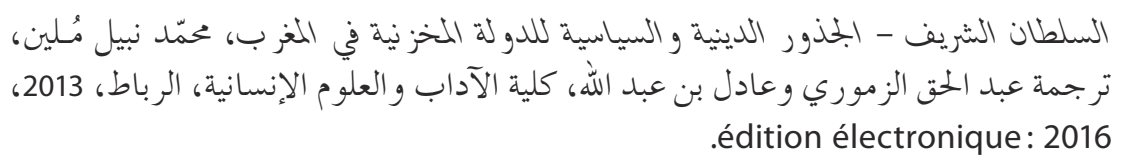

(Nabil Mouline, Le Califat imaginaire d'Ahmad al-Mansûr: pouvoir et diplomatie au Maroc au XVIe siècle, Paris, PUF, 2009).

Migrants au Maroc: cosmopolitisme, présence d'étrangers et transformations sociales, Nadia Khrouz et Nazarena Lanza (dir.), 2016.

Santé, politiques sociales et formes contemporaines de vulnérabilité, sous la direction de Mohammed Ababou et Saâdia Radi, 2015.

L'Esprit du terrain: études anthropologiques au Maroc, Hassan Rachik, 2015.

Des Justices en transition dans le monde arabe? Contributions à une réflexion sur les rapports entre justice et politique, sous la direction d'Éric Gobe, 2015.

Les Ambitions d'une capitale: les projets d'aménagement des fronts d'eau de Rabat, Hicham Mouloudi, 2015.

Fès et sainteté, de la fondation à l'avènement du Protectorat (808-1912): hagiographie, tradition spirituelle et héritage prophétique dans la ville de Mawlāy Idrīs, Ruggero Vimercati Sanseverino, 2014.

Surnaturel et société, l'explication magique de la maladie et du malheur à Khénifra, Maroc, Saâdia Radi, 2013.

Essai sur le système pénal marocain, Mohieddine Amzazi, 2013.

Femmes, associations et politique à Casablanca, Y. Berriane, 2013.

Médinas immuables? Gentrification et changement dans les villes historiques marocaines (1996-2010), sous la direction d'Elsa Coslado, Justin McGuinness et Catherine Miller, 2013.

La Bienvenue et l'adieu: migrants juifs et musulmans au Maghreb, $X V^{e}-X X$ siècles, 3 tomes, sous la direction de Frédéric Abécassis, Karima Dirèche et Rita Aouad, 2012.

Fils de saints contre fils d'esclaves: les pèlerinages de la Zawya d'Imi n'Tatelt (Anti-Atlas et Maroc présaharien), S. Naji, 2011. 
Le Centre Jacques-Berque pour le développement des sciences humaines et sociales au Maroc fait partie du réseau des instituts français de recherche à l'étranger placés sous la co-tutelle du CNRS et du ministère des Affaires étrangères. Sa vocation est de produire un savoir partagé d'excellence en sciences humaines et sociales, de le diffuser, de le valoriser et d'en assurer le renouvellement par des actions de formation. II couvre principalement le territoire du Maroc et celui de la Mauritanie.

Maktabat el-Maghreb, la collection électronique du CJB, accueillie sur le portail OpenEdition, se décline en deux sous-collections: Description $d u$ Maghreb et Les Rééditions du CJB. http://books.openedition.org/cjb/ 

E façon paradoxale, l'étude du journalisme et des médias occupe une place très réduite dans les recherches en sciences sociales et humaines sur l'Afrique du Nord, alors même que les espaces médiatiques dans cette région connaissent depuis les décennies 1990 et 2000 des transformations importantes. Celles-ci se rapportent notamment à l'émergence de nouveaux « acteurs » autres que l'État et les formations politiques autorisées, qui investissent désormais dans ce secteur jugé stratégique : hommes d'affaires, journalistes, communicants, spécialistes du marketing, membres d'organisations internationales, etc. Pour décrire ces bouleversements, les auteurs et autrices de l'ouvrage proposent des enquêtes de terrain en Algérie, en Égypte, au Maroc et en Tunisie, analysant conjointement les transformations des espaces médiatiques, économiques et politiques, ainsi que leurs relations.

En dépit d'une série d'invariants, les trajectoires et les histoires spécifiques de chaque espace national demeurent centrales. La lutte pour la définition des normes professionnelles, les conditions d'exercice du journalisme, la structuration des espaces médiatiques et leurs relations aux champs du pouvoir nationaux varient fortement d'un pays à l'autre. L'ouvrage pointe également la nécessité de comprendre concrètement ce qui est autorisé ou ne l'est pas selon les périodes. L'étude des nouvelles institutions de « régulation » des médias, les conditions d'émergence et de développement de chaînes de télévision, de titres de presse privés non partisans et du déploiement de « programmes d'aide aux médias » sont les trois entrées privilégiées pour saisir les délimitations de l'espace des possibles dans la production et la diffusion de l'offre d'informations.

Bachir Benaziz, enseignant-chercheur contractuel à l'Université Côte d'Azur, membre de l'URE Transitions (Médias-Savoir-Territoires), ancien post-doctorant à l'ERC Tarica.

Abdelfettah Benchenna, enseignant-chercheur à l'Université Sorbonne Paris Nord, membre du Laboratoire des sciences de l'information et de la communication (LabSIC).

Dominique Marchetti, chercheur au Centre national de la recherche scientifique (CNRS), affecté au Centre européen de sociologie et de science politique (CNRS, EHESS, Université Paris 1).

\section{Collection Description du Maghreb}
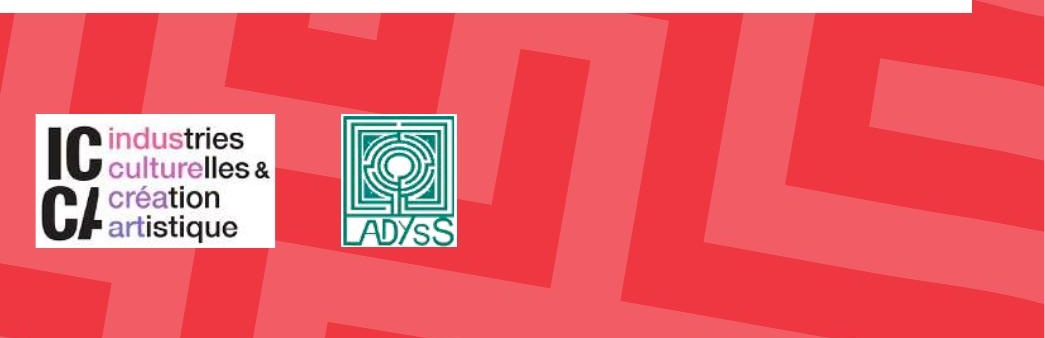